\title{
Increased complexity in interstellar chemistry: detection and chemical modeling of ethyl formate and $n$-propyl cyanide in Sagittarius B2(N) $)^{\star}, \star$
}

\author{
A. Belloche ${ }^{1}$, R. T. Garrod ${ }^{2,1}$, H. S. P. Müller ${ }^{3,1}$, K. M. Menten ${ }^{1}$, C. Comito ${ }^{1}$, and P. Schilke ${ }^{1}$ \\ 1 Max-Planck Institut für Radioastronomie, Auf dem Hügel 69, 53121 Bonn, Germany \\ e-mail: [belloche; kmenten; ccomi to; schilke]@mpifr-bonn.mpg.de \\ 2 Department of Astronomy, Cornell University, 106 Space Sciences Building, Ithaca, NY 14853, USA \\ e-mail: rgarrod@astro.cornell.edu \\ 3 I. Physikalisches Institut, Universität zu Köln, Zülpicher Str. 77, 50937 Köln, Germany \\ e-mail: hspm@ph1.uni-koeln.de
}

Received 19 December 2008 / Accepted 17 February 2009

\begin{abstract}
Context. In recent years, organic molecules of increasing complexity have been found toward the prolific Galactic center source Sagittarius B2.

Aims. We wish to explore the degree of complexity that the interstellar chemistry can reach in star-forming regions.

Methods. We carried out a complete line survey of the hot cores Sgr B2(N) and (M) with the IRAM $30 \mathrm{~m}$ telescope in the $3 \mathrm{~mm}$ range, plus partial surveys at 2 and $1.3 \mathrm{~mm}$. We analyzed this spectral survey in the local thermodynamical equilibrium approximation. We modeled the emission of all known molecules simultaneously, which allows us to search for less abundant, more complex molecules. We compared the derived column densities with the predictions of a coupled gas-phase and grain-surface chemical code.

Results. We report the first detection in space of ethyl formate $\left(\mathrm{C}_{2} \mathrm{H}_{5} \mathrm{OCHO}\right)$ and $n$-propyl cyanide $\left(\mathrm{C}_{3} \mathrm{H}_{7} \mathrm{CN}\right)$ toward $\mathrm{Sgr} \mathrm{B} 2(\mathrm{~N})$. The detection of $n$-propyl cyanide is based on refined spectroscopic parameters derived from combined analyses of available laboratory spectroscopic data. For each molecule, we identified spectral features at the predicted frequencies having intensities compatible with a unique rotation temperature. For an assumed source size of $3^{\prime \prime}$, our modeling yields a column density of $5.4 \times 10^{16} \mathrm{~cm}^{-2}$, a temperature of $100 \mathrm{~K}$, and a linewidth of $7 \mathrm{~km} \mathrm{~s}^{-1}$ for ethyl formate. $n$-Propyl cyanide is detected with two velocity components having column densities of $1.5 \times 10^{16} \mathrm{~cm}^{-2}$ and $6.6 \times 10^{15} \mathrm{~cm}^{-2}$, respectively, for a source size of $3^{\prime \prime}$, a temperature of $150 \mathrm{~K}$, and a linewidth of $7 \mathrm{~km} \mathrm{~s}^{-1}$. The abundances of ethyl formate and $n$-propyl cyanide relative to $\mathrm{H}_{2}$ are estimated to be $3.6 \times 10^{-9}$ and $1.0 \times 10^{-9}$, respectively. We derived column density ratios of $0.8 / 15 / 1$ for the related species $t-\mathrm{HCOOH} / \mathrm{CH}_{3} \mathrm{OCHO} / \mathrm{C}_{2} \mathrm{H}_{5} \mathrm{OCHO}$ and $108 / 80 / 1$ for $\mathrm{CH}_{3} \mathrm{CN} / \mathrm{C}_{2} \mathrm{H}_{5} \mathrm{CN} / \mathrm{C}_{3} \mathrm{H}_{7} \mathrm{CN}$. Our chemical modeling reproduces these ratios reasonably well. It suggests that the sequential, piecewise construction of ethyl and $n$-propyl cyanide from their constituent functional groups on the grain surfaces is their most likely formation route. Ethyl formate is primarily formed on the grains by adding $\mathrm{CH}_{3}$ to functional-group radicals derived from methyl formate, although ethanol may also be a precursor.

Conclusions. The detection in Sgr B2(N) of the next stage of complexity in two classes of complex molecule, esters and alkyl cyanides, suggests that greater complexity in other classes of molecule may be present in the interstellar medium.
\end{abstract}

Key words. astrobiology - astrochemistry - line: identification - stars: formation - ISM: individual objects: Sagittarius B2 -

ISM: molecules

\section{Introduction}

More than 150 molecules have been discovered in the interstellar medium or in circumstellar envelopes over the past four decades (see, e.g., Müller et al. 2005'1). Among them, "complex" organic molecules with up to 13 atoms have been found, showing that the interstellar chemistry in some regions is efficient enough to achieve a relatively high degree of chemical

* Based on observations carried out with the IRAM $30 \mathrm{~m}$ telescope. IRAM is supported by INSU/CNRS (France), MPG (Germany) and IGN (Spain)

$\star \star$ Tables 1, 2, 6, 7, 9, 10, Figs. 1, 3 and Appendix are only available in electronic form at http://www . aanda.org

1 Visit the Cologne Database for Molecular Spectroscopy (CDMS) at http: //www. cdms. de for an updated list. complexity $^{2}$. In addition, much larger molecules have been found in meteorites discovered on Earth, including more than 80 distinct amino acids. The non-terrestrial isotopic ratios of these amino acids, as well as their racemic distributions ${ }^{3}$, suggest that they, or at least their direct precursors, have an interstellar origin (see, e.g., Ehrenfreund et al. 2001; Bernstein et al. 2002; Elsila et al. 2007, and references therein). Interstellar chemistry is therefore very likely capable of producing more complex organic molecules than those discovered in the interstellar medium so far. However, the degree of complexity that

\footnotetext{
2 These molecules are "complex" for astronomers, not for biologists!

3 A racemic distribution means equal amounts of left- and righthanded enantiomers. Enantiomers are stereoisomers that are mirror images of each other and non-superposable.
} 
may be reached is still an open question; the partition functions of larger molecules are large, making it much more difficult to detect such species, even if they are present in reasonably large quantities.

Grain-surface chemistry is frequently invoked as the formation mechanism of many complex species, particularly following recent determinations of some key gas-phase reaction rates. Gasphase production of methyl formate, a molecule ubiquitous in hot-core spectra, appears prohibitively slow (Horn et al. 2004), pointing to an efficient alternative. Additionally, the dissociative recombination of large organic molecular ions with electrons, which is typically the final step in the gas-phase synthesis of complex molecules, appears strongly to favor the fragmentation of complex structure (Geppert et al. 2006).

In the case of hot cores, the granular ice mantles built up during prior phases of evolution present a rich source of simple saturated molecules from which more complex species may form, as has long been realized (Millar et al. 1991). However, while the efficiency of complex molecule formation in the gas phase is limited (not exclusively) by the need to stabilize the energized complex, often resulting in fragmentation, adhesion to a grain surface allows an adduct to quickly thermalize. Thus, molecular radicals derived from the ice mantles may combine in situ on the grain surfaces to build up complex structures efficiently, if dust temperatures are sufficient for the reactants to meet by thermal diffusion. The hot-core models of Garrod \& Herbst (2006) and Garrod et al. (2008) have demonstrated the plausibility of such mechanisms in reproducing observed abundances of many complex organic species.

The detection of new complex molecules places valuable constraints on the chemical models. In the context of the model employed, e.g., by Garrod et al. (2008), obtaining abundances of structurally-related molecules allows one to isolate the chemical behavior of the functional groups from which they are constructed, and to relate these back to more fundamental model parameters such as photodissociation rates, binding energies, and initial ice composition. Such an approach then allows further observational predictions to be made.

One of the current best sources to search for new molecules in the interstellar medium is the hot dense core Sagittarius B2(N) - hereafter Sgr B2(N) for short. This source, dubbed the "Large Molecule Heimat" by Snyder et al. (1994), is extraordinary for its rich molecular content: most complex organic molecules such as, e.g., acetic acid $\left(\mathrm{CH}_{3} \mathrm{COOH}\right.$, Mehringer et al. 1997), glycolaldehyde $\left(\mathrm{CH}_{2}(\mathrm{OH}) \mathrm{CHO}\right.$, Hollis et al. 2000), acetamide ( $\mathrm{CH}_{3} \mathrm{CONH}_{2}$, Hollis et al. 2006), and aminoacetonitrile $\left(\mathrm{NH}_{2} \mathrm{CH}_{2} \mathrm{CN}\right.$, Belloche et al. 2008a,b), were first discovered in Sgr B2(N). This hot core is located in the very massive and extremely active region of high-mass star formation Sagittarius B2, at a projected distance of $\sim 100 \mathrm{pc}$ from the Galactic center, whose distance is $8.0 \pm 0.5 \mathrm{kpc}$ from the Sun (Reid 1993). A second major and somewhat more evolved center of star formation activity, Sgr B2(M), is situated in its vicinity $(\sim 2 \mathrm{pc})$. A more detailed introduction on these two sources and their environment can be found in, e.g., Belloche et al. (2008a).

Here, we report the detection of warm compact emission from ethyl formate $\left(\mathrm{C}_{2} \mathrm{H}_{5} \mathrm{OCHO}\right)$ and $n$-propyl cyanide $\left(\mathrm{C}_{3} \mathrm{H}_{7} \mathrm{CN}\right)$ in $\mathrm{Sgr} \mathrm{B} 2(\mathrm{~N})$ with the IRAM $30 \mathrm{~m}$ telescope. Section 2 summarizes the observational details. The detections of ethyl formate and $n$-propyl cyanide are presented in Sects. 3 and 4, respectively. Implications in terms of interstellar chemistry are discussed in Sect. 5 based on a coupled gas-phase and grain-surface chemical code. Our conclusions are summarized in Sect. 6.

\section{Observations and data analysis}

\subsection{Observations}

We observed the two hot core regions Sgr B2(N) and Sgr B2(M) in January 2004, September 2004, and January 2005 with the IRAM $30 \mathrm{~m}$ telescope on Pico Veleta, Spain. We carried out a complete spectral survey toward both sources in the $3 \mathrm{~mm}$ atmospheric window between 80 and $116 \mathrm{GHz}$. A complete survey was performed in parallel in the $1.3 \mathrm{~mm}$ window between 201.8 and $204.6 \mathrm{GHz}$ and between 205.0 and $217.7 \mathrm{GHz}$. Additional selected spectra were also obtained in the $2 \mathrm{~mm}$ window and between 219 and $268 \mathrm{GHz}$. The coordinates of the observed positions are $\alpha_{\mathrm{J} 2000}=17^{\mathrm{h}} 47^{\mathrm{m}} 20^{\mathrm{s}} .0, \delta_{\mathrm{J} 2000}=-28^{\circ} 22^{\prime} 19.0^{\prime \prime}$ for Sgr B2(N) with a systemic velocity $V_{\mathrm{lsr}}=64 \mathrm{~km} \mathrm{~s}^{-1}$ and $\alpha_{\mathrm{J} 2000}=17^{\mathrm{h}} 47^{\mathrm{m}} 20^{\mathrm{s}} .4, \delta_{\mathrm{J} 2000}=-28^{\circ} 23^{\prime} 07.0^{\prime \prime}$ for Sgr B2(M) with $V_{\mathrm{lsr}}=62 \mathrm{~km} \mathrm{~s}^{-1}$. More details about the observational setup and the data reduction can be found in Belloche et al. (2008a). An rms noise level of $15-20 \mathrm{mK}$ on the $T_{\mathrm{a}}^{\star}$ scale was achieved below $100 \mathrm{GHz}, 20-30 \mathrm{mK}$ between 100 and $114.5 \mathrm{GHz}$, about $50 \mathrm{mK}$ between 114.5 and $116 \mathrm{GHz}$, and $25-60 \mathrm{mK}$ in the $2 \mathrm{~mm}$ window. At $1.3 \mathrm{~mm}$, the confusion limit was reached for most of the spectra obtained toward Sgr B2(N).

\subsection{Modeling of the spectral survey}

The overall goal of our survey was to characterize the molecular content of Sgr B2(N) and (M). It also allows searches for new species once lines emitted by known molecules have been identified, including vibrationally and torsionally excited states, as well as less abundant isotopologues containing, e.g., ${ }^{13} \mathrm{C},{ }^{18} \mathrm{O}$, ${ }^{17} \mathrm{O},{ }^{34} \mathrm{~S},{ }^{33} \mathrm{~S}$, or ${ }^{15} \mathrm{~N}$. We detected about 3700 and 950 lines above $3 \sigma$ over the whole $3 \mathrm{~mm}$ band toward Sgr B2(N) and (M), respectively. These numbers correspond to an average line density of about 100 and 25 features per GHz. Given this high line density, the assignment of a line to a given molecule can be trusted only if all lines emitted by this molecule in our frequency coverage are detected with the right intensity predicted by a model (see below) and no predicted line is missing in the observed spectrum.

We used the XCLASS software (see Comito et al. 2005) to model the emission of all known molecules in the local thermodynamical equilibrium approximation (LTE for short). Each molecule is modeled separately and assumed to be emitted by a uniform region. For each molecule, the free parameters are: source size, temperature, column density, velocity linewidth, velocity offset with respect to the systemic velocity of the source, and a flag indicating if its transitions are in emission or in absorption. For some of the molecules, it was necessary to include several velocity components to reproduce the observed spectra. The velocity components in emission are supposed to be noninteracting, i.e. the intensities add up linearly. This approximation is valid for two distinct, non-overlapping sources smaller than the beam of the telescope, but it is a priori less good for, e.g., a source that consists of a hot, compact region surrounded by a cold, extended envelope or two overlapping sources of spectrally overlapping optically thick emission. More details about the entire analysis are given in Belloche et al. (2008a) and the detailed results of this modeling will be published in a forthcoming article describing the complete survey (Belloche et al., in prep.). So far, we have identified 49 different molecules, 60 rare isotopologues, and lines arising from within 42 vibrationally or torsionally excited states apart from the gound state in Sgr B2(N). This represents about $60 \%$ of the lines detected 
above the $3 \sigma$ level. In Sgr B2(M), the corresponding numbers are $42,53,23$, and $50 \%$, respectively.

\section{Identification of ethyl formate}

\subsection{Ethyl formate frequencies}

Ethyl formate, $\mathrm{C}_{2} \mathrm{H}_{5} \mathrm{OCHO}$, is also known as formic acid ethyl ester, or, according to the International Union of Pure and Applied Chemistry (IUPAC), as ethyl methanoate. Its rotational spectrum was studied in the microwave (Riveros \& Bright Wilson 1967) and in the millimeter wave regions up to $241 \mathrm{GHz}$ (Demaison et al. 1984). The molecule occurs in two conformers. The heavy atoms $\mathrm{C}-\mathrm{C}-\mathrm{O}-\mathrm{C}=\mathrm{O}$ form a planar zigzag chain in the lowest anti-conformer which occasionally is also called the trans-conformer. The two conformers are depicted schematically in Medvedev et al. (2009). The terminal methyl group is rotated by $\sim 95^{\circ}$ to the left or to the right in the gauche-conformer. Because of these two options, the gauche-conformer would be twice as abundant as the anti-conformer if the energy difference between the two were zero. However, the gauche-conformer is $0.78 \pm 0.25 \mathrm{~kJ} \mathrm{~mol}^{-1}$ or $65 \pm 21 \mathrm{~cm}^{-1}$ or $94 \pm 30 \mathrm{~K}$ higher in energy (Riveros \& Bright Wilson 1967). Therefore, the abundance of the gauche-conformer is less than twice that of the anticonformer, in particular at lower temperatures. Since the energy difference has been estimated at room temperature only from relative intensities in the ground state spectra and since excited vibrational states have not been taken into consideration the error in the energy difference may well be larger.

Anti-ethyl formate is a strongly prolate molecule $(A \gg B \approx$ $C$ ) with electric dipole moments for $a$ - and $b$-type transitions, $\mu_{a}$ and $\mu_{b}$, of 1.85 and $0.70 \mathrm{D}$, respectively. The gauche-conformer is more asymmetric, $A$ is smaller by approximately one third and $B$ and $C$ are larger by about one third. The dipole moment components are $\mu_{a}=1.45, \mu_{b}=1.05$, and $\mu_{c}=0.25 \mathrm{D}$ (Riveros $\&$ Bright Wilson 1967). Internal rotation of the terminal methyl group can be neglected. Tunneling between the two gaucheconformers has not been observed (Riveros \& Bright Wilson 1967).

In the early stages of the current study we received additional ethyl formate data from E. Herbst (Medvedev et al. 2009) based on spectra taken at the Ohio State University (OSU) and covering the frequency range 106-378 GHz. The predictions used for the current analysis are based on this data set. An entry for ethyl formate will be available in the catalog section of the Cologne Database for Molecular Spectroscopy (CDMS ${ }^{4}$, see Müller et al. $2001,2005)$. The partition function of ethyl formate is $5.690 \times$ $10^{4}$ and $1.518 \times 10^{4}$ at 150 and $75 \mathrm{~K}$, respectively. In the course of the analysis, the two conformers have been treated separately on occasion to evaluate if the abundance of either conformer is lower than would be expected under LTE conditions.

\subsection{Detection of ethyl formate in Sgr B2(N)}

For us to claim a reliable detection of a new molecule, it is essential that many lines of this molecule be detected in our spectral survey and that all the other expected lines, as predicted by our LTE model, either be blended with lines of other species or be below our detection limit (see Belloche et al. 2008a). Therefore, in the following, we inspect all transitions of ethyl formate in our frequency range. We list in Tables 1 and 2 (online material) only the transitions that our LTE modeling predicts to be

${ }^{4}$ http://www.cdms.de stronger than $20 \mathrm{mK}$ in the main-beam brightness temperature scale. 711 transitions of the anti-conformer and 478 transitions of the gauche-conformer are above this threshold that is conservative since it is below 1.5 times the rms noise level of the best part of our survey (and even below the rms noise level of most parts of our survey). To save some space, when two transitions have a frequency difference smaller than $0.1 \mathrm{MHz}$ that cannot be resolved, we list only the first one. We number the transitions in Col. 1 and give their quantum numbers in Col. 2. The frequencies, the frequency uncertainties, the energies of the lower levels in temperature units, and the $S \mu^{2}$ values are listed in Cols. 3-6, respectively. Since the spectra are in most cases close to the line confusion limit and it is difficult to measure the noise level, we give in Col. 7 the rms sensitivity computed from the system temperature and the integration time: $\sigma=\frac{F_{\mathrm{eff}}}{B_{\mathrm{eff}}} \times \frac{2 T_{\mathrm{sys}}}{\sqrt{\delta f t}}$, with $F_{\mathrm{eff}}$ and $B_{\text {eff }}$ the forward and beam efficiencies, $T_{\text {sys }}$ the system temperature, $\delta f$ the spectral resolution, and $t$ the total integration time (on-source plus off-source).

We list in Col. 8 of Tables 1 and 2 comments about the blends affecting the transitions of the anti- and gauche-conformers of ethyl formate. As can be seen in these tables, most of the ethyl formate lines covered by our survey of Sgr B2(N) are heavily blended with lines of other molecules and therefore cannot be identified in this source based on our single-dish data. Only 46 of the 711 transitions of the anti-conformer are relatively free of contamination from other molecules, known or still unidentified according to our modeling. They are marked "Detected" or "Group detected" in Col. 8 of Table 1, and are listed with more information in Table 3. We stress that all transitions of sufficient strength predicted in the frequency range of our spectral survey are either detected or blended, i.e. no predicted transition is missing in the observed spectrum. The 46 detected transitions correspond to 24 observed features that are shown in Fig. 1 (online material) and labeled in Col. 8 of Table 3. For reference, we show the spectrum observed toward Sgr B2(M) in these figures also. We identified the ethyl formate lines and the blends affecting them with the LTE model of this molecule and the LTE model including all molecules (see Sect. 2.2). The parameters of our best-fit LTE model of ethyl formate are listed in Table 4, and the model is overlaid in red on the spectrum observed toward Sgr B2(N) in Fig. 1. The best-fit LTE model including all molecules is shown in green in the same figures.

For the frequency range corresponding to each detected ethyl formate feature, we list in Table 3 the integrated intensities of the observed spectrum (Col. 10), of the best-fit model of ethyl formate (Col. 11), and of the best-fit model including all molecules (Col. 12). In these columns, the dash symbol indicates transitions belonging to the same feature. Columns 1 to 7 of Table 3 are the same as in Table 1 . The $1 \sigma$ uncertainty given for the integrated intensity in Col. 10 was computed using the estimated noise level of Col. 7.

The measurements of the anti-conformer of ethyl formate are plotted in the form of a population diagram in Fig. 2a, which plots upper level column density divided by statistical weight, $N_{\mathrm{u}} / g_{\mathrm{u}}$, versus the upper level energy in Kelvins (see Goldsmith \& Langer 1999). The data are shown in black and our best-fit model of ethyl formate in red. Out of 12 features encompassing several transitions, one contains transitions with different energy levels and was ignored in the population diagram (feature 17). We used Eq. (A5) of Snyder et al. (2005) to compute the ordinate values:

$$
\ln \left(\frac{N_{\mathrm{u}}}{g_{\mathrm{u}}}\right)=\ln \left(\frac{1.67 W_{T} \times 10^{14}}{S \mu^{2} B v}\right)=-\frac{E_{\mathrm{u}}}{T_{\mathrm{rot}}}+\ln \left(\frac{N_{T}}{Z}\right),
$$


Table 3. Transitions of the anti-conformer of ethyl formate detected toward Sgr B2(N) with the IRAM $30 \mathrm{~m}$ telescope.

\begin{tabular}{|c|c|c|c|c|c|c|c|c|c|c|c|c|}
\hline$N^{a}$ & $\begin{array}{c}\text { Transition } \\
\text { (2) }\end{array}$ & $\begin{array}{l}\text { Frequency } \\
\text { (MHz) } \\
\text { (3) }\end{array}$ & $\begin{array}{l}\text { Unc. }^{b} \\
\text { (kHz) } \\
(4)\end{array}$ & $\begin{array}{l}E_{1}^{c} \\
(\mathrm{~K}) \\
(5)\end{array}$ & $\begin{array}{l}S \mu^{2} \\
\left(D^{2}\right) \\
(6)\end{array}$ & $\begin{array}{c}\sigma^{d} \\
(\mathrm{mK}) \\
(7)\end{array}$ & $F^{e}$ & (9) & $\begin{array}{c}I_{\mathrm{obs}^{g}} \\
\left(\mathrm{~K} \mathrm{~km} \mathrm{~s}^{-1}\right) \\
(10)\end{array}$ & $\begin{array}{l}I_{\text {mod }^{g}} \\
(\mathrm{~K} \mathrm{~km} \\
(11)\end{array}$ & $\begin{array}{c}I_{\text {all }}^{g} \\
\left.\mathrm{~s}^{-1}\right) \\
(12)\end{array}$ & $\begin{array}{c}\text { Comments } \\
\text { (13) }\end{array}$ \\
\hline 1 & $15_{2,14}-14_{2,13}$ & 81779.567 & 4 & 30 & 51 & 13 & 1 & 0.05 & $0.52(06)$ & 0.32 & 0.44 & blend with $U$-line \\
\hline 16 & $15_{6,10}-14_{6,9}$ & 82351.854 & 4 & 54 & 43 & 19 & 2 & 0.07 & $1.02(08)$ & 0.44 & 0.55 & $\begin{array}{l}\text { partial blend with } \mathrm{C}_{2} \mathrm{H}_{5} \mathrm{CN}, \\
v_{13}=1 / v_{21}=1 \text { and } U \text {-line }\end{array}$ \\
\hline 17 & $15_{6,9}-14_{6,8}$ & 82351.858 & 4 & 54 & 43 & 19 & 2 & - & - & - & - & - \\
\hline 26 & $15_{2,13}-14_{2,12}$ & 84081.357 & 4 & 31 & 51 & 19 & 3 & 0.05 & $0.53(08)$ & 0.35 & 0.42 & partial blend with $\mathrm{CH}_{3} \mathrm{CH}_{3} \mathrm{CO}, v_{t}=1$ \\
\hline 28 & $16_{0,16}-15_{0,15}$ & 85065.106 & 4 & 31 & 55 & 22 & 4 & 0.06 & $0.73(10)$ & 0.39 & 0.57 & partial blend with c- $\mathrm{C}_{2} \mathrm{H}_{4} \mathrm{O}$ \\
\hline 44 & $16_{8,8}-15_{8,7}$ & 87810.372 & 4 & 78 & 41 & 17 & 5 & 0.05 & $1.28(07)$ & 0.40 & 1.22 & $\begin{array}{l}\text { partial blend with } \mathrm{CH}_{2}(\mathrm{OH}) \mathrm{CHO} \\
\text { and } \mathrm{C}_{2} \mathrm{H}_{5} \mathrm{CN}\end{array}$ \\
\hline 45 & $16_{8,9}-15_{8,8}$ & 87810.372 & 4 & 78 & 41 & 17 & 5 & - & - & - & - & - \\
\hline 46 & $16_{7,9}-15_{7,8}$ & 87826.665 & 4 & 67 & 44 & 17 & 6 & 0.06 & $0.99(07)$ & 0.48 & 0.86 & partial blend with $\mathrm{HNCO}, v_{5}=1$ \\
\hline 47 & $16_{7,10}-15_{7,9}$ & 87826.665 & 4 & 67 & 44 & 17 & 6 & - & - & - & - & - \\
\hline 53 & $16_{3,14}-15_{3,13}$ & 87993.944 & 4 & 38 & 53 & 19 & 7 & 0.05 & $1.01(08)$ & 0.39 & 0.64 & $\begin{array}{l}\text { partial blend with } \mathrm{CH}_{3} \mathrm{CH}_{3} \mathrm{CO} \\
\text { and } U \text {-line }\end{array}$ \\
\hline 54 & $16_{4,12}-15_{4,11}$ & 88001.562 & 4 & 43 & 51 & 19 & 8 & 0.05 & $0.66(08)$ & 0.35 & 0.62 & blend with $\mathrm{C}_{2} \mathrm{H}_{5} \mathrm{CN}, v_{13}=1 / v_{21}=1$ \\
\hline 73 & $17_{10,7}-16_{10,6}$ & 93284.077 & 4 & 108 & 38 & 22 & 9 & 0.04 & $0.07(09)$ & 0.33 & 0.35 & uncertain baseline \\
\hline 74 & $17_{10,8}-16_{10,7}$ & 93284.077 & 4 & 108 & 38 & 22 & 9 & - & - & - & - & - \\
\hline 75 & $17_{9,8}-16_{9,7}$ & 93292.297 & 4 & 94 & 42 & 22 & 10 & 0.05 & $0.56(09)$ & 0.42 & 0.50 & uncertain baseline \\
\hline 76 & $17_{9,9}-16_{9,8}$ & 93292.297 & 4 & 94 & 42 & 22 & 10 & - & - & - & - & - \\
\hline 77 & $17_{8,9}-16_{8,8}$ & 93304.955 & 4 & 82 & 45 & 22 & 11 & 0.06 & $0.96(09)$ & 0.51 & 0.67 & partial blend with $U$-line \\
\hline 78 & $17_{8,10}-16_{8,9}$ & 93304.955 & 4 & 82 & 45 & 22 & 11 & - & - & - & - & - \\
\hline 79 & $17_{7,11}-16_{7,10}$ & 93324.728 & 4 & 71 & 48 & 22 & 12 & 0.07 & $1.32(09)$ & 0.60 & 0.74 & partial blend with $U$-lines \\
\hline 80 & $17_{7,10}-16_{7,9}$ & 93324.728 & 4 & 71 & 48 & 22 & 12 & - & - & - & - & - \\
\hline 81 & $17_{6,12}-16_{6,11}$ & 93356.821 & 4 & 62 & 51 & 22 & 13 & 0.08 & $1.68(10)$ & 0.70 & 1.24 & blend with $\mathrm{CH}_{3} \mathrm{CH}_{3} \mathrm{CO}$ and $U$-line? \\
\hline 82 & $17_{6,11}-16_{6,10}$ & 93356.838 & 4 & 62 & 51 & 22 & 13 & - & - & - & - & - \\
\hline 83 & $17_{5,13}-16_{5,12}$ & 93412.160 & 4 & 54 & 53 & 22 & 14 & 0.08 & $1.12(10)$ & 0.79 & 0.85 & partial blend with $U$-line? \\
\hline 84 & $17_{5,12}-16_{5,11}$ & 93413.168 & 4 & 54 & 53 & 22 & 14 & - & - & - & - & - \\
\hline 86 & $17_{4,14}-16_{4,13}$ & 93504.972 & 4 & 47 & 55 & 24 & 15 & 0.05 & $1.47(10)$ & 0.44 & 0.87 & blend with $\mathrm{CH}_{3}{ }^{18} \mathrm{OH}$ \\
\hline 87 & $17_{4,13}-16_{4,12}$ & 93539.303 & 4 & 47 & 55 & 24 & 16 & 0.05 & $0.84(10)$ & 0.44 & 0.45 & no blend \\
\hline 94 & $18_{15,3}-17_{15,2}$ & 98760.931 & 5 & 202 & 19 & 18 & 17 & 0.04 & $0.87(09)$ & 0.68 & 0.78 & blend with $U$-line \\
\hline 95 & $18_{15,4}-17_{15,3}$ & 98760.931 & 5 & 202 & 19 & 18 & 17 & - & - & - & - & - \\
\hline 96 & $18_{14,4}-17_{14,3}$ & 98761.079 & 5 & 181 & 24 & 18 & 17 & - & - & - & - & - \\
\hline 97 & $18_{14,5}-17_{14,4}$ & 98761.079 & 5 & 181 & 24 & 18 & 17 & - & - & - & - & - \\
\hline 98 & $18_{16,2}-17_{16,1}$ & 98761.555 & 5 & 224 & 13 & 18 & 17 & - & - & - & - & - \\
\hline 99 & $18_{16,3}-17_{16,2}$ & 98761.555 & 5 & 224 & 13 & 18 & 17 & - & - & - & - & - \\
\hline 100 & $18_{13,5}-17_{13,4}$ & 98762.218 & 5 & 162 & 30 & 18 & 17 & - & - & - & - & - \\
\hline 101 & $18_{13,6}-17_{13,5}$ & 98762.218 & 5 & 162 & 30 & 18 & 17 & - & - & - & - & - \\
\hline 102 & $18_{17,1}-17_{17,0}$ & 98762.790 & 5 & 248 & 7 & 18 & 17 & - & - & - & - & - \\
\hline 103 & $18_{17,2}-17_{17,1}$ & 98762.790 & 5 & 248 & 7 & 18 & 17 & - & - & - & - & - \\
\hline 104 & $18_{12,6}-17_{12,5}$ & 98764.650 & 5 & 144 & 34 & 18 & 17 & - & - & - & - & - \\
\hline 105 & $18_{12,7}-17_{12,6}$ & 98764.650 & 5 & 144 & 34 & 18 & 17 & - & - & - & - & - \\
\hline 118 & $18_{5,14}-17_{5,13}$ & 98928.453 & 4 & 58 & 57 & 18 & 18 & 0.07 & $2.37(09)$ & 0.96 & 2.23 & blend with $\mathrm{C}_{2} \mathrm{H}_{5} \mathrm{CN}, v_{13}=1 / v_{21}=1$ \\
\hline 119 & $18_{5,13}-17_{5,12}$ & 98930.153 & 4 & 58 & 57 & 18 & 18 & - & - & - & - & - \\
\hline 160 & $20_{1,20}-19_{1,19}$ & 105234.713 & 5 & 49 & 68 & 28 & 19 & 0.07 & $1.66(11)$ & 0.76 & 1.27 & blend with $\mathrm{C}_{2} \mathrm{H}_{5} \mathrm{OH}$ and $\mathrm{CH}_{3} \mathrm{OCHO}$ \\
\hline 161 & $19_{1,18}-18_{1,17}$ & 105272.047 & 5 & 47 & 65 & 28 & 20 & 0.07 & $2.07(11)$ & 0.73 & 0.81 & blend with $U$-lines \\
\hline 162 & $199_{3,16}-18_{3,15}$ & 105447.141 & 5 & 52 & 64 & 37 & 21 & 0.06 & $1.07(15)$ & 0.69 & 1.07 & no blend \\
\hline 165 & $20_{2,19}-19_{2,18}$ & 108552.378 & 100 & 53 & 68 & 20 & 22 & 0.07 & $2.05(08)$ & 0.79 & 1.24 & $\begin{array}{l}\text { partial blend with } \mathrm{C}_{2} \mathrm{H}_{3} \mathrm{CN}, v_{15}=1 \\
\text { and } U \text {-lines }\end{array}$ \\
\hline 233 & $21_{6,16}-20_{6,15}$ & 115395.797 & 5 & 81 & 66 & 60 & 23 & 0.11 & $2.94(23)$ & 1.37 & 1.37 & partial blend with $U$-line \\
\hline 234 & $21_{6,15}-20_{6,14}$ & 115395.986 & 5 & 81 & 66 & 60 & 23 & - & - & - & - & - \\
\hline 238 & $22_{1,22}-21_{1,21}$ & 115595.764 & 5 & 59 & 75 & 79 & 24 & 0.08 & $-0.05(30)$ & 0.99 & 2.22 & $\begin{array}{l}\text { blend with } \mathrm{CH}_{3} \mathrm{CH}_{3} \mathrm{CO}, v_{t}=1 \text {, } \\
\text { uncertain baseline }\end{array}$ \\
\hline
\end{tabular}

Notes: ${ }^{a}$ Numbering of the observed transitions associated with a modeled line stronger than $20 \mathrm{mK}$ (see Table 1). ${ }^{b}$ Frequency uncertainty. ${ }^{c}$ Lower energy level in temperature units $\left(E_{\mathrm{l}} / k_{\mathrm{B}}\right) .{ }^{d}$ Calculated rms noise level in $T_{\mathrm{mb}}$ scale. ${ }^{e}$ Numbering of the observed features. ${ }^{f}$ Peak opacity of the modeled feature. ${ }^{g}$ Integrated intensity in $T_{\mathrm{mb}}$ scale for the observed spectrum (Col. 10), the ethyl formate model (Col. 11), and the model including all molecules (Col. 12). The uncertainty in Col. 10 is given in parentheses in units of the last digit.

where $W_{T}$ is the integrated intensity in $\mathrm{K} \mathrm{km} \mathrm{s}^{-1}$ in main-beam brightness temperature scale, $S \mu^{2}$ the line strength times the dipole moment squared in $\mathrm{D}^{2}, B$ the beam filling factor, $v$ the frequency in $\mathrm{GHz}, T_{\text {rot }}$ the rotation temperature in $\mathrm{K}, N_{T}$ the molecular column density in $\mathrm{cm}^{-2}$, and $Z$ the partition function. This equation assumes optically thin emission. To estimate by how much line opacities affect this diagram, we applied the opacity correction factor $C_{\tau}=\frac{\tau}{1-\mathrm{e}^{-\tau}}$ (see Goldsmith \& Langer 1999; Snyder et al. 2005) to the modeled intensities, using the opacities from our radiative transfer calculations (Col. 9 of Table 3); the result is shown in green in Fig. 2a. The population diagram derived from the modeled spectrum is slightly shifted upwards 
Table 4. Parameters of our best-fit LTE model of ethyl formate.

\begin{tabular}{ccccc}
\hline \hline $\begin{array}{c}\mathrm{Size}^{a} \\
\left({ }^{\prime \prime}\right)\end{array}$ & $\begin{array}{c}T_{\text {rot }}{ }^{b} \\
(\mathrm{~K})\end{array}$ & $\begin{array}{c}N^{c} \\
\left(\mathrm{~cm}^{-2}\right)\end{array}$ & $\begin{array}{c}\Delta V^{d} \\
\left(\mathrm{~km} \mathrm{~s}^{-1}\right)\end{array}$ & $\begin{array}{c}V_{\text {off }^{e}} \\
\left(\mathrm{~km} \mathrm{~s}^{-1}\right)\end{array}$ \\
\hline 3.0 & $(2)$ & $(3)$ & $(4)$ & $(5)$ \\
\hline
\end{tabular}

Notes: ${ }^{a}$ Source diameter $(F W H M) .{ }^{b}$ Temperature. ${ }^{c}$ Column density. ${ }^{d}$ Linewidth $(F W H M) .{ }^{e}$ Velocity offset with respect to the systemic velocity of Sgr B2(N) $V_{\mathrm{lsr}}=64 \mathrm{~km} \mathrm{~s}^{-1}$.

but its shape, in particular its slope (the inverse of which approximately determines the rotation temperature), is not significantly changed, since $\ln C_{\tau}$ does not vary much (from 0.019 to 0.053 ). The populations derived from the observed spectrum in the optically thin approximation are therefore not significantly affected by the optical depth of the ethyl formate transitions ${ }^{5}$. The scatter of the black crosses in Fig. 2a is therefore dominated by the blends with other molecules and uncertainties in the baseline removal (indicated by the downwards and upwards blue arrows, respectively).

The population diagram derived from the modeled spectrum in Fig. 2a is systematically below the measurements. Since most of the detected features of the anti-conformer of ethyl formate are partially blended with lines from other molecules (see Col. 13 of Table 3), we can use our model including all identified molecules (shown in green in Fig. 1) to remove the expected contribution from the contaminating molecules. Instead of computing $N_{\mathrm{u}} / g_{\mathrm{u}}$ with the integrated intensities $I_{\mathrm{obs}}$ listed in Col. 10 of Table 3 , we can use the value $I_{\mathrm{obs}}-\left(I_{\mathrm{all}}-I_{\text {mod }}\right)$ derived from Cols. 10-12. The corrected population diagram is shown in Fig. 2b. The predicted (red) and measured (black) points are much closer to each other. A close inspection of Fig. 1 shows however that the wings of most detected features of ethyl formate are still contaminated by $U$-lines, which explains why the measured points are still above the predicted ones in the population diagram (our fitting method with XCLASS is mainly focused on the peak intensity, not on the integrated intensity). The only exception is feature 9 for which the level of the baseline was obviously overestimated (see panel 7 of Fig. 1).

Given the remaining uncertainties due to the contamination from $U$-lines, it is difficult to derive the temperature with high accuracy. However, feature 17, which can unfortunately not be shown in the population diagram since it is a blend of several transitions with different energy levels (from 149 to $253 \mathrm{~K}$ ), is significantly detected in panel 13 of Fig. 1. This is a strong indication that the temperature cannot be much lower than $100 \mathrm{~K}$. Overall, we estimate the resulting uncertainty on the derived column density to be on the order of $25 \%$. Finally, since all detected transitions are optically thin and the region emitting in ethyl formate is most likely compact given its high temperature, column density and source size are degenerate. We fixed the source size to $3^{\prime \prime}$. This is approximately the size of the region emitting in the chemically related molecule methyl formate $\left(\mathrm{CH}_{3} \mathrm{OCHO}\right)$ that we measured with the IRAM Plateau de Bure interferometer (see Table 5 of Belloche et al. 2008b).

From this analysis, we conclude that our best-fit model for the anti-conformer of ethyl formate is fully consistent with our

\footnotetext{
5 Note that our modeled spectrum is anyway calculated with the full LTE radiative transfer that takes into account the optical depth effects (see Sect. 2.2).
}

$30 \mathrm{~m}$ data of Sgr B2(N). This detection of ethyl formate is, to our knowledge, the first one in space ${ }^{6}$.

No feature of the gauche-conformer of ethyl formate is clearly detected in our spectral survey of Sgr B2(N). Only one feature at $213.6 \mathrm{GHz}$ is possibly detected, but the baseline in this frequency range is very uncertain and the feature is blended with a transition of $\mathrm{H}^{13} \mathrm{CCCN}$ (see Table 2). If we consider this feature as a detection, then it implies a column density a factor 2 smaller than for the anti-conformer. This may suggest that the distribution of ethyl formate molecules in the two conformers is not in thermodynamical equilibrium. However, we first have to evaluate the uncertainty on the ratio of the anti- and gauche-conformer populations coming from the uncertainty on the energy difference between the two conformers $(\Delta E=65 \pm$ $21 \mathrm{~cm}^{-1}$, see Sect. 3.1). With $\Delta E=0$, the ratio would be $1 / 2$. For the preferred energy difference of $65 \mathrm{~cm}^{-1}$, we have a ratio of about $0.56 / 0.44$ at $100 \mathrm{~K}$. If we assume an energy difference of $86 \mathrm{~cm}^{-1}$ this ratio would change to $0.62 / 0.38$, i.e. a variation of $\sim 30 \%$. This is not enough to compensate for the factor 2 mentioned above, but can have a significant contribution. In addition, a model of the emission spectrum of the gauche-conformer with the same parameters as for the anti-conformer is not excluded because of the large uncertainty on the baseline at $213.6 \mathrm{GHz}$. Therefore, given the large densities characterizing the hot core in Sgr B2(N) (see, e.g., Belloche et al. 2008a,b), it seems unlikely that the population in the gauche-conformer is subthermal compared to the anti-conformer.

\subsection{Upper limit in Sgr B2(M)}

We do not detect ethyl formate in our spectral survey toward Sgr B2(M). Using the same source size, linewidth, and temperature as for Srg B2(N) (see Table 4), we find $\sim 3 \sigma$ column density upper limits of $2.0 \times 10^{16} \mathrm{~cm}^{-2}$ and $4.0 \times 10^{16} \mathrm{~cm}^{-2}$ in the LTE approximation for the anti- and gauche-conformers, respectively. The column density of ethyl formate is thus at least a factor 3 lower toward Sgr B2(M) than toward Sgr B2(N). This is not surprising since, e.g., Nummelin et al. (2000) found that hot-core-type molecules are more abundant in Sgr B2(N) by factors 3-8 as compared to Sgr B2(M).

6 Jones et al. (2007) tentatively identified three lines detected with the Australia Telescope Compact Array at $~ 86.2738, \sim 86.9784$, and $\sim 86.9787 \mathrm{GHz}$ as two transitions of the anti-conformer of ethyl formate, the second one with two velocity components. However, our model predicts a peak temperature of the ethyl formate transition at $86.977087 \mathrm{GHz}$ on the order of $2 \mathrm{mK}$ whereas the two lines detected with the $30 \mathrm{~m}$ telescope close to this frequency have peak temperatures of 0.38 and $0.65 \mathrm{~K}$, respectively! We identified these two lines with two velocity components of a transition of the vibrationally excited $v_{13}=$ $1 / v_{21}=1$ state of ethyl cyanide, and our modeled spectrum matches the observed lines very well. The tentative identification of Jones et al. (2007) at this frequency is therefore not confirmed. On the other hand, the line detected at $\sim 86.2738 \mathrm{GHz}$ in our survey is still unidentified. The frequency of the $45_{5,41}-44_{6,38}$ transition of ethyl formate mentioned by Jones et al. (2007) comes from the JPL catalog (Pickett et al. 1998, see http://spec.jpl.nasa.gov/). Our catalog contains a significantly different frequency for this transition $(86256.5339 \pm 0.0114 \mathrm{MHz}$ instead of $86273.7945 \pm 0.2103 \mathrm{MHz}$ ), and our model anyway predicts a very low peak temperature on the order of $0.3 \mathrm{mK}$ for this transition. Our catalog contains two other overlapping transitions closer to $86.2738 \mathrm{GHz}\left(35_{10,26}-36_{9,27}\right.$ and $35_{10,25}-36_{9,28}$ at 86.2703101 and $86.2703225 \mathrm{GHz}$, respectively). However, our model predicts a very low peak temperature of $0.6 \mathrm{mK}$ for these transitions as well. Therefore, this tentative identification of Jones et al. (2007) is not confirmed either. 

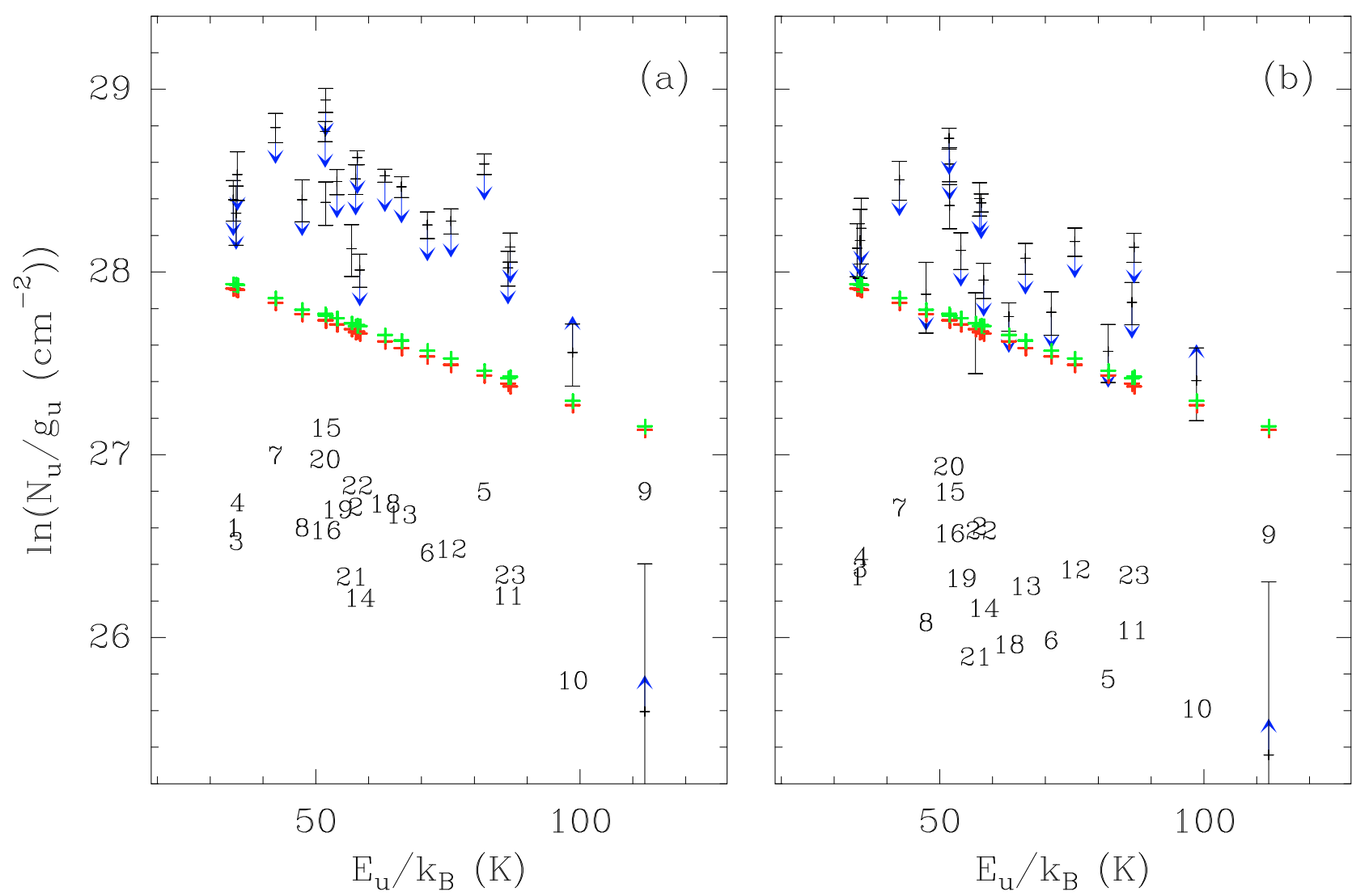

Fig. 2. a) Population diagram of the anti-conformer of ethyl formate in Sgr B2(N). The red points were computed in the optically thin approximation using the integrated intensities of our best-fit model of ethyl formate, while the green points were corrected for the opacity. The black points were computed in the optically thin approximation using the integrated intensities of the spectrum observed with the IRAM $30 \mathrm{~m}$ telescope. The error bars are $1 \sigma$ uncertainties on $N_{\mathrm{u}} / g_{\mathrm{u}}$. Blue arrows pointing downwards mark the transitions blended with transitions from other molecules, while blue arrows pointing upwards indicate that the baseline removed in the observed spectrum is uncertain. The arrow length is arbitrary. The feature labels are shown in black shifted by -1.8 along the Y-axis for clarity, except for feature 9 for which it is shifted by +1.2 . The measurement corresponding to feature 24 (at $E_{\mathrm{u}} / k_{\mathrm{B}}=65 \mathrm{~K}$ ) is not shown since the integrated intensity measured toward Sgr B2(N) is negative, most likely because the level of the baseline was overestimated. Feature 17 is a blend of several transitions with different energy levels and was therefore also omitted. b) Same as a) but with the expected contribution from the contaminating molecules removed from the integrated intensities of the observed spectrum.

\subsection{Comparison to related species}

We easily detect the already known molecules formic acid in the trans form $(t-\mathrm{HCOOH}$ or $t$-HOCHO), methyl formate $\left(\mathrm{CH}_{3} \mathrm{OCHO}\right)$, ethanol $\left(\mathrm{C}_{2} \mathrm{H}_{5} \mathrm{OH}\right)$, and dimethyl ether $\left(\mathrm{CH}_{3} \mathrm{OCH}_{3}\right)$ in our survey toward Sgr B2(N) (see also, e.g., Nummelin et al. 2000; Liu et al. 2001). The parameters of our current best fit models of these molecules are listed in Table 5. All species have two velocity components that correspond to the two hot cores embedded in Sgr B2(N) (see, e.g., Belloche et al. 2008 a, for a discussion about these two sources). Ethyl formate may have a second velocity component too, but our survey is not sensitive enough to detect it with a significant signal-to-noise ratio. Using the same parameters as for the first velocity component but a velocity shift of $10 \mathrm{~km} \mathrm{~s}^{-1}$, we estimate a $3 \sigma$ upper limit of $\sim 2.4 \times 10^{16} \mathrm{~cm}^{-2}$ for the column density of a second velocity component of ethyl formate.

The lines of formic acid are optically thin in our model, so the size of the emitting region cannot be measured with our single-dish data. It was here fixed to $5^{\prime \prime}$, assuming that a more extended region would have a lower temperature. Nummelin et al. (2000) derived a temperature of $74_{-30}^{+82} \mathrm{~K}$ and a beam-averaged column density of $\sim 4.2_{-1.0}^{+2.0} \times 10^{14} \mathrm{~cm}^{-2}$ in the LTE approximation with the SEST telescope $\left(H P B W \sim 23^{\prime \prime}\right.$ at $\left.1.3 \mathrm{~mm}\right)$. They used a linewidth of $13 \mathrm{~km} \mathrm{~s}^{-1}(F W H M)$, which more or less corresponds to the combination of the two velocity components we identified. Their column density translates into a column density of $\sim 9.3_{-2.1}^{+4.4} \times 10^{15} \mathrm{~cm}^{-2}$ for a source size of $5^{\prime \prime}$, i.e. about a factor 2 smaller than the sum of the column densities of both velocity components in Table 5. At least two reasons may explain this discrepancy. First of all, as we noticed in our own partial survey at $1.3 \mathrm{~mm}$, the level of the baseline in this wavelength range is very uncertain for Sgr B2(N) because of the line confusion and it may easily be overestimated. Second, at these high frequencies in Sgr B2(N), the dust is partially optically thick and should partially absorb the line emission ${ }^{7}$. We estimate that the combination of these two effects can lead to underestimating the true line intensities by about a factor 2 or 3 . In addition, assuming a temperature of $200 \mathrm{~K}$, Liu et al. (2001) measured a beam-averaged

${ }^{7}$ Lis et al. (1993) measured a peak flux of $20 \mathrm{Jy} / 4.5^{\prime \prime} \times 3.7^{\prime \prime}$-beam at $227 \mathrm{GHz}$ toward $\mathrm{Sgr} \mathrm{B} 2(\mathrm{~N})$, i.e. $28 \mathrm{~K}$ in temperature unit. For a temperature of $\sim 100 \mathrm{~K}$, this yields a dust optical depth of $\sim 0.34$. On larger scales $\left(\sim 10^{\prime \prime}\right)$, Gordon et al. (1993) estimated that the dust opacity toward Sgr B2(N) reaches a value of 1 at $850 \mu \mathrm{m}$, which implies an opacity of $\sim 0.43-0.53$ at $1.3 \mathrm{~mm}$. As a result, if not taken into account, these significant opacities imply an underestimate of the line intensities by a factor $\sim 1.4-1.7$. 
Table 5. Parameters of our best-fit LTE models of formic acid, methyl formate, ethanol, and dimethyl ether.

\begin{tabular}{|c|c|c|c|c|c|}
\hline $\begin{array}{c}\text { Molecule }^{a} \\
\text { (1) }\end{array}$ & $\begin{array}{c}\text { Size }^{b} \\
\left({ }^{\prime \prime}\right) \\
(2) \\
\end{array}$ & $\begin{array}{c}T_{\text {rot }}{ }^{c} \\
(\mathrm{~K}) \\
(3) \\
\end{array}$ & $\begin{array}{c}N^{d} \\
\left(\mathrm{~cm}^{-2}\right) \\
(4)\end{array}$ & $\begin{array}{c}\Delta V^{e} \\
\left(\mathrm{~km} \mathrm{~s}^{-1}\right) \\
(5)\end{array}$ & $\begin{array}{c}V_{\text {off }}{ }^{\prime} \\
\left(\mathrm{km} \mathrm{s}^{-1}\right) \\
(6) \\
\end{array}$ \\
\hline \multirow[t]{2}{*}{$t$-HCOOH } & 5.0 & 70 & $1.50 \times 10^{16}$ & 8.0 & -1.0 \\
\hline & 5.0 & 70 & $7.50 \times 10^{15}$ & 8.0 & 9.0 \\
\hline \multirow[t]{2}{*}{$\mathrm{CH}_{3} \mathrm{OCHO}$} & 4.0 & 80 & $4.50 \times 10^{17}$ & 7.2 & 0.0 \\
\hline & 4.0 & 80 & $1.50 \times 10^{17}$ & 7.2 & 10.0 \\
\hline \multirow[t]{2}{*}{$\mathrm{C}_{2} \mathrm{H}_{5} \mathrm{OH}$} & 3.0 & 100 & $8.40 \times 10^{17}$ & 8.0 & 0.0 \\
\hline & 3.0 & 100 & $3.00 \times 10^{17}$ & 7.0 & 10.0 \\
\hline \multirow{2}{*}{$\mathrm{CH}_{3} \mathrm{OCH}_{3}$} & 2.5 & 130 & $2.30 \times 10^{18}$ & 6.0 & 0.0 \\
\hline & 2.5 & 130 & $1.10 \times 10^{18}$ & 6.0 & 10.0 \\
\hline
\end{tabular}

Notes: ${ }^{a}$ We used the CDMS entry for $t$ - $\mathrm{HCOOH}$ (version 1 ), and the JPL entries for $\mathrm{CH}_{3} \mathrm{OCHO}$ (ver. 1), $\mathrm{C}_{2} \mathrm{H}_{5} \mathrm{OH}$ (ver. 4), and $\mathrm{CH}_{3} \mathrm{OCH}_{3}$ (ver. 1). See references to the laboratory data therein. ${ }^{b}$ Source diameter $(F W H M) .{ }^{c}$ Temperature. ${ }^{d}$ Column density. ${ }^{e}$ Linewidth $(F W H M)$. $f$ Velocity offset with respect to the systemic velocity of Sgr B2(N) $V_{\mathrm{lsr}}=64 \mathrm{~km} \mathrm{~s}^{-1}$.

column density of $1.1 \pm 0.3 \times 10^{16} \mathrm{~cm}^{-2}$ with the BIMA interferometer at $86-90 \mathrm{GHz}\left(H P B W \sim 14^{\prime \prime} \times 4^{\prime \prime}\right)$. This translates into a column density of $\sim 6.3 \pm 1.5 \times 10^{15} \mathrm{~cm}^{-2}$ for a source size of $5^{\prime \prime}$ and a temperature of $70 \mathrm{~K}$. The interferometric detection of Liu et al. (2001) is somewhat uncertain but suggests that about half of the $30 \mathrm{~m}$ flux may be emitted by an extended region filtered out by the interferometer. The formic acid column density of the compact sources listed in Table 5 may therefore be overestimated by up to a factor 2 .

The lines of methyl formate have opacities of up to about 1 in our model of the $3 \mathrm{~mm}$ spectrum, which puts only weak constraints on the source size that we fixed to 4 ". Assuming a temperature of $200 \mathrm{~K}$, Nummelin et al. (2000) derived a beam-averaged column density of $\sim 5.6_{-0.1}^{+0.3} \times 10^{15} \mathrm{~cm}^{-2}$ with the SEST telescope for the $a$-type lines and, assuming a temperature of $500 \mathrm{~K}, \sim 4.0_{-0.4}^{+0.3} \times 10^{16} \mathrm{~cm}^{-2}$ for the $b$-type lines. For a temperature of $80 \mathrm{~K}$ and a source size of $4^{\prime \prime}$, the column density of the $a$-type lines translates into a column density of $\sim 1.2 \times$ $10^{17} \mathrm{~cm}^{-2}$, which is about a factor 5 smaller than the one we derived here for the sum of the two velocity components. Again, the uncertainty on the level of the baseline and the partial dust absorption at $1.3 \mathrm{~mm}$ may explain part of this discrepancy. In addition, we note that our model at $3 \mathrm{~mm}$ reproduces quite well both the $a$ - and $b$-type lines with the same temperature and column density (see Appendix A, online material), while Nummelin et al. (2000) found an order of magnitude difference between the column densities of the two types. We believe that this discrepancy results from the fact that they did not properly take into account the line blending, which is large in Sgr B2(N) and should affect the (weak) $b$-type lines the most, and that they underestimated the line opacities of the (strong) $a$-type lines that our model predicts to be on the order of $1-3$ in the $1.3 \mathrm{~mm}$ range. Using the BIMA interferometer at $90.15 \mathrm{GHz}$ with a beam size of $14^{\prime \prime} \times 4^{\prime \prime}$, Liu et al. (2001) found a beam-averaged column density of $1.1 \times 10^{17} \mathrm{~cm}^{-2}$ for an assumed temperature of $200 \mathrm{~K}$ in the optically thin approximation. This translates into a column density of $1.5 \times 10^{17} \mathrm{~cm}^{-2}$ for a source size of $4^{\prime \prime}$ and a temperature of $80 \mathrm{~K}$. However, our model predicts an opacity of $\sim 0.6$ for this transition, which implies a higher column density of $2.0 \times$ $10^{17} \mathrm{~cm}^{-2}$. This is still about a factor 2 times lower than our estimate and suggests that, like in the case of formic acid, half of the single-dish flux may actually come from a region more extended than the size of our model and may be filtered out by the interferometer. This conclusion is further supported by the flux ratio of 1.7 between the $12 \mathrm{~m}$ telescope $\left(H P B W=71^{\prime \prime}\right)$ and BIMA $\left(H P B W=25.2^{\prime \prime} \times 6.3^{\prime \prime}\right)$ measurements of Friedel et al. (2004) at $86-90 \mathrm{GHz}$, and the flux ratio of 2.3 we found between the measurements done with the $30 \mathrm{~m}$ telescope and the Plateau de Bure interferometer at $82.2 \mathrm{GHz}$ (see Table 5 of Belloche et al. 2008b). As a result, the methyl formate column density of the compact sources listed in Table 5 may be overestimated by up to a factor 2 .

Most lines of ethanol are optically thin at $3 \mathrm{~mm}(\tau<0.7)$, except for three lines that are marginally optically thick $(\tau \sim$ $1-1.2)$. As a result, the source size is not well contrained and we fixed it to $3^{\prime \prime}$. Nummelin et al. (2000) derived a beam-averaged column density of $4.2 \pm 0.2 \times 10^{15} \mathrm{~cm}^{-2}$ for a temperature of $73_{-4}^{+5} \mathrm{~K}$ with the SEST telescope. This translates into a column density of $2.5 \times 10^{17} \mathrm{~cm}^{-2}$ for a source size of $3^{\prime \prime}$, which is significantly lower than our measurement. However, Nummelin et al. (2000) used an earlier version of the JPL entry for ethanol that turned out to be inaccurate (Pearson, private communication). With this older version, we determined column densities of $2.8 \times 10^{17}$ and $8.9 \times 10^{16} \mathrm{~cm}^{-2}$ for both velocity components, which was consistent with the result of Nummelin et al. (2000). The column densities given in Table 5 were obtained with the latest JPL entry for ethanol (Pearson et al. 2008). The high-energy lines $\left(E_{1} / k_{\mathrm{B}} \sim 40-80 \mathrm{~K}\right)$ detected by Friedel et al. (2004) with the NRAO $12 \mathrm{~m}$ telescope and the BIMA interferometer have the same fluxes with both instruments, implying that they are emitted by a compact region. Only the $4_{1,4}-3_{0.3}$ line with $E_{1} / k_{\mathrm{B}}=5.0 \mathrm{~K}$ has an interferometric flux significantly lower than the single-dish flux. Our LTE model is also too weak for this transition compared to the spectrum obtained with the $30 \mathrm{~m}$ telescope. However, it fits well the low-energy transitions at $84.595868 \mathrm{GHz}\left(E_{1} / k_{\mathrm{B}}=9.4 \mathrm{~K}\right)$ and $112.807174 \mathrm{GHz}$ $\left(E_{1} / k_{\mathrm{B}}=2.1 \mathrm{~K}\right)$ detected in our survey. Therefore, it is unclear whether the BIMA missing flux of the $4_{1,4}-3_{0.3}$ transition suggests an additional cold, extended component, or this line is heavily blended with a transition of another molecule.

Our model of dimethyl ether predicts line opacities up to 2 . The size of the emitting region is thus reasonably well constrained for this molecule. Nummelin et al. (2000) derived a beam-averaged column density of $7.9_{-0.7}^{+0.8} \times 10^{15} \mathrm{~cm}^{-2}$ for a temperature of $197_{-22}^{+31} \mathrm{~K}$ with the SEST telescope. This translates into a column density of $6.8 \times 10^{17} \mathrm{~cm}^{-2}$ for a source size of $2.5^{\prime \prime}$, which is a factor 4 lower than derived here. The discrepancy most likely comes from the beam filling factor of unity assumed by Nummelin et al. (2000) that leads to underestimating the line opacities. Our LTE model indeed predicts line optical depths up to 9 in the $1.3 \mathrm{~mm}$ window.

After rescaling to the same size of $3^{\prime \prime}$, the relative column densities of the three related molecules $t-\mathrm{HCOOH} /$ $\mathrm{CH}_{3} \mathrm{OCHO} / \mathrm{C}_{2} \mathrm{H}_{5} \mathrm{OCHO}$ are about $0.8 / 15 / 1$ for the first velocity component, and $0.9 / 11 / 1$ for the second velocity component using the upper limit found for ethyl formate. We discuss these ratios and the implications for the interstellar chemistry in Sect. 5.

\section{Identification of $n$-propyl cyanide}

\section{1. n-Propyl cyanide frequencies}

$n$-Butanenitrile, $\mathrm{C}_{3} \mathrm{H}_{7} \mathrm{CN}$, is more commonly known as $n$-propyl cyanide or $n$-butyronitrile. Its rotational spectrum has been 
investigated in the microwave (Hirota 1962; Demaison \& Dreizler 1982; Vormann \& Dreizler 1988) and in the millimeter wave regions up to $284 \mathrm{GHz}$ (Włodarczak et al. 1988). The $n$ indicates the normal isomer with the carbon atoms forming a chain, in contrast to the iso isomer which has a branched structure. This isomer has been studied to a lesser extent. However, its rotational spectrum is currently under investigation in Cologne.

$n$-Propyl cyanide exists in two conformers, anti and gauche, just as does ethyl formate. Again, the anti-conformer is the lower energy form, is strongly prolate, and has a large $a$-dipole moment component of $3.60 \mathrm{D}$ and a still sizable $b$-dipole moment component of $0.98 \mathrm{D}$. The gauche-conformer is $1.1 \pm$ $0.3 \mathrm{~kJ} \mathrm{~mol}^{-1}$ or $92 \pm 25 \mathrm{~cm}^{-1}$ or $132 \pm 36 \mathrm{~K}$ higher in energy, more asymmetric, and has $\mu_{a}=3.27$ and $\mu_{b}=2.14 \mathrm{D}$ (Włodarczak et al. 1988). The energy difference has been estimated at room temperature and at $233 \mathrm{~K}$ from relative intensities in the ground state spectra. Since excited vibrational states have not been taken into consideration the error in the energy difference may well be slightly larger than mentioned above. The residuals quoted in the most recent study (Vormann \& Dreizler 1988) for their measurements are frequently much larger than the suggested uncertainties of about $5 \mathrm{kHz}$ suggesting an insufficient set of spectroscopic parameters was used. Moreover, only newly determined rotational and centrifugal distortion parameters were given for the gauche-conformer. Therefore, new sets of rotational and centrifugal distortion parameters were determined for both conformers in the present study.

In the initial fits transition frequencies were taken from all four studies (Hirota 1962; Demaison \& Dreizler 1982; Włodarczak et al. 1988; Vormann \& Dreizler 1988). Two $b$-type transitions from Włodarczak et al. (1988) were omitted from the fits as suggested in the erratum to this paper (Włodarczak et al. 1991). On the other hand, transition frequencies not given in Vormann \& Dreizler (1988), but deposited at the library of the University of Kiel were obtained from there and included in the fits. Uncertainties of 200, 10, 50, and $5 \mathrm{kHz}$ were assigned to the transitions from Hirota (1962), Demaison \& Dreizler (1982), Włodarczak et al. (1988), and Vormann \& Dreizler (1988), respectively. Demaison \& Dreizler (1982) and Vormann \& Dreizler (1988) resolved in part internal rotation of the methyl group or quadrupole splitting of the ${ }^{14} \mathrm{~N}$ nucleus in their laboratory measurements. The methyl internal rotation is unlikely to be resolved in astronomical observations. The quadrupole splitting may be resolvable for some low energy transitions, but these will be generally too weak. Therefore, only the unsplit frequencies were used from these two studies. In the unlikely event of detecting $n$-propyl cyanide in cold sources, quadrupole parameters published in Vormann \& Dreizler (1988) would be adequate.

There were comparatively few transitions reported in Hirota (1962), and their uncertainties were fairly large. Trial fits with these transitions omitted from the fits caused essentially no change in the values and in the uncertainties of the spectroscopic parameters. Therefore, these transitions were omitted from the final fits. Two transitions, $36_{1,36}-35_{0,35}$ of the anticonformer and $31_{5,27}-30_{5,26}$ of the gauche-conformer, had residuals between observed and calculated frequencies larger than four times the experimental uncertainties. Therefore, these transitions were omitted from the data sets. The final line list for the anti-conformer contained 4, 93, and 50 different transition frequencies from Demaison \& Dreizler (1982), Włodarczak et al. (1988), and Vormann \& Dreizler (1988), respectively. The total number of transitions is larger by 62 because of unresolved asymmetry splitting. The corresponding numbers of different transition frequencies for the gauche-conformer are 4 ,
Table 8. Spectroscopic parameters ${ }^{a}(\mathrm{MHz})$ of $n$-propyl cyanide.

\begin{tabular}{lcc}
\hline \hline Parameter & anti & gauche \\
\hline$A$ & $23668.31931(143)$ & $10060.41649(108)$ \\
$B$ & $2268.146892(147)$ & $3267.662408(301)$ \\
$C$ & $2152.963946(168)$ & $2705.459572(290)$ \\
$D_{K} \times 10^{3}$ & $240.653(29)$ & $60.235(6)$ \\
$D_{J K} \times 10^{3}$ & $-10.82631(92)$ & $-18.26470(117)$ \\
$D_{J} \times 10^{6}$ & $398.674(69)$ & $3195.064(207)$ \\
$d_{1} \times 10^{6}$ & $-46.637(42)$ & $-1037.470(55)$ \\
$d_{2} \times 10^{6}$ & $-0.5901(59)$ & $-77.1864(183)$ \\
$H_{K} \times 10^{6}$ & 2.5 & $1.806(18)$ \\
$H_{K J} \times 10^{9}$ & $372.4(24)$ & $-517.3(35)$ \\
$H_{J K} \times 10^{9}$ & $-20.67(20)$ & $9.92(68)$ \\
$H_{J} \times 10^{9}$ & $0.353(11)$ & $4.486(56)$ \\
$h_{1} \times 10^{9}$ & $0.117(14)$ & $2.505(29)$ \\
$h_{2} \times 10^{12}$ & - & $524.6(135)$ \\
$h_{3} \times 10^{12}$ & - & $111.3(31)$ \\
$L_{K K J} \times 10^{12}$ & - & $30.6(31)$ \\
$L_{J K} \times 10^{12}$ & - & $-4.11(78)$ \\
\hline
\end{tabular}

${ }^{a}$ Watson's $S$-reduction was used in the representation $I^{r}$. Numbers in parentheses are one standard deviation in units of the least significant figures. Parameter values with no uncertainties given were estimated. A long dash indicates parameters that are determinable in theory, but could not be determined with significance here.

119, and 46. Unresolved asymmetry splitting causes the total number of transitions to be larger by 46 . The final line lists for both conformers are given in Tables 6 and 7 (online material).

The asymmetry parameter $\kappa=(2 B-A-C) /(A-C)$ is -0.9893 for anti- $n$-propyl cyanide, rather close to the symmetric prolate limit of -1 . In such cases it is advisable to avoid using Watson's $A$-reduction and use the $S$-reduction instead. In the case of the gauche-conformer one finds $\kappa=-0.8471$. In this case both reductions may be used. In the present work the $S$-reduction was used throughout for consistency reason. The sextic distortion parameter $H_{K}$ of the anti-conformer was initially estimated to be smaller than $D_{K}$ by the same factor that that parameter is smaller than $A$. This is certainly only a crude estimate. Trial fits with $H_{K}$ released suggested its value to be slightly larger than this estimate. But since the uncertainty was more than a third of its value and since the difference was smaller than the uncertainty, $H_{K}$ was finally fixed to the estimated value. The final spectroscopic parameters are given in Table 8. Overall, the transition frequencies have been reproduced within experimental uncertainties as the dimensionless rms errors are 0.75 and 0.66 for the anti and gauche-conformers, respectively. The values for the individual data sets do not differ very much from these values. Moreover, this is reasonably close to 1.0 and suggests the ascribed uncertainties are quite appropriate.

The gauche-conformer is considerably more asymmetric than the anti-conformer. Therefore, it is probably not surprising that the distortion parameters describing the asymmetry splitting (the off-diagonal $d_{i}$ and the $h_{i}$ ) are not only larger in magnitude for the former conformer, but also more of these terms are required in the fits. In addition, two octic centrifugal distortion parameters $L$ were needed in the fit of the gauche-conformer resulting in an overall much larger parameter set and thus a much slower converging Hamiltonian compared with the anticonformer. A similar situation occured in the recent investigation of ethyl formate (Medvedev et al. 2009) where also a much larger set of spectroscopic parameters was needed to fit the data of the gauche-conformer compared to the anti-conformer. 
The predictions used for the current analysis will be made available in the CDMS (Müller et al. 2001, 2005, see footnote 4). The partition function of $n$-propyl cyanide is $5.608 \times 10^{4}$ at $150 \mathrm{~K}$. In the course of the analysis, the two conformers again have been treated separately on occasion to evaluate if the abundance of either conformer is lower than would be expected under LTE conditions.

\subsection{Detection of n-propyl cyanide in Sgr B2(N)}

To identify $n$-propyl cyanide, we used the same method as for ethyl formate (see Sect. 3.2). In our spectral survey, 636 transitions of the anti-conformer and 706 transitions of the gaucheconformer are predicted above the threshold of $20 \mathrm{mK}$ defined in Sect. 3.2. They are listed in Tables 9 and 10 (online material), respectively, which are presented in the same way as Tables 1 and 2. Again, as can be seen in these tables, most of the $n$-propyl cyanide lines covered by our survey of Sgr B2(N) are heavily blended with lines of other molecules and therefore cannot be identified in this source. Only 50 of the 636 transitions of the anti-conformer are relatively free of contamination from other molecules, known or still unidentified according to our modeling. They are marked "Detected" or "Group detected" in Col. 8 of Table 9, and are listed with more information in Table 11. We stress that all transitions of sufficient strength predicted in the frequency range of our spectral survey are either detected or blended, i.e. no predicted transition is missing in the observed spectrum. The 50 detected transitions correspond to 12 observed features that are shown in Fig. 3 (online material) and labeled in Col. 8 of Table 11. For reference, we show the spectrum observed toward Sgr B2(M) in these figures also. We identified the $n$-propyl cyanide lines and the blends affecting them with the LTE model of this molecule and the LTE model including all molecules (see Sect. 2.2). The parameters of our best-fit LTE model of $n$-propyl cyanide are listed in Table 12, and the model is overlaid in red on the spectrum observed toward Sgr B2(N) in Fig. 3. The best-fit LTE model including all molecules is shown in green in the same figures.

For the frequency range corresponding to each detected $n$-propyl cyanide feature, we list in Table 11 the integrated intensities of the observed spectrum (Col. 10), of the best-fit model of $n$-propyl cyanide (Col. 11), and of the best-fit model including all molecules (Col. 12). In these columns, the dash symbol indicates transitions belonging to the same feature. Columns 1 to 7 of Table 11 are the same as in Table 9. The $1 \sigma$ uncertainty given for the integrated intensity in Col. 10 was computed using the estimated noise level of Col. 7.

As we did for ethyl formate, we show in Fig. 4a a population diagram derived from the integrated intensities of the detected features of the anti-conformer of $n$-propyl cyanide. Figure $4 \mathrm{~b}$ displays the corresponding diagram after removing the expected contribution from contaminating molecules (see Sect. 3.2 for details). This figure is less helpful than in the case of ethyl formate because all features containing several transitions (6 out of 12) have transitions with different energy levels and cannot be shown in a population diagram. Therefore, this diagram does not help much for the determination of the temperature. Feature 3, which is a blend of transitions with upper energy levels from 61 to $147 \mathrm{~K}$, is however reasonably well fitted by our $150 \mathrm{~K}$ model (see panel 2 of Fig. 3) and gives us some confidence in this high temperature. This is further confirmed by the high temperatures measured in our survey for chemically related molecules (see Sect. 4.4 below).
Our model for the anti-conformer of $n$-propyl cyanide consists of two components with different velocities. The need for a second component mainly comes from the shape of features 2, 9, and 12 . Its velocity is consistent with the velocity of the second component we find for many other, more abundant molecules in our survey toward Sgr B2(N). It was shown interferometrically that this second velocity component is a physically distinct source located $\sim 5^{\prime \prime}$ to the North of the main hot core in Sgr B2(N) (see, e.g., Sect. 3.4 of Belloche et al. 2008a). Our data are consistent with a second component about half as strong in $n$-propyl cyanide as the first component (Table 12). This is also the ratio we found for the two components of ethyl cyanide $\left(\mathrm{C}_{2} \mathrm{H}_{5} \mathrm{CN}\right)$ with the IRAM Plateau de Bure interferometer and the $30 \mathrm{~m}$ telescope (see Table 5 of Belloche et al. 2008b). Finally, since all detected transitions are optically thin and the two regions emitting in $n$-propyl cyanide are most likely compact given their high temperature, column density and source size are degenerated. We fixed the source size to $3^{\prime \prime}$. This is approximately the size of the region emitting in the chemically related molecule ethyl cyanide that we measured with the IRAM Plateau de Bure interferometer (see Table 5 of Belloche et al. 2008b).

From this analysis, we conclude that our best-fit model for the anti-conformer of $n$-propyl cyanide is fully consistent with our $30 \mathrm{~m}$ data of Sgr B2(N). This is, to our knowledge, the first clear detection of this molecule in space ${ }^{8}$.

No feature of the gauche-conformer of $n$-propyl cyanide is clearly detected in our spectral survey of Sgr B2(N). Only one feature at $211.4 \mathrm{GHz}$ is possibly detected but the baseline in this frequency range is very uncertain and this feature is blended with a transition of acetone. If we consider this feature as a detection, it implies a column density a factor 2 smaller than for the model of the anti-conformer, which may suggest a non-thermal distribution of the molecules in the two conformers. However, we first have to evaluate the uncertainty on the ratio of the antiand gauche-conformer populations coming from the uncertainty on their energy difference $\left(\Delta E=92 \pm 25 \mathrm{~cm}^{-1}\right.$, see Sect. 4.1). For $\Delta E=92 \mathrm{~cm}^{-1}$, the anti to gauche ratio is $0.51 / 0.49$ at $150 \mathrm{~K}$, and increases to $0.57 / 0.43$ for $\Delta E=117 \mathrm{~cm}^{-1}$, i.e. a variation of $\sim 30 \%$. This is not enough to explain the factor 2 mentioned above, but it can have a significant contribution. Above all, the uncertainty on the baseline level at $211.4 \mathrm{GHz}$ is quite large and the data are still consistent with a thermal distribution of the gauche- and anti-conformers.

\subsection{Upper limit in Sgr B2(M)}

We do not detect $n$-propyl cyanide in our spectral survey toward Sgr B2(M). Using the same source size, linewidth, and

\footnotetext{
8 Jones et al. (2007) tentatively identified two lines detected with the Australia Telescope Compact Array at $\sim 86.9556$ and $\sim 90.0560 \mathrm{GHz}$ as transitions of the gauche-conformer of $n$-propyl cyanide. However, our model predicts a peak temperature of the $n$-propyl cyanide transition at $86.955466 \mathrm{GHz} 15$ times smaller than the peak temperature $(0.13 \mathrm{~K})$ of the line detected with the $30 \mathrm{~m}$ telescope at this frequency. The tentative identification of Jones et al. (2007) at this frequency is therefore not confirmed. The origin of this line in our survey is still unknown. As far as the other transition is concerned, our model of $n$-propyl cyanide predicts a peak intensity equal to only one quarter of the peak intensity $(0.07 \mathrm{~K})$ of the line detected with the $30 \mathrm{~m}$ telescope at $\sim 90.0560 \mathrm{GHz}$. Since this line is blended with a transition of ${ }^{13} \mathrm{CH}_{3} \mathrm{CH}_{2} \mathrm{CN}$ that has a stronger contribution according to our modeling, the tentative identification of Jones et al. (2007) should be viewed with caution too.
} 
Table 11. Transitions of the anti-conformer of $n$-propyl cyanide detected toward Sgr B2(N) with the IRAM $30 \mathrm{~m}$ telescope.

\begin{tabular}{|c|c|c|c|c|c|c|c|c|c|c|c|c|}
\hline$N^{a}$ & $\begin{array}{c}\text { Transition } \\
\text { (2) }\end{array}$ & $\begin{array}{c}\text { Frequency } \\
(\mathrm{MHz}) \\
(3)\end{array}$ & $\begin{array}{c}\text { Unc. }^{b} \\
(\mathrm{kHz}) \\
(4)\end{array}$ & $\begin{array}{l}E_{1}^{c} \\
(\mathrm{~K}) \\
(5) \\
\end{array}$ & $\begin{array}{l}S \mu^{2} \\
\left(\mathrm{D}^{2}\right) \\
(6) \\
\end{array}$ & $\begin{array}{c}\sigma^{d} \\
(\mathrm{mK}) \\
(7) \\
\end{array}$ & $F^{e}$ & (9) & $\begin{array}{c}I_{\mathrm{obs}}{ }^{g} \\
\left(\mathrm{~K} \mathrm{~km} \mathrm{~s}^{-1}\right) \\
(10)\end{array}$ & $\begin{array}{c}I_{\mathrm{mod}}^{g} \\
(\mathrm{~K} \mathrm{kn} \\
(11) \\
\end{array}$ & $\begin{array}{c}I_{\text {all }^{g}} \\
\left.\mathrm{~s}^{-1}\right) \\
(12)\end{array}$ & $\begin{array}{c}\text { Comments } \\
\text { (13) }\end{array}$ \\
\hline 5 & $19_{6,14}-18_{6,13}$ & 84021.555 & 4 & 73 & 221 & 19 & 1 & 0.06 & $3.27(13)$ & 1.90 & 2.68 & partial blend with $\mathrm{C}_{2} \mathrm{H}_{5} \mathrm{CN}$ \\
\hline 6 & $19_{6,13}-18_{6,12}$ & 84021.556 & 4 & 73 & 221 & 19 & 1 & - & - & - & - & - \\
\hline 7 & $19,12-187,11$ & 84022.819 & 5 & 87 & 212 & 19 & 1 & - & - & - & - & - \\
\hline 8 & $19_{7,13}-18_{7,12}$ & 84022.819 & 5 & 87 & 212 & 19 & 1 & - & - & - & - & - \\
\hline 9 & $19_{5,15}-18_{5,14}$ & 84023.956 & 4 & 62 & 229 & 19 & 1 & - & - & - & - & - \\
\hline 10 & $19_{5,14}-18_{5,13}$ & 84023.960 & 4 & 62 & 229 & 19 & 1 & - & - & - & - & - \\
\hline 11 & $19_{8,11}-18_{8,10}$ & 84026.382 & 5 & 102 & 202 & 19 & 1 & - & - & - & - & - \\
\hline 12 & $19_{8,12}-18_{8,11}$ & 84026.382 & 5 & 102 & 202 & 19 & 1 & - & - & - & - & - \\
\hline 32 & $20_{6,14}-19_{6,13}$ & 88444.212 & 5 & 77 & 235 & 17 & 2 & 0.06 & $4.30(11)$ & 2.31 & 3.28 & $\begin{array}{l}\text { partial blend with } \mathrm{CH}_{3} \mathrm{CH}_{3} \mathrm{CO} \text {, } \\
\mathrm{C}_{2} \mathrm{H}_{5} \mathrm{OH} \text {, and } U \text {-line? }\end{array}$ \\
\hline 33 & $20_{6,15}-19_{6,14}$ & 88444.212 & 5 & 77 & 235 & 17 & 2 & - & - & - & - & - \\
\hline 34 & $20_{7,13}-19_{7,12}$ & 88445.075 & 5 & 91 & 227 & 17 & 2 & - & - & - & - & - \\
\hline 35 & $20_{7,14}-19_{7,13}$ & 88445.075 & 5 & 91 & 227 & 17 & 2 & - & - & - & - & - \\
\hline 36 & $20_{5,16}-19_{5,15}$ & 88447.526 & 5 & 66 & 243 & 17 & 2 & _ & - & - & - & - \\
\hline 37 & $20_{5,15}-19_{5,14}$ & 88447.532 & 5 & 66 & 243 & 17 & 2 & - & - & - & - & - \\
\hline 38 & $20_{8,12}-19_{8,11}$ & 88448.528 & 5 & 106 & 217 & 17 & 2 & - & - & - & - & - \\
\hline 39 & $20_{8,13}-19_{8,12}$ & 88448.528 & 5 & 106 & 217 & 17 & 2 & - & - & - & - & - \\
\hline 40 & $20_{9,11}-19_{9,10}$ & 88453.836 & 5 & 124 & 206 & 17 & 3 & 0.05 & $1.90(11)$ & 1.40 & 2.42 & $\begin{array}{l}\text { partial blend with } \mathrm{C}_{2} \mathrm{H}_{5}{ }^{13} \mathrm{CN} \text {, } \\
{ }^{13} \mathrm{CH}_{3} \mathrm{CH}_{2} \mathrm{CN} \text {, and } \mathrm{CH}_{2}(\mathrm{OH}) \mathrm{CHO}\end{array}$ \\
\hline 41 & $20_{9,12}-19_{9,11}$ & 88453.836 & 5 & 124 & 206 & 17 & 3 & - & - & - & - & - \\
\hline 42 & $20_{4,17}-19_{4,16}$ & 88458.794 & 5 & 57 & 248 & 17 & 3 & - & - & - & - & - \\
\hline 43 & $20_{4,16}-19_{4,15}$ & 88459.387 & 5 & 57 & 248 & 17 & 3 & - & - & - & - & - \\
\hline 44 & $20_{10,10}-19_{10,9}$ & 88460.625 & 5 & 143 & 194 & 17 & 3 & - & - & - & - & - \\
\hline 45 & $20_{10,11}-19_{10,10}$ & 88460.625 & 5 & 143 & 194 & 17 & 3 & - & - & - & - & - \\
\hline 53 & $20_{1,19}-19_{1,18}$ & 89391.938 & 5 & 42 & 258 & 16 & 4 & 0.03 & $0.65(08)$ & 0.44 & 0.62 & blend with $\mathrm{CH}_{3} \mathrm{OCHO}, v_{t}=1$ \\
\hline 55 & $21_{0,21}-20_{0,20}$ & 92164.912 & 5 & 44 & 272 & 27 & 5 & 0.03 & $1.15(14)$ & 0.49 & 0.66 & $\begin{array}{l}\text { blend with } \mathrm{CH}_{3}{ }^{13} \mathrm{CN}, v_{8}=1 \text { and } \\
U \text {-line? }\end{array}$ \\
\hline 57 & $21_{6,16}-20_{6,15}$ & 92866.939 & 5 & 82 & 250 & 28 & 6 & 0.07 & $1.54(21)$ & 3.17 & 3.21 & uncertain baseline \\
\hline 58 & $21_{6,15}-20_{6,14}$ & 92866.939 & 5 & 82 & 250 & 28 & 6 & - & - & - & - & - \\
\hline 59 & $21_{7,14}-20_{7,13}$ & 92867.332 & 5 & 95 & 242 & 28 & 6 & - & - & - & - & - \\
\hline 60 & $21_{7,15}-20_{7,14}$ & 92867.332 & 5 & 95 & 242 & 28 & 6 & - & - & - & - & - \\
\hline 61 & $21_{8,13}-20_{8,12}$ & 92870.627 & 5 & 110 & 232 & 28 & 6 & - & - & - & - & - \\
\hline 62 & $21_{8,14}-20_{8,13}$ & 92870.627 & 5 & 110 & 232 & 28 & 6 & - & - & - & - & - \\
\hline 63 & $21_{5,17}-20_{5,16}$ & 92871.289 & 5 & 70 & 256 & 28 & 6 & - & - & - & - & - \\
\hline 64 & $21_{5,16}-20_{5,15}$ & 92871.298 & 5 & 70 & 256 & 28 & 6 & - & - & - & - & - \\
\hline 65 & $21_{9,12}-20_{9,11}$ & 92875.977 & 5 & 128 & 222 & 28 & 6 & - & - & - & - & - \\
\hline 66 & $21_{9,13}-20_{9,12}$ & 92875.977 & 5 & 128 & 222 & 28 & 6 & - & - & - & - & - \\
\hline 77 & $21_{2,19}-20_{2,18}$ & 93376.934 & 5 & 49 & 269 & 22 & 7 & 0.03 & $0.52(11)$ & 0.49 & 0.51 & no blend \\
\hline 104 & $22_{2,20}-21_{2,19}$ & 97867.394 & 5 & 53 & 282 & 20 & 8 & 0.03 & $0.61(10)$ & 0.58 & 0.63 & uncertain baseline \\
\hline 108 & $10_{4,6}-11_{3,9}$ & 101512.348 & 5 & 23 & 1 & 16 & 9 & 0.03 & $0.67(08)$ & 0.66 & 0.96 & small blend with $\mathrm{CH}_{3} \mathrm{CH}_{3} \mathrm{CO}, v_{t}=1$ \\
\hline 109 & $23_{2,22}-22_{2,21}$ & 101515.201 & 5 & 58 & 295 & 16 & 9 & - & - & - & - & - \\
\hline 110 & $23_{7,16}-22_{7,15}$ & 101711.846 & 5 & 104 & 270 & 16 & 10 & 0.08 & $5.74(11)$ & 4.35 & 6.38 & $\begin{array}{l}\text { partial blend with }{ }^{13} \mathrm{CH}_{2} \mathrm{CHCN} \text {, } \\
\text { uncertain baseline }\end{array}$ \\
\hline 111 & $23_{7,17}-22_{7,16}$ & 101711.846 & 5 & 104 & 270 & 16 & 10 & - & - & - & - & - \\
\hline 112 & $23_{6,18}-22_{6,17}$ & 101712.624 & 5 & 91 & 277 & 16 & 10 & - & - & - & - & - \\
\hline 113 & $23_{6,17}-22_{6,16}$ & 101712.624 & 5 & 91 & 277 & 16 & 10 & - & - & - & - & - \\
\hline 114 & $23_{8,15}-22_{8,14}$ & 101714.680 & 5 & 120 & 262 & 16 & 10 & - & - & - & - & - \\
\hline 115 & $23_{8,16}-22_{8,15}$ & 101714.680 & 5 & 120 & 262 & 16 & 10 & - & - & - & - & - \\
\hline 116 & $23_{5,19}-22_{5,18}$ & 101719.429 & 5 & 79 & 283 & 16 & 10 & - & - & - & - & - \\
\hline 117 & $23_{5,18}-222_{5,17}$ & 101719.450 & 5 & 79 & 283 & 16 & 10 & - & - & - & - & - \\
\hline 118 & $23_{9,14}-22_{9,13}$ & 101720.011 & 5 & 137 & 252 & 16 & 10 & - & - & - & - & - \\
\hline 119 & $23_{9,15}-22_{9,14}$ & 101720.011 & 5 & 137 & 252 & 16 & 10 & - & - & - & - & - \\
\hline 161 & $24_{3,22}-23_{3,21}$ & 106188.197 & 5 & 68 & 306 & 25 & 11 & 0.03 & $0.97(11)$ & 0.76 & 0.82 & noisy \\
\hline 205 & $25_{1,24}-24_{1,23}$ & 111593.662 & 5 & 65 & 323 & 29 & 12 & 0.03 & $1.48(14)$ & 0.90 & 1.08 & no blend \\
\hline
\end{tabular}

Notes: ${ }^{a}$ Numbering of the observed transitions associated with a modeled line stronger than $20 \mathrm{mK}$ (see Table 9). ${ }^{b}$ Frequency uncertainty. ${ }^{c}$ Lower energy level in temperature units $\left(E_{\mathrm{l}} / k_{\mathrm{B}}\right) .{ }^{d}$ Calculated rms noise level in $T_{\mathrm{mb}}$ scale. ${ }^{e}$ Numbering of the observed features. ${ }^{f}$ Peak opacity of the modeled feature. ${ }^{g}$ Integrated intensity in $T_{\mathrm{mb}}$ scale for the observed spectrum (Col. 10), the $n$-propyl cyanide model (Col. 11), and the model including all molecules (Col. 12). The uncertainty in Col. 10 is given in parentheses in units of the last digit.

temperature as for Srg B2(N) (see Table 12), we find a $\sim 3 \sigma$ column density upper limit of $6 \times 10^{15} \mathrm{~cm}^{-2}$ in the LTE approximation for both conformers. The column density of $n$-propyl cyanide is thus at least a factor $\sim 2$ lower toward Sgr B2(M) than toward Sgr B2(N), which is again consistent with the results of, e.g., Nummelin et al. (2000) for other molecules. 

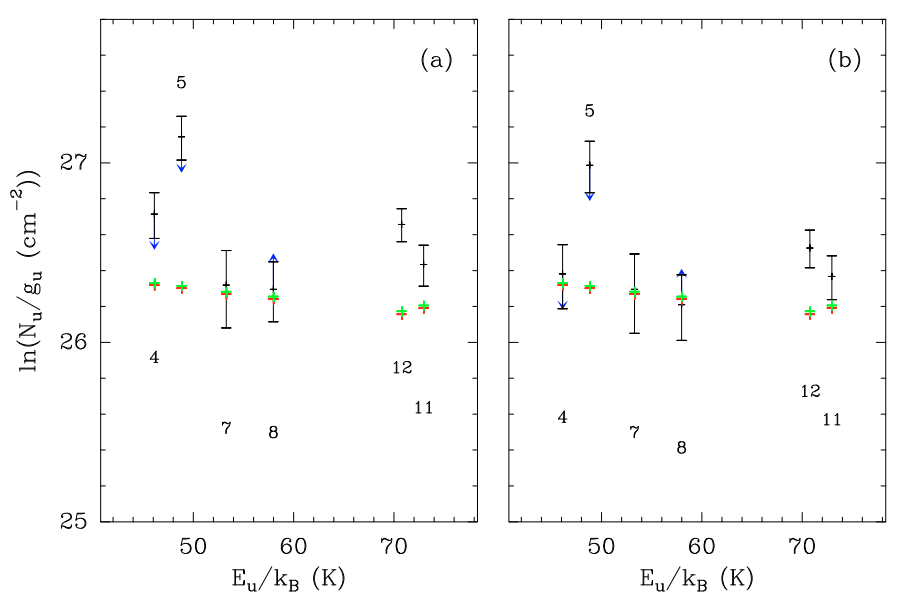

Fig. 4. Population diagram of the anti-conformer of $n$-propyl cyanide presented in the same way as for ethyl formate in Fig. 2 (see the caption of that figure for details). Panel a) shows the population diagram derived from the measured integrated intensities while panel b) presents the population diagram after correction for the expected contribution from contaminating molecules. Features 1, 2, 3, 6, 9, and 10 are blends of several transitions with different energy levels and were therefore omitted.

Table 12. Parameters of our best-fit LTE model of $n$-propyl cyanide with two velocity components.

\begin{tabular}{|c|c|c|c|c|}
\hline $\begin{array}{c}\text { Size }^{a} \\
(") \\
(1) \\
\end{array}$ & $\begin{array}{c}T_{\text {rot }}^{b} \\
(\mathrm{~K}) \\
(2) \\
\end{array}$ & $\begin{array}{c}N^{c} \\
\left(\mathrm{~cm}^{-2}\right) \\
(3)\end{array}$ & $\begin{array}{c}\Delta V^{d} \\
\left(\mathrm{~km} \mathrm{~s}^{-1}\right) \\
(4)\end{array}$ & $\begin{array}{c}V_{\mathrm{off}^{e}} \\
\left(\mathrm{~km} \mathrm{~s}^{-1}\right) \\
(5)\end{array}$ \\
\hline 3.0 & 150 & $1.50 \times 10^{16}$ & 7.0 & -1.0 \\
\hline 3.0 & 150 & $6.60 \times 10^{15}$ & 7.0 & 9.0 \\
\hline
\end{tabular}

Notes: ${ }^{a}$ Source diameter $(F W H M) .{ }^{b}$ Temperature. ${ }^{c}$ Column density. ${ }^{d}$ Linewidth $(F W H M) .{ }^{e}$ Velocity offset with respect to the systemic velocity of Sgr B2(N) $V_{\mathrm{lsr}}=64 \mathrm{~km} \mathrm{~s}^{-1}$.

\subsection{Comparison to related species}

We easily detect the already known molecules methyl cyanide $\left(\mathrm{CH}_{3} \mathrm{CN}\right)$ and ethyl cyanide $\left(\mathrm{C}_{2} \mathrm{H}_{5} \mathrm{CN}\right)$ in our survey toward Sgr B2(N) (see also, e.g., Miao et al. 1995; Liu \& Snyder 1999; Nummelin et al. 2000). The parameters of our current best fit models of these two molecules are listed in Table 13. Our models use also constraints from the weaker isotopologues containing ${ }^{13} \mathrm{C}$ (see, e.g., Müller et al. 2008). The source size is constrained by the optically thick transitions, once the temperature has been fitted. For ethyl cyanide, we used in addition the constraints on the source size derived from our high angular resolution observations with the IRAM Plateau de Bure interferometer (see Table 5 of Belloche et al. 2008b). The first two velocity components detected in methyl cyanide and ethyl cyanide correspond to the two hot cores embedded in Sgr B2(N) (see, e.g., Hollis et al. 2003; Belloche et al. 2008a). They are also seen in $n$-propyl cyanide. In addition, methyl cyanide and ethyl cyanide show a third component that may arise from the blueshifted lobe of an outflow (see the cyanoacetylene $v_{7}=1$ emission in Figs. $5 \mathrm{k}$ to $\mathrm{m}$ of Belloche et al. 2008a). The redshifted counterpart is blended with the northern component in the single-dish beam (see Fig. 3 of Hollis et al. 2003). The third velocity component is too faint to be detected in $n$-propyl cyanide.

The model parameters for the compact sources listed for methyl cyanide in Table 13 are mostly based on the ${ }^{13} \mathrm{C}$ isotopologues with a ${ }^{12} \mathrm{C} /{ }^{13} \mathrm{C}$ isotopic ratio of 20 because the
Table 13. Parameters of our best-fit LTE models of methyl cyanide, ethyl cyanide, vinyl cyanide, and aminoacetonitrile, and column density upper limit for cyanomethylidyne.

\begin{tabular}{|c|c|c|c|c|c|}
\hline $\begin{array}{c}\text { Molecule }^{a} \\
\text { (1) }\end{array}$ & $\begin{array}{c}\text { Size }^{b} \\
(") \\
(2) \\
\end{array}$ & $\begin{array}{c}T_{\text {rot }^{c}} \\
(\mathrm{~K}) \\
(3) \\
\end{array}$ & $\begin{array}{c}N^{d} \\
\left(\mathrm{~cm}^{-2}\right) \\
(4) \\
\end{array}$ & $\begin{array}{c}\Delta V^{e} \\
\left(\mathrm{~km} \mathrm{~s}^{-1}\right) \\
(5) \\
\end{array}$ & $\begin{array}{c}V_{\mathrm{off}^{f}} \\
\left(\mathrm{~km} \mathrm{~s}^{-1}\right) \\
(6) \\
\end{array}$ \\
\hline \multirow[t]{3}{*}{$\mathrm{CH}_{3} \mathrm{CN}$} & 2.7 & 200 & $2.00 \times 10^{18}$ & 6.5 & -1.0 \\
\hline & 2.7 & 200 & $8.00 \times 10^{17}$ & 6.5 & 9.0 \\
\hline & 2.7 & 200 & $1.00 \times 10^{17}$ & 8.0 & -11.0 \\
\hline \multirow[t]{3}{*}{$\mathrm{C}_{2} \mathrm{H}_{5} \mathrm{CN}$} & 3.0 & 170 & $1.20 \times 10^{18}$ & 6.5 & -1.0 \\
\hline & 2.3 & 170 & $1.40 \times 10^{18}$ & 6.5 & 9.0 \\
\hline & 1.7 & 170 & $9.00 \times 10^{17}$ & 8.0 & -11.0 \\
\hline \multirow[t]{3}{*}{$\mathrm{C}_{2} \mathrm{H}_{3} \mathrm{CN}$} & 2.3 & 170 & $8.00 \times 10^{17}$ & 7.0 & -1.0 \\
\hline & 2.3 & 170 & $2.40 \times 10^{17}$ & 7.0 & 9.0 \\
\hline & 2.3 & 170 & $1.00 \times 10^{17}$ & 10.0 & -9.0 \\
\hline $\mathrm{NH}_{2} \mathrm{CH}_{2} \mathrm{CN}$ & 2.0 & 100 & $2.80 \times 10^{16}$ & 7.0 & 0.0 \\
\hline \multirow[t]{2}{*}{$\mathrm{CCN}^{g}$} & 3.0 & 200 & $<1.20 \times 10^{17}$ & 6.5 & -1.0 \\
\hline & 3.0 & 200 & $<1.20 \times 10^{17}$ & 6.5 & 9.0 \\
\hline
\end{tabular}

Notes: ${ }^{a}$ We used the JPL entry for $\mathrm{CH}_{3} \mathrm{CN}$ (version 3), and the CDMS entries for $\mathrm{C}_{2} \mathrm{H}_{5} \mathrm{CN}$ (ver. 1), $\mathrm{C}_{2} \mathrm{H}_{3} \mathrm{CN}$ (ver. 1), $\mathrm{NH}_{2} \mathrm{CH}_{2} \mathrm{CN}$ (ver. 1), and $\mathrm{CCN}$ (ver. 1). See references to the laboratory data therein. ${ }^{b}$ Source diameter (FWHM). ${ }^{c}$ Temperature. ${ }^{d}$ Column density. ${ }^{e}$ Linewidth $(F W H M) .{ }^{f}$ Velocity offset with respect to the systemic velocity of Sgr B2(N) $V_{\mathrm{lsr}}=64 \mathrm{~km} \mathrm{~s}^{-1}$. ${ }^{g}$ The column density upper limit is $\sim 3 \sigma$. The other parameters were fixed.

transitions of the ${ }^{12} \mathrm{C}$ main isotopologue are very optically thick and most likely dominated by large scale emission (see maps of, e.g., de Vicente et al. 1997; Jones et al. 2008). de Vicente et al. (1997) analysed their maps of methyl cyanide emission in the Large Velocity Gradient approximation. They found that the emission consists of several components (hot core, warm envelope, diffuse and hot envelope), and mentioned that their modeling toward Sgr B2(N) is uncertain because of the large opacities. However, their Fig. 5 suggests that the temperature and column density of methyl cyanide are strongly centrally peaked toward Sgr B2(N). Therefore, the emission of the optically thin ${ }^{13} \mathrm{C}$ isotopologues should be dominated by the compact hot cores which gives us some confidence (within a factor of 2) in the column densities listed in Table 13. Friedel et al. (2004) measured similar intensities for $\mathrm{CH}_{3}{ }^{13} \mathrm{CN}$ with the NRAO $12 \mathrm{~m}$ telescope and the BIMA interferometer toward Sgr B2(N), an additional evidence that the compact hot cores dominate the emission of the ${ }^{13} \mathrm{C}$ isotopologues we detected with the $30 \mathrm{~m}$ telescope. For a source size of 2.7", Nummelin et al. (2000) found column densities of $0.7-1.1 \times 10^{17} \mathrm{~cm}^{-2}$ for the ${ }^{13} \mathrm{C}$ isotopologues, which translates into a column density of $1.4-2.2 \times 10^{18} \mathrm{~cm}^{-2}$ for the main isotopologue assuming a ${ }^{12} \mathrm{C} /{ }^{13} \mathrm{C}$ isotopic ratio of 20 . This is in very good agreement with our result (see Table 13).

Assuming a temperature of $200 \mathrm{~K}$ and optically thin emission, Liu et al. (2001) obtained a beam-averaged column density of $4.63 \pm 0.14 \times 10^{16} \mathrm{~cm}^{-2}$ for ethyl cyanide with BIMA at $89.6 \mathrm{GHz}\left(H P B W=14^{\prime \prime} \times 4^{\prime \prime}\right)$. For a source size of $3^{\prime \prime}$, this translates into a column density of $2.9 \times 10^{17} \mathrm{~cm}^{-2}$, which is a factor 4 smaller than the column density we derive for the main velocity component. However, our model predicts peak line opacities of 4-6 for these transitions, which is supported by our simultaneous modeling of the ${ }^{13} \mathrm{C}$ isotopologues of ethyl cyanide (see Müller et al. 2008). As a result, Liu et al. (2001) most likely underestimated the column densities of ethyl cyanide by a factor of a few, which reconciles the single-dish and interferometric measurements and confirms that the source of ethyl cyanide 
emission is compact. This is also confirmed by the reasonable agreement between the $30 \mathrm{~m}$ and Plateau de Bure Interferometer fluxes published by Belloche et al. (2008b) at $81.7 \mathrm{GHz}$ (see their Table 5). The compactness of the source of ethyl cyanide emission most likely explains the discrepancy with the column density found by Nummelin et al. (2000) with SEST in the $1.3 \mathrm{~mm}$ wavelength range $\left(H P B W \sim 23^{\prime \prime}\right)$. These authors derived temperatures of $175_{-20}^{+25} \mathrm{~K}$ and $210_{-30}^{+30} \mathrm{~K}$ and beam-averaged column densities of $1.6_{-0.1}^{+0.2} \times 10^{15} \mathrm{~cm}^{-2}$ and $1.5_{-0.3}^{+0.4} \times 10^{16} \mathrm{~cm}^{-2}$ for the ethyl cyanide $a$ - and $b$-type lines, respectively. While they find an order of magnitude difference between the column densities of the $a$ - and $b$-type lines, we successfully reproduce the ethyl cyanide emission in our $3 \mathrm{~mm}$ survey with a single model for the two types of lines, the former being optically thick while the latter are optically thin. Our model with a small source size predicts line opacities on the order of 10-30 for the $a$-type lines in the $1.3 \mathrm{~mm}$ range. Hence, we believe that the column density derived by Nummelin et al. (2000) for these lines at $1.3 \mathrm{~mm}$ is underestimated by a large factor because they assumed a beam filling factor of 1 , yielding opacities for these lines that were too low. On the other hand, since our model predicts opacities $\lesssim 1$ for the $b$-type lines at $1.3 \mathrm{~mm}$, we would expect the column density derived by these authors to match ours. For a source size of $3^{\prime \prime}$, their column density of the $b$-type lines translates into a column density of $9.0 \times 10^{17} \mathrm{~cm}^{-2}$, which is about a factor 2 smaller than the sum of the column densities of the two main velocity components in Table 13 (after rescaling the second one to a source size of $3^{\prime \prime}$ ). As in Sect. 3.4, we think that the discrepancy arises from the uncertain baseline level and the partial dust absorption in the $1.3 \mathrm{~mm}$ wavelength range. Our current model, which suffers from the same problems, also over-predicts intensities for the lines detected in our partial $1.3 \mathrm{~mm}$ survey.

After rescaling to the same size of $3^{\prime \prime}$, the relative column densities of the three related molecules $\mathrm{CH}_{3} \mathrm{CN} / \mathrm{C}_{2} \mathrm{H}_{5} \mathrm{CN} /$ $\mathrm{C}_{3} \mathrm{H}_{7} \mathrm{CN}$ are $108 / 80 / 1$ for the first velocity component and $98 / 125 / 1$ for the second velocity component. We discuss these ratios and the implications for the interstellar chemistry in Sect. 5.

In addition, we list in Table 13 the best-fit parameters we found for vinyl cyanide (Müller et al. 2008) and aminoacetonitrile (Belloche et al. 2008a), as well as an upper limit for the column density of cyanomethylidyne (CCN) for which the other parameters were fixed.

\section{Chemical modeling and discussion}

To better understand the observational results, we model the chemistry of Sgr B2(N) using a coupled gas-phase and grainsurface chemical code. Garrod et al. (2008) constructed a reaction network to account for the grain-surface formation of many complex molecules observed in hot cores. Surface formation was assumed to occur primarily by the addition of functional-group radicals derived from molecular ices or from other molecules formed in this way. Such reactions are viable when larger radicals become mobile at intermediate grain temperatures $\left(T_{\mathrm{d}} \gtrsim 20 \mathrm{~K}\right)$, achieved during the warm-up to typical hot-core temperatures $(>100 \mathrm{~K})$. The network also includes destruction mechanisms for all complex species, consisting of neutralneutral reactions on the grain surfaces, ion-molecule reactions with simple ions in the gas phase, and cosmic ray-induced photodissociation both in the gas phase and on the grains. To this network we have added appropriate formation and destruction mechanisms for ethyl formate, ethyl and $n$-propyl cyanide, and also the recently identified aminoacetonitrile $\left(\mathrm{NH}_{2} \mathrm{CH}_{2} \mathrm{CN}\right.$,
Belloche et al. 2008a,b), whose surface formation routes may be similar to the other cyanides. In addition, surface hydrogenation routes have been added to allow for the full hydrogenation of the carbon chains $\mathrm{C}_{3}$ and $\mathrm{C}_{4}$, which was not previously considered, as well as the associated hydrogenated species and their destruction channels. The techniques used to construct the new reaction set are presented in detail by Garrod et al. (2008); the current model may be regarded as a consistent extension to that network.

We employ the single-point physical model used by Garrod \& Herbst (2006), in which the isothermal collapse of a diffuse medium, to a density $n_{\mathrm{H}}=10^{7} \mathrm{~cm}^{-3}$, is followed by a warmup from 10 to $200 \mathrm{~K}$. Their $T_{2}$ warm-up profile is assumed, in which the hot-core temperature has a $t^{2}$ dependence on the time, $t$, elapsed in the warm-up phase. Dust and gas temperatures are assumed to be well coupled, hence we let $T=T_{\mathrm{K}}=T_{\text {dust }}$. The warm-up timescale is representative of the time required for a parcel of gas to achieve a temperature of $200 \mathrm{~K}$, as the hot core forms; it therefore does not relate directly to the current infall timescale.

This model traces the evolution of the chemistry up to a temperature of $200 \mathrm{~K}$, associated with the central hot-core region. However, these time-dependent results may also be considered to represent differing spatial extents from the hot-core center, with the innermost regions being the most evolved and achieving the highest temperatures. As such, the time-dependent abundance profiles presented below also indicate a snapshot of the chemistry through the hot core.

Since we are interested mainly in specific features of the model, we choose not to fix the ice composition prior to the warm-up phase, but use the unadulterated composition computed in the collapse-phase.

Other details of the model may be found in Garrod et al. (2008). One important difference is the removal, in keeping with prior chemical networks, of the activation energy barrier for the surface reaction $\mathrm{OH}+\mathrm{H}_{2} \mathrm{CO} \rightarrow \mathrm{HCO}+\mathrm{H}_{2} \mathrm{O}$. Garrod et al. employed an activation energy merely for consistency with other hydrogen-abstraction reactions of $\mathrm{OH}$. The available evidence, however, suggests there is no barrier ${ }^{9}$. This change makes $\mathrm{HCO}$ radicals somewhat more abundant on the grains, tending to increase the final abundances of species such as methyl formate, which is consistent with our observational results.

\subsection{Surface chemistry}

Surface chemical routes for the formation of methyl cyanide, $\mathrm{CH}_{3} \mathrm{CN}$, were already present in the Garrod et al. (2008) network, including direct addition of methyl and nitrile groups, and repetitive surface hydrogenation of gas phase-produced $\mathrm{C}_{2} \mathrm{~N}$. Formation of ethyl cyanide, $\mathrm{C}_{2} \mathrm{H}_{5} \mathrm{CN}$, was limited to repetitive surface hydrogenation of cyanoacetylene $\mathrm{HC}_{3} \mathrm{~N}$ and vinyl cyanide, $\mathrm{C}_{2} \mathrm{H}_{3} \mathrm{CN}$, both of which may be formed in the gas phase. $n$-Propyl cyanide and aminoacetonitrile were not present at all.

Table 14 shows the full set of surface reactions employed in the current model to form methyl cyanide, ethyl cyanide, $n$-propyl cyanide, aminoacetonitrile, and ethylformate, as well as a selection of significant cosmic ray-induced photodissociation processes that may occur on grain surfaces. (The same CR-induced processes are assumed also to occur in the gas

\footnotetext{
9 See the chemical kinetics database of the National Institute of Standards and Technology (NIST), http://kinetics.nist.gov/ kinetics
} 
Table 14. Surface reactions and cosmic-ray induced surface photodissociation processes related to the formation of cyanides, and ethyl formate.

\begin{tabular}{|c|c|c|c|}
\hline Reaction & $\begin{array}{c}\text { Garrod et al. } \\
(2008)\end{array}$ & $\begin{array}{l}\text { Basic } \\
\text { model }\end{array}$ & $\begin{array}{l}\text { Select } \\
\text { model }\end{array}$ \\
\hline $\mathrm{C}_{2} \mathrm{~N}+\mathrm{H} \rightarrow \mathrm{HCCN}$ & $\bullet$ & $\bullet$ & $\bullet$ \\
\hline $\mathrm{HCCN}+\mathrm{H} \rightarrow \mathrm{CH}_{2} \mathrm{CN}$ & $\bullet$ & $\bullet$ & $\bullet$ \\
\hline $\mathrm{CH}_{2} \mathrm{CN}+\mathrm{H} \rightarrow \mathrm{CH}_{3} \mathrm{CN}$ & $\bullet$ & • & $\bullet$ \\
\hline $\mathrm{HC}_{3} \mathrm{~N}+\mathrm{H} \rightarrow \mathrm{C}_{2} \mathrm{H}_{2} \mathrm{CN}(1210 \mathrm{~K})$ & $\bullet$ & $\bullet$ & \\
\hline $\mathrm{C}_{2} \mathrm{H}_{2} \mathrm{CN}+\mathrm{H} \rightarrow \mathrm{C}_{2} \mathrm{H}_{3} \mathrm{CN}$ & • & $\bullet$ & \\
\hline $\mathrm{C}_{2} \mathrm{H}_{3} \mathrm{CN}+\mathrm{H} \rightarrow \mathrm{C}_{2} \mathrm{H}_{4} \mathrm{CN}(750 \mathrm{~K})$ & $\bullet$ & $\bullet$ & \\
\hline $\mathrm{C}_{2} \mathrm{H}_{4} \mathrm{CN}+\mathrm{H} \rightarrow \mathrm{C}_{2} \mathrm{H}_{5} \mathrm{CN}$ & $\bullet$ & $\bullet$ & $\bullet$ \\
\hline $\mathrm{C}_{3} \mathrm{H}_{6} \mathrm{CN}+\mathrm{H} \rightarrow \mathrm{C}_{3} \mathrm{H}_{7} \mathrm{CN}$ & & $\bullet$ & $\bullet$ \\
\hline $\mathrm{CH}_{2}+\mathrm{CN} \rightarrow \mathrm{CH}_{2} \mathrm{CN}$ & $\bullet$ & $\bullet$ & $\bullet$ \\
\hline $\mathrm{CH}_{3}+\mathrm{CN} \rightarrow \mathrm{CH}_{3} \mathrm{CN}$ & $\bullet$ & $\bullet$ & $\bullet$ \\
\hline $\mathrm{CH}_{2}+\mathrm{CH}_{2} \mathrm{CN} \rightarrow \mathrm{C}_{2} \mathrm{H}_{4} \mathrm{CN}$ & & $\bullet$ & $\bullet$ \\
\hline $\mathrm{CH}_{3}+\mathrm{CH}_{2} \mathrm{CN} \rightarrow \mathrm{C}_{2} \mathrm{H}_{5} \mathrm{CN}$ & & $\bullet$ & $\bullet$ \\
\hline $\mathrm{CH}_{2}+\mathrm{C}_{2} \mathrm{H}_{4} \mathrm{CN} \rightarrow \mathrm{C}_{3} \mathrm{H}_{6} \mathrm{CN}$ & & $\bullet$ & $\bullet$ \\
\hline $\mathrm{CH}_{3}+\mathrm{C}_{2} \mathrm{H}_{4} \mathrm{CN} \rightarrow \mathrm{C}_{3} \mathrm{H}_{7} \mathrm{CN}$ & & - & $\bullet$ \\
\hline $\mathrm{C}_{2} \mathrm{H}_{5}+\mathrm{CN} \rightarrow \mathrm{C}_{2} \mathrm{H}_{5} \mathrm{CN}$ & & $\bullet$ & \\
\hline $\mathrm{C}_{3} \mathrm{H}_{7}+\mathrm{CN} \rightarrow \mathrm{C}_{3} \mathrm{H}_{7} \mathrm{CN}$ & & $\bullet$ & \\
\hline $\mathrm{NH}+\mathrm{CH}_{2} \mathrm{CN} \rightarrow \mathrm{NHCH}_{2} \mathrm{CN}$ & & - & $\bullet$ \\
\hline $\mathrm{NH}_{2}+\mathrm{CH}_{2} \mathrm{CN} \rightarrow \mathrm{NH}_{2} \mathrm{CH}_{2} \mathrm{CN}$ & & - & $\bullet$ \\
\hline $\mathrm{H}+\mathrm{NHCH}_{2} \mathrm{CN} \rightarrow \mathrm{NH}_{2} \mathrm{CH}_{2} \mathrm{CN}$ & & $\bullet$ & $\bullet$ \\
\hline $\mathrm{CH}_{2} \mathrm{NH}+\mathrm{CN} \rightarrow \mathrm{NHCH}_{2} \mathrm{CN}$ & & - & \\
\hline $\mathrm{CH}_{2} \mathrm{NH}_{2}+\mathrm{CN} \rightarrow \mathrm{NH}_{2} \mathrm{CH}_{2} \mathrm{CN}$ & & $\bullet$ & \\
\hline $\mathrm{CH}_{3} \mathrm{CN}+h v \rightarrow \mathrm{CH}_{2} \mathrm{CN}+\mathrm{H}$ & & $\bullet$ & $\bullet$ \\
\hline $\mathrm{CH}_{3} \mathrm{CN}+h v \rightarrow \mathrm{CH}_{3}+\mathrm{CN}$ & $\bullet$ & $\bullet$ & $\bullet$ \\
\hline $\mathrm{C}_{2} \mathrm{H}_{5} \mathrm{CN}+h v \rightarrow \mathrm{C}_{2} \mathrm{H}_{4} \mathrm{CN}+\mathrm{H}$ & & $\bullet$ & $\bullet$ \\
\hline $\mathrm{C}_{2} \mathrm{H}_{5} \mathrm{CN}+h v \rightarrow \mathrm{CH}_{3}+\mathrm{CH}_{2} \mathrm{CN}$ & & $\bullet$ & $\bullet$ \\
\hline $\mathrm{C}_{2} \mathrm{H}_{5} \mathrm{CN}+h v \rightarrow \mathrm{C}_{2} \mathrm{H}_{5}+\mathrm{CN}$ & $\bullet$ & $\bullet$ & $\bullet$ \\
\hline $\mathrm{C}_{3} \mathrm{H}_{7} \mathrm{CN}+h v \rightarrow \mathrm{CH}_{3}+\mathrm{C}_{2} \mathrm{H}_{4} \mathrm{CN}$ & & • & $\bullet$ \\
\hline $\mathrm{C}_{3} \mathrm{H}_{7} \mathrm{CN}+h v \rightarrow \mathrm{C}_{2} \mathrm{H}_{5}+\mathrm{CH}_{2} \mathrm{CN}$ & & $\bullet$ & $\bullet$ \\
\hline $\mathrm{C}_{3} \mathrm{H}_{7} \mathrm{CN}+h v \rightarrow \mathrm{C}_{3} \mathrm{H}_{7}+\mathrm{CN}$ & & $\bullet$ & $\bullet$ \\
\hline $\mathrm{NH}_{2} \mathrm{CH}_{2} \mathrm{CN}+h v \rightarrow \mathrm{NH}_{2}+\mathrm{CH}_{2} \mathrm{CN}$ & & $\bullet$ & $\bullet$ \\
\hline $\mathrm{NH}_{2} \mathrm{CH}_{2} \mathrm{CN}+h v \rightarrow \mathrm{NH}_{2} \mathrm{CH}_{2}+\mathrm{CN}$ & & $\bullet$ & $\bullet$ \\
\hline $\mathrm{CH}_{3}+\mathrm{CH}_{2} \mathrm{OCHO} \rightarrow \mathrm{C}_{2} \mathrm{H}_{5} \mathrm{OCHO}$ & & $\bullet$ & $\bullet$ \\
\hline $\mathrm{H}+\mathrm{CH}_{2} \mathrm{OCHO} \rightarrow \mathrm{CH}_{3} \mathrm{OCHO}$ & & $\bullet$ & $\bullet$ \\
\hline $\mathrm{HCO}+\mathrm{C}_{2} \mathrm{H}_{5} \mathrm{O} \rightarrow \mathrm{C}_{2} \mathrm{H}_{5} \mathrm{OCHO}$ & & $\bullet$ & $\bullet$ \\
\hline $\mathrm{H}+\mathrm{C}_{2} \mathrm{H}_{5} \mathrm{O} \rightarrow \mathrm{C}_{2} \mathrm{H}_{5} \mathrm{OH}$ & & $\bullet$ & $\bullet$ \\
\hline $\mathrm{CH}_{3} \mathrm{OCHO}+h v \rightarrow \mathrm{CH}_{2} \mathrm{OCHO}+\mathrm{H}$ & & - & - \\
\hline $\mathrm{C}_{2} \mathrm{H}_{5} \mathrm{OH}+h v \rightarrow \mathrm{C}_{2} \mathrm{H}_{5} \mathrm{O}+\mathrm{H}$ & & $\bullet$ & $\bullet$ \\
\hline $\mathrm{C}_{2} \mathrm{H}_{5} \mathrm{OCHO}+h v \rightarrow \mathrm{CH}_{2} \mathrm{OCHO}+\mathrm{CH}_{3}$ & & $\bullet$ & $\bullet$ \\
\hline $\mathrm{C}_{2} \mathrm{H}_{5} \mathrm{OCHO}+h v \rightarrow \mathrm{C}_{2} \mathrm{H}_{5} \mathrm{O}+\mathrm{HCO}$ & & $\bullet$ & $\bullet$ \\
\hline
\end{tabular}

Notes: Reactions that were present in the hot core model of Garrod et al. (2008) are indicated. Activation energies required for reaction are shown in brackets, where applicable.

phase, at the same rates.) A cosmic-ray ionization rate of $\zeta_{0}=$ $1.3 \times 10^{-17} \mathrm{~s}^{-1}$ is assumed.

The new reactions allow each cyanide to be constructed by sequential formation of its carbon backbone by the addition of $\mathrm{CH}_{2}, \mathrm{CH}_{3}$, or yet larger hydrocarbon radicals; however, photodissociation also allows the break-down of these structures.
The resultant radicals may further react with another functionalgroup radical, to extend the backbone, or with a hydrogen atom, to terminate this sequence. Similarly, aminoacetonitrile may be formed by the addition of $\mathrm{NH}$ or $\mathrm{NH}_{2}$ groups, or by direct addition of $\mathrm{CN}$ to $\mathrm{CH}_{2} \mathrm{NH}_{2}$, or $\mathrm{CH}_{2} \mathrm{NH}$ (followed by hydrogenation). Different routes will dominate according to the relative mobilities of competing radicals, and their availabilities. Hence, the net direction of inter-conversion between cyanides may change with temperature, or as the abundances of molecular precursors vary.

Ethyl formate may be formed on grain surfaces by the addition of a $\mathrm{CH}_{3}$ or $\mathrm{HCO}$ radical to a $\mathrm{CH}_{2} \mathrm{OCHO}$ or $\mathrm{C}_{2} \mathrm{H}_{5} \mathrm{O}$ radical, respectively. These latter species are formed directly by cosmic ray-induced photodissociation of methyl formate or ethanol on the grains; hence, methyl formate need not be the only precursor for ethyl formate, nor the most important one. We do not consider other routes to the formation of $\mathrm{CH}_{2} \mathrm{OCHO}$ and $\mathrm{C}_{2} \mathrm{H}_{5} \mathrm{O}$; radical addition to formaldehyde, $\mathrm{H}_{2} \mathrm{CO}$, would almost certainly be mediated by a substantial activation energy barrier. Alternatively, addition of an oxygen atom to $\mathrm{C}_{2} \mathrm{H}_{5}$ is unlikely to be important, due to the relative scarcity of atomic oxygen, which is mainly bound in the ice mantles as $\mathrm{H}_{2} \mathrm{O}$; however, this route cannot be entirely ruled out.

When the grain surface-produced molecules evaporate, they are subject to gas-phase destruction mechanisms. Whilst cosmic ray-induced photodissociation in the gas phase is also included for consistency, the gas-phase destruction of these molecules is dominated by reaction with the ions $\mathrm{C}^{+}, \mathrm{He}^{+}, \mathrm{H}_{3}{ }^{+}, \mathrm{H}_{3} \mathrm{O}^{+}$and $\mathrm{HCO}^{+}$(followed by dissociative recombination, if a protonated molecule results). Ion-molecule and dissociative recombination reaction rates are of a similar order for all new species; see Garrod et al. (2008).

\subsection{Results}

We analyse the model results for ethyl formate and the cyanides in the context of a selection of complex molecules to which they are chemically or observationally related. We consider first the results of the basic model described above (called hereafter Basic model), using an intermediate warm-up timescale of $2 \times$ $10^{5} \mathrm{yr}$. This timescale was found by Garrod et al. (2008) to be most appropriate to match the abundances of Sgr B2(N).

\subsubsection{Ethyl formate and related species}

Table 15 presents peak fractional abundances, and the temperatures at which they are achieved, derived from the chemical model. Model abundances are converted to values per mean particle with a mean molecular weight, $\mu$, of 2.33 , for comparison to the observations. Also listed are the observed rotational temperatures and abundances (Cols. 7 and 8, respectively). The latter were derived from the column densities given in Tables 4, 5, 12, and 13 , assuming an $\mathrm{H}_{2}$ column density of $1.8 \times 10^{25} \mathrm{~cm}^{-2}$ for a source size of $2^{\prime \prime}$ (see Belloche et al. 2008b), and an $\mathrm{H}_{2}$ column density profile proportional to $r^{-0.5}$ that corresponds to an $\mathrm{H}_{2}$ density profile proportional to $r^{-1.5}$ in spherical symmetry ${ }^{10}$. Given that the dust properties are uncertain by a factor $\sim 2$ at least and that the contribution of the vibrationally or torsionally excited states of some molecules studied here (e.g. ethanol, see Pearson et al. 2008) to their partition function was not included,

10 Osorio et al. (1999) expect a density profile proportional to $r^{-p}$ with
$p=1.5$ for the central region of a hot core. On larger scales in Sgr B2
$\left(20-200^{\prime \prime}\right)$, Lis \& Goldsmith (1989) derived a density profile $p \sim 2-2.5$ while de Vicente et al. (1997) found $p \sim 0.9$. 
Table 15. Peak gas-phase abundances from each model, with corresponding model temperatures, as well as source sizes, rotation temperatures, and gas-phase abundances derived from the observations of the main source in Sgr B2(N).

\begin{tabular}{|c|c|c|c|c|c|c|c|c|}
\hline \multirow[t]{2}{*}{$\overline{\text { Species }}$} & \multicolumn{2}{|c|}{ Basic model } & \multicolumn{2}{|c|}{ Select model } & \multicolumn{3}{|c|}{ Observations } & \multirow{2}{*}{$\begin{array}{l}\text { Abundance ratio } \\
\text { Select model } \\
\text { over observation } \\
\text { (9) }\end{array}$} \\
\hline & $\begin{array}{c}n[i] / n_{\mathrm{H}_{2}} \\
(2)\end{array}$ & $\begin{array}{l}T^{a} \\
(\mathrm{~K}) \\
(3)\end{array}$ & $n[i] / n_{\mathrm{H}_{2}}$ & $\begin{array}{l}T^{a} \\
(\mathrm{~K}) \\
(5)\end{array}$ & $\begin{array}{l}\text { Size } \\
(") \\
(6)\end{array}$ & $\begin{array}{l}T_{\text {rot }} \\
(\mathrm{K}) \\
(7)\end{array}$ & $\begin{array}{c}n[i] / n_{\mathrm{H}_{2}} \\
(8)\end{array}$ & \\
\hline $\mathrm{HCOOH}$ (hot) & $5.4 \mathrm{e}-08$ & 120 & $5.4 \mathrm{e}-08$ & 120 & 5.0 & 70 & $1.3 \mathrm{e}-09$ & 42 \\
\hline HCOOH (cold) & $4.9 \mathrm{e}-09$ & 42 & $4.9 \mathrm{e}-09$ & 42 & 5.0 & 70 & $1.3 e-09$ & 3.8 \\
\hline $\mathrm{CH}_{3} \mathrm{OCHO}$ & $1.7 \mathrm{e}-07$ & 81 & $1.6 \mathrm{e}-07$ & 112 & 4.0 & 80 & $3.5 \mathrm{e}-08$ & 4.6 \\
\hline $\mathrm{C}_{2} \mathrm{H}_{5} \mathrm{OCHO}$ & $2.3 \mathrm{e}-09$ & 110 & $2.3 \mathrm{e}-09$ & 110 & 3.0 & 100 & $3.6 \mathrm{e}-09$ & 0.6 \\
\hline $\mathrm{C}_{2} \mathrm{H}_{5} \mathrm{OH}$ & $1.0 \mathrm{e}-07$ & 120 & $1.0 \mathrm{e}-07$ & 120 & 3.0 & 100 & $5.7 e-08$ & 1.8 \\
\hline $\mathrm{CH}_{3} \mathrm{OCH}_{3}$ & $2.3 e-07$ & 200 & $2.8 \mathrm{e}-07$ & 200 & 2.5 & 130 & $1.4 \mathrm{e}-07$ & 2.0 \\
\hline $\mathrm{CH}_{3} \mathrm{CN}$ & $6.3 e-09$ & 92 & $6.8 \mathrm{e}-09$ & 92 & 2.7 & 200 & $1.3 e-07$ & 0.05 \\
\hline $\mathrm{C}_{2} \mathrm{H}_{5} \mathrm{CN}$ & $4.4 \mathrm{e}-08$ & 117 & $3.3 e-09$ & 117 & 3.0 & 170 & $8.1 \mathrm{e}-08$ & 0.04 \\
\hline $\mathrm{C}_{3} \mathrm{H}_{7} \mathrm{CN}$ & $3.5 \mathrm{e}-08$ & 120 & $4.0 \mathrm{e}-11$ & 123 & 3.0 & 150 & $1.0 \mathrm{e}-09$ & 0.04 \\
\hline $\mathrm{NH}_{2} \mathrm{CH}_{2} \mathrm{CN}$ & $1.4 \mathrm{e}-08$ & 117 & $1.2 \mathrm{e}-08$ & 117 & 2.0 & 100 & $1.5 \mathrm{e}-09$ & 8.0 \\
\hline $\mathrm{C}_{2} \mathrm{H}_{3} \mathrm{CN}$ & $1.2 \mathrm{e}-08$ & 200 & $5.1 \mathrm{e}-08$ & 92 & 2.3 & 170 & $4.7 e-08$ & 1.1 \\
\hline
\end{tabular}

Notes: ${ }^{a}$ The model temperatures are the temperatures at which the peak gas-phase abundances are achieved.

we estimate these observed abundances to be accurate within a factor $\sim 3$.

Ethyl formate is clearly formed most significantly at late times (see Fig. 5a), and its grain-surface abundance (dotted red lines) scales well with that of methyl formate. Grain-surface methyl formate is, in fact, the primary source of precursor radicals (via photodissociation) for the formation of ethyl formate. When methyl formate evaporates, and ethanol is left as the dominant source of precursor radicals, ethyl formate production becomes dependent on the addition of $\mathrm{HCO}$ to $\mathrm{C}_{2} \mathrm{H}_{5} \mathrm{O}$. The postevaporation gas-phase abundance of ethyl formate relative to methyl formate and formic acid appears to match observational abundances and rotational temperatures reasonably well.

The gas-phase methyl formate peak abundance is also relatively close to the observed abundance (within a factor 5), and the model temperature at this peak is in very good agreement with the observed rotational temperature (see Table 5). However, the abundance quickly falls, and the ratio of gas phase $\mathrm{CH}_{3} \mathrm{OCHO}$ to $\mathrm{HCOOH}, \mathrm{C}_{2} \mathrm{H}_{5} \mathrm{OH}$ and $\mathrm{CH}_{3} \mathrm{OCH}_{3}$ at the higher temperatures most appropriate to the densest regions of the hot core is low compared to the observed values.

The Basic model uses the same binding energies for methyl formate and dimethyl ether as were employed by Garrod et al. (2008), appropriate to binding on amorphous water ice. These values cause relatively early evaporation of those species, resulting in significant destruction in the gas-phase, and low fractional abundances in comparison to observed values in the case of methyl formate. The binding energies of those molecules were obtained by simple interpolation of measured values obtained for other species. Laboratory data for methyl formate and dimethyl ether evaporation from appropriate ice surfaces are not currently available.

For species comprising at least one - $\mathrm{OH}$ functional group, binding-energy estimates take account of hydrogen-bonding interactions with the ice surface. Such species may act as both hydrogen-bond donors and acceptors, raising their binding strengths. However, both methyl formate and dimethyl ether have at least one unbonded electron pair attached to a strongly electro-negative atom (oxygen), allowing them to be hydrogenbond acceptors. This may give them a somewhat stronger bond to the predominantly water-ice surface than has been assumed.

Here, the binding energy of methyl formate is raised beyond that of the Basic model, such that it falls approximately half way between its old value and that of ethanol, its most closely-matched counterpart with a single, fully hydrogenbonding functional group. This augmentation constitutes an increase of approximately $1000 \mathrm{~K}$, giving $E_{D}=5200 \mathrm{~K}$. The binding energy of dimethyl ether is similarly raised by $1000 \mathrm{~K}$.

Augmentation of methyl formate binding energy allows it to remain on grains for longer, reducing the time available for gas-phase destruction, before the majority of other species evaporate, damping the effect of ion-molecule destruction pathways (see Fig. 5b). This allows gas-phase methyl formate fractional abundances to remain high for longer, although the resulting peak-abundance temperature is somewhat greater, at $112 \mathrm{~K}$.

Dimethyl ether does have a viable gas-phase formation mechanism, and is largely produced in the gas phase, due to the large abundance of methanol $\left(\sim 10^{-5} n_{\mathrm{H}}\right)$; hence, the peak abundance is not strongly affected by the augmentation of its binding energy. Its gas-phase abundance in the model is consistent with the observed value (within a factor 2 , see Table 15). The peak-abundance temperature of the model is somewhat higher than that derived observationally. A slightly lower grain-surface methanol abundance would remedy this, as post-evaporation gas-phase methanol abundances should diminish more rapidly, reducing the rate of dimethyl ether formation. A slower warmup subsequent to methanol evaporation would also produce a similar effect. Nevertheless, the observed rotational temperature of dimethyl ether seems consistent with gas-phase formation.

Surface formation rates of ethyl formate, methyl formate and ethanol are not strictly dependent on methanol abundance in the ices, but rather on the rate of formation of its photodissociation products, $\mathrm{CH}_{3} \mathrm{O}, \mathrm{CH}_{2} \mathrm{OH}$, and $\mathrm{CH}_{3}$. These rates are not well constrained; however, they seem appropriate for this model. A lower grain-surface methanol abundance, as suggested above, would therefore necessitate slightly greater methanol photodissociation rates, in order to achieve appropriate abundances for methyl formate and other surface-formed species. Gas-phase and grain-surface ethyl formate abundances are largely unaffected by the changes in methyl formate binding energy. Both the gasphase and grain-surface abundances of formic acid are strongly dependent on gas-phase processes (see Garrod et al. 2008). As a result, there appears to be no simple correlation with ethyl or methyl formate abundances. However, the low rotational temperature reported in Sect. 3.4 is qualitatively consistent with the low-temperature gas-phase formic acid peak at 40-60 K, a point noted by Garrod et al. (2008) in comparison to other hot-core observations. This "cold" peak presents a fractional abundance 

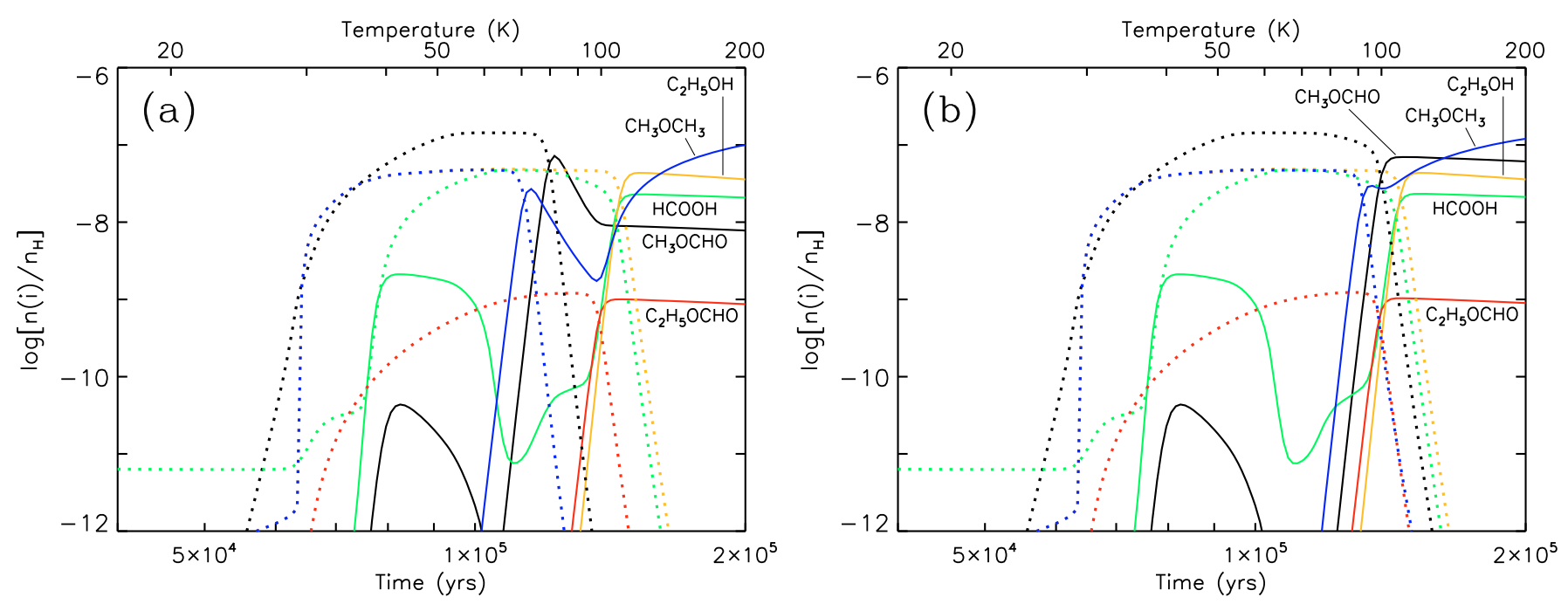

Fig. 5. a) Basic model, showing methyl formate, ethyl formate, formic acid, and related species. b) The same species, following augmentation of methyl formate and dimethyl ether binding energies. Solid lines indicate gas-phase species; dotted lines of the same color indicate the same species on the grain surfaces.
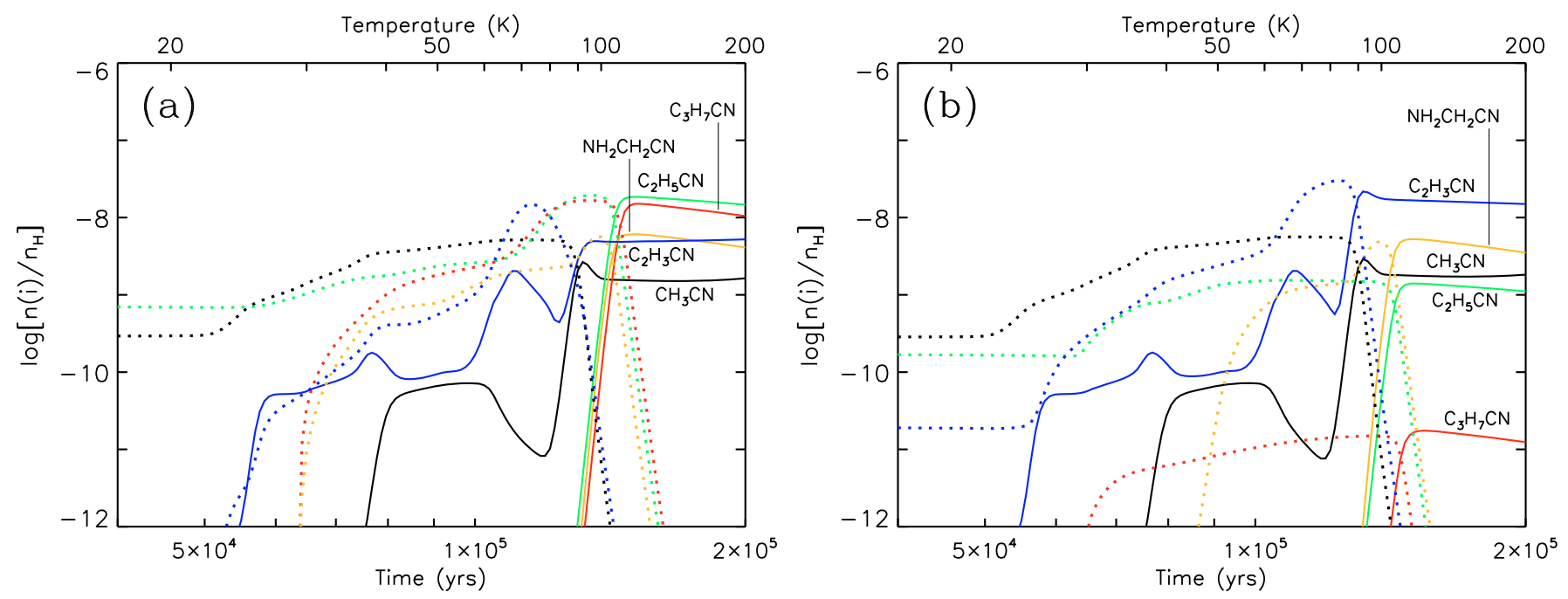

Fig. 6. a) Basic model, showing cyanides. b) The same species, using the Select model, in which selected grain-surface reactions are de-activated (see Table 14). Solid lines indicate gas-phase species; dotted lines of the same color indicate the same species on the grain surfaces.

very close to the observed value (within a factor 4 , see Table 15 ). In Sect 3.4, we modeled the spectrum of formic acid using a single temperature component; however, a two-component model with rotational temperatures (and inferred spatial extents) appropriate to the chemical models is not noticeably worse than the single-component fit. As discussed in Sect. 3.4, the existence of both hot, compact and cold, extended components would be consistent with the lower flux measured with the BIMA interferometer by Liu et al. (2001) compared to our lower-resolution single-dish measurement.

\subsubsection{Cyanides}

The Basic model is capable of producing cyanide species in appropriate absolute quantities (see Fig. 6a), however, their relative abundances are not well matched to the observationally determined values. In order to understand the behavior of the cyanide network, the different grain-surface formation mechanisms, and combinations, were isolated by artificially de-activating particular reaction routes. In fact, all combinations that include either the hydrogenation of the cyanopolyyne $\mathrm{HC}_{3} \mathrm{~N}$ and of vinyl cyanide, $\mathrm{C}_{2} \mathrm{H}_{3} \mathrm{CN}$, or the addition of large, pre-formed hydrocarbons directly to the $\mathrm{CN}$ radical, produce wildly inaccurate ratios. In some such cases, $n$-propyl cyanide is the most abundant of all, often with methyl cyanide abundances deeply depressed. The only combination in which the correct proportion is reproduced is that in which only the sequential addition of grain-surface $\mathrm{CH}_{2}$ and $\mathrm{CH}_{3}$ functional groups is allowed (see Fig. 6b). We label this model, combined with the augmented binding energies of methyl formate and dimethyl ether, as the Select model. In this scheme, formation of the larger cyanides begins with cosmic ray-induced photodissociation of a smaller grain-surface alkyl cyanide molecule (resulting in the ejection of a hydrogen atom), or with the accretion of $\mathrm{CH}_{2} \mathrm{CN}$ (which may 
be formed in the gas-phase following the evaporation of HCN). A methyl-group radical is then added to produce a larger alkyl cyanide molecule.

Methyl cyanide itself is mainly formed on the grains by addition of $\mathrm{CH}_{3}$ and $\mathrm{CN}$ radicals, but it may also be formed by gas-phase processes fuelled by the evaporation of HCN. Methyl cyanide evaporates from the dust grains around $90 \mathrm{~K}$, producing its greatest gas-phase abundance; however, the subsequent evaporation of all molecular material from the grains promotes rapid gas-phase formation, maintaining methyl cyanide abundances for longer, and providing qualitative agreement with the large rotational temperature derived from the observational data.

The abundance obtained for aminoacetonitrile is in reasonable agreement with that obtained observationally (within a factor 8), suggesting that the addition of $\mathrm{NH}$ or $\mathrm{NH}_{2}$ to $\mathrm{CH}_{2} \mathrm{CN}$ on grain surfaces, similar to the suggested mechanism for ethyl cyanide, is a plausible route to its formation. There may therefore be some degree of correlation between these two species, which should be investigated in future. The removal of the other formation routes for aminoacetonitrile, comprising the addition of grain-surface $\mathrm{CN}$ to either $\mathrm{CH}_{2} \mathrm{NH}$ or $\mathrm{CH}_{2} \mathrm{NH}_{2}$, makes little difference to the results, mainly due to limited availability of the latter two radicals.

Vinyl cyanide, $\mathrm{C}_{2} \mathrm{H}_{3} \mathrm{CN}$, a potential precursor of ethyl cyanide and $n$-propyl cyanide, is formed predominantly in the gas-phase in both the Basic and Select models. This occurs through the reaction of $\mathrm{CN}$ with ethylene $\left(\mathrm{C}_{2} \mathrm{H}_{4}\right)$, which has been shown experimentally to be rapid over a range of temperatures (Carty et al. 2001). The resultant gas-phase vinyl cyanide then accretes onto the grains until greater temperatures are achieved. Following evaporation of the ice mantles at $T>$ $100 \mathrm{~K}$, vinyl cyanide is again formed rapidly in the gas-phase by the same mechanism, allowing it, like methyl cyanide, to retain large fractional abundances longer than the other cyanides. This effect is also in qualititative agreement with its relatively high rotational temperature. Both models show good agreement with the observational abundance of this molecule, but the Select model produces an excellent match (see Table 15).

For the Basic model, ratios of peak abundance values are $\mathrm{HCOOH} / \mathrm{CH}_{3} \mathrm{OCHO} / \mathrm{C}_{2} \mathrm{H}_{5} \mathrm{OCHO}=23 / 72 / 1$ and $\mathrm{CH}_{3} \mathrm{CN} /$ $\mathrm{C}_{2} \mathrm{H}_{5} \mathrm{CN} / \mathrm{C}_{3} \mathrm{H}_{7} \mathrm{CN}=0.18 / 1.3 / 1$. For the Select model, these ratios are $\mathrm{HCOOH} / \mathrm{CH}_{3} \mathrm{OCHO} / \mathrm{C}_{2} \mathrm{H}_{5} \mathrm{OCHO}=23 / 70 / 1$ and $\mathrm{CH}_{3} \mathrm{CN} / \mathrm{C}_{2} \mathrm{H}_{5} \mathrm{CN} / \mathrm{C}_{3} \mathrm{H}_{7} \mathrm{CN}=171 / 82 / 1$. These seem a fair match to the observed values of Sects. 3.4 and $4.4(0.8 / 15 / 1$ and $108 / 80 / 1$, respectively). Consideration of only the low temperature formic acid peak in the models further improves its ratio with ethyl formate abundances.

The warm-up timescale of $t_{\max }=2 \times 10^{5} \mathrm{yr}$ appears to yield the most appropriate reproduction of observed cyanide ratios, although longer timescales are also plausible; the Select model, with $t_{\max }=10^{6} \mathrm{yr}$, produces peak abundance ratios of $\mathrm{HCOOH} / \mathrm{CH}_{3} \mathrm{OCHO} / \mathrm{C}_{2} \mathrm{H}_{5} \mathrm{OCHO}=4.2 / 3.3 / 1$ and $\mathrm{CH}_{3} \mathrm{CN} /$ $\mathrm{C}_{2} \mathrm{H}_{5} \mathrm{CN} / \mathrm{C}_{3} \mathrm{H}_{7} \mathrm{CN}=258 / 106 / 1$.

\subsection{Discussion}

Based on the abundance ratios of the model, the dominant formation mechanism for alkyl cyanides is probably the sequential addition of $\mathrm{CH}_{2}$ or $\mathrm{CH}_{3}$ radicals to $\mathrm{CN}, \mathrm{CH}_{2} \mathrm{CN}$ and $\mathrm{C}_{2} \mathrm{H}_{4} \mathrm{CN}$ on the grain surfaces. Both the alternative routes - the grain-surface hydrogenation of gas phase-formed $\mathrm{HC}_{3} \mathrm{~N}$ and $\mathrm{C}_{2} \mathrm{H}_{3} \mathrm{CN}$, or the direct grain-surface addition of pre-formed large hydrocarbon radicals like $\mathrm{C}_{2} \mathrm{H}_{5}$ or $\mathrm{C}_{3} \mathrm{H}_{7}$ to a $\mathrm{CN}$ radical - appear to be very much too fast, resulting in excessive quantities of the two largest alkyl cyanides.

To achieve the appropriate ratios, those two formation routes must be artificially disabled within the model. Why should these mechanisms be less efficient in reality than they would appear from the model? Firstly, gas-phase $\mathrm{HC}_{3} \mathrm{~N}$ and $\mathrm{C}_{2} \mathrm{H}_{3} \mathrm{CN}$ may be less abundant than the model suggests. The evaporation, and subsequent reaction, of $\mathrm{HCN}$ from the grains is a primary cause of gas-phase formation for each of these molecules. Variation in the evaporation characteristics or the composition/structure of the ices may weaken such mechanisms. However, the agreement between observed and modeled abundances of vinyl cyanide is very good. Indeed, the Select model shows excellent agreement, providing further justification for the omission of its hydrogenation reactions.

Alternatively, surface hydrogenation of $\mathrm{HC}_{3} \mathrm{~N}$ and $\mathrm{C}_{2} \mathrm{H}_{3} \mathrm{CN}$, once they have accreted onto the grains, may be less efficient than has been assumed here. Importantly, activation energies are required for hydrogenation of both these species, whose values are poorly constrained. The fact that it is these very reactions that must be disabled suggests strongly that their activation energies should be significantly higher than has been assumed here. Additionally, our use of a "deterministic" gasgrain model may also produce somewhat more efficient hydrogenation than is really the case (although a test-run using the rate-modification method of Garrod 2008 shows no great difference in this respect).

In the case of the addition of large hydrocarbon radicals to $\mathrm{CN}$, the over-dominance of these channels is probably due to the incompleteness of the hydrocarbon chemistry as a whole, particularly on the grains. Whilst up to 10 carbon atoms in a chain are considered in this model, the hydrogenation states of the larger chains are typically limited to 4 hydrogen atoms. Crucially, hydrogenation is the only type of reaction included in the network for most hydrocarbons, aside from the newly-added $\mathrm{CN}$ addition reactions. The hydrocarbon reaction set was largely devised with cold dark clouds in mind, where hydrogenation dominates. By including only a single new reaction (addition to $\mathrm{CN}$ ) for any particular hydrocarbon, that reaction can easily become the dominant channel. The completion of the hydrocarbon network to include reactions with all major reactants would be beneficial, although this is not a trivial task.

The small hydrocarbons $\mathrm{CH}_{2}$ and $\mathrm{CH}_{3}$, on the other hand, as well as $\mathrm{CN}$ itself, have a much more comprehensive reaction network, making sequential addition and its apparent degree of efficiency more credible.

Ethyl formate and aminoacetonitrile also seem to be well reproduced with a similar addition scheme to that of the alkyl cyanides. Ethyl formate abundance may be dependent on ethanol as well as methyl formate, depending on the specific conditions.

The Select model reproduces well the abundance ratios for alkyl cyanides, but their absolute abundances are an order of magnitude lower than observational values. This also results in a poor match to abundance ratios relative to methyl formate and other methanol-related species. In fact, the chemistries of the cyanides and the methanol-related species do not strongly influence one another in the model. The overall abundances of each category of molecule are mainly influenced by different, independent parameters: the formation rate of the products of methanol photodissociation (i.e. the product of the photodissociation rate and absolute grain-surface abundance of methanol), and the quantity of HCN or related nitrile-group species in the ice mantles, respectively. Similarly, the modeled abundance of aminoacetonitrile relative to the alkyl cyanides is very high. The 
formation rate of this molecule is strongly dependent on the product of the abundance of $\mathrm{NH}_{3}$ in the ices, and its rate of photodissociation. This indicates that one or both of these values may be too large, by at least an order of magnitude. A parameter search should yield the optimal values for all such quantities, but such is not the focus of this paper.

The augmentation of methyl formate binding energy allows its abundance to remain high at temperatures appropriate to the densest parts of the hot core. However, the low observed rotational temperatures suggest that methyl formate should still have a binding energy less than that of $\mathrm{H}_{2} \mathrm{O}$, which is indeed the case here, even with the highest value we use. A value somewhat lower than our maximum would also achieve quite acceptable results. Clearly, an experimental value for binding to astrophysically appropriate surfaces would be highly valuable for the chemical modeling of hot cores.

While certain crucial steps in the formation of these complex molecules occur only in the gas-phase or on the grain surfaces, processes in each phase are inter-dependent and cannot be understood in isolation.

\section{Conclusions}

We used the complete $3 \mathrm{~mm}$ and partial 2 and $1.3 \mathrm{~mm}$ line surveys obtained with the IRAM $30 \mathrm{~m}$ telescope toward the hot cores Sgr B2(N) and (M) to search for emission from the organic molecules ethyl formate and $n$-propyl cyanide. We report the detection of both molecules toward the hot core Sgr B2(N), which are the first detections of these molecules in the interstellar medium. Our main results and conclusions are the following:

1. New entries for the CDMS catalog have been created for $n$-propyl cyanide and ethyl formate.

2. 46 of the 711 significant transitions of the anti-conformer of ethyl formate covered by our $30 \mathrm{~m}$ line survey are relatively free of contamination from other molecules and are detected in the form of 24 observed features toward Sgr B2(N). The emission of the gauche-conformer is too weak to be clearly detected in our survey.

3. 50 of the 636 significant transitions of the anti-conformer of $n$-propyl cyanide covered by our $30 \mathrm{~m}$ line survey are relatively free of contamination from other molecules and are detected in the form of 12 observed features toward Sgr B2(N) with two velocity components. The emission of the gaucheconformer is too weak to be clearly detected in our survey.

4. With a source size of $3^{\prime \prime}$, we derive an ethyl formate column density of $5.4 \times 10^{16} \mathrm{~cm}^{-2}$ for a temperature of $100 \mathrm{~K}$ and a linewidth of $7 \mathrm{~km} \mathrm{~s}^{-1}$ in the LTE approximation. The abundance of ethyl formate relative to $\mathrm{H}_{2}$ is estimated to be $3.6 \times 10^{-9}$

5. The two velocity components detected in $n$-propyl cyanide have LTE column densities of $1.5 \times 10^{16}$ and $6.6 \times$ $10^{15} \mathrm{~cm}^{-2}$, respectively, with a temperature of $150 \mathrm{~K}$, a linewidth of $7 \mathrm{~km} \mathrm{~s}^{-1}$, and a source size of $3^{\prime \prime}$. The fractional abundance of $n$-propyl cyanide in the main source is estimated to be $1.0 \times 10^{-9}$.

6. We detected neither ethyl formate nor $n$-propyl cyanide toward the more evolved source Sgr B2(M) and derived column density upper limits of $2 \times 10^{16}$ and $6 \times 10^{15} \mathrm{~cm}^{-2}$, respectively, for a source size of $3^{\prime \prime}$.

7. We modeled the emission of chemically related species also detected in our survey of Sgr B2(N) and derived column density ratios of $0.8 / 15 / 1$ for $t-\mathrm{HCOOH} / \mathrm{CH}_{3} \mathrm{OCHO} /$
$\mathrm{C}_{2} \mathrm{H}_{5} \mathrm{OCHO}$ and $108 / 80 / 1$ for $\mathrm{CH}_{3} \mathrm{CN} / \mathrm{C}_{2} \mathrm{H}_{5} \mathrm{CN} / \mathrm{C}_{3} \mathrm{H}_{7} \mathrm{CN}$ in the main hot core of Sgr B2(N).

8. The chemical models suggest that the sequential, piecewise construction of ethyl and $n$-propyl cyanide from their constituent functional groups on the grain surfaces is their most likely formation route. Aminoacetonitrile formation proceeds similarly, suggesting a possible correlation with ethyl cyanide abundance. Vinyl cyanide is formed predominantly in the gas-phase.

9. Comparison of the observational and model results suggests that the production of alkyl cyanides by the hydrogenation of less saturated species is much less efficient than functionalgroup addition.

10. Ethyl formate can be formed on the grains by addition of $\mathrm{HCO}$ or $\mathrm{CH}_{3}$ to functional-group radicals derived from methyl formate and ethanol; however, methyl formate appears to be the dominant precursor.

11. Understanding of the complex interactions between gasphase and grain-surface processes may be necessary to fully explain the observational features displayed by many complex molecules, including formic acid and methyl formate.

12. The detection in Sgr B2(N) of the next stage of complexity in two classes of complex molecule, esters and alkyl cyanides, suggests that greater complexity also may be present in other classes of molecule in the interstellar medium.

Our results have demonstrated the power of the "complete spectrum fitting" approach used by us as a technique that is mandatory today for the identification of new complex molecules by their generally weak signals. Ideally, one would want to verify identifications with interferometric observations as done for the case of aminoacetonitrile (Belloche et al. 2008a,b). However, given the limited collecting area, bandwidth and spatial resolution of today's interferometer arrays, this would be very time consuming or even prohibitive. It will, however, be a trivial exercise for the Atacama Large Millimeter Array (ALMA) once it is fully operational.

Acknowledgements. We thank the anonymous referee and the editor for their careful reading of the manuscript and for their suggestions that helped improve the clarity of this article. H.S.P.M. thanks Dr. Jürgen Aschenbach from the library of the University of Kiel for providing the supplementary material to Vormann \& Dreizler (1988). We are grateful to Eric Herbst for providing the ethyl formate spectroscopic line list as well as a preprint of the manuscript prior to publication. H.S.P.M. thanks the Deutsche Forschungsgemeinschaft (DFG) for initial support through the collaborative research grant SFB 494. He is grateful to the Bundesministerium für Bildung und Forschung (BMBF) for recent support which was administered through Deutsches Zentrum für Luft- und Raumfahrt (DLR). R.T.G thanks the Alexander von Humboldt Foundation for a Research Fellowship.

\section{References}

Belloche, A., Menten, K. M., Comito, C., et al. 2008a, A\&A, 482, 179 Belloche, A., Menten, K. M., Comito, C., et al. 2008b, A\&A, 492, 769 Bernstein, M. P., Dworkin, J. P., Sandford, S. A., Cooper, G. W., \& Allamandola, L. J. 2002, Nature, 416, 401

Carty, D., Le Page, V., Sims, I. R., \& Sims, I. W. M. 2001, Chem. Phys. Lett., 344,310

Comito, C., Schilke, P., Phillips, T. G., et al. 2005, ApJS, 156, 127

Demaison, J., \& Dreizler, H. 1982, Z. Naturforsch. A, 37, 199

Demaison, J., Boucher, D., Burie, J., \& Dubrulle, A. 1984, Z. Naturforsch. A, 39,560

de Vicente, P., Martin-Pintado, J., \& Wilson, T. L. 1997, A\&A, 320, 957

Ehrenfreund, P., Glavin, D. P., Botta, O., Cooper, G., \& Bada, J. L. 2001, Proceedings of the National Academy of Science, 98, 2138

Elsila, J. E., Dworkin, J. P., Bernstein, M. P., Martin, M. P., \& Sandford, S. A. 2007, ApJ, 660, 911

Friedel, D. N., Snyder, L. E., Turner, B. E., \& Remijan, A. 2004, ApJ, 600, 234 
Garrod, R. T. 2008, A\&A, 491, 239

Garrod, R. T., \& Herbst, E. 2006, A\&A, 457, 927

Garrod, R. T., Widicus Weaver, S. L., \& Herbst, E. 2008, ApJ, 682, 283

Geppert, W. D., Hamberg, M., Thomas, R. D., et al. 2006, Faraday Discuss., 133, 177

Goldsmith, P. F., \& Langer, W. D. 1999, ApJ, 517, 209

Gordon, M. A., Berkermann, U., Mezger, P. G., et al. 1993, A\&A, 280, 208

Hirota, E. 1962, J. Chem. Phys., 37, 2918

Hollis, J. M., Lovas, F. J., \& Jewell, P. R. 2000, ApJ, 540, L107

Hollis, J. M., Pedelty, J. A., Boboltz, D. A., et al. 2003, ApJ, 596, L235

Hollis, J. M., Lovas, F. J., Remijan, A. J., et al. 2006, ApJ, 643, L25

Horn, A., Møllendal, H., Sekiguchi, O., et al. 2004, ApJ, 611, 605

Jones, P. A., Cunningham, M. R., Godfrey, P. D., \& Cragg, D. M. 2007, MNRAS, 374,579

Jones, P. A., Burton, M. G., Cunningham, M. R., et al. 2008, MNRAS, 386, 117 Lis, D. C., \& Goldsmith, P. F. 1989, ApJ, 337, 704

Lis, D. C., Goldsmith, P. F., Carlstrom, J. E., \& Scoville, N. Z. 1993, ApJ, 402, 238

Liu, S.-Y., \& Snyder, L. E. 1999, ApJ, 523, 683

Liu, S.-Y., Mehringer, D. M., \& Snyder, L. E. 2001, ApJ, 552, 654

Medvedev, I. R., De Lucia, F. C., \& Herbst, E. 2009, ApJS, 181, 433

Mehringer, D. M., Snyder, L. E., Miao, Y., \& Lovas, F. J. 1997, ApJ, 480, L71

Miao, Y., Mehringer, D. M., Kuan, Y.-J., \& Snyder, L. E. 1995, ApJ, 445, L59
Millar, T. J., Herbst, E., \& Charnley, S. B. 1991, ApJ, 369, 147

Müller, H. S. P., Thorwirth, S., Roth, D. A., \& Winnewisser, G. 2001, A\&A, 370, L49

Müller, H. S. P., Schlöder, F., Stutzki, J., \& Winnewisser, G. 2005, J. Mol. Struct, 742,215

Müller, H. S. P., Belloche, A., Menten, K. M., Comito, C., \& Schilke, P. 2008, J. Mol. Spectrosc., 251, 319

Nummelin, A., Bergman, P., Hjalmarson, Å., et al. 2000, ApJS, 128, 213

Osorio, M., Lizano, S., \& D’Alessio, P. 1999, ApJ, 525, 808

Pearson, J. C., Brauer, C. S., \& Drouin, B. J. 2008, J. Mol. Spectrosc., 251, 394 Pickett, H. M., Poynter, I. R. L., Cohen, E. A., et al. 1998, J. Quant. Spectrosc. Radiat. Transfer, 60, 883

Reid, M. J. 1993, ARA\&A, 31, 345

Riveros, J. M., \& Bright Wilson, E., Jr. 1967, J. Chem. Phys., 46, 4605

Snyder, L. E., Kuan, Y.-J., \& Miao, Y. 1994, The Structure and Content of Molecular Clouds, ed. T. L. Wilson, \& K. J. Johnston (Springer-Verlag), Lecture Notes in Physics, 439, 187

Snyder, L. E., Lovas, F. J., Hollis, J. M., et al. 2005, ApJ, 619, 914

Vormann, K., \& Dreizler, H. 1988, Z. Naturforsch. A, 43, 338

Włodarczak, G., Martinache, L., Demaison, J., Marstokk, K.-M., \& Møllendal, H. 1988, J. Mol. Spectrosc., 127, 178

Włodarczak, G., Martinache, L., Demaison, J., Marstokk, K.-M., \& Møllendal, H. 1991, J. Mol. Spectrosc., 146, 224 
A. Belloche et al.: Detection and chemical modeling of ethyl formate and $n$-propyl cyanide in Sgr B2(N), Online Material $p 1$

Table 1. Transitions of the anti-conformer of ethyl formate observed with the IRAM $30 \mathrm{~m}$ telescope toward Sgr B2(N). The horizontal lines mark discontinuities in the observed frequency coverage. Only the transitions associated with a modeled line stronger than $20 \mathrm{mK}$ are listed.

\begin{tabular}{|c|c|c|c|c|c|c|c|}
\hline$N^{a}$ & Transition $^{b}$ & $\begin{array}{c}\text { Frequency } \\
(\mathrm{MHz}) \\
(3) \\
\end{array}$ & $\begin{array}{c}\text { Unc. }^{c} \\
(\mathrm{kHz}) \\
(4) \\
\end{array}$ & $\begin{array}{l}E_{1}{ }^{d} \\
(\mathrm{~K}) \\
(5) \\
\end{array}$ & $\begin{array}{c}S \mu^{2} \\
\left(\mathrm{D}^{2}\right) \\
(6) \\
\end{array}$ & $\begin{array}{c}\sigma^{e} \\
(\mathrm{mK}) \\
(7) \\
\end{array}$ & $\begin{array}{c}\text { Comments } \\
(8) \\
\end{array}$ \\
\hline 1 & $15_{2,14}-14_{2,13}$ & 81779.567 & 4 & 30 & 50.5 & 13 & Detected, blend with $U$-line \\
\hline 2 & 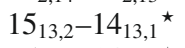 & 82297.815 & 4 & 149 & 12.8 & 19 & Blend with $\mathrm{C}_{2} \mathrm{H}_{5} \mathrm{CN}, v_{13}=1 / v_{21}=1$ and absorption line of $\mathrm{c}-\mathrm{HC}^{13} \mathrm{CCH}$ \\
\hline 4 & 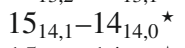 & 82297.944 & 4 & 169 & 6.6 & 19 & Blend with $\mathrm{C}_{2} \mathrm{H}_{5} \mathrm{CN}, v_{13}=1 / v_{21}=1$ and absorption line of $\mathrm{c}-\mathrm{HC}{ }^{13} \mathrm{CCH}$ \\
\hline 6 & $15_{12,3}-14_{12,2^{\star}}$ & 82298.480 & 4 & 131 & 18.5 & 19 & Blend with $\mathrm{C}_{2} \mathrm{H}_{5} \mathrm{CN}, v_{13}=1 / v_{21}=1$ and absorption line of $\mathrm{c}-\mathrm{HC}{ }^{13} \mathrm{CCH}$ \\
\hline 8 & $15_{10,5}-14_{10,4}{ }^{\star}$ & 82303.360 & 4 & 100 & 28.6 & 19 & Blend with $\mathrm{HC}_{3} \mathrm{~N}, v_{6}=v_{7}=1$ and absorption line of $\mathrm{c}-\mathrm{HC}^{13} \mathrm{CCH}$ \\
\hline 10 & $15_{9,6}-14_{9,5}^{\star}$ & 82308.580 & 4 & 86 & 32.9 & 19 & Strong $\mathrm{HC}_{3} \mathrm{~N}, v_{6}=v_{7}=1$ \\
\hline 12 & $15_{8,7}-14_{8,6}{ }^{\star}$ & 82316.890 & 4 & 74 & 36.8 & 19 & Strong $\mathrm{HC}_{3} \mathrm{~N}, v_{6}=v_{7}=1$ \\
\hline 14 & $15_{7,8}-14_{7,7}^{\star}$ & 82330.125 & 4 & 63 & 40.2 & 19 & Blend with $\mathrm{CH}_{3} \mathrm{CHO}$ and $\mathrm{CH}_{3}{ }^{18} \mathrm{OH}$ \\
\hline 16 & $15_{6,10}-14_{6,9}{ }^{\star}$ & 82351.854 & 4 & 54 & 43.2 & 19 & Detected, partial blend with $\mathrm{C}_{2} \mathrm{H}_{5} \mathrm{CN}, v_{13}=1 / v_{21}=1$ and $U$-line \\
\hline 18 & $19_{3,17}-19_{2,18}$ & 82389.204 & 9 & 53 & 4.8 & 18 & Blend with ${ }^{13} \mathrm{CH}_{3} \mathrm{OH}$ \\
\hline 19 & $15_{5,11}-14_{5,10}$ & 82389.698 & 4 & 46 & 45.7 & 18 & Blend with ${ }^{13} \mathrm{CH}_{3} \mathrm{OH}$ \\
\hline 20 & $15_{5,10}-14_{5,9}$ & 82390.016 & 4 & 46 & 45.7 & 18 & Blend with ${ }^{13} \mathrm{CH}_{3} \mathrm{OH}$ \\
\hline 21 & $15_{4,12}-14_{4,11}$ & 82457.126 & 4 & 39 & 47.8 & 18 & Strong $\mathrm{CH}_{3} \mathrm{OCH}_{3}$ \\
\hline 22 & $15_{4,11}-14_{4,10}$ & 82471.367 & 4 & 39 & 47.8 & 18 & Blend with $\mathrm{CH}_{2}(\mathrm{OH}) \mathrm{CHO}$ and $U$-line \\
\hline 23 & $15_{3,13}-14_{3,12}$ & 82485.618 & 4 & 34 & 49.4 & 18 & Blend with $U$-lines \\
\hline 24 & $15_{3,12}-14_{3,11}$ & 82802.132 & 4 & 34 & 49.4 & 17 & Blend with $\mathrm{C}_{2} \mathrm{H}_{5} \mathrm{CN}$ \\
\hline 25 & $15_{1,14}-14_{1,13}$ & 83792.699 & 4 & 29 & 51.1 & 16 & Blend with $\mathrm{CH}_{3} \mathrm{OH}$ and $U$-lines \\
\hline 26 & $15_{2,13}-14_{2,12}$ & 84081.357 & 4 & 31 & 50.5 & 19 & Detected, partial blend with $\mathrm{CH}_{3} \mathrm{CH}_{3} \mathrm{CO}, v_{t}=1$ \\
\hline 27 & $16_{1,16}-15_{1,15}$ & 84444.095 & 4 & 31 & 54.6 & 19 & Blend with ${ }^{13} \mathrm{CH}_{3} \mathrm{OH}$ and $\mathrm{C}_{2} \mathrm{H}_{5} \mathrm{CN}, v_{13}=1 / v_{21}=1$ \\
\hline 28 & $16_{0,16}-15_{0,15}$ & 85065.106 & 4 & 31 & 54.7 & 22 & Detected, partial blend with $\mathrm{c}-\mathrm{C}_{2} \mathrm{H}_{4} \mathrm{O}$ \\
\hline 29 & $16_{2,15}-15_{2,14}$ & 87160.417 & 4 & 34 & 54.0 & 17 & Strong $\mathrm{CH}_{3} \mathrm{OCHO}$ \\
\hline 30 & $16_{14,2}-15_{14,1} \star$ & 87785.462 & 4 & 173 & 12.9 & 17 & Blend with $\mathrm{C}_{2} \mathrm{H}_{5} \mathrm{CN}, v_{13}=1 / v_{21}=1$, problem with baseline? \\
\hline 32 & $16_{13,3}-15_{13,2}{ }^{\star}$ & 87785.683 & 4 & 153 & 18.7 & 17 & Blend with $\mathrm{C}_{2} \mathrm{H}_{5} \mathrm{CN}, v_{13}=1 / v_{21}=1$, problem with baseline? \\
\hline 34 & $16_{15,1}-15_{15,0} \star$ & 87785.966 & 4 & 193 & 6.6 & 17 & Blend with $\mathrm{C}_{2} \mathrm{H}_{5} \mathrm{CN}, v_{13}=1 / v_{21}=1$, problem with baseline? \\
\hline 36 & $16_{12,4}-15_{12,3}{ }^{\star}$ & 87786.848 & 4 & 135 & 24.0 & 17 & Blend with $\mathrm{C}_{2} \mathrm{H}_{5} \mathrm{CN}, v_{13}=1 / v_{21}=1$, problem with baseline? \\
\hline 38 & $16_{11,5}-15_{11,4}^{\star}$ & 87789.267 & 4 & 119 & 28.9 & 17 & Blend with $U$-line, uncertain baseline \\
\hline 40 & $16_{10,6}-15_{10,5}{ }^{\star}$ & 87793.413 & 4 & 104 & 33.4 & 17 & Blend with $\mathrm{CH}_{3}{ }^{13} \mathrm{CH}_{2} \mathrm{CN}$ and $U$-line \\
\hline 42 & $16_{9,7}-15_{9,6^{\star}}$ & 87800.031 & 4 & 90 & 37.5 & 17 & Blend with $\mathrm{CH}_{3}{ }^{13} \mathrm{CH}_{2} \mathrm{CN}$ and $\mathrm{HNCO}, v_{4}=1$ \\
\hline 44 & $16_{8,8}-15_{8,7}^{\star}$ & 87810.372 & 4 & 78 & 41.2 & 17 & Group detected, partial blend with $\mathrm{CH}_{2}(\mathrm{OH}) \mathrm{CHO}$ and $\mathrm{C}_{2} \mathrm{H}_{5} \mathrm{CN}$ \\
\hline 46 & $16_{7,9}-15_{7,8}^{\star}$ & 87826.665 & 4 & 67 & 44.4 & 17 & Group detected, partial blend with $\mathrm{HNCO}, v_{5}=1$ \\
\hline 48 & $16_{6,11}-15_{6,10}{ }^{\star}$ & 87853.244 & 4 & 58 & 47.2 & 17 & Blend with $\mathrm{NH}_{2} \mathrm{CHO}$, problem with baseline? \\
\hline 50 & $16_{5,12}-15_{5,11}$ & 87899.302 & 4 & 50 & 49.5 & 17 & Strong $\mathrm{C}_{2} \mathrm{H}_{5}{ }^{13} \mathrm{CN}$, $\mathrm{HNCO}$, and $\mathrm{HN}^{13} \mathrm{CO}$ \\
\hline 51 & $16_{5,11}-15_{5,10}$ & 87899.879 & 4 & 50 & 49.5 & 17 & Strong $\mathrm{C}_{2} \mathrm{H}_{5}{ }^{13} \mathrm{CN}$, $\mathrm{HNCO}$, and $\mathrm{HN}^{13} \mathrm{CO}$ \\
\hline 52 & $16_{4,13}-15_{4,12}$ & 87979.119 & 4 & 43 & 51.4 & 19 & Blend with $U$-lines \\
\hline 53 & $16_{3,14}-15_{3,13}$ & 87993.944 & 4 & 38 & 52.9 & 19 & Detected, partial blend with $\mathrm{CH}_{3} \mathrm{CH}_{3} \mathrm{CO}$ and $U$-line \\
\hline 54 & $16_{4,12}-15_{4,11}$ & 88001.562 & 4 & 43 & 51.4 & 19 & Detected, blend with $\mathrm{C}_{2} \mathrm{H}_{5} \mathrm{CN}, v_{13}=1 / v_{21}=1$ \\
\hline 55 & $16_{3,13}-15_{3,12}$ & 88425.192 & 4 & 38 & 52.9 & 17 & Blend with $\mathrm{CH}_{2}(\mathrm{OH}) \mathrm{CHO}$ \\
\hline 56 & $16_{1,15}-15_{1,14}$ & 89218.355 & 4 & 33 & 54.6 & 16 & Strong $\mathrm{HCO}^{+}$in absorption \\
\hline 57 & $17_{1,17}-16_{1,16}$ & 89651.925 & 4 & 35 & 58.0 & 18 & Blend with $\mathrm{C}_{2} \mathrm{H}_{5} \mathrm{CN}, v_{13}=1 / v_{21}=1, \mathrm{CH}_{3} \mathrm{OH}, v_{t}=2$, and $\mathrm{C}_{2} \mathrm{H}_{5} \mathrm{CN}, v_{20}=1$ \\
\hline 58 & $16_{2,14}-15_{2,13}$ & 89804.728 & 4 & 35 & 54.0 & 18 & Blend with $\mathrm{CH}_{3} \mathrm{CH}_{3} \mathrm{CO}$ \\
\hline 59 & $17_{0,17}-16_{0,16}$ & 90190.497 & 5 & 35 & 58.1 & 17 & Blend with $\mathrm{CH}_{2}(\mathrm{OH}) \mathrm{CHO}, \mathrm{C}_{2} \mathrm{H}_{5} \mathrm{CN}, v_{13}=1 / v_{21}=1$, and $U$-line \\
\hline 60 & $17_{2,16}-16_{2,15}$ & 92528.452 & 4 & 39 & 57.4 & 22 & Blend with $\mathrm{CH}_{3} \mathrm{CN}, v_{8}=2$ \\
\hline 61 & $17_{14,3}-16_{14,2}{ }^{\star}$ & 93273.169 & 4 & 177 & 18.8 & 22 & Blend with $U$-lines \\
\hline 63 & $17_{15,2}-16_{15,1^{\star}}$ & 93273.377 & 5 & 198 & 12.9 & 22 & Blend with $U$-lines \\
\hline 65 & $17_{13,4}-16_{13,3}{ }^{\star}$ & 93273.813 & 4 & 157 & 24.2 & 22 & Blend with $U$-lines \\
\hline 67 & $17_{16,1}-16_{16,0}{ }^{\star}$ & 93274.251 & 5 & 220 & 6.7 & 22 & Blend with $U$-lines \\
\hline 69 & $17_{12,5}-16_{12,4}$ * & 93275.564 & 4 & 139 & 29.3 & 22 & Blend with $U$-lines \\
\hline 71 & $17_{11,6}-16_{11,5}{ }^{\star}$ & 93278.797 & 4 & 123 & 33.9 & 22 & Blend with $U$-lines \\
\hline 73 & $17_{10,7}-16_{10,6}{ }^{\star}$ & 93284.077 & 4 & 108 & 38.1 & 22 & Group detected, uncertain baseline \\
\hline 75 & $17_{9,8}-16_{9,7^{\star}}$ & 93292.297 & 4 & 94 & 42.0 & 22 & Group detected, uncertain baseline \\
\hline 77 & $17_{8,9}-16_{8,8}{ }^{\star}$ & 93304.955 & 4 & 82 & 45.4 & 22 & Group detected, partial blend with $U$-line \\
\hline 79 & $17_{7,11}-16_{7,10}^{\star}$ & 93324.728 & 4 & 71 & 48.4 & 22 & Group detected, partial blend with $U$-lines \\
\hline 81 & $17_{6,12}-16_{6,11}^{\star}$ & 93356.821 & 4 & 62 & 51.0 & 22 & Group detected, blend with $\mathrm{CH}_{3} \mathrm{CH}_{3} \mathrm{CO}$ and $U$-line? \\
\hline 83 & $17_{5,13}-16_{5,12}$ & 93412.160 & 4 & 54 & 53.3 & 22 & Group detected, partial blend with $U$-line? \\
\hline 84 & $17_{5,12}-16_{5,11}$ & 93413.168 & 4 & 54 & 53.3 & 22 & Group detected, partial blend with $U$-line? \\
\hline 85 & $17_{3,15}-16_{3,14}$ & 93499.096 & 4 & 42 & 56.5 & 24 & Blend with $\mathrm{C}_{2} \mathrm{H}_{5} \mathrm{OH}$ and $U$-line \\
\hline 86 & $17_{4,14}-16_{4,13}$ & 93504.972 & 4 & 47 & 55.1 & 24 & Detected, blend with $\mathrm{CH}_{3}{ }^{18} \mathrm{OH}$ \\
\hline 87 & $174,13-16_{4,12}$ & 93539.303 & 4 & 47 & 55.1 & 24 & Detected \\
\hline 88 & $17_{3,14}-16_{3,13}$ & 94072.891 & 4 & 42 & 56.5 & 31 & Blend with $\mathrm{CH}_{3} \mathrm{CH}_{3} \mathrm{CO}, v_{t}=1$ and ${ }^{13} \mathrm{CH}_{3} \mathrm{OH}, v_{t}=1$ \\
\hline 89 & $17_{1,16}-16_{1,15}$ & 94608.131 & 4 & 37 & 58.0 & 26 & Blend with ${ }^{13} \mathrm{CH}_{3} \mathrm{CH}_{2} \mathrm{CN}$ and $U$-line \\
\hline 90 & $18_{1,18}-17_{1,17}$ & 94852.542 & 5 & 40 & 61.5 & 28 & Weak \\
\hline 91 & $18_{0,18}-17_{0,17}$ & 95313.428 & 5 & 39 & 61.5 & 28 & Weak, partial blend with $\mathrm{C}_{2} \mathrm{H}_{3} \mathrm{CN}, v_{11}=1 / v_{15}=1$, uncertain baseline \\
\hline 92 & $17_{2,15}-16_{2,14}$ & 95517.316 & 4 & 39 & 57.5 & 23 & Blend with $\mathrm{C}_{2} \mathrm{H}_{3} \mathrm{CN}, v_{11}=2$ \\
\hline
\end{tabular}


Table 1. continued.

\begin{tabular}{|c|c|c|c|c|c|c|c|}
\hline$N^{a}$ & Transition $^{b}$ & $\begin{array}{l}\text { Frequency } \\
\text { (MHz) } \\
\text { (3) }\end{array}$ & $\begin{array}{c}\text { Unc. }^{c} \\
(\mathrm{kHz}) \\
(4)\end{array}$ & $\begin{array}{l}E_{1}^{d} \\
(\mathrm{~K}) \\
(5)\end{array}$ & $\begin{array}{c}S \mu^{2} \\
\left(\mathrm{D}^{2}\right) \\
(6)\end{array}$ & $\begin{array}{c}\sigma^{e} \\
(\mathrm{mK}) \\
(7)\end{array}$ & Comments \\
\hline 93 & $18_{2,17}-17_{2,16}$ & 97883.281 & 4 & 43 & 60.9 & 20 & Blend with $\mathrm{CH}_{3} \mathrm{OCHO}, v_{\mathrm{t}}=1$ and $\mathrm{NH}_{2} \mathrm{CHO}$ \\
\hline 94 & $18_{15,3}-17_{15,2^{\star}}$ & 98760.931 & 5 & 202 & 18.9 & 18 & Group detected, blend with $U$-line \\
\hline 96 & $18_{14,4}-17_{14,3}^{\star}$ & 98761.079 & 5 & 181 & 24.4 & 18 & Group detected, blend with $U$-line \\
\hline 98 & $18_{16,2}-17_{16,1}^{\star}$ & 98761.555 & 5 & 224 & 13.0 & 18 & Group detected, blend with $U$-line \\
\hline 100 & $18_{13,5}-17_{13,4}^{\star}$ & 98762.218 & 5 & 162 & 29.5 & 18 & Group detected, blend with $U$-line \\
\hline 102 & $18_{17,1}-17_{17,0}^{\star}$ & 98762.790 & 5 & 248 & 6.7 & 18 & Group detected, blend with $U$-line \\
\hline 104 & $18_{12,6}-17_{12,5}^{\star}$ & 98764.650 & 5 & 144 & 34.3 & 18 & Group detected \\
\hline 106 & $18_{11,7}-17_{11,6}^{\star}$ & 98768.818 & 5 & 127 & 38.7 & 18 & Blend with $U$-line \\
\hline 108 & $18_{10,8}-17_{10,7^{\star}}$ & 98775.390 & 5 & 112 & 42.7 & 18 & Blend with $\mathrm{C}_{2} \mathrm{H}_{5} \mathrm{CN}, v_{20}=1$ \\
\hline 110 & $18_{9,9}-17_{9,8^{\star}}$ & 98785.427 & 5 & 99 & 46.3 & 18 & Blend with $U$-line, $\mathrm{CH}_{3} \mathrm{OCHO}$, and $\mathrm{C}_{2} \mathrm{H}_{5} \mathrm{CN}, v_{13}=1 / v_{21}=1$ \\
\hline 112 & $18_{8,10}-17_{8,9}{ }^{\star}$ & 98800.707 & 5 & 86 & 49.6 & 18 & Blend with $\mathrm{CH}_{3} \mathrm{CH}_{3} \mathrm{CO}$ \\
\hline 114 & $18_{7,12}-17_{7,11}^{\star}$ & 98824.411 & 4 & 76 & 52.4 & 18 & Blend with $\mathrm{C}_{2} \mathrm{H}_{5} \mathrm{OH}$ \\
\hline 116 & $18_{6,13}-17_{6,12}{ }^{\star}$ & 98862.724 & 4 & 66 & 54.9 & 18 & Blend with strong $U$-line \\
\hline 118 & $18_{5,14}-17_{5,13}$ & 98928.453 & 4 & 58 & 57.0 & 18 & Group detected, blend with $\mathrm{C}_{2} \mathrm{H}_{5} \mathrm{CN}, v_{13}=1 / v_{21}=1$ \\
\hline 119 & $18_{5,13}-17_{5,12}$ & 98930.153 & 4 & 58 & 57.0 & 18 & Group detected, blend with $\mathrm{C}_{2} \mathrm{H}_{5} \mathrm{CN}, v_{13}=1 / v_{21}=1$ \\
\hline 120 & $18_{3,16}-17_{3,15}$ & 98999.806 & 4 & 47 & 60.0 & 18 & Blend with $U$-lines \\
\hline 121 & $18_{4,15}-17_{4,14}$ & 99034.515 & 4 & 52 & 58.7 & 19 & Blend with ${ }^{13} \mathrm{CH}_{3} \mathrm{CH}_{2} \mathrm{CN}, \mathrm{CH}_{3} \mathrm{CH}_{3} \mathrm{CO}, v_{t}=1$, and $U$-lines \\
\hline 122 & $18_{4,14}-17_{4,13}$ & 99085.662 & 4 & 52 & 58.7 & 19 & Blend with $\mathrm{a}\left(\mathrm{CH}_{2} \mathrm{OH}\right)_{2}$ and $\mathrm{CH}_{3} \mathrm{OCHO}, v_{\mathrm{t}}=1$ \\
\hline 123 & $18_{3,15}-17_{3,14}$ & 99746.684 & 5 & 47 & 60.0 & 14 & Blend with $\mathrm{NH}_{2} \mathrm{CN}$ and $\mathrm{C}_{2} \mathrm{H}_{5} \mathrm{CN}, v_{20}=1$ \\
\hline 124 & $18_{1,17}-17_{1,16}$ & 99959.814 & 4 & 42 & 61.4 & 14 & Blend with $\mathrm{NH}_{2} \mathrm{CN}$ and $\mathrm{CH}_{3} \mathrm{CH}_{3} \mathrm{CO}$ \\
\hline 125 & $19_{1,19}-18_{1,18}$ & 100046.587 & 5 & 44 & 64.9 & 14 & Blend with $\mathrm{HCC}^{13} \mathrm{CN}, v_{7}=1$ and $U$-line \\
\hline 126 & $19_{0,19}-18_{0,18}$ & 100436.418 & 5 & 44 & 64.9 & 24 & Blend with $\mathrm{CH}_{3} \mathrm{OCH}_{3}$ and $\mathrm{C}_{2} \mathrm{H}_{5} \mathrm{OH}$ \\
\hline 127 & $18_{2,16}-17_{2,15}$ & 101213.969 & 5 & 44 & 61.0 & 21 & Blend with $U$-line \\
\hline 128 & $19_{2,18}-18_{2,17}$ & 103224.624 & 5 & 48 & 64.4 & 25 & Blend with $\mathrm{c}-\mathrm{C}_{2} \mathrm{H}_{4} \mathrm{O}, \mathrm{CH}_{3} \mathrm{OCHO}$, and $U$-line \\
\hline 129 & $19_{15,4}-18_{15,3^{\star}}$ & 104248.635 & 5 & 207 & 24.6 & 48 & Blend with $\mathrm{SO}_{2}$ and $U$-line? \\
\hline 131 & $19_{16,3}-18_{16,2}{ }^{\star}$ & 104248.958 & 5 & 229 & 19.0 & 48 & Blend with $\mathrm{SO}_{2}$ and $U$-line? \\
\hline 133 & $19_{14,5}-18_{14,4}^{\star}$ & 104249.203 & 5 & 186 & 29.8 & 48 & Blend with $\mathrm{SO}_{2}$ and $U$-line? \\
\hline 135 & $19_{17,2}-18_{17,1^{\star}}^{\star}$ & 104249.983 & 5 & 253 & 13.0 & 48 & Blend with $\mathrm{SO}_{2}$ and $U$-line? \\
\hline 137 & $19_{13,6}-18_{13,5}^{\star}$ & 104250.916 & 5 & 167 & 34.7 & 48 & Blend with $\mathrm{SO}_{2}$ and $U$-line? \\
\hline 139 & $19_{18,1}-18_{18,0}{ }^{\star}$ & 104251.566 & 5 & 278 & 6.7 & 48 & Blend with $\mathrm{SO}_{2}$ and $U$-line? \\
\hline 141 & $19_{12,7}-18_{12,6}{ }^{\star}$ & 104254.128 & 5 & 149 & 39.2 & 48 & Blend with $\mathrm{CH}_{3} \mathrm{OCHO}$ \\
\hline 143 & $19_{11,8}-18_{11,7^{\star}}^{\star}$ & 104259.358 & 5 & 132 & 43.3 & 48 & Weak, baseline problem? \\
\hline 145 & $19_{10,9}-18_{10,8}{ }^{\star}$ & 104267.390 & 5 & 117 & 47.1 & 48 & Weak \\
\hline 147 & $19_{9,10}-18_{9,9^{\star}}$ & 104279.474 & 5 & 103 & 50.6 & 48 & Blend with $\mathrm{C}_{2} \mathrm{H}_{5} \mathrm{CN}, v_{13}=1 / v_{21}=1$ and $\mathrm{C}_{2} \mathrm{H}_{5} \mathrm{OH}$ \\
\hline 149 & $19_{8,11}-18_{8,10}^{\star}$ & 104297.698 & 5 & 91 & 53.6 & 48 & Strong ${ }^{13} \mathrm{CH}_{3} \mathrm{OH}, \mathrm{CH}_{3} \mathrm{OCHO}$, and $\mathrm{CH}_{3} \mathrm{OH}$ \\
\hline 151 & $19_{7,13}-18_{7,12}^{\star}$ & 104325.811 & 5 & 80 & 56.3 & 48 & Blend with $\mathrm{C}_{2} \mathrm{H}_{3} \mathrm{CN}, v_{11}=3, \mathrm{CH}_{3}{ }^{18} \mathrm{OH}$, and $U$-lines \\
\hline 153 & $19_{6,14}-18_{6,13}^{\star}$ & 104371.094 & 5 & 71 & 58.7 & 48 & Blend with $U$-lines \\
\hline 155 & $19_{5,15}-18_{5,14}$ & 104448.349 & 5 & 63 & 60.7 & 48 & Blend with $\mathrm{C}_{2} \mathrm{H}_{5} \mathrm{CN}, v_{13}=1 / v_{21}=1$ and ${ }^{13} \mathrm{CH}_{3} \mathrm{CH}_{2} \mathrm{CN}$ \\
\hline 156 & $19_{5,14}-18_{5,13}$ & 104451.132 & 5 & 63 & 60.7 & 48 & Blend with ${ }^{13} \mathrm{CH}_{3} \mathrm{CH}_{2} \mathrm{CN}, \mathrm{C}_{2} \mathrm{H}_{3} \mathrm{CN}$, and ${ }^{13} \mathrm{CH}_{3} \mathrm{OH}$ \\
\hline 157 & $19_{3,17}-18_{3,16}$ & 104494.782 & 5 & 52 & 63.5 & 25 & Blend with $\mathrm{C}_{2} \mathrm{H}_{3} \mathrm{CN}$ and $\mathrm{C}_{2} \mathrm{H}_{3} \mathrm{CN}, v_{15}=2$ \\
\hline 158 & $19,16-184,15$ & 104567.468 & 5 & 57 & 62.3 & 25 & Blend with $\mathrm{C}_{2} \mathrm{H}_{3} \mathrm{CN}, v_{15}=1$ \\
\hline 159 & $19_{4,15}-18_{4,14}$ & 104641.877 & 5 & 57 & 62.3 & 25 & Blend with $\mathrm{C}_{2} \mathrm{H}_{3} \mathrm{CN}, v_{15}=1, \mathrm{C}_{2} \mathrm{H}_{3} \mathrm{CN}, v_{11}=1 / v_{15}=1$, and $U$-line \\
\hline 160 & $20_{1,20}-19_{1,19}$ & 105234.713 & 5 & 49 & 68.4 & 28 & Detected, blend with $\mathrm{C}_{2} \mathrm{H}_{5} \mathrm{OH}$ and $\mathrm{CH}_{3} \mathrm{OCHO}$ \\
\hline 161 & $19_{1,18}-18_{1,17}$ & 105272.047 & 5 & 47 & 64.8 & 28 & Detected, blend with $U$-lines \\
\hline 162 & $19_{3,16}-18_{3,15}$ & 105447.141 & 5 & 52 & 63.5 & 37 & Detected \\
\hline 163 & $20_{0,20}-19_{0,19}$ & 105561.113 & 5 & 49 & 68.4 & 37 & Blend with $\mathrm{CH}_{3} \mathrm{OCH}_{3}$ and $\mathrm{CH}_{3} \mathrm{CH}_{3} \mathrm{CO}$ \\
\hline 164 & $19_{2,17}-18_{2,16}$ & 106890.388 & 100 & 49 & 64.5 & 24 & Blend with $\mathrm{C}_{2} \mathrm{H}_{5} \mathrm{CN}, v_{13}=1 / v_{21}=1$ and $U$-lines \\
\hline 165 & $20_{2,19}-19_{2,18}$ & 108552.378 & 100 & 53 & 67.8 & 20 & Detected, partial blend with $\mathrm{C}_{2} \mathrm{H}_{3} \mathrm{CN}, v_{15}=1$ and $U$-lines \\
\hline 166 & $20_{16,4}-19_{16,3^{\star}}$ & 109736.466 & 5 & 234 & 24.7 & 41 & Strong $\mathrm{HC}_{3} \mathrm{~N}, v_{6}=v_{7}=1$ and $\mathrm{HCC}^{13} \mathrm{CN}, v_{5}=1 / v_{7}=3$ \\
\hline 168 & $20_{15,5}-19_{15,4}^{\star}$ & 109736.499 & 5 & 212 & 30.0 & 41 & Strong $\mathrm{HC}_{3} \mathrm{~N}, v_{6}=v_{7}=1$ and $\mathrm{HCC}^{13} \mathrm{CN}, v_{5}=1 / v_{7}=3$ \\
\hline 170 & $20_{17,3}-19_{17,2}^{\star}$ & 109737.236 & 5 & 258 & 19.0 & 41 & Strong $\mathrm{HC}_{3} \mathrm{~N}, v_{6}=v_{7}=1$ and $\mathrm{HCC}^{13} \mathrm{CN}, v_{5}=1 / v_{7}=3$ \\
\hline 172 & $20_{14,6}-19_{14,5}^{\star}$ & 109737.553 & 5 & 191 & 35.0 & 41 & Strong $\mathrm{HC}_{3} \mathrm{~N}, v_{6}=v_{7}=1$ and $\mathrm{HCC}^{13} \mathrm{CN}, v_{5}=1 / v_{7}=3$ \\
\hline 174 & $20_{18,2}-19_{18,1}^{\star}$ & 109738.645 & 5 & 283 & 13.0 & 41 & Strong $\mathrm{HC}_{3} \mathrm{~N}, v_{6}=v_{7}=1$ and $\mathrm{HCC}^{13} \mathrm{CN}, v_{5}=1 / v_{7}=3$ \\
\hline 176 & $20_{13,7}-19_{13,6}{ }^{\star}$ & 109739.922 & 5 & 172 & 39.6 & 41 & Strong $\mathrm{HC}_{3} \mathrm{~N}, v_{6}=v_{7}=1$ and $\mathrm{HCC}^{13} \mathrm{CN}, v_{5}=1 / v_{7}=3$ \\
\hline 178 & $20_{19,1}-19_{19,0}{ }^{\star}$ & 109740.558 & 6 & 310 & 6.7 & 41 & Strong $\mathrm{HC}_{3} \mathrm{~N}, v_{6}=v_{7}=1$ and $\mathrm{HCC}^{13} \mathrm{CN}, v_{5}=1 / v_{7}=3$ \\
\hline 180 & $20_{12,8}-19_{12,7^{\star}}^{\star}$ & 109744.026 & 100 & 154 & 43.9 & 41 & Strong $\mathrm{HC}_{3} \mathrm{~N}, v_{6}=v_{7}=1$ and $\mathrm{HCC}^{13} \mathrm{CN}, v_{5}=1 / v_{7}=3$ \\
\hline 182 & $20_{11,9}-19_{11,8}{ }^{\star}$ & 109750.509 & 100 & 137 & 47.9 & 41 & Strong $\mathrm{HC}_{3} \mathrm{~N}, v_{6}=v_{7}=1$ and $\mathrm{NH}_{2} \mathrm{CHO}$ \\
\hline 184 & $20_{10,10}-19_{10,9^{\star}}$ & 109760.033 & 100 & 122 & 51.4 & 41 & Strong $\mathrm{SO}_{2}$ and $\mathrm{CH}_{3} \mathrm{OCHO}, v_{\mathrm{t}}=1$ \\
\hline 186 & $20_{9,11}-19_{9,10}^{\star}$ & 109774.484 & 100 & 108 & 54.7 & 41 & Strong $\mathrm{CH}_{3} \mathrm{OCHO}, v_{\mathrm{t}}=1$ and $\mathrm{C}^{18} \mathrm{O}$ \\
\hline 188 & $20_{8,12}-19_{8,11}^{\star}$ & 109796.006 & 100 & 96 & 57.6 & 41 & Blend with $\mathrm{CH}_{3} \mathrm{CN}, v_{4}=1, \mathrm{C}^{18} \mathrm{O}$ ? and $U$-line?, uncertain baseline \\
\hline 190 & $20_{7,13}-19_{7,12}^{\star}$ & 109829.206 & 100 & 85 & 60.2 & 41 & Blend with $\mathrm{CH}_{3} \mathrm{CN}, v_{4}=1$ and $\mathrm{HNCO}$ \\
\hline 192 & $20_{6,15}-19_{6,14}$ & 109882.071 & 5 & 76 & 62.4 & 41 & Blend with $\mathrm{C}_{2} \mathrm{H}_{5} \mathrm{CN}$ \\
\hline 193 & $20_{6,14}-19_{6,13}$ & 109882.180 & 5 & 76 & 62.4 & 41 & Blend with $\mathrm{C}_{2} \mathrm{H}_{5} \mathrm{CN}$ \\
\hline 194 & $20_{5,16}-19_{5,15}$ & 109971.950 & 100 & 68 & 64.3 & 41 & Blend with $U$-lines \\
\hline
\end{tabular}


A. Belloche et al.: Detection and chemical modeling of ethyl formate and $n$-propyl cyanide in Sgr B2(N), Online Material p 3

Table 1. continued.

\begin{tabular}{|c|c|c|c|c|c|c|c|}
\hline$N^{a}$ & Transition $^{b}$ & $\begin{array}{c}\text { Frequency } \\
\text { (MHz) } \\
\text { (3) }\end{array}$ & $\begin{array}{l}\text { Unc. }^{c} \\
(\mathrm{kHz}) \\
(4)\end{array}$ & $\begin{array}{l}E_{1}^{d} \\
(\mathrm{~K}) \\
(5)\end{array}$ & $\begin{array}{l}S \mu^{2} \\
\left(\mathrm{D}^{2}\right) \\
(6)\end{array}$ & $\begin{array}{c}\sigma^{e} \\
(\mathrm{mK}) \\
(7)\end{array}$ & $\begin{array}{c}\text { Comments } \\
\text { (8) }\end{array}$ \\
\hline 195 & $20_{5,15}-19_{5,14}$ & 109976.496 & 100 & 68 & 64.3 & 41 & Blend with $U$-lines \\
\hline 196 & $20_{3,18}-19_{3,17}$ & 109982.754 & 100 & 57 & 67.0 & 41 & Blend with $U$-lines \\
\hline 197 & $20_{4,17}-19_{4,16}$ & 110103.554 & 100 & 62 & 65.8 & 24 & Blend with $\mathrm{HNCO}, v_{5}=1$ \\
\hline 198 & $20_{4,16}-19_{4,15}$ & 110209.376 & 5 & 62 & 65.8 & 24 & Blend with ${ }^{13} \mathrm{CO}$ and $\mathrm{HC}_{3} \mathrm{~N}, v_{5}=1 / v_{7}=3$ \\
\hline 199 & $21_{1,21}-20_{1,20}$ & 110417.565 & 5 & 54 & 71.8 & 24 & Blend with $\mathrm{HNCO}, v_{5}=1$ \\
\hline 200 & $20_{1,19}-19_{1,18}$ & 110544.656 & 100 & 52 & 68.2 & 32 & Blend with $\mathrm{CH}_{3}{ }^{13} \mathrm{CN}, v_{8}=1, \mathrm{C}_{2} \mathrm{H}_{5} \mathrm{OH}, \mathrm{CH}_{3} \mathrm{OCHO}$, and $U$-line \\
\hline 201 & $21_{0,21}-20_{0,20}$ & 110688.469 & 100 & 54 & 71.8 & 32 & Blend with $\mathrm{CH}_{3} \mathrm{CN}, v_{8}=1$ \\
\hline 202 & $20_{3,17}-19_{3,16}$ & 111173.805 & 100 & 57 & 67.1 & 25 & Blend with $\mathrm{CH}_{3} \mathrm{OCHO}$ \\
\hline 203 & $20_{2,18}-19_{2,17}$ & 112542.864 & 100 & 54 & 67.9 & 31 & Blend with $U$-lines \\
\hline 204 & $21_{2,20}-20_{2,19}$ & 113866.412 & 100 & 58 & 71.3 & 34 & Blend with $\mathrm{C}_{2} \mathrm{H}_{5} \mathrm{OH}$ and $U$-lines \\
\hline 205 & $21_{16,5}-20_{16,4}{ }^{\star}$ & 115224.084 & 5 & 239 & 30.2 & 60 & Strong CO \\
\hline 207 & $21_{15,6}-20_{15,5^{\star}}$ & 115224.530 & 5 & 217 & 35.3 & 60 & Strong CO \\
\hline 209 & $21_{17,4}-20_{17,3^{\star}}$ & 115224.552 & 5 & 263 & 24.8 & 60 & Strong CO \\
\hline 211 & $21_{18,3}-20_{18,2}^{\star}$ & 115225.746 & 5 & 288 & 19.1 & 60 & Strong CO \\
\hline 213 & $21_{14,7}-20_{14,6}{ }^{\star}$ & 115226.140 & 5 & 196 & 40.0 & 60 & Strong CO \\
\hline 215 & $21_{19,2}-20_{19,1}{ }^{\star}$ & 115227.514 & 6 & 315 & 13.1 & 60 & Strong CO \\
\hline 217 & $21_{13,8}-20_{13,7^{\star}}$ & 115229.252 & 5 & 177 & 44.4 & 60 & Strong CO \\
\hline 219 & $21_{20,1}-20_{20,0}$ ᄎ & 115229.733 & 7 & 343 & 6.7 & 60 & Strong CO \\
\hline 221 & $21_{12,9}-20_{12,8}{ }^{\star}$ & 115234.500 & 100 & 159 & 48.5 & 60 & Strong CO \\
\hline 223 & $21_{11,10}-20_{11,9^{\star}}$ & 115242.178 & 100 & 142 & 52.3 & 60 & Strong CO \\
\hline 225 & $21_{10,11}-20_{10,10}$ * & 115253.747 & 100 & 127 & 55.7 & 60 & Strong CO \\
\hline 227 & $21_{9,12}-20_{9,11}{ }^{\star}$ & 115270.652 & 100 & 114 & 58.8 & 60 & Strong CO \\
\hline 229 & $21_{8,13}-20_{8,12}{ }^{\star}$ & 115295.734 & 100 & 101 & 61.6 & 60 & Strong CO \\
\hline 231 & $21_{7,14}-20_{7,13}{ }^{\star}$ & 115334.205 & 100 & 91 & 64.0 & 60 & Strong CO \\
\hline 233 & $21_{6,16}-20_{6,15}$ & 115395.797 & 5 & 81 & 66.2 & 60 & Group detected, partial blend with $U$-line \\
\hline 234 & $21_{6,15}-20_{6,14}$ & 115395.986 & 5 & 81 & 66.2 & 60 & Group detected, partial blend with $U$-line \\
\hline 235 & $21_{3,19}-20_{3,18}^{\star}$ & 115462.453 & 100 & 62 & 70.5 & 60 & Weak \\
\hline 237 & $21_{5,16}-20_{5,15}$ & 115506.424 & 100 & 73 & 68.0 & 60 & Weak \\
\hline 238 & $22_{1,22}-21_{1,21}$ & 115595.764 & 5 & 59 & 75.2 & 79 & Detected, blend with $\mathrm{CH}_{3} \mathrm{CH}_{3} \mathrm{CO}, v_{t}=1$, uncertain baseline \\
\hline 239 & $21_{4,18}-20_{4,17}$ & 115641.781 & 100 & 67 & 69.4 & 79 & Blend with $U$-line \\
\hline 240 & $21_{1,20}-20_{1,19}$ & 115778.715 & 100 & 57 & 71.6 & 79 & Blend with $\mathrm{CH}_{3} \mathrm{OCH}_{3}$ \\
\hline 241 & $21_{4,17}-20_{4,16}$ & 115789.775 & 5 & 67 & 69.4 & 79 & Weak \\
\hline 242 & $22_{0,22}-21_{0,21}$ & 115819.018 & 100 & 59 & 75.2 & 79 & Weak \\
\hline 243 & $26_{1,26}-25_{1,25}$ & 136272.994 & 100 & 83 & 88.9 & 28 & Blend with $\mathrm{HC}_{3} \mathrm{~N}, v_{4}=1$ and $\mathrm{CH}_{3} \mathrm{OCHO}$ \\
\hline 244 & $26_{0,26}-25_{0,25}$ & 136369.733 & 100 & 83 & 88.9 & 28 & Blend with $U$-line \\
\hline 245 & $25_{1,24}-24_{1,23}$ & 136400.164 & 100 & 81 & 85.2 & 28 & Blend with $\mathrm{HC}^{13} \mathrm{CCN}, v_{7}=1$ and $\mathrm{C}_{2} \mathrm{H}_{3} \mathrm{CN}, v_{11}=2$ \\
\hline 246 & $27_{2,26}-26_{2,25}$ & 145476.697 & 100 & 94 & 91.9 & 25 & Blend with $\mathrm{C}_{2} \mathrm{H}_{5} \mathrm{CN}, v_{13}=1 / v_{21}=1$ and $\mathrm{O}^{13} \mathrm{CS}$ \\
\hline 247 & $28_{5,24}-27_{5,23}$ & 154303.763 & 50 & 118 & 93.0 & 112 & Blend with $\mathrm{C}_{2} \mathrm{H}_{5} \mathrm{OH}$ and $\mathrm{CH}_{3} \mathrm{OCH}_{3}$ \\
\hline 248 & $28_{5,23}-27_{5,22}$ & 154393.012 & 50 & 118 & 93.0 & 112 & Blend with $\mathrm{HCC}^{13} \mathrm{CN}, v_{7}=1$ \\
\hline 249 & $28_{4,25}-27_{4,24}$ & 154410.681 & 50 & 111 & 94.1 & 112 & Strong $\mathrm{HNCO}$ and $\mathrm{CH}_{3} \mathrm{OH}$ \\
\hline 250 & $31_{1,30}-30_{2,29}$ & 163397.978 & 100 & 124 & 9.6 & 38 & Blend with $\mathrm{C}_{2} \mathrm{H}_{5} \mathrm{CN}$ and $\mathrm{CH}_{3} \mathrm{COOH}$ \\
\hline 251 & $31_{2,30}-30_{2,29}$ & 166330.213 & 100 & 124 & 105.7 & 66 & Blend with ${ }^{13} \mathrm{CH}_{3} \mathrm{CH}_{2} \mathrm{CN}$ and $U$-lines \\
\hline 252 & $33_{1,33}-32_{0,32}$ & 172468.973 & 100 & 134 & 14.3 & 44 & Strong $\mathrm{C}_{2} \mathrm{H}_{5} \mathrm{CN}$ and $\mathrm{CH}_{3} \mathrm{OCHO}, v_{\mathrm{t}}=1$ \\
\hline 253 & $31_{4,27}-30_{4,26}$ & 172707.869 & 100 & 135 & 104.6 & 44 & Blend with $\mathrm{H}^{13} \mathrm{CN}$ in absorption and $\mathrm{C}_{2} \mathrm{H}_{5} \mathrm{CN}, v_{13}=1 / v_{21}=1$ \\
\hline 254 & $32_{7,26}-31_{7,25}$ & 176045.797 & 4 & 166 & 104.5 & 365 & Noisy \\
\hline 255 & $32_{7,25}-31_{7,24}$ & 176046.676 & 4 & 166 & 104.5 & 365 & Noisy \\
\hline 256 & $32_{6,27}-31_{6,26}$ & 176266.224 & 100 & 157 & 105.9 & 365 & Noisy \\
\hline 257 & $32_{6,26}-31_{6,25}$ & 176285.887 & 100 & 157 & 105.9 & 365 & Noisy \\
\hline 258 & $38_{1,37}-37_{2,36}$ & 201872.699 & 100 & 185 & 13.2 & 138 & Strong ${ }^{13} \mathrm{CH}_{3} \mathrm{CH}_{2} \mathrm{CN}$ \\
\hline 259 & $53_{2,51}-53_{1,52}$ & 201925.976 & 100 & 370 & 6.7 & 138 & Strong $\mathrm{CH}_{2} \mathrm{CO}$ \\
\hline 260 & $36_{4,32}-35_{4,31}$ & 201931.928 & 100 & 179 & 122.0 & 138 & Strong $\mathrm{CH}_{2} \mathrm{CO}$ \\
\hline 261 & $36_{3,33}-35_{3,32}$ & 202388.460 & 100 & 175 & 122.6 & 108 & Blend with $\mathrm{C}_{2} \mathrm{H}_{3} \mathrm{CN}, v_{11}=1$ \\
\hline 262 & $53_{3,51}-53_{2,52}$ & 202394.525 & 28 & 370 & 6.7 & 108 & Blend with $\mathrm{C}_{2} \mathrm{H}_{3} \mathrm{CN}, v_{11}=1$ \\
\hline 263 & $62_{5,58}-62_{3,59}$ & 202561.279 & 17 & 524 & 1.4 & 108 & Strong $\mathrm{C}_{2} \mathrm{H}_{3} \mathrm{CN}, v_{15}=1$ \\
\hline 264 & $38_{2,37}-37_{2,36}$ & 202563.884 & 100 & 185 & 129.7 & 108 & Strong $\mathrm{C}_{2} \mathrm{H}_{3} \mathrm{CN}, v_{15}=1$ \\
\hline 265 & $38_{1,37}-37_{1,36}$ & 202730.394 & 100 & 185 & 129.7 & 108 & Blend with $\mathrm{CH}_{3} \mathrm{OCH}_{3}$ and $\mathrm{C}_{2} \mathrm{H}_{3} \mathrm{CN}, v_{15}=1$ \\
\hline 266 & $37_{23,14}-36_{23,13}^{\star}$ & 203008.778 & 28 & 556 & 77.9 & 138 & Blend with $U$-line \\
\hline 268 & $37_{22,15}-36_{22,14^{\star}}^{\star}$ & 203008.823 & 19 & 523 & 82.0 & 138 & Blend with $U$-line \\
\hline 270 & $37_{24,13}-36_{24,12}{ }^{\star}$ & 203009.542 & 40 & 589 & 73.5 & 138 & Blend with $U$-line \\
\hline 272 & $37_{21,16}-36_{21,15}{ }^{\star}$ & 203009.945 & 13 & 492 & 86.0 & 138 & Blend with $U$-line \\
\hline 274 & $37_{25,12}-36_{25,11}{ }^{\star}$ & 203010.874 & 56 & 624 & 69.0 & 138 & Blend with $U$-line \\
\hline 276 & $37_{20,17}-36_{20,16^{\star}}$ & 203012.456 & 9 & 463 & 89.8 & 138 & Blend with $U$-line \\
\hline 278 & $37_{26,11}-36_{26,10^{\star}}$ & 203012.555 & 77 & 661 & 64.2 & 138 & Blend with $U$-line \\
\hline 280 & $37_{27,10}-36_{27,9^{\star}}$ & 203014.377 & 104 & 699 & 59.3 & 138 & Blend with $U$-line \\
\hline
\end{tabular}


Table 1. continued.

\begin{tabular}{|c|c|c|c|c|c|c|c|}
\hline$N^{a}$ & Transition $^{b}$ & $\begin{array}{c}\text { Frequency } \\
\text { (MHz) } \\
\text { (3) }\end{array}$ & $\begin{array}{l}\text { Unc. }^{c} \\
(\mathrm{kHz}) \\
(4)\end{array}$ & $\begin{array}{l}E_{1}^{d} \\
(\mathrm{~K}) \\
(5)\end{array}$ & $\begin{array}{l}S \mu^{2} \\
\left(D^{2}\right) \\
(6)\end{array}$ & $\begin{array}{c}\sigma^{e} \\
(\mathrm{mK}) \\
(7)\end{array}$ & $\begin{array}{c}\text { Comments } \\
\text { (8) }\end{array}$ \\
\hline 282 & $37_{28,9}-36_{28,8}{ }^{\star}$ & 203016.140 & 138 & 738 & 54.2 & 138 & Blend with $U$-line \\
\hline 284 & $37_{19,18}-36_{19,17^{\star}}$ & 203016.766 & 100 & 435 & 93.4 & 138 & Blend with $U$-line \\
\hline 286 & $37_{29,8}-36_{29,7}^{\star}$ & 203017.650 & 181 & 779 & 48.9 & 138 & Blend with $U$-line \\
\hline 288 & $37_{18,19}-36_{18,18}{ }^{\star}$ & 203023.193 & 6 & 408 & 96.9 & 138 & Blend with $\mathrm{CH}_{3} \mathrm{OCH}_{3}$ \\
\hline 290 & $37_{17,20}-36_{17,19^{\star}}$ & 203032.356 & 100 & 383 & 100.1 & 138 & Blend with $\mathrm{CH}_{3} \mathrm{OCH}_{3}$ and $\mathrm{CH}_{3}{ }^{13} \mathrm{CN}, v_{8}=1$ \\
\hline 292 & $37_{16,21}-36_{16,20} \star$ & 203045.040 & 100 & 360 & 103.2 & 138 & Blend with $\mathrm{CH}_{3}{ }^{13} \mathrm{CH}_{2} \mathrm{CN}$ and $U$-line \\
\hline 294 & $37_{15,22}-36_{15,21}{ }^{\star}$ & 203062.173 & 100 & 337 & 106.1 & 138 & Blend with ${ }^{13} \mathrm{CH}_{3} \mathrm{CH}_{2} \mathrm{CN}$ \\
\hline 296 & $37_{14,23}-36_{14,22}{ }^{\star}$ & 203085.040 & 100 & 316 & 108.7 & 138 & Blend with $\mathrm{CH}_{3}{ }^{13} \mathrm{CH}_{2} \mathrm{CN}$ \\
\hline 298 & $37_{2,35}-36_{2,34}$ & 203093.256 & 100 & 181 & 126.0 & 138 & Blend with $\mathrm{H}^{13} \mathrm{CCCN}, v_{6}=1$ \\
\hline 299 & $37_{13,24}-36_{13,23}{ }^{\star}$ & 203115.417 & 100 & 297 & 111.2 & 138 & Blend with $\mathrm{C}_{2} \mathrm{H}_{3} \mathrm{CN}, v_{11}=1 / v_{15}=1$ \\
\hline 301 & $37_{12,25}-36_{12,24}{ }^{\star}$ & 203155.887 & 100 & 279 & 113.6 & 138 & Strong $\mathrm{CH}_{3} \mathrm{CN}, v_{8}=1$ \\
\hline 303 & $37_{11,26}-36_{11,25}{ }^{\star}$ & 203210.265 & 100 & 263 & 115.7 & 138 & Blend with $U$-line \\
\hline 305 & $39_{0,39}-38_{1,38}$ & 203277.722 & 100 & 187 & 17.2 & 161 & Blend with $\mathrm{C}_{2} \mathrm{H}_{3} \mathrm{CN}, v_{11}=1$ \\
\hline 306 & $37_{10,27}-36_{10,26^{\star}}$ & 203284.465 & 100 & 247 & 117.6 & 161 & Blend with $\mathrm{C}_{2} \mathrm{H}_{3} \mathrm{CN}, v_{11}=1$ and $U$-line \\
\hline 308 & $39_{1,39}-38_{1,38}$ & 203293.314 & 100 & 187 & 133.5 & 161 & Blend with $\mathrm{CH}_{3} \mathrm{CN}, v_{8}=2$ \\
\hline 309 & $39_{0,39}-38_{0,38}$ & 203297.717 & 100 & 187 & 133.5 & 161 & Blend with $\mathrm{CH}_{3} \mathrm{CN}, v_{8}=2$ \\
\hline 310 & $39_{1,39}-38_{0,38}$ & 203313.274 & 100 & 187 & 17.2 & 161 & Blend with $U$-line \\
\hline 311 & $37_{9,29}-36_{9,28}^{\star}$ & 203387.942 & 4 & 234 & 119.4 & 161 & Strong $\mathrm{C}_{2} \mathrm{H}_{5} \mathrm{CN}, \mathrm{CH}_{3} \mathrm{OCH}_{3}$, and $\mathrm{SO}_{2}$ \\
\hline 313 & $7_{6,2}-6_{5,1}{ }^{\star}$ & 203409.735 & 13 & 24 & 2.7 & 161 & Strong $\mathrm{C}_{2} \mathrm{H}_{5} \mathrm{CN}, \mathrm{CH}_{3} \mathrm{OCH}_{3}$, and ${ }^{13} \mathrm{CH}_{2} \mathrm{CHCN}$ \\
\hline 315 & $38_{2,37}-37_{1,36}$ & 203421.857 & 4 & 185 & 13.2 & 161 & Strong $\mathrm{CH}_{3} \mathrm{OCH}_{3}$ and ${ }^{13} \mathrm{CH}_{2} \mathrm{CHCN}$ \\
\hline 316 & $37_{8,30}-36_{8,29}$ & 203536.955 & 4 & 222 & 121.0 & 161 & Blend with $\mathrm{CH}_{3} \mathrm{CN}, v_{8}=2$ and ${ }^{13} \mathrm{CH}_{2} \mathrm{CHCN}$ \\
\hline 317 & $37_{8,29}-36_{8,28}$ & 203537.200 & 4 & 222 & 121.0 & 161 & Blend with $\mathrm{CH}_{3} \mathrm{CN}, v_{8}=2$ and ${ }^{13} \mathrm{CH}_{2} \mathrm{CHCN}$ \\
\hline 318 & $37_{7,31}-36_{7,30}$ & 203760.083 & 100 & 211 & 122.4 & 364 & Blend with $U$-line \\
\hline 319 & $37_{7,30}-36_{7,29}$ & 203765.767 & 100 & 211 & 122.4 & 364 & Blend with $\mathrm{C}_{3} \mathrm{H}_{7} \mathrm{CN}$ and ${ }^{13} \mathrm{CH}_{2} \mathrm{CHCN}$ \\
\hline 320 & $37_{4,34}-36_{4,33}$ & 203781.525 & 4 & 188 & 125.4 & 364 & Blend with ${ }^{13} \mathrm{CH}_{2} \mathrm{CHCN}$ and $U$-line \\
\hline 321 & $18_{4,14}-17_{3,15}$ & 203899.881 & 11 & 47 & 2.8 & 364 & Blend with $\mathrm{C}_{3} \mathrm{H}_{7} \mathrm{CN}$ and $U$-line \\
\hline 322 & $37_{6,32}-36_{6,31}$ & 204086.275 & 100 & 202 & 123.6 & 364 & Blend with $\mathrm{H}^{13} \mathrm{CCCN}, v_{7}=2$ and $U$-line \\
\hline 323 & $34_{3,32}-33_{2,31}$ & 204129.239 & 11 & 153 & 7.0 & 316 & Blend with ${ }^{34} \mathrm{SO}_{2}$ \\
\hline 324 & $37_{6,31}-36_{6,30}$ & 204178.927 & 100 & 202 & 123.6 & 316 & Blend with $\mathrm{CH}_{3} \mathrm{CH}_{3} \mathrm{CO}$ and $U$-line? \\
\hline 325 & $37_{5,33}-36_{5,32}$ & 204364.928 & 4 & 194 & 124.6 & 316 & Blend with $\mathrm{CH}_{3} \mathrm{CH}_{3} \mathrm{CO}$ \\
\hline 326 & $82_{8,75}-81_{9,72}$ & 204369.553 & 82 & 938 & 5.1 & 316 & Blend with $\mathrm{CH}_{3} \mathrm{CH}_{3} \mathrm{CO}$ and $\mathrm{SO}_{2}$ \\
\hline 327 & $75_{8,67}-74_{9,66}$ & 205281.393 & 47 & 792 & 5.8 & 100 & Blend with $\mathrm{SO}_{2}$ \\
\hline 328 & $37_{5,32}-36_{5,31}$ & 205282.240 & 100 & 194 & 124.6 & 100 & Blend with $\mathrm{SO}_{2}$ \\
\hline 329 & $13_{5,9}-12_{4,8}$ & 206264.566 & 12 & 32 & 2.8 & 280 & Strong $\mathrm{C}_{2} \mathrm{H}_{5} \mathrm{CN}$ and $\mathrm{CH}_{3} \mathrm{OCHO}$ \\
\hline 330 & $13_{5,8}-12_{4,9}$ & 206270.541 & 12 & 32 & 2.8 & 280 & Strong $\mathrm{C}_{2} \mathrm{H}_{5} \mathrm{CN}$ and $\mathrm{CH}_{3} \mathrm{OCHO}$ \\
\hline 331 & $38_{3,36}-37_{3,35}$ & 206596.942 & 100 & 191 & 129.3 & 106 & Strong $\mathrm{C}_{2} \mathrm{H}_{5} \mathrm{CN}, v_{13}=1 / v_{21}=1$ \\
\hline 332 & $19_{4,16}-18_{3,15}$ & 206607.866 & 11 & 52 & 2.9 & 106 & Strong $\mathrm{C}_{2} \mathrm{H}_{5} \mathrm{CN}$ \\
\hline 333 & $35_{3,33}-34_{2,32}$ & 207085.257 & 11 & 162 & 7.5 & 117 & Strong $\mathrm{C}_{2} \mathrm{H}_{5} \mathrm{CN}$ \\
\hline 334 & $39_{1,38}-38_{2,37}$ & 207168.207 & 4 & 195 & 13.7 & 117 & Blend with $\mathrm{CH}_{3} \mathrm{CH}_{3} \mathrm{CO}$ and $U$-line \\
\hline 335 & $39_{2,38}-38_{2,37}$ & 207723.987 & 100 & 195 & 133.1 & 282 & Blend with $U$-line and $\mathrm{C}_{2} \mathrm{H}_{5} \mathrm{CN}, v_{13}=1 / v_{21}=1$ \\
\hline 336 & $37_{4,33}-36_{4,32}$ & 207774.900 & 4 & 189 & 125.5 & 282 & Blend with $\mathrm{C}_{2} \mathrm{H}_{5} \mathrm{OH}$ \\
\hline 337 & $37_{3,34}-36_{3,33}$ & 207820.202 & 100 & 185 & 126.0 & 173 & Blend with $\mathrm{C}_{3} \mathrm{H}_{7} \mathrm{CN}$ and $\mathrm{C}_{2} \mathrm{H}_{5} \mathrm{OH}$ \\
\hline 338 & $39_{1,38}-38_{1,37}$ & 207859.555 & 4 & 195 & 133.1 & 173 & Blend with $\mathrm{C}_{2} \mathrm{H}_{5} \mathrm{CN}, v_{13}=1 / v_{21}=1$ \\
\hline 339 & $38_{2,36}-37_{2,35}$ & 208144.350 & 100 & 191 & 129.4 & 173 & Blend with $\mathrm{CH}_{2} \mathrm{CH}^{13} \mathrm{CN}$ and $\mathrm{C}_{2} \mathrm{H}_{3} \mathrm{CN}, v_{11}=1$ \\
\hline 340 & $39_{2,38}-38_{1,37}$ & 208415.332 & 4 & 195 & 13.7 & 168 & Strong $\mathrm{C}_{2} \mathrm{H}_{5} \mathrm{CN}$ \\
\hline 341 & $40_{0,40}-39_{1,39}$ & 208431.560 & 5 & 197 & 17.7 & 168 & Strong $\mathrm{C}_{2} \mathrm{H}_{5} \mathrm{CN}$ \\
\hline 342 & $40_{1,40}-39_{1,39}$ & 208443.521 & 100 & 197 & 137.0 & 168 & Strong $\mathrm{C}_{2} \mathrm{H}_{5} \mathrm{CN}$ \\
\hline 343 & $40_{0,40}-39_{0,39}$ & 208447.014 & 100 & 197 & 137.0 & 168 & Strong $\mathrm{C}_{2} \mathrm{H}_{5} \mathrm{CN}$ \\
\hline 344 & $40_{1,40}-39_{0,39}$ & 208459.081 & 5 & 197 & 17.7 & 168 & Strong $\mathrm{C}_{2} \mathrm{H}_{5} \mathrm{CN}$ \\
\hline 345 & $38_{23,15}-37_{23,14}{ }^{\star}$ & 208493.011 & 28 & 565 & 82.6 & 168 & Strong $\mathrm{C}_{2} \mathrm{H}_{5} \mathrm{CN}$ \\
\hline 347 & $38_{24,14}-37_{24,13}{ }^{\star}$ & 208493.410 & 41 & 599 & 78.3 & 168 & Strong $\mathrm{C}_{2} \mathrm{H}_{5} \mathrm{CN}$ \\
\hline 349 & $38_{22,16}-37_{22,15^{\star}}$ & 208493.496 & 19 & 533 & 86.6 & 168 & Strong $\mathrm{C}_{2} \mathrm{H}_{5} \mathrm{CN}$ \\
\hline 351 & $38_{25,13}-37_{25,12}{ }^{\star}$ & 208494.438 & 57 & 634 & 73.9 & 168 & Strong $\mathrm{C}_{2} \mathrm{H}_{5} \mathrm{CN}$ \\
\hline 353 & $38_{21,17}-37_{21,16}{ }^{\star}$ & 208495.153 & 13 & 502 & 90.5 & 168 & Strong $\mathrm{C}_{2} \mathrm{H}_{5} \mathrm{CN}$ \\
\hline 355 & $38_{26,12}-37_{26,11}{ }^{\star}$ & 208495.863 & 79 & 671 & 69.3 & 168 & Strong $\mathrm{C}_{2} \mathrm{H}_{5} \mathrm{CN}$ \\
\hline 357 & $38_{27,11}-37_{27,10^{\star}}$ & 208497.466 & 107 & 709 & 64.5 & 168 & Strong $\mathrm{C}_{2} \mathrm{H}_{5} \mathrm{CN}$ \\
\hline 359 & $38_{20,18}-37_{20,17^{\star}}$ & 208498.313 & 9 & 473 & 94.2 & 168 & Strong $\mathrm{C}_{2} \mathrm{H}_{5} \mathrm{CN}$ \\
\hline 361 & $38_{28,10}-37_{28,9^{\star}}$ & 208499.037 & 142 & 748 & 59.6 & 168 & Strong $\mathrm{C}_{2} \mathrm{H}_{5} \mathrm{CN}$ \\
\hline 363 & $38_{29,9}-37_{29,8}{ }^{\star}$ & 208500.372 & 185 & 789 & 54.4 & 168 & Strong $\mathrm{C}_{2} \mathrm{H}_{5} \mathrm{CN}$ \\
\hline 365 & $38_{19,19}-37_{19,18^{\star}}$ & 208503.415 & 100 & 445 & 97.8 & 168 & Strong $\mathrm{C}_{2} \mathrm{H}_{5} \mathrm{CN}, v_{13}=1 / v_{21}=1$ \\
\hline 367 & $38_{18,20}-37_{18,19}{ }^{\star}$ & 208510.857 & 100 & 418 & 101.1 & 168 & Blend with $\mathrm{C}_{2} \mathrm{H}_{5} \mathrm{OH}$ and $\mathrm{C}_{2} \mathrm{H}_{3} \mathrm{CN}$ \\
\hline 369 & $38_{17,21}-37_{17,20}$ * & 208521.187 & 100 & 393 & 104.3 & 168 & Blend with $\mathrm{C}_{2} \mathrm{H}_{5} \mathrm{OH}$ \\
\hline 371 & $38_{16,22}-37_{16,21}{ }^{\star}$ & 208535.402 & 100 & 369 & 107.2 & 168 & Blend with $\mathrm{C}_{2} \mathrm{H}_{5} \mathrm{OH}$ \\
\hline 373 & $38_{15,23}-37_{15,22}{ }^{\star}$ & 208554.387 & 100 & 347 & 110.0 & 168 & Blend with $\mathrm{C}_{2} \mathrm{H}_{5} \mathrm{CN}, v_{13}=1 / v_{21}=1$ and $U$-line \\
\hline
\end{tabular}


Table 1. continued.

\begin{tabular}{|c|c|c|c|c|c|c|c|}
\hline$N^{a}$ & Transition $^{b}$ & $\begin{array}{c}\text { Frequency } \\
(\mathrm{MHz}) \\
\text { (3) }\end{array}$ & $\begin{array}{l}\text { Unc. }^{c} \\
\text { (kHz) } \\
(4)\end{array}$ & $\begin{array}{l}E_{1}^{d} \\
(\mathrm{~K}) \\
(5)\end{array}$ & $\begin{array}{l}S \mu^{2} \\
\left(D^{2}\right) \\
(6)\end{array}$ & $\begin{array}{c}\sigma^{e} \\
(\mathrm{mK}) \\
(7)\end{array}$ & Comments \\
\hline 375 & $38_{14,24}-37_{14,23}{ }^{\star}$ & 208579.484 & 100 & 326 & 112.6 & 168 & Blend with $\mathrm{CH}_{3} \mathrm{CH}_{3} \mathrm{CO}, v_{t}=1$ and $U$-line \\
\hline 377 & $38_{13,25}-37_{13,24}{ }^{\star}$ & 208612.841 & 4 & 307 & 115.1 & 168 & Strong $\mathrm{C}_{2} \mathrm{H}_{5} \mathrm{CN}, v_{13}=1 / v_{21}=1$ \\
\hline 379 & $38_{12,26}-37_{12,25}{ }^{\star}$ & 208657.029 & 4 & 289 & 117.3 & 168 & Blend with $\mathrm{CH}_{3} \mathrm{OCHO}$ and $\mathrm{CH}_{3} \mathrm{CH}_{3} \mathrm{CO}$ \\
\hline 381 & $38_{11,27}-37_{11,26}{ }^{\star}$ & 208716.183 & 100 & 272 & 119.4 & 160 & Blend with $\mathrm{CH}_{3} \mathrm{OCHO}$ \\
\hline 383 & $38_{10,29}-37_{10,28}{ }^{\star}$ & 208796.910 & 4 & 257 & 121.3 & 160 & Blend with $\mathrm{CH}_{2} \mathrm{CH}^{13} \mathrm{CN}$ and $\mathrm{CH}_{3} \mathrm{OCHO}$ \\
\hline 385 & $8_{6,3}-7_{5,2}{ }^{\star}$ & 208895.157 & 13 & 25 & 2.8 & 160 & Strong $\mathrm{CH}_{3} \mathrm{OCHO}$ and $\mathrm{C}_{2} \mathrm{H}_{3} \mathrm{CN}$ \\
\hline 387 & $38_{8,31}-37_{8,30}$ & 209071.357 & 4 & 231 & 124.6 & 160 & Strong $\mathrm{C}_{2} \mathrm{H}_{3} \mathrm{CN}$ \\
\hline 388 & $38_{8,30}-37_{8,29}$ & 209071.723 & 4 & 231 & 124.6 & 160 & Strong $\mathrm{C}_{2} \mathrm{H}_{3} \mathrm{CN}$ \\
\hline 389 & $38_{4,35}-37_{4,34}$ & 209201.665 & 4 & 197 & 128.8 & 58 & Strong $\mathrm{H}_{2} \mathrm{CS}$ and $\mathrm{C}_{2} \mathrm{H}_{3} \mathrm{CN}, v_{15}=1$ \\
\hline 390 & $38_{7,32}-37_{7,31}$ & 209313.439 & 4 & 221 & 125.9 & 58 & Strong $\mathrm{C}_{2} \mathrm{H}_{3} \mathrm{CN}, v_{15}=1$ \\
\hline 391 & $38_{7,31}-37_{7,30}$ & 209321.499 & 4 & 221 & 125.9 & 58 & Strong $\mathrm{C}_{2} \mathrm{H}_{3} \mathrm{CN}, v_{15}=1$ \\
\hline 392 & $19_{4,15}-18_{3,16}$ & 209541.953 & 11 & 52 & 2.9 & 58 & Blend with $\mathrm{C}_{2} \mathrm{H}_{3} \mathrm{CN}$ \\
\hline 393 & $38_{6,33}-37_{6,32}$ & 209660.931 & 4 & 212 & 127.1 & 45 & $\begin{array}{l}\text { Blend with } \mathrm{C}_{2} \mathrm{H}_{3} \mathrm{CN}, v_{11}=1, \mathrm{HC}^{13} \mathrm{CCN}, v_{7}=2 \text {, and } \\
\mathrm{HCC}^{13} \mathrm{CN}, v_{7}=2\end{array}$ \\
\hline 394 & $38_{6,32}-37_{6,31}$ & 209783.452 & 100 & 212 & 127.1 & 45 & Blend with $\mathrm{C}_{2} \mathrm{H}_{3} \mathrm{CN}, v_{11}=1 / v_{15}=1$ \\
\hline 395 & $38_{5,34}-37_{5,33}$ & 209918.890 & 4 & 204 & 128.1 & 45 & Strong $\mathrm{CH}_{3} \mathrm{OCHO}$ \\
\hline 396 & $36_{3,34}-35_{2,33}$ & 210218.541 & 10 & 172 & 8.0 & 64 & Blend with $\mathrm{C}_{2} \mathrm{H}_{3} \mathrm{CN}, v_{11}=2$ \\
\hline 397 & $40_{2,38}-39_{3,37}$ & 210705.222 & 6 & 211 & 10.0 & 37 & Blend with $\mathrm{C}_{2} \mathrm{H}_{3} \mathrm{CN}, v_{11}=2$ and $U$-line \\
\hline 398 & $38_{5,33}-37_{5,32}$ & 211044.215 & 100 & 204 & 128.1 & 33 & Blend with $\mathrm{C}_{2} \mathrm{H}_{5} \mathrm{CN}$ \\
\hline 399 & $20_{4,17}-19_{3,16}$ & 211264.158 & 11 & 57 & 3.0 & 33 & Strong $\mathrm{CH}_{3} \mathrm{OCHO}$ \\
\hline 400 & $14_{5,10}-13_{4,9}$ & 211717.651 & 12 & 35 & 2.9 & 47 & Strong $\mathrm{C}_{2} \mathrm{H}_{3} \mathrm{CN}$ \\
\hline 401 & $14_{5,9}-13_{4,10}$ & 211728.949 & 12 & 35 & 2.9 & 47 & Strong $\mathrm{C}_{2} \mathrm{H}_{3} \mathrm{CN}$ \\
\hline 402 & $39_{3,37}-38_{3,36}$ & 211830.506 & 100 & 201 & 132.7 & 47 & Blend with $\mathrm{NH}_{2}{ }^{13} \mathrm{CHO}, v_{12}=1$ \\
\hline 403 & $51_{1,50}-51_{0,51}$ & 211833.125 & 40 & 333 & 3.3 & 47 & Blend with $\mathrm{NH}_{2}{ }^{13} \mathrm{CHO}, v_{12}=1$ \\
\hline 404 & $40_{1,39}-39_{2,38}$ & 212435.627 & 4 & 205 & 14.2 & 36 & Strong $\mathrm{NH}_{2} \mathrm{CHO}$ and $\mathrm{CH}_{3} \mathrm{OCHO}$ \\
\hline 405 & $40_{2,39}-39_{2,38}$ & 212881.373 & 100 & 205 & 136.5 & 99 & Blend with $\mathrm{H}^{13} \mathrm{CCCN}, v_{7}=2$ \\
\hline 406 & $40_{1,39}-39_{1,38}$ & 212991.436 & 100 & 205 & 136.5 & 99 & Blend with ${ }^{34} \mathrm{SO}_{2}$ and $U$-line? \\
\hline 407 & $39_{2,37}-38_{2,36}$ & 213191.655 & 100 & 201 & 132.8 & 48 & Blend with $\mathrm{CH}_{2}(\mathrm{OH}) \mathrm{CHO}$ \\
\hline 408 & $38_{3,35}-37_{3,34}$ & 213203.256 & 100 & 195 & 129.4 & 48 & Blend with $\mathrm{CH}_{2}(\mathrm{OH}) \mathrm{CHO}$ \\
\hline 409 & $40_{2,39}-39_{1,38}$ & 213437.154 & 5 & 205 & 14.2 & 48 & Blend with $\mathrm{C}_{2} \mathrm{H}_{5} \mathrm{OH}$ and $\mathrm{CH}_{3} \mathrm{OCH}_{3}$ \\
\hline 410 & $37_{3,35}-36_{2,34}$ & 213536.157 & 9 & 181 & 8.5 & 48 & Blend with $\mathrm{C}_{2} \mathrm{H}_{3} \mathrm{CN}, v_{11}=2$ \\
\hline 411 & $41_{0,41}-40_{1,40}$ & 213584.046 & 5 & 207 & 18.2 & 48 & Blend with ${ }^{13} \mathrm{CH}_{2} \mathrm{CHCN}$ \\
\hline 412 & $41_{1,41}-40_{1,40}$ & 213593.386 & 100 & 207 & 140.4 & 48 & Partial blend with $\mathrm{H}^{13} \mathrm{CCCN}, v_{5}=1 / v_{7}=3$, uncertain baseline \\
\hline 413 & $41_{0,41}-40_{0,40}$ & 213596.056 & 100 & 207 & 140.4 & 48 & Partial blend with $\mathrm{H}^{13} \mathrm{CCCN}, v_{5}=1 / v_{7}=3$, uncertain baseline \\
\hline 414 & $38_{4,34}-37_{4,33}$ & 213600.651 & 100 & 199 & 129.0 & 48 & Partial blend with $\mathrm{H}^{13} \mathrm{CCCN}, v_{5}=1 / v_{7}=3$, uncertain baseline \\
\hline 415 & $41_{1,41}-40_{0,40}$ & 213605.377 & 5 & 207 & 18.2 & 48 & Partial blend with $\mathrm{H}^{13} \mathrm{CCCN}, v_{5}=1 / v_{7}=3$, uncertain baseline \\
\hline 416 & $39_{23,16}-38_{23,15}^{\star}$ & 213977.041 & 29 & 575 & 87.2 & 48 & Strong $\mathrm{C}_{2} \mathrm{H}_{5}{ }^{13} \mathrm{CN}$ and $\mathrm{CH}_{3}{ }^{13} \mathrm{CH}_{2} \mathrm{CN}$ \\
\hline 418 & $39_{24,15}-38_{24,14}{ }^{\star}$ & 213977.045 & 42 & 609 & 83.1 & 48 & Strong $\mathrm{C}_{2} \mathrm{H}_{5}{ }^{13} \mathrm{CN}$ and $\mathrm{CH}_{3}{ }^{13} \mathrm{CH}_{2} \mathrm{CN}$ \\
\hline 420 & $39_{25,14}-38_{25,13}{ }^{\star}$ & 213977.743 & 59 & 644 & 78.8 & 48 & Strong $\mathrm{C}_{2} \mathrm{H}_{5}{ }^{13} \mathrm{CN}$ and $\mathrm{CH}_{3}{ }^{13} \mathrm{CH}_{2} \mathrm{CN}$ \\
\hline 422 & $39_{22,17}-38_{22,16^{\star}}$ & 213978.002 & 20 & 543 & 91.2 & 48 & Strong $\mathrm{C}_{2} \mathrm{H}_{5}{ }^{13} \mathrm{CN}$ and $\mathrm{CH}_{3}{ }^{13} \mathrm{CH}_{2} \mathrm{CN}$ \\
\hline 424 & $39_{26,13}-38_{26,12}{ }^{\star}$ & 213978.888 & 81 & 681 & 74.3 & 48 & Strong $\mathrm{C}_{2} \mathrm{H}_{5}{ }^{13} \mathrm{CN}$ and $\mathrm{CH}_{3}{ }^{13} \mathrm{CH}_{2} \mathrm{CN}$ \\
\hline 426 & $39_{21,18}-38_{21,17^{\star}}$ & 213980.233 & 13 & 512 & 95.0 & 48 & Strong $\mathrm{C}_{2} \mathrm{H}_{5}{ }^{13} \mathrm{CN}$ and $\mathrm{CH}_{3}{ }^{13} \mathrm{CH}_{2} \mathrm{CN}$ \\
\hline 428 & $39_{27,12}-38_{27,11}{ }^{\star}$ & 213980.250 & 109 & 719 & 69.7 & 48 & Strong $\mathrm{C}_{2} \mathrm{H}_{5}{ }^{13} \mathrm{CN}$ and $\mathrm{CH}_{3}{ }^{13} \mathrm{CH}_{2} \mathrm{CN}$ \\
\hline 430 & $39_{28,11}-38_{28,10}{ }^{\star}$ & 213981.610 & 145 & 758 & 64.8 & 48 & Strong $\mathrm{C}_{2} \mathrm{H}_{5}{ }^{13} \mathrm{CN}$ and $\mathrm{CH}_{3}{ }^{13} \mathrm{CH}_{2} \mathrm{CN}$ \\
\hline 432 & $39_{29,10}-38_{29,9}{ }^{\star}$ & 213982.754 & 190 & 799 & 59.8 & 48 & Strong $\mathrm{C}_{2} \mathrm{H}_{5}{ }^{13} \mathrm{CN}$ and $\mathrm{CH}_{3}{ }^{13} \mathrm{CH}_{2} \mathrm{CN}$ \\
\hline 434 & $39_{20,19}-38_{20,18^{\star}}$ & 213984.089 & 9 & 483 & 98.6 & 48 & Strong $\mathrm{C}_{2} \mathrm{H}_{5}{ }^{13} \mathrm{CN}$ and $\mathrm{CH}_{3}{ }^{13} \mathrm{CH}_{2} \mathrm{CN}$ \\
\hline 436 & $39_{19,20}-38_{19,19^{\star}}$ & 213989.994 & 7 & 455 & 102.0 & 48 & Strong $\mathrm{C}_{2} \mathrm{H}_{5}{ }^{13} \mathrm{CN}$ and $\mathrm{CH}_{3}{ }^{13} \mathrm{CH}_{2} \mathrm{CN}$ \\
\hline 438 & $39_{18,21}-38_{18,20}{ }^{\star}$ & 213998.464 & 6 & 428 & 105.3 & 48 & Strong $\mathrm{C}_{2} \mathrm{H}_{5}{ }^{13} \mathrm{CN}$ and $\mathrm{CH}_{3}{ }^{13} \mathrm{CH}_{2} \mathrm{CN}$ \\
\hline 440 & $39_{17,22}-38_{17,21}{ }^{\star}$ & 214010.151 & 5 & 403 & 108.3 & 48 & Strong $\mathrm{C}_{2} \mathrm{H}_{5}{ }^{13} \mathrm{CN}$ and $\mathrm{CH}_{3}{ }^{13} \mathrm{CH}_{2} \mathrm{CN}$ \\
\hline 442 & $39_{16,24}-38_{16,23}{ }^{\star}$ & 214025.894 & 5 & 379 & 111.3 & 48 & Strong $\mathrm{C}_{2} \mathrm{H}_{5}{ }^{13} \mathrm{CN}$ and $\mathrm{CH}_{3}{ }^{13} \mathrm{CH}_{2} \mathrm{CN}$ \\
\hline 444 & $39_{15,24}-38_{15,23}{ }^{\star}$ & 214046.781 & 100 & 357 & 114.0 & 48 & Blend with ${ }^{13} \mathrm{CH}_{3} \mathrm{CN}$ \\
\hline 446 & $39_{14,25}-38_{14,24}{ }^{\star}$ & 214074.316 & 100 & 336 & 116.5 & 75 & Strong $\mathrm{CH}_{3} \mathrm{OCH}_{3}$ and $\mathrm{CH}_{3}{ }^{13} \mathrm{CH}_{2} \mathrm{CN}$ \\
\hline 448 & $39_{13,26}-38_{13,25}{ }^{\star}$ & 214110.717 & 4 & 317 & 118.9 & 75 & Blend with ${ }^{13} \mathrm{CH}_{3} \mathrm{CN}$ \\
\hline 450 & $39_{12,27}-38_{12,26}{ }^{\star}$ & 214158.803 & 100 & 299 & 121.1 & 75 & Blend with $\mathrm{C}_{2} \mathrm{H}_{5}{ }^{13} \mathrm{CN}$ \\
\hline 452 & $39_{11,28}-38_{11,27}{ }^{\star}$ & 214223.159 & 100 & 282 & 123.1 & 75 & Strong $\mathrm{CH}_{2} \mathrm{NH}$ and ${ }^{13} \mathrm{CH}_{3} \mathrm{CN}$ \\
\hline 454 & $39_{10,30}-38_{10,29^{\star}}$ & 214310.764 & 4 & 267 & 125.0 & 75 & Strong ${ }^{13} \mathrm{CH}_{3} \mathrm{CN}$ \\
\hline 456 & $66_{4,62}-66_{3,63}$ & 214317.063 & 27 & 591 & 13.5 & 75 & Strong ${ }^{13} \mathrm{CH}_{3} \mathrm{CN}$ \\
\hline 457 & $9_{6,4}-8_{5,3}{ }^{\star}$ & 214379.791 & 13 & 27 & 2.8 & 75 & Strong ${ }^{13} \mathrm{CH}_{3} \mathrm{CN}$ \\
\hline 459 & $39_{9,31}-38_{9,30}$ * & 214432.762 & 4 & 254 & 126.6 & 75 & Blend with $\mathrm{CH}_{3}{ }^{13} \mathrm{CH}_{2} \mathrm{CN}$ and $\mathrm{C}_{2} \mathrm{H}_{5} \mathrm{OH}$ \\
\hline 461 & $22_{3,19}-21_{2,20}$ & 214433.988 & 14 & 63 & 1.7 & 75 & Blend with $\mathrm{CH}_{3}{ }^{13} \mathrm{CH}_{2} \mathrm{CN}$ and $\mathrm{C}_{2} \mathrm{H}_{5} \mathrm{OH}$ \\
\hline 462 & $65_{5,61}-65_{4,62}$ & 214600.532 & 23 & 574 & 13.4 & 75 & Blend with $\mathrm{CH}_{3} \mathrm{CH}_{3} \mathrm{CO}$ \\
\hline 463 & $394,36-38_{4,35}$ & 214605.781 & 4 & 207 & 132.3 & 75 & Blend with $\mathrm{CH}_{3} \mathrm{CH}_{3} \mathrm{CO}$ and $\mathrm{CH}_{3}{ }^{13} \mathrm{CH}_{2} \mathrm{CN}$ \\
\hline 464 & $56_{8,49}-56_{7,50}$ & 214606.526 & 11 & 457 & 15.7 & 75 & Blend with $\mathrm{CH}_{3} \mathrm{CH}_{3} \mathrm{CO}$ and $\mathrm{CH}_{3}{ }^{13} \mathrm{CH}_{2} \mathrm{CN}$ \\
\hline
\end{tabular}


A. Belloche et al.: Detection and chemical modeling of ethyl formate and $n$-propyl cyanide in Sgr B2(N), Online Material $p 6$

Table 1. continued.

\begin{tabular}{|c|c|c|c|c|c|c|c|}
\hline$N^{a}$ & $\begin{array}{c}\text { Transition }^{b} \\
(2) \\
\end{array}$ & $\begin{array}{c}\begin{array}{c}\text { Frequency } \\
(\mathrm{MHz}) \\
(3)\end{array} \\
\end{array}$ & $\begin{array}{c}\text { Unc. }^{c} \\
(\mathrm{kHz}) \\
(4) \\
\end{array}$ & $\begin{array}{l}E_{1}^{d} \\
(\mathrm{~K}) \\
(5) \\
\end{array}$ & $\begin{array}{l}S \mu^{2} \\
\left(\mathrm{D}^{2}\right) \\
(6) \\
\end{array}$ & $\begin{array}{c}\sigma^{e} \\
(\mathrm{mK}) \\
(7) \\
\end{array}$ & $\begin{array}{c}\text { Comments } \\
(8) \\
\end{array}$ \\
\hline 465 & $39_{8,32}-38_{8,31}$ & 214608.530 & 4 & 241 & 128.1 & 75 & Blend with $\mathrm{CH}_{3}{ }^{13} \mathrm{CH}_{2} \mathrm{CN}$ \\
\hline 466 & $39_{8,31}-38_{8,30}$ & 214609.070 & 4 & 241 & 128.1 & 75 & Blend with $\mathrm{CH}_{3}{ }^{13} \mathrm{CH}_{2} \mathrm{CN}$ \\
\hline 467 & $39_{7,33}-38_{7,32}$ & 214870.593 & 100 & 231 & 129.5 & 75 & Blend with ${ }^{13} \mathrm{CH}_{3} \mathrm{CN}, v_{8}=1$ \\
\hline 468 & $397,32-387,31$ & 214881.820 & 4 & 231 & 129.5 & 75 & Blend with ${ }^{13} \mathrm{CH}_{2} \mathrm{CHCN}$ \\
\hline 469 & $39_{6,34}-38_{6,33}$ & 215238.445 & 100 & 222 & 130.6 & 74 & Strong $\mathrm{C}_{2} \mathrm{H}_{5} \mathrm{CN}, v_{13}=1 / v_{21}=1$ and $\mathrm{C}_{2} \mathrm{H}_{5} \mathrm{CN}, v_{20}=1$ \\
\hline 470 & $20_{4,16}-19_{3,17}$ & 215256.547 & 11 & 57 & 2.9 & 74 & Strong $\mathrm{C}_{2} \mathrm{H}_{5} \mathrm{CN}, v_{13}=1 / v_{21}=1$ and $\mathrm{C}_{2} \mathrm{H}_{5} \mathrm{CN}, v_{20}=1$ \\
\hline 471 & $39_{6,33}-38_{6,32}$ & 215399.020 & 100 & 222 & 130.6 & 74 & Strong $\mathrm{C}_{2} \mathrm{H}_{5} \mathrm{CN}$ \\
\hline 472 & $39_{5,35}-38_{5,34}$ & 215467.027 & 4 & 214 & 131.6 & 74 & Strong $\mathrm{C}_{2} \mathrm{H}_{5} \mathrm{CN}, v_{13}=1 / v_{21}=1$ \\
\hline 473 & $21_{4,18}-20_{3,17}$ & 215732.383 & 12 & 62 & 3.0 & 74 & Strong $\mathrm{C}_{2} \mathrm{H}_{5} \mathrm{CN}, v_{13}=1 / v_{21}=1$ \\
\hline 474 & $39_{5,34}-38_{5,33}$ & 216832.944 & 100 & 214 & 131.6 & 50 & Strong $\mathrm{CH}_{3} \mathrm{OCHO}$ \\
\hline 475 & $61_{3,58}-61_{3,59}$ & 216833.377 & 28 & 498 & 1.2 & 50 & Strong $\mathrm{CH}_{3} \mathrm{OCHO}$ \\
\hline 476 & $41_{2,39}-40_{3,38}$ & 216942.926 & 5 & 222 & 10.6 & 50 & Strong $\mathrm{CH}_{3} \mathrm{OH}$ \\
\hline 477 & $38_{3,36}-37_{2,35}$ & 217039.712 & 8 & 191 & 9.0 & 50 & Blend with ${ }^{13} \mathrm{CH}_{3} \mathrm{OH}$ and ${ }^{13} \mathrm{CN}$ \\
\hline 478 & $40_{3,38}-39_{3,37}$ & 217052.822 & 100 & 211 & 136.1 & 50 & Blend with ${ }^{13} \mathrm{CH}_{3} \mathrm{OH},{ }^{13} \mathrm{CN}$, and $U$-line \\
\hline 479 & $15_{5,11}-14_{4,10}$ & 217159.548 & 12 & 39 & 3.0 & 50 & Blend with $\mathrm{CH}_{3} \mathrm{OCH}_{3}$ \\
\hline 480 & $15_{5,10}-14_{4,11}$ & 217179.901 & 12 & 39 & 3.0 & 50 & Blend with $\mathrm{CH}_{3} \mathrm{OCH}_{3}$ and $U$-line \\
\hline 481 & $41_{1,40}-40_{2,39}$ & 217679.824 & 4 & 215 & 14.7 & 50 & Blend with $U$-line \\
\hline 482 & $39_{4,35}-38_{4,34}$ & 219402.429 & 4 & 209 & 132.5 & 92 & Strong $\mathrm{C}_{2} \mathrm{H}_{3} \mathrm{CN}$ \\
\hline 483 & $40_{24,16}-39_{24,15}^{\star}$ & 219460.441 & 43 & 619 & 87.8 & 92 & Strong $\mathrm{C}_{2} \mathrm{H}_{5} \mathrm{CN}$ \\
\hline 485 & $40_{25,15}-39_{25,14^{\star}}$ & 219460.780 & 60 & 655 & 83.6 & 92 & Strong $\mathrm{C}_{2} \mathrm{H}_{5} \mathrm{CN}$ \\
\hline 487 & $40_{23,17}-39_{23,16^{\star}}$ & 219460.864 & 30 & 586 & 91.8 & 92 & Strong $\mathrm{C}_{2} \mathrm{H}_{5} \mathrm{CN}$ \\
\hline 489 & $40_{26,14}-39_{26,13^{\star}}$ & 219461.621 & 83 & 691 & 79.2 & 92 & Strong $\mathrm{C}_{2} \mathrm{H}_{5} \mathrm{CN}$ \\
\hline 491 & $40_{22,18}-39_{22,17^{\star}}$ & 219462.336 & 20 & 553 & 95.7 & 92 & Strong $\mathrm{C}_{2} \mathrm{H}_{5} \mathrm{CN}$ \\
\hline 493 & $40_{27,13}-39_{27,12^{\star}}$ & 219462.721 & 112 & 729 & 74.7 & 92 & Strong $\mathrm{C}_{2} \mathrm{H}_{5} \mathrm{CN}$ \\
\hline 495 & $40_{28,12}-39_{28,11^{\star}}$ & 219463.851 & 149 & 768 & 70.0 & 92 & Strong $\mathrm{C}_{2} \mathrm{H}_{5} \mathrm{CN}$ \\
\hline 497 & $40_{21,19}-39_{21,18}$ & 219465.183 & 14 & 522 & 99.4 & 92 & Strong $\mathrm{C}_{2} \mathrm{H}_{5} \mathrm{CN}$ \\
\hline 498 & $40_{21,20}-39_{21,19}$ & 219465.523 & 100 & 522 & 99.4 & 92 & Strong $\mathrm{C}_{2} \mathrm{H}_{5} \mathrm{CN}$ \\
\hline 499 & $40_{20,20}-39_{20,19}$ & 219469.782 & 10 & 493 & 102.9 & 92 & Strong $\mathrm{NH}_{2} \mathrm{CN}$ \\
\hline 500 & $40_{20,21}-39_{20,20}$ & 219469.912 & 100 & 493 & 102.9 & 92 & Strong $\mathrm{NH}_{2} \mathrm{CN}$ \\
\hline 501 & $40_{19,21}-39_{19,20}{ }^{\star}$ & 219476.588 & 7 & 465 & 106.3 & 92 & Strong $\mathrm{NH}_{2} \mathrm{CN}$ \\
\hline 503 & $40_{18,22}-39_{18,21}$ & 219486.155 & 6 & 438 & 109.4 & 92 & Strong $\mathrm{HC}_{3} \mathrm{~N}, v_{6}=v_{7}=1$ \\
\hline 504 & $40_{18,23}-39_{18,22}$ & 219486.310 & 100 & 438 & 109.4 & 92 & Strong $\mathrm{HC}_{3} \mathrm{~N}, v_{6}=v_{7}=1$ \\
\hline 505 & $40_{17,23}-39_{17,22}$ & 219499.182 & 5 & 413 & 112.4 & 92 & Strong $\mathrm{C}_{2} \mathrm{H}_{5} \mathrm{CN}$ \\
\hline 506 & $40_{17,24}-39_{17,23}$ & 219499.329 & 100 & 413 & 112.4 & 92 & Strong $\mathrm{C}_{2} \mathrm{H}_{5} \mathrm{CN}$ \\
\hline 507 & $40_{16,25}-39_{16,24}{ }^{\star}$ & 219516.573 & 5 & 390 & 115.3 & 92 & Strong $\mathrm{HC}_{3} \mathrm{~N}, v_{6}=v_{7}=1$ \\
\hline 509 & $40_{15,25}-39_{15,24}{ }^{\star}$ & 219539.521 & 5 & 367 & 117.9 & 92 & Strong $\mathrm{HNCO}$ and $\mathrm{HNCO}, v_{5}=1$ \\
\hline 511 & $40_{14,26}-39_{14,25}{ }^{\star}$ & 219569.648 & 5 & 346 & 120.4 & 92 & Strong $\mathrm{C}^{18} \mathrm{O}$ \\
\hline 513 & $67_{4,63}-67_{3,64}$ & 219571.157 & 30 & 609 & 13.4 & 92 & Strong $\mathrm{C}^{18} \mathrm{O}$ \\
\hline 514 & $40_{13,27}-39_{13,26}{ }^{\star}$ & 219609.218 & 5 & 327 & 122.7 & 92 & Strong $\mathrm{C}_{2} \mathrm{H}_{3} \mathrm{CN}, v_{15}=1$ and $\mathrm{CH}_{2} \mathrm{CH}^{13} \mathrm{CN}$ \\
\hline 516 & $40_{12,28}-39_{12,27}$ & 219661.381 & 100 & 309 & 124.8 & 92 & Strong $\mathrm{HNCO}$ and $\mathrm{HC}_{3} \mathrm{~N}, v_{7}=2$ \\
\hline 517 & $40_{12,29}-39_{12,28}$ & 219661.485 & 5 & 309 & 124.8 & 92 & Strong $\mathrm{HNCO}$ and $\mathrm{HC}_{3} \mathrm{~N}, v_{7}=2$ \\
\hline 518 & $40_{11,30}-39_{11,29}{ }^{\star}$ & 219731.290 & 4 & 293 & 126.8 & 92 & Strong HNCO \\
\hline 520 & $40_{7,34}-39_{7,33}$ & 220431.537 & 100 & 241 & 133.0 & 98 & Strong $\mathrm{CH}_{3}{ }^{13} \mathrm{CN}$ \\
\hline 521 & $40_{7,33}-39_{7,32}$ & 220446.964 & 100 & 241 & 133.0 & 98 & Blend with $U$-line, uncertain baseline \\
\hline 522 & $41_{7,35}-40_{7,34}$ & 225996.244 & 100 & 252 & 136.5 & 278 & Blend with $\mathrm{CH}_{3} \mathrm{CH}_{3} \mathrm{CO}, v_{t}=1$, uncertain baseline \\
\hline 523 & $41_{7,34}-40_{7,33}$ & 226017.388 & 100 & 252 & 136.5 & 278 & Blend with $U$-line?, uncertain baseline \\
\hline 524 & $63_{3,60}-63_{2,61}$ & 226393.597 & 33 & 530 & 10.0 & 278 & Blend with $\mathrm{CN}$ in absorption, $\mathrm{C}_{2} \mathrm{H}_{3} \mathrm{CN}$, and $\mathrm{C}_{2} \mathrm{H}_{5} \mathrm{OH}$ \\
\hline 525 & $41_{6,36}-40_{6,35}$ & 226400.096 & 100 & 243 & 137.6 & 278 & Blend with $\mathrm{CN}$ in absorption and $\mathrm{C}_{2} \mathrm{H}_{5} \mathrm{OH}$ \\
\hline 526 & $41_{5,37}-40_{5,36}$ & 226540.631 & 4 & 235 & 138.5 & 278 & Blend with $\mathrm{CH}_{2} \mathrm{NH}$ \\
\hline 527 & $41_{6,35}-40_{6,34}$ & 226668.561 & 4 & 243 & 137.6 & 96 & Strong $\mathrm{CN}$ absorption \\
\hline 528 & $22_{4,18}-21_{3,19}$ & 226986.056 & 11 & 67 & 3.0 & 96 & Strong CN absorption \\
\hline 529 & $42_{3,40}-41_{3,39}$ & 227467.207 & 100 & 233 & 143.0 & 85 & Blend with $\mathrm{CH}_{2}(\mathrm{OH}) \mathrm{CHO}, \mathrm{CH}_{2} \mathrm{CH}^{13} \mathrm{CN}$, and $\mathrm{C}_{3} \mathrm{H}_{7} \mathrm{CN}$ \\
\hline 530 & $24_{4,21}-23_{3,20}$ & 227801.331 & 12 & 80 & 3.2 & 85 & Strong $\mathrm{CH}_{3} \mathrm{OH}$ \\
\hline 531 & $42_{9,34}-41_{9,33}{ }^{\star}$ & 231015.633 & 5 & 285 & 137.4 & 183 & Blend with $\mathrm{CH}_{3} \mathrm{OCHO}, \mathrm{H}_{2}{ }^{13} \mathrm{CS}$, and $U$-line \\
\hline 533 & $42_{8,35}-41_{8,34}$ & 231237.570 & 5 & 273 & 138.8 & 183 & Strong ${ }^{13} \mathrm{CH}_{2} \mathrm{CHCN}$ and $\mathrm{C}_{2} \mathrm{H}_{3} \mathrm{CN}, v_{15}=1$ \\
\hline 534 & $42_{8,34}-41_{8,33}$ & 231239.195 & 5 & 273 & 138.8 & 183 & Strong ${ }^{13} \mathrm{CH}_{2} \mathrm{CHCN}$ and $\mathrm{C}_{2} \mathrm{H}_{3} \mathrm{CN}, v_{15}=1$ \\
\hline 535 & $25_{4,22}-24_{3,21}$ & 231328.525 & 13 & 86 & 3.3 & 40 & Blend with $\mathrm{CH}_{3} \mathrm{CHO}$ and ${ }^{13} \mathrm{CH}_{2} \mathrm{CHCN}$ \\
\hline 536 & $42_{7,36}-41_{7,35}$ & 231564.827 & 4 & 262 & 140.1 & 40 & Blend with $\mathrm{C}_{2} \mathrm{H}_{5} \mathrm{OH}$ \\
\hline 537 & $42_{7,35}-41_{7,34}$ & 231593.484 & 100 & 263 & 140.1 & 40 & Blend with $\mathrm{CH}_{3}{ }^{13} \mathrm{CH}_{2} \mathrm{CN}$ \\
\hline 538 & $43_{3,41}-42_{3,40}$ & 232661.017 & 5 & 243 & 146.4 & 19 & Blend with $\mathrm{C}_{2} \mathrm{H}_{5} \mathrm{CN}, v_{13}=1 / v_{21}=1$ \\
\hline 539 & $42_{3,40}-41_{2,39}$ & 232789.078 & 6 & 232 & 11.2 & 19 & Strong $\mathrm{CH}_{3} \mathrm{OH}, \mathrm{C}_{2} \mathrm{H}_{5} \mathrm{CN}$, and $\mathrm{C}_{2} \mathrm{H}_{3} \mathrm{CN}, v_{11}=1$ \\
\hline 540 & $43_{26,17}-42_{26,16^{\star}}$ & 235907.995 & 89 & 723 & 93.6 & 131 & Blend with $\mathrm{HCC}^{13} \mathrm{CN}, v_{6}=1$ \\
\hline 542 & $43_{27,16}-42_{27,15}^{\star}$ & 235908.172 & 120 & 761 & 89.3 & 131 & Blend with $\mathrm{HCC}^{13} \mathrm{CN}, v_{6}=1$ \\
\hline
\end{tabular}


Table 1. continued.

\begin{tabular}{|c|c|c|c|c|c|c|c|}
\hline$N^{a}$ & $\begin{array}{c}\text { Transition }^{b} \\
\text { (2) }\end{array}$ & $\begin{array}{c}\text { Frequency } \\
(\mathrm{MHz}) \\
(3)\end{array}$ & $\begin{array}{c}\text { Unc. }^{c} \\
(\mathrm{kHz}) \\
(4) \\
\end{array}$ & $\begin{array}{l}E_{1}^{d} \\
(\mathrm{~K}) \\
(5) \\
\end{array}$ & $\begin{array}{l}S \mu^{2} \\
\left(\mathrm{D}^{2}\right) \\
(6) \\
\end{array}$ & $\begin{array}{c}\sigma^{e} \\
(\mathrm{mK}) \\
(7) \\
\end{array}$ & $\begin{array}{c}\text { Comments } \\
(8) \\
\end{array}$ \\
\hline 544 & $43_{25,18}-42_{25,17}{ }^{\star}$ & 235908.220 & 65 & 687 & 97.6 & 131 & Blend with $\mathrm{HCC}^{13} \mathrm{CN}, v_{6}=1$ \\
\hline 546 & $43_{24,19}-42_{24,18}{ }^{\star}$ & 235909.130 & 46 & 652 & 101.6 & 131 & Blend with $\mathrm{HCC}^{13} \mathrm{CN}, v_{6}=1$ \\
\hline 548 & $43_{23,20}-42_{23,19}{ }^{\star}$ & 235911.029 & 32 & 618 & 105.3 & 131 & Blend with $\mathrm{HCC}^{13} \mathrm{CN}, v_{6}=1$ \\
\hline 550 & $43_{22,22}-42_{22,21}$ * & 235914.238 & 100 & 586 & 108.9 & 131 & Strong $\mathrm{CH}_{3} \mathrm{OCHO}$ \\
\hline 552 & $43_{21,22}-42_{21,21}^{\star}$ & 235919.208 & 15 & 555 & 112.3 & 131 & Strong $\mathrm{CH}_{3} \mathrm{OCHO}$ \\
\hline 554 & $43_{20,23}-42_{20,22}$ & 235926.335 & 10 & 525 & 115.6 & 131 & Strong $\mathrm{CH}_{3} \mathrm{OCHO}, \mathrm{C}_{2} \mathrm{H}_{3} \mathrm{CN}, v_{15}=1$, and ${ }^{13} \mathrm{CH}_{3} \mathrm{OH}$ \\
\hline 555 & $43_{20,24}-42_{20,23}$ & 235926.477 & 100 & 525 & 115.6 & 131 & Strong $\mathrm{CH}_{3} \mathrm{OCHO}, \mathrm{C}_{2} \mathrm{H}_{3} \mathrm{CN}, v_{15}=1$, and ${ }^{13} \mathrm{CH}_{3} \mathrm{OH}$ \\
\hline 556 & $43_{19,24}-42_{19,23}{ }^{\star}$ & 235936.190 & 8 & 497 & 118.7 & 131 & Strong $\mathrm{CH}_{3} \mathrm{OCHO}, \mathrm{C}_{2} \mathrm{H}_{3} \mathrm{CN}, v_{15}=1$, and ${ }^{13} \mathrm{CH}_{3} \mathrm{OH}$ \\
\hline 558 & $43_{18,25}-42_{18,24}^{\star}$ & 235949.452 & 6 & 471 & 121.6 & 131 & Strong $\mathrm{C}_{2} \mathrm{H}_{3} \mathrm{CN}, v_{15}=1,{ }^{13} \mathrm{CH}_{3} \mathrm{OH}$, and $\mathrm{C}_{2} \mathrm{H}_{5}{ }^{13} \mathrm{CN}$ \\
\hline 560 & $43_{17,26}-42_{17,25}^{\star}$ & 235966.980 & 6 & 446 & 124.4 & 131 & Strong ${ }^{13} \mathrm{CH}_{3} \mathrm{OH}$ and $\mathrm{SiS}$ \\
\hline 562 & $43_{16,27}-42_{16,26}{ }^{\star}$ & 235989.879 & 100 & 422 & 127.1 & 131 & Strong ${ }^{13} \mathrm{CH}_{3} \mathrm{OH}$ \\
\hline 564 & $43_{13,31}-42_{13,30}{ }^{\star}$ & 236108.561 & 100 & 359 & 134.0 & 37 & Blend with $\mathrm{C}_{2} \mathrm{H}_{5} \mathrm{CN}, v_{13}=1 / v_{21}=1$ and $\mathrm{NH}_{2} \mathrm{CH}_{2} \mathrm{CN}$ \\
\hline 566 & $43_{12,31}-42_{12,30}{ }^{\star}$ & 236174.724 & 5 & 342 & 136.0 & 37 & Blend with ${ }^{13} \mathrm{CH}_{3} \mathrm{CH}_{2} \mathrm{CN}$ and $\mathrm{CH}_{2}{ }^{13} \mathrm{CHCN}$ \\
\hline 568 & $43_{11,32}-42_{11,31}$ * & 236262.562 & 100 & 325 & 137.9 & 37 & Blend with $\mathrm{C}_{2} \mathrm{H}_{5} \mathrm{CN}, v_{13}=1 / v_{21}=1$ and $\mathrm{NH}_{2} \mathrm{CH}_{2} \mathrm{CN}$ \\
\hline 570 & $13_{6,8}-12_{5,7}{ }^{\star}$ & 236300.957 & 13 & 39 & 3.1 & 37 & Strong $\mathrm{CH}_{2}{ }^{13} \mathrm{CHCN}$ and $\mathrm{C}_{2} \mathrm{H}_{5} \mathrm{OH}$ \\
\hline 572 & $65_{4,62}-65_{3,63}$ & 236306.402 & 38 & 563 & 10.0 & 37 & Strong ${ }^{13} \mathrm{CH}_{3} \mathrm{CH}_{2} \mathrm{CN}$ \\
\hline 573 & $43_{10,33}-42_{10,32^{\star}}$ & 236381.716 & 100 & 310 & 139.5 & 37 & Blend with $\mathrm{CH}_{2}{ }^{13} \mathrm{CHCN}$ \\
\hline 575 & $43_{9,35}-42_{9,34}$ & 236547.689 & 5 & 296 & 141.0 & 37 & Blend with ${ }^{13} \mathrm{CH}_{3} \mathrm{CH}_{2} \mathrm{CN}$ \\
\hline 576 & $43_{9,34}-42_{9,33}$ & 236547.789 & 5 & 296 & 141.0 & 37 & Blend with ${ }^{13} \mathrm{CH}_{3} \mathrm{CH}_{2} \mathrm{CN}$ \\
\hline 577 & $20_{5,16}-19_{4,15}$ & 244080.041 & 12 & 62 & 3.4 & 46 & Blend with $\mathrm{CH}_{3} \mathrm{CH}_{3} \mathrm{CO}, v_{t}=1$ \\
\hline 578 & $20_{5,15}-19_{4,16}$ & 244307.468 & 12 & 62 & 3.4 & 46 & Blend with $\mathrm{CH}_{3} \mathrm{OH}$ and $\mathrm{C}_{2} \mathrm{H}_{5}{ }^{13} \mathrm{CN}$ \\
\hline 579 & $9_{7,2}-8_{6,3}{ }^{\star}$ & 244368.037 & 13 & 35 & 3.2 & 46 & Blend with $\mathrm{C}_{2} \mathrm{H}_{5} \mathrm{CN}$ \\
\hline 581 & $47_{0,47}-46_{1,46}$ & 244480.036 & 7 & 272 & 21.2 & 91 & Blend with ${ }^{34} \mathrm{SO}_{2}$ \\
\hline 582 & $47_{1,47}-46_{1,46}$ & 244482.013 & 7 & 272 & 161.0 & 91 & Blend with ${ }^{34} \mathrm{SO}_{2}$ \\
\hline 583 & $47_{0,47}-46_{0,46}$ & 244482.600 & 7 & 272 & 161.0 & 91 & Blend with ${ }^{34} \mathrm{SO}_{2}$ \\
\hline 584 & $47_{1,47}-46_{0,46}$ & 244484.577 & 7 & 272 & 21.2 & 91 & Blend with ${ }^{34} \mathrm{SO}_{2}$ \\
\hline 585 & $44_{3,41}-43_{3,40}$ & 244500.437 & 5 & 260 & 149.8 & 91 & Blend with $\mathrm{CH}_{3} \mathrm{OCH}_{3}$ \\
\hline 586 & $30_{4,27}-293,26$ & 245163.234 & 16 & 123 & 3.8 & 72 & Strong $\mathrm{HCC}^{13} \mathrm{CN}, v_{7}=1$ \\
\hline 587 & $25_{4,21}-24_{3,22}$ & 245723.730 & 11 & 86 & 3.0 & 53 & Blend with $\mathrm{CH}_{2}{ }^{13} \mathrm{CHCN}$ \\
\hline 588 & $45_{17,28}-44_{17,27^{\star}}$ & 246946.103 & 100 & 469 & 132.3 & 68 & Strong $\mathrm{C}_{2} \mathrm{H}_{3} \mathrm{CN}$ \\
\hline 590 & $45_{16,29}-44_{16,28}{ }^{\star}$ & 246973.216 & 6 & 445 & 134.8 & 68 & Blend with $\mathrm{C}_{2} \mathrm{H}_{5} \mathrm{CN}$ and $\mathrm{CH}_{3} \mathrm{COOH}$ \\
\hline 592 & $45_{15,30}-44_{15,29}{ }^{\star}$ & 247008.106 & 100 & 423 & 137.2 & 68 & Strong $\mathrm{C}_{2} \mathrm{H}_{3} \mathrm{CN}$ \\
\hline 594 & $45_{14,31}-44_{14,30}$ * & 247053.206 & 100 & 402 & 139.4 & 68 & Strong $\mathrm{CH}_{3} \mathrm{OCHO}$ \\
\hline 596 & $45_{13,33}-44_{13,32}{ }^{\star}$ & 247111.643 & 100 & 382 & 141.5 & 68 & Strong $\mathrm{HC}_{3} \mathrm{~N}, v_{7}=2$ and $\mathrm{CH}_{3} \mathrm{OCHO}$ \\
\hline 598 & $45_{12,34}-44_{12,33^{\star}}$ & 247188.187 & 5 & 364 & 143.4 & 68 & Blend with $\mathrm{C}_{2} \mathrm{H}_{3} \mathrm{CN}, \mathrm{CH}_{3} \mathrm{CH}_{3} \mathrm{CO}, v_{t}=1$ and $\mathrm{CH}_{3} \mathrm{COOH}$ \\
\hline 600 & $15_{6,10}-14_{5,9}$ & 247242.428 & 12 & 46 & 3.3 & 68 & Strong $\mathrm{C}_{2} \mathrm{H}_{3} \mathrm{CN}, v_{15}=1$ and $\mathrm{CH}_{3}{ }^{13} \mathrm{CH}_{2} \mathrm{CN}$ \\
\hline 601 & $15_{6,9}-14_{5,10}$ & 247242.754 & 12 & 46 & 3.3 & 68 & Strong $\mathrm{C}_{2} \mathrm{H}_{3} \mathrm{CN}, v_{15}=1$ and $\mathrm{CH}_{3}{ }^{13} \mathrm{CH}_{2} \mathrm{CN}$ \\
\hline 602 & $31_{4,28}-30_{3,27}$ & 247248.744 & 16 & 131 & 3.9 & 68 & Strong $\mathrm{C}_{2} \mathrm{H}_{3} \mathrm{CN}, v_{15}=1$ and $\mathrm{CH}_{3}{ }^{13} \mathrm{CH}_{2} \mathrm{CN}$ \\
\hline 603 & $45_{11,35}-44_{11,34}{ }^{\star}$ & 247289.755 & 5 & 348 & 145.1 & 68 & Blend with $\mathrm{C}_{2} \mathrm{H}_{3} \mathrm{CN}, v_{15}=1$ \\
\hline 605 & $56_{9,48}-56_{8,49}$ & 247291.902 & 12 & 468 & 15.2 & 68 & Blend with $\mathrm{C}_{2} \mathrm{H}_{3} \mathrm{CN}, v_{15}=1$ \\
\hline 606 & $45_{10,36}-44_{10,35}{ }^{\star}$ & 247427.317 & 5 & 333 & 146.7 & 68 & Blend with $\mathrm{H}^{13} \mathrm{CCCN}, v_{6}=1$ and $\mathrm{t}-\mathrm{HCOOH}$ \\
\hline 608 & $46_{4,43}-45_{4,42}$ & 251970.749 & 5 & 285 & 156.4 & 42 & Blend with $\mathrm{CH}_{3} \mathrm{OCH}_{3}$ and $\mathrm{CH}_{3} \mathrm{OH}$ \\
\hline 609 & $45_{5,40}-44_{5,39}$ & 252031.184 & 100 & 281 & 152.5 & 42 & Blend with $\mathrm{C}_{2} \mathrm{H}_{5} \mathrm{CN}, v_{13}=1 / v_{21}=1$ \\
\hline 610 & $46_{21,25}-45_{21,24}$ & 252371.919 & 16 & 590 & 124.9 & 42 & Strong ${ }^{13} \mathrm{CH}_{3} \mathrm{OH}$ \\
\hline 611 & $46_{21,26}-45_{21,25}$ & 252372.057 & 100 & 590 & 124.9 & 42 & Strong ${ }^{13} \mathrm{CH}_{3} \mathrm{OH}$ \\
\hline 612 & $46_{20,26}-45_{20,25}$ & 252382.043 & 11 & 560 & 128.0 & 42 & Blend with ${ }^{13} \mathrm{CH}_{3} \mathrm{CH}_{2} \mathrm{CN}$ \\
\hline 613 & $46_{20,27}-45_{20,26}$ & 252382.165 & 100 & 560 & 128.0 & 42 & Blend with ${ }^{13} \mathrm{CH}_{3} \mathrm{CH}_{2} \mathrm{CN}$ \\
\hline 614 & $46_{19,27}-45_{19,26}$ & 252395.494 & 8 & 532 & 130.9 & 42 & Strong $\mathrm{NH}_{2} \mathrm{CHO}$ and $\mathrm{C}_{2} \mathrm{H}_{3} \mathrm{CN}, v_{11}=1$ \\
\hline 615 & $46_{19,28}-45_{19,27}$ & 252395.596 & 100 & 532 & 130.9 & 42 & Strong $\mathrm{NH}_{2} \mathrm{CHO}$ and $\mathrm{C}_{2} \mathrm{H}_{3} \mathrm{CN}, v_{11}=1$ \\
\hline 616 & $26_{4,22}-25_{3,23}$ & 252401.668 & 100 & 92 & 3.0 & 42 & Strong $\mathrm{NH}_{2} \mathrm{CHO}$ and $\mathrm{C}_{2} \mathrm{H}_{3} \mathrm{CN}, v_{11}=1$ \\
\hline 617 & $46_{18,28}-45_{18,27}^{\star}$ & 252413.093 & 7 & 506 & 133.6 & 42 & Blend with $\mathrm{C}_{2} \mathrm{H}_{3} \mathrm{CN}, v_{11}=1$ and $U$-line \\
\hline 619 & $46_{17,30}-45_{17,29}{ }^{\star}$ & 252435.886 & 6 & 480 & 136.2 & 42 & Strong $\mathrm{C}_{2} \mathrm{H}_{5} \mathrm{CN}, v_{13}=1 / v_{21}=1$ \\
\hline 621 & $46_{16,31}-45_{16,30}$ & 252465.229 & 6 & 457 & 138.7 & 42 & Strong $U$-line \\
\hline 622 & $46_{16,30}-45_{16,29}$ & 252465.379 & 100 & 457 & 138.7 & 42 & Strong $U$-line \\
\hline 623 & $93_{7,86}-93_{6,87}$ & 252466.454 & 90 & 1191 & 24.1 & 42 & Strong $U$-line \\
\hline 624 & $34_{9,25}-34_{8,26}$ & 253951.681 & 13 & 203 & 8.3 & 32 & Strong $\mathrm{C}_{2} \mathrm{H}_{5} \mathrm{CN}$ and $\mathrm{SO}_{2}$ \\
\hline 625 & $34_{9,26}-34_{8,27}$ & 253951.859 & 13 & 203 & 8.3 & 32 & Strong $\mathrm{C}_{2} \mathrm{H}_{5} \mathrm{CN}$ and $\mathrm{SO}_{2}$ \\
\hline 626 & $46_{7,39}-45_{7,38}$ & 253965.040 & 5 & 309 & 154.1 & 32 & Strong NS \\
\hline 627 & $48_{1,47}-47_{2,46}$ & 254009.911 & 6 & 293 & 18.1 & 32 & Strong $\mathrm{CH}_{3} \mathrm{OH}$ in absorption \\
\hline 628 & $35_{4,32}-34_{3,31}$ & 254021.313 & 17 & 166 & 4.6 & 32 & Strong $\mathrm{CH}_{3} \mathrm{OH}$ in absorption \\
\hline 629 & $46_{5,42}-45_{5,41}$ & 254033.953 & 5 & 292 & 155.8 & 32 & Blend with $\mathrm{HC}^{13} \mathrm{CCN}, v_{6}=1$ and $\mathrm{C}_{2} \mathrm{H}_{5} \mathrm{OH}$ \\
\hline 630 & $33_{9,24}-33_{8,25}$ & 254066.133 & 13 & 194 & 8.0 & 32 & Blend with $\mathrm{C}_{2} \mathrm{H}_{5} \mathrm{CN}$ \\
\hline 631 & $33_{9,25}-33_{8,26}$ & 254066.244 & 13 & 194 & 8.0 & 32 & Blend with $\mathrm{C}_{2} \mathrm{H}_{5} \mathrm{CN}$ \\
\hline
\end{tabular}


Table 1. continued.

\begin{tabular}{|c|c|c|c|c|c|c|c|}
\hline$N^{a}$ & Transition $^{b}$ & $\begin{array}{c}\text { Frequency } \\
\text { (MHz) } \\
\text { (3) }\end{array}$ & $\begin{array}{l}\text { Unc. }^{c} \\
(\mathrm{kHz}) \\
(4)\end{array}$ & $\begin{array}{l}E_{1}^{d} \\
(\mathrm{~K}) \\
(5)\end{array}$ & $\begin{array}{l}S \mu^{2} \\
\left(\mathrm{D}^{2}\right) \\
(6)\end{array}$ & $\begin{array}{l}\sigma^{e} \\
(\mathrm{mK}) \\
(7)\end{array}$ & $\begin{array}{c}\text { Comments } \\
\text { (8) }\end{array}$ \\
\hline 632 & $48_{2,47}-47_{2,46}$ & 254081.225 & 6 & 293 & 163.9 & 32 & Strong $\mathrm{CH}_{3} \mathrm{CH}_{3} \mathrm{CO}$ and $\mathrm{C}_{2} \mathrm{H}_{5} \mathrm{CN}$ \\
\hline 633 & $86_{6,80}-86_{5,81}^{2,40}$ & 254082.060 & 64 & 1013 & 20.4 & 32 & Strong $\mathrm{CH}_{3} \mathrm{CH}_{3} \mathrm{CO}$ and $\mathrm{C}_{2} \mathrm{H}_{5} \mathrm{CN}$ \\
\hline 634 & $48_{1,47}-47_{1,46}$ & 254100.087 & 6 & 293 & 164.0 & 32 & Blend with SiS \\
\hline 635 & $32_{9,23}-32_{8,24}^{\star}$ & 254170.380 & 13 & 185 & 7.7 & 32 & Blend with ${ }^{13} \mathrm{CH}_{3} \mathrm{CH}_{2} \mathrm{CN}$ \\
\hline 637 & $48_{2,47}-47_{1,46}$ & 254171.401 & 6 & 293 & 18.1 & 32 & Blend with ${ }^{13} \mathrm{CH}_{3} \mathrm{CH}_{2} \mathrm{CN}$ \\
\hline 638 & $31_{9,22}-31_{8,23}$ * & 254265.069 & 13 & 177 & 7.4 & 32 & Strong $\mathrm{HCC}^{13} \mathrm{CN}, v_{7}=1$ and $\mathrm{SO}_{2}$ \\
\hline 640 & $46_{6,41}-45_{6,40}$ & 254311.469 & 5 & 300 & 155.1 & 32 & Strong $\mathrm{C}_{2} \mathrm{H}_{5} \mathrm{CN}$ and ${ }^{13} \mathrm{CH}_{3} \mathrm{CH}_{2} \mathrm{CN}$ \\
\hline 641 & $30_{9,21}-30_{8,22}^{\star}$ & 254350.818 & 13 & 169 & 7.2 & 32 & Strong $\mathrm{NH}_{2}{ }^{13} \mathrm{CHO}$ \\
\hline 643 & $89_{8,82}-89_{7,83}$ & 254352.623 & 46 & 1095 & 23.7 & 32 & Strong $\mathrm{NH}_{2}{ }^{13} \mathrm{CHO}$ \\
\hline 644 & $29_{9,20}-29_{8,21}$ * & 254428.220 & 13 & 161 & 6.9 & 32 & Strong $\mathrm{CH}_{3} \mathrm{OH}$ \\
\hline 646 & $11_{7,5}-10_{6,4}{ }^{\star}$ & 255337.696 & 13 & 40 & 3.4 & 217 & Strong $\mathrm{HC}_{3} \mathrm{~N}, v_{7}=1$ \\
\hline 648 & $47_{3,45}-46_{2,44}$ & 255440.594 & 6 & 289 & 13.8 & 217 & Strong $\mathrm{CH}_{2} \mathrm{CH}^{13} \mathrm{CN}$ \\
\hline 649 & $36_{4,33}-35_{3,32}$ & 255457.676 & 18 & 175 & 4.9 & 217 & Strong $\mathrm{C}_{2} \mathrm{H}_{5} \mathrm{CN}, v_{13}=1 / v_{21}=1$ and $\mathrm{CH}_{2}{ }^{13} \mathrm{CHCN}$ \\
\hline 650 & $47_{18,29}-46_{18,28^{\star}}$ & 257901.052 & 7 & 518 & 137.6 & 1127 & Blend with $\mathrm{CH}_{3} \mathrm{CN}, v_{8}=1$ \\
\hline 652 & $47_{17,30}-46_{17,29}{ }^{\star}$ & 257925.778 & 6 & 493 & 140.1 & 1127 & Noisy, blend with $\mathrm{C}_{2} \mathrm{H}_{3} \mathrm{CN}, v_{11}=1 / v_{15}=1$ and $\mathrm{CH}_{3} \mathrm{OCHO}$ \\
\hline 654 & $46_{5,41}-45_{5,40}$ & 257933.505 & 5 & 293 & 156.0 & 1127 & Noisy, blend with $\mathrm{C}_{2} \mathrm{H}_{3} \mathrm{CN}, v_{11}=1 / v_{15}=1$ and $\mathrm{CH}_{3} \mathrm{OCHO}$ \\
\hline 655 & $47_{16,31}-46_{16,30}$ * & 257957.480 & 6 & 469 & 142.5 & 1127 & Blend with $\mathrm{C}_{2} \mathrm{H}_{5}{ }^{13} \mathrm{CN}$ \\
\hline 657 & $47_{15,32}-46_{15,31}$ * & 257998.085 & 6 & 447 & 144.8 & 1127 & Strong $\mathrm{CH}_{3} \mathrm{CN}, v_{8}=1$ and $\mathrm{CH}_{3} \mathrm{OCHO}$ \\
\hline 659 & $47_{14,33}-46_{14,32}$ * & 258050.228 & 6 & 426 & 146.9 & 1127 & Strong $\mathrm{CH}_{3} \mathrm{CN}, v_{8}=1$ \\
\hline 661 & $47_{13,34}-46_{13,33}{ }^{\star}$ & 258117.607 & 6 & 406 & 148.9 & 1127 & Strong $\mathrm{CH}_{3} \mathrm{OCHO}$ \\
\hline 663 & $17_{6,12}-16_{5,11}$ & 258162.597 & 12 & 54 & 3.5 & 1127 & Strong ${ }^{13} \mathrm{CH}_{3} \mathrm{OH}$ and $\mathrm{HC}^{15} \mathrm{~N}$ \\
\hline 664 & $17_{6,11}-16_{5,12}$ & 258163.845 & 12 & 54 & 3.5 & 1127 & Strong ${ }^{13} \mathrm{CH}_{3} \mathrm{OH}$ and $\mathrm{HC}^{15} \mathrm{~N}$ \\
\hline 665 & $47_{12,35}-46_{12,34}{ }^{\star}$ & 258205.577 & 6 & 388 & 150.7 & 1127 & Strong $\mathrm{CH}_{3} \mathrm{CN}, v_{8}=1$ \\
\hline 667 & $38_{4,35}-37_{3,34}$ & 258232.157 & 17 & 195 & 5.4 & 1127 & Strong $\mathrm{CH}_{3} \mathrm{CN}, v_{8}=1$ \\
\hline 668 & $47_{11,37}-46_{11,36}{ }^{\star}$ & 258322.177 & 6 & 372 & 152.4 & 1127 & Strong $\mathrm{CH}_{3} \mathrm{CN}, v_{8}=1$ \\
\hline 670 & $48_{2,46}-47_{2,45}$ & 258838.876 & 6 & 302 & 163.6 & 1609 & Noisy \\
\hline 671 & $46_{4,42}-45_{4,41}$ & 258951.138 & 5 & 288 & 156.6 & 1609 & Strong $\mathrm{CH}_{3} \mathrm{CN}, v_{8}=2$ \\
\hline 672 & $70_{4,67}-70_{3,68}$ & 259007.596 & 58 & 650 & 10.0 & 1609 & Blend with $\mathrm{H}^{13} \mathrm{CN}$ in absorption and $\mathrm{CH}_{3} \mathrm{CN}, v_{8}=2$ \\
\hline 673 & $47_{8,40}-46_{8,39}$ & 259016.319 & 6 & 331 & 156.5 & 1609 & Blend with $\mathrm{H}^{13} \mathrm{CN}$ in absorption and $\mathrm{CH}_{3} \mathrm{CN}, v_{8}=2$ \\
\hline 674 & $96_{11,85}-96_{10,86}$ & 259022.243 & 75 & 1306 & 29.4 & 1609 & $\begin{array}{l}\text { Blend with } \mathrm{H}^{13} \mathrm{CN} \text { in absorption, } \mathrm{CH}_{3} \mathrm{CN}, v_{8}=2 \text {, and } \\
\mathrm{CH}_{3}{ }^{13} \mathrm{CH}_{2} \mathrm{CN}\end{array}$ \\
\hline 675 & $47_{8,39}-46_{8,38}$ & 259024.824 & 6 & 331 & 156.5 & 1609 & $\begin{array}{l}\text { Blend with } \mathrm{H}^{13} \mathrm{CN} \text { in absorption, } \mathrm{CH}_{3} \mathrm{CN}, v_{8}=2 \text {, and } \\
\mathrm{CH}_{3}{ }^{13} \mathrm{CH}_{2} \mathrm{CN}\end{array}$ \\
\hline 676 & $94_{7,87}-94_{6,88}$ & 259169.651 & 93 & 1215 & 24.0 & 1609 & Noisy, blend with $\mathrm{CH}_{3} \mathrm{CH}_{3} \mathrm{CO}, v_{t}=1$ and $\mathrm{C}_{2} \mathrm{H}_{5} \mathrm{CN}, v_{13}=1 / v_{21}=1$ \\
\hline 677 & $49_{1,48}-48_{2,47}$ & 259170.075 & 6 & 306 & 18.6 & 1609 & Noisy, blend with $\mathrm{CH}_{3} \mathrm{CH}_{3} \mathrm{CO}, v_{t}=1$ and $\mathrm{C}_{2} \mathrm{H}_{5} \mathrm{CN}, v_{13}=1 / v_{21}=1$ \\
\hline 678 & $49_{2,48}-48_{2,47}$ & 259226.397 & 6 & 306 & 167.4 & 1609 & Strong $\mathrm{C}_{2} \mathrm{H}_{5}{ }^{13} \mathrm{CN},{ }^{13} \mathrm{CH}_{2} \mathrm{CHCN}$, and $\mathrm{C}_{2} \mathrm{H}_{5} \mathrm{CN}$ \\
\hline 679 & $49_{1,48}-48_{1,47}$ & 259241.389 & 6 & 306 & 167.4 & 1609 & Strong $\mathrm{C}_{2} \mathrm{H}_{5}{ }^{13} \mathrm{CN},{ }^{13} \mathrm{CH}_{2} \mathrm{CHCN}$, and $\mathrm{C}_{2} \mathrm{H}_{5} \mathrm{CN}$ \\
\hline 680 & $75_{4,71}-75_{3,72}$ & 259244.206 & 58 & 756 & 13.4 & 1609 & Strong $\mathrm{C}_{2} \mathrm{H}_{5}{ }^{13} \mathrm{CN},{ }^{13} \mathrm{CH}_{2} \mathrm{CHCN}$, and $\mathrm{C}_{2} \mathrm{H}_{5} \mathrm{CN}$ \\
\hline 681 & $48_{3,46}-47_{2,45}$ & 260229.609 & 6 & 302 & 14.3 & 413 & Strong $\mathrm{C}_{2} \mathrm{H}_{5} \mathrm{CN}$ \\
\hline 682 & $23_{5,18}-22_{4,19}$ & 260510.203 & 11 & 78 & 3.6 & 413 & Strong $\mathrm{C}_{2} \mathrm{H}_{5} \mathrm{CN}, v_{13}=1 / v_{21}=1$ \\
\hline 683 & $48_{20,28}-47_{20,27}$ & 263352.009 & 12 & 585 & 136.1 & 74 & Blend with $\mathrm{HCC}^{13} \mathrm{CN}, v_{7}=1$ and ${ }^{13} \mathrm{CH}_{3} \mathrm{C}$ \\
\hline 685 & $48_{19,29}-47_{19,28}$ * & 263368.180 & 9 & 557 & 138.9 & 74 & Blend with $\mathrm{NH}_{2} \mathrm{CH}_{2} \mathrm{CN}$ \\
\hline 687 & $48_{18,30}-47_{18,29}$ * & 263389.051 & 7 & 530 & 141.5 & 74 & Strong $\mathrm{HNCO}, v_{4}=1$ and $\mathrm{CH}_{3} \mathrm{OCH}_{3}$ \\
\hline 689 & $48_{17,31}-47_{17,30}$ * & 263415.803 & 7 & 505 & 144.0 & 74 & Strong $\mathrm{CH}_{3} \mathrm{OCH}_{3}$ \\
\hline 691 & $48_{16,32}-47_{16,31}$ * & 263449.974 & 6 & 481 & 146.4 & 74 & Strong HNCO \\
\hline 693 & $48_{15,33}-47_{15,32}{ }^{\star}$ & 263493.569 & 100 & 459 & 148.6 & 74 & Blend with $\mathrm{C}_{2} \mathrm{H}_{5}{ }^{13} \mathrm{CN}$ and $\mathrm{CH}_{3} \mathrm{OCH}_{3}$ \\
\hline 695 & $48_{14,34}-47_{14,33}{ }^{\star}$ & 263549.541 & 100 & 438 & 150.6 & 74 & Strong $\mathrm{NH}_{2} \mathrm{CHO}, \mathrm{SO}_{2}$, and $\mathrm{NH}_{2} \mathrm{CHO}, v_{12}=1$ \\
\hline 697 & $18_{6,13}-17_{5,12}$ & 263612.153 & 12 & 58 & 3.6 & 74 & Blend with $\mathrm{NH}_{2} \mathrm{CH}_{2} \mathrm{CN}$ and $\mathrm{CH}_{3} \mathrm{OCH}_{3}$ \\
\hline 698 & $18_{6,12}-17_{5,13}$ & 263614.442 & 12 & 58 & 3.6 & 74 & Blend with $\mathrm{NH}_{2} \mathrm{CH}_{2} \mathrm{CN}$ and $\mathrm{CH}_{3} \mathrm{OCH}_{3}$ \\
\hline 699 & $48_{13,35}-47_{13,34}$ & 263621.701 & 6 & 419 & 152.5 & 74 & Blend with $\mathrm{CH}_{3} \mathrm{OCH}_{3}$ \\
\hline 700 & $48_{13,36}-47_{13,35}$ & 263621.895 & 100 & 419 & 152.5 & 74 & Blend with $\mathrm{CH}_{3} \mathrm{OCH}_{3}$ \\
\hline 701 & $49_{3,47}-48_{3,46}$ & 263687.072 & 100 & 314 & 167.0 & 108 & Strong $\mathrm{HNCO}$ and $\mathrm{HN}^{13} \mathrm{CO}$ \\
\hline 702 & $48_{12,36}-47_{12,35}{ }^{\star}$ & 263715.805 & 6 & 401 & 154.4 & 108 & Blend with $\mathrm{HCC}^{13} \mathrm{CN}, v_{7}=1$ and $\mathrm{HNCO}, v_{6}=1$ \\
\hline 704 & $47_{5,42}-46_{5,41}$ & 263828.012 & 6 & 305 & 159.5 & 108 & Blend with $\mathrm{HC}_{3} \mathrm{~N}, v_{5}=1 / v_{7}=3$ and $\mathrm{C}_{2} \mathrm{H}_{5} \mathrm{CN}$ \\
\hline 705 & $48_{11,38}-47_{11,37}{ }^{\star}$ & 263840.460 & 6 & 384 & 156.0 & 108 & Blend with $U$-line and $\mathrm{NH}_{2} \mathrm{CHO}$ \\
\hline 707 & $49_{2,47}-48_{2,46}$ & 263943.595 & 6 & 314 & 167.0 & 108 & Blend with $U$-line and $\mathrm{NH}_{2} \mathrm{CHO}$ \\
\hline 708 & $76_{4,72}-76_{3,73}$ & 264005.376 & 63 & 775 & 13.4 & 108 & Strong $\mathrm{CH}_{3}{ }^{13} \mathrm{CH}_{2} \mathrm{CN}$ and $\mathrm{C}_{2} \mathrm{H}_{5} \mathrm{CN}$ \\
\hline 709 & $48_{10,39}-47_{10,38}{ }^{\star}$ & 264009.192 & 6 & 369 & 157.5 & 108 & Strong $\mathrm{CH}_{3}{ }^{13} \mathrm{CH}_{2} \mathrm{CN}$ and $\mathrm{C}_{2} \mathrm{H}_{5} \mathrm{CN}$ \\
\hline 711 & $48_{6,42}-47_{6,41}$ & 266696.771 & 100 & 324 & 162.1 & 91 & Strong $\mathrm{CH}_{3}{ }^{13} \mathrm{CH}_{2} \mathrm{CN}, \mathrm{C}_{2} \mathrm{H}_{5} \mathrm{CN}$, and $\mathrm{CH}_{3} \mathrm{OH}$ \\
\hline
\end{tabular}

Notes: ${ }^{a}$ Numbering of the observed transitions associated with a modeled line stronger than $20 \mathrm{mK} .{ }^{b}$ Transitions marked with a ${ }^{\star}$ are double with a frequency difference less than $0.1 \mathrm{MHz}$. The quantum numbers of the second one are not shown. ${ }^{c}$ Frequency uncertainty. ${ }^{d}$ Lower energy level in temperature units $\left(E_{\mathrm{l}} / k_{\mathrm{B}}\right) .{ }^{e}$ Calculated rms noise level in $T_{\mathrm{mb}}$ scale. 
A. Belloche et al.: Detection and chemical modeling of ethyl formate and $n$-propyl cyanide in Sgr B2(N), Online Material $p 9$

Table 2. Transitions of the gauche-conformer of ethyl formate observed with the IRAM $30 \mathrm{~m}$ telescope toward Sgr B2(N). The horizontal lines mark discontinuities in the observed frequency coverage. Only the transitions associated with a modeled line stronger than $20 \mathrm{mK}$ are listed.

\begin{tabular}{|c|c|c|c|c|c|c|c|}
\hline$\overline{N^{a}}$ & $\begin{array}{c}\text { Transition }^{b} \\
\text { (2) }\end{array}$ & $\begin{array}{c}\text { Frequency } \\
(\mathrm{MHz}) \\
(3)\end{array}$ & $\begin{array}{c}\text { Unc. }^{c} \\
(\mathrm{kHz}) \\
(4)\end{array}$ & $\begin{array}{l}E_{1}^{d} \\
(\mathrm{~K}) \\
(5)\end{array}$ & $\begin{array}{c}S \mu^{2} \\
\left(\mathrm{D}^{2}\right) \\
(6)\end{array}$ & $\begin{array}{c}\sigma^{e} \\
(\mathrm{mK}) \\
(7)\end{array}$ & $\begin{array}{c}\text { Comments } \\
\text { (8) }\end{array}$ \\
\hline 1 & $14_{7,8}-13_{7,7}$ & 99252.267 & 9 & 140 & 43.9 & 19 & Blend with $\mathrm{a}\left(\mathrm{CH}_{2} \mathrm{OH}\right)_{2}$ and $\mathrm{C}_{2} \mathrm{H}_{5} \mathrm{CN}$ \\
\hline 2 & $14_{7,7}-13_{7,6}$ & 99252.460 & 9 & 140 & 43.9 & 19 & Blend with $\mathrm{a}\left(\mathrm{CH}_{2} \mathrm{OH}\right)_{2}$ and $\mathrm{C}_{2} \mathrm{H}_{5} \mathrm{CN}$ \\
\hline 3 & $16_{1,16}-15_{1,15}$ & 104834.473 & 10 & 132 & 66.3 & 25 & Blend with $\mathrm{C}_{2} \mathrm{H}_{5} \mathrm{CN}, v_{13}=1 / v_{21}=1$ and $U$-line \\
\hline 4 & $16_{0,16}-15_{0,15}$ & 104848.839 & 10 & 132 & 66.3 & 25 & Blend with $\mathrm{C}_{2} \mathrm{H}_{3} \mathrm{CN}, v_{11}=1 / v_{15}=1$ \\
\hline 5 & $15_{9,7}-14_{9,6}^{\star}$ & 106216.238 & 9 & 154 & 40.2 & 25 & Blend with $\mathrm{C}_{2} \mathrm{H}_{3} \mathrm{CN}, v_{11}=1 / v_{15}=1$ \\
\hline 7 & $15_{8,8}-14_{8,7}{ }^{\star}$ & 106286.532 & 9 & 149 & 44.9 & 25 & Blend with $U$-line \\
\hline 9 & $15_{7,9}^{, 0}-14_{7,8}^{\circ, r}$ & 106400.093 & 9 & 144 & 49.1 & 25 & Blend with $U$-line \\
\hline 10 & $15_{7,8}-14_{7,7}$ & 106400.593 & 9 & 144 & 49.1 & 25 & Blend with $U$-line \\
\hline 11 & $15_{2,13}-14_{2,12}$ & 107933.484 & 100 & 131 & 60.9 & 46 & Blend with $\mathrm{C}_{2} \mathrm{H}_{5} \mathrm{CN}, v_{13}=1 / v_{21}=1, \mathrm{C}_{3} \mathrm{H}_{7} \mathrm{CN}$, and $U$-line \\
\hline 12 & $15_{4,11}-14_{4,10}$ & 108497.732 & 10 & 134 & 58.3 & 20 & Blend with $\mathrm{C}_{2} \mathrm{H}_{5} \mathrm{CN}$ and $U$-line \\
\hline 13 & $16_{2,15}-15_{2,14}$ & 108858.373 & 9 & 135 & 65.2 & 20 & Blend with ${ }^{13} \mathrm{CN}$ in absorption, $\mathrm{CH}_{3} \mathrm{CH}_{3} \mathrm{CO}, v_{t}=1$, and $U$-line \\
\hline 14 & $16_{1,15}-15_{1,14}$ & 109206.256 & 100 & 135 & 65.3 & 29 & Blend with $\mathrm{C}_{2} \mathrm{H}_{5} \mathrm{CN}, v_{13}=1 / v_{21}=1$ and $U$-line \\
\hline 15 & $15_{3,12}-14_{3,11}$ & 110117.142 & 10 & 133 & 60.4 & 24 & Blend with $U$-lines \\
\hline 16 & $17_{1,17}-16_{1,16}$ & 111255.183 & 100 & 137 & 70.5 & 35 & Blend with $\mathrm{CH}_{3} \mathrm{OH}$ \\
\hline 17 & $17_{0,17}-16_{0,16}$ & 111263.830 & 100 & 137 & 70.5 & 35 & Blend with $\mathrm{C}_{2} \mathrm{H}_{3} \mathrm{CN}, v_{11}=1 / v_{15}=1$ and ${ }^{13} \mathrm{CH}_{3} \mathrm{CH}_{2} \mathrm{CN}$ \\
\hline 18 & $16_{3,14}-15_{3,13}$ & 112186.512 & 8 & 137 & 64.2 & 42 & Blend with $\mathrm{CH}_{2}{ }^{13} \mathrm{CHCN}$ \\
\hline 19 & $16_{10,6}-15_{10,5}$ * & 113276.855 & 9 & 165 & 40.8 & 28 & Blend with $\mathrm{CH}_{3} \mathrm{OCH}_{3}$ \\
\hline 21 & $16_{9,8}-15_{9,7} \star$ & 113332.031 & 9 & 159 & 45.8 & 28 & Blend with $\mathrm{C}_{2} \mathrm{H}_{5} \mathrm{CN}, v_{13}=1 / v_{21}=1$ and $\mathrm{CH}_{2}{ }^{13} \mathrm{CHCN}$ \\
\hline 23 & $16_{8,9}-15_{8,8}{ }^{\star}$ & 113419.845 & 9 & 154 & 50.2 & 28 & Blend with $\mathrm{C}_{2} \mathrm{H}_{3} \mathrm{CN}, v_{11}=1$ \\
\hline 25 & $46_{10,37}-46_{9,38}$ & 113560.008 & 23 & 488 & 62.8 & 28 & Strong $\mathrm{CN}$ in absorption \\
\hline 26 & $16_{7,10}-15_{7,9}$ & 113560.122 & 9 & 150 & 54.1 & 28 & Strong $\mathrm{CN}$ in absorption \\
\hline 27 & $16_{7,9}-15_{7,8}$ & 113561.324 & 9 & 150 & 54.1 & 28 & Strong CN in absorption \\
\hline 28 & $16_{6,11}-15_{6,10}$ & 113783.618 & 9 & 146 & 57.5 & 34 & Blend with $U$-line \\
\hline 29 & $16_{6,10}-15_{6,9}$ & 113810.530 & 9 & 146 & 57.5 & 34 & Blend with $\mathrm{C}_{2} \mathrm{H}_{3} \mathrm{CN}, v_{15}=1$ \\
\hline 30 & $16_{4,13}-15_{4,12}$ & 113866.411 & 9 & 139 & 62.7 & 34 & Blend with $\mathrm{a}-\mathrm{C}_{2} \mathrm{H}_{5} \mathrm{OCHO}$ and $U$-line \\
\hline 31 & $16_{5,12}-15_{5,11}$ & 114054.354 & 9 & 142 & 60.4 & 33 & Blend with $\mathrm{C}_{2} \mathrm{H}_{3} \mathrm{CN}, v_{15}=1$ \\
\hline 32 & $16_{2,14}-15_{2,13}$ & 114356.265 & 100 & 137 & 64.9 & 33 & Blend with $\mathrm{CH}_{3} \mathrm{CH}_{3} \mathrm{CO}$ and $U$-line \\
\hline 33 & $16_{5,11}-15_{5,10}$ & 114420.852 & 100 & 142 & 60.4 & 37 & Blend with $\mathrm{C}_{2} \mathrm{H}_{3} \mathrm{CN}, v_{11}=1 / v_{15}=1$ \\
\hline 34 & $42_{6,36}-42_{5,37}$ & 115318.413 & 41 & 411 & 44.6 & 60 & Strong CO \\
\hline 35 & $17_{2,16}-16_{2,15}$ & 115319.089 & 9 & 140 & 69.4 & 60 & Strong CO \\
\hline 36 & $17_{1,16}-16_{1,15}$ & 115551.954 & 100 & 140 & 69.4 & 60 & Blend with $\mathrm{C}_{2} \mathrm{H}_{3} \mathrm{CN}$ \\
\hline 37 & $22_{1,21}-21_{2,20}$ & 147415.492 & 100 & 171 & 37.3 & 31 & Blend with $\mathrm{CH}_{3} \mathrm{CH}_{3} \mathrm{CO}, v_{t}=1$ and $\mathrm{CH}_{3}{ }^{13} \mathrm{CN}, v_{8}=1$ \\
\hline 38 & $9_{7,3}-8_{6,2}^{\star}$ & 147431.313 & 18 & 117 & 14.5 & 31 & Blend with $\mathrm{C}_{2} \mathrm{H}_{5} \mathrm{OH}$ and $\mathrm{CH}_{3} \mathrm{CN}, v_{8}=1$ \\
\hline 40 & $9,3-8_{6,3}{ }^{\star}$ & 147431.321 & 18 & 117 & 0.9 & 31 & Blend with $\mathrm{C}_{2} \mathrm{H}_{5} \mathrm{OH}$ and $\mathrm{CH}_{3} \mathrm{CN}, v_{8}=1$ \\
\hline 42 & $22_{2,21}-21_{2,20}$ & 147454.440 & 100 & 171 & 90.3 & 31 & Blend with $\mathrm{a}\left(\mathrm{CH}_{2} \mathrm{OH}\right)_{2}, \mathrm{CH}_{3}{ }^{13} \mathrm{CN}, v_{8}=1$, and $\mathrm{CH}_{3} \mathrm{OCH}_{3}$ \\
\hline 43 & $22_{1,21}-21_{1,20}$ & 147479.064 & 100 & 171 & 90.3 & 31 & Blend with $\mathrm{CH}_{3} \mathrm{CN}, v_{8}=1$ and ${ }^{13} \mathrm{CH}_{3} \mathrm{CH}_{2} \mathrm{CN}$ \\
\hline 44 & $22_{2,21}-21_{1,20}$ & 147517.966 & 100 & 171 & 37.3 & 31 & Blend with $\mathrm{CH}_{3}{ }^{13} \mathrm{CN}, v_{8}=1$ and $\mathrm{CH}_{3} \mathrm{CN}, v_{8}=1$ \\
\hline 45 & $10_{7,4}-9_{6,3^{\star}}$ & 154495.310 & 18 & 120 & 14.6 & 112 & Blend with $\mathrm{C}_{2} \mathrm{H}_{5} \mathrm{CN}$ \\
\hline 47 & $10_{7,4}-9_{6,4}{ }^{\star}$ & 154495.348 & 18 & 120 & 0.9 & 112 & Blend with $\mathrm{C}_{2} \mathrm{H}_{5} \mathrm{CN}$ \\
\hline 49 & $23_{9,15}-22_{9,14}$ & 163354.580 & 7 & 205 & 81.5 & 38 & $\begin{array}{l}\text { Group possibly detected, partial blend with } \mathrm{c}_{-} \mathrm{C}_{2} \mathrm{H}_{4} \mathrm{O} \text {, uncertain } \\
\text { baseline }\end{array}$ \\
\hline 50 & $23_{9,14}-22_{9,13}$ & 163355.083 & 7 & 205 & 81.5 & 38 & $\begin{array}{l}\text { Group possibly detected, partial blend with } \mathrm{c}_{-} \mathrm{C}_{2} \mathrm{H}_{4} \mathrm{O} \text {, uncertain } \\
\text { baseline }\end{array}$ \\
\hline 51 & $23_{3,20}-22_{3,19}$ & 163550.748 & 100 & 184 & 93.0 & 38 & Blend with $\mathrm{CH}_{3} \mathrm{OCHO}, v_{\mathrm{t}}=1$ and $\mathrm{CH}_{2} \mathrm{CH}^{13} \mathrm{CN}$ \\
\hline 52 & $23_{8,16}-22_{8,15}$ & 163648.730 & 7 & 199 & 84.6 & 38 & Blend with $\mathrm{C}_{3} \mathrm{H}_{7} \mathrm{CN}$ and $\mathrm{NH}_{2}{ }^{13} \mathrm{CHO}$ \\
\hline 53 & $23_{8,15}-22_{8,14}$ & 163658.516 & 7 & 199 & 84.6 & 38 & Blend with $\mathrm{C}_{3} \mathrm{H}_{7} \mathrm{CN}$, uncertain baseline \\
\hline 54 & $24_{5,19}-23_{5,18}$ & 176014.876 & 9 & 196 & 96.4 & 365 & Blend with $\mathrm{CH}_{3} \mathrm{OCHO}, \mathrm{CH}_{2} \mathrm{CH}^{13} \mathrm{CN}$, and $\mathrm{HNCO}, v_{5}=1$ \\
\hline 55 & $19_{6,14}-18_{5,13}$ & 202061.374 & 15 & 160 & 13.8 & 138 & Blend with $\mathrm{CH}_{3}{ }^{13} \mathrm{CN}$ \\
\hline 56 & $15_{8,8}^{0,14}-14_{7,7}^{\star}$ & 202643.074 & 17 & 144 & 17.5 & 108 & Blend with $\mathrm{CH}_{3} \mathrm{CN}, v_{8}=1$ and $\mathrm{CH}_{3}{ }^{13} \mathrm{CN}, v_{8}=1$ \\
\hline 58 & $15_{8,8}-14_{7,8}{ }^{\star}$ & 202643.365 & 17 & 144 & 1.1 & 108 & Blend with $\mathrm{CH}_{3} \mathrm{CN}, v_{8}=1$ and $\mathrm{CH}_{3}{ }^{13} \mathrm{CN}, v_{8}=1$ \\
\hline 60 & $30_{2,28}-29_{3,27}$ & 202805.197 & 7 & 241 & 47.3 & 138 & Blend with $\mathrm{C}_{2} \mathrm{H}_{5} \mathrm{CN}$ and $\mathrm{CH}_{3}{ }^{13} \mathrm{CN}, v_{8}=1$ \\
\hline 61 & $30_{3,28}-29_{3,27}$ & 202824.924 & 100 & 241 & 122.7 & 138 & Strong $\mathrm{CH}_{3} \mathrm{CN}, v_{8}=1$ \\
\hline 62 & $30_{2,28}-29_{2,27}$ & 202837.051 & 100 & 241 & 122.6 & 138 & Strong $\mathrm{CH}_{3} \mathrm{CN}, v_{8}=1$ \\
\hline 63 & $45_{9,37}-44_{10,34}$ & 202856.742 & 41 & 463 & 10.5 & 138 & Blend with $\mathrm{CH}_{3} \mathrm{CN}, v_{8}=1$ \\
\hline 64 & $30_{3,28}-29_{2,27}$ & 202856.967 & 7 & 241 & 47.3 & 138 & Blend with $\mathrm{CH}_{3} \mathrm{CN}, v_{8}=1$ \\
\hline 65 & $65_{8,58}-65_{7,59}^{2,7}$ & 202857.921 & 214 & 828 & 52.8 & 138 & Blend with $\mathrm{CH}_{3} \mathrm{CN}, v_{8}=1$ \\
\hline 66 & $17_{7,11}-16_{6,10}$ & 203524.096 & 16 & 151 & 15.9 & 161 & Blend with $\mathrm{CH}_{3} \mathrm{CN}, v_{8}=2$ \\
\hline 67 & $17_{7,10}-16_{6,10}$ & 203528.806 & 16 & 151 & 1.1 & 161 & Blend with $\mathrm{CH}_{3} \mathrm{CN}, v_{8}=2$ \\
\hline 68 & $17_{7,11}-16_{6,11}$ & 203574.259 & 16 & 151 & 1.1 & 161 & Blend with $\mathrm{CH}_{3} \mathrm{CN}, v_{8}=2$ \\
\hline 69 & $17_{7,10}-16_{6,11}$ & 203578.970 & 16 & 151 & 15.9 & 161 & Blend with $\mathrm{CH}_{3} \mathrm{CN}, v_{8}=2$ and $\mathrm{C}_{2} \mathrm{H}_{5} \mathrm{CN}, v_{20}=1$ \\
\hline 70 & $29_{5,25}-28_{5,24}$ & 204412.101 & 7 & 240 & 116.8 & 316 & Blend with ${ }^{13} \mathrm{CH}_{3} \mathrm{CH}_{2} \mathrm{CN}$ and $U$-lines \\
\hline 71 & $19_{6,14}-18_{5,14}$ & 204417.969 & 16 & 160 & 1.2 & 316 & Blend with ${ }^{13} \mathrm{CH}_{3} \mathrm{CH}_{2} \mathrm{CN}$ and $U$-lines \\
\hline
\end{tabular}


A. Belloche et al.: Detection and chemical modeling of ethyl formate and $n$-propyl cyanide in Sgr B2(N), Online Material $p 10$

Table 2. continued.

\begin{tabular}{|c|c|c|c|c|c|c|c|}
\hline$N^{a}$ & $\begin{array}{c}\text { Transition }^{b} \\
\text { (2) } \\
\end{array}$ & $\begin{array}{c}\text { Frequency } \\
(\mathrm{MHz}) \\
(3) \\
\end{array}$ & $\begin{array}{c}\text { Unc. }^{c} \\
(\mathrm{kHz}) \\
(4) \\
\end{array}$ & $\begin{array}{l}E_{\mathrm{l}}^{d} \\
(\mathrm{~K}) \\
(5) \\
\end{array}$ & $\begin{array}{c}S \mu^{2} \\
\left(\mathrm{D}^{2}\right) \\
(6) \\
\end{array}$ & $\begin{array}{c}\sigma^{e} \\
(\mathrm{mK}) \\
(7) \\
\end{array}$ & $\begin{array}{c}\text { Comments } \\
(8) \\
\end{array}$ \\
\hline 72 & $59_{17,43}-59_{16,44}$ & 205092.813 & 17 & 774 & 72.3 & 100 & Strong $\mathrm{C}_{2} \mathrm{H}_{5} \mathrm{CN}, \mathrm{CH}_{3} \mathrm{OCH}_{3}$, and $\mathrm{CH}_{3}{ }^{13} \mathrm{CH}_{2} \mathrm{CN}$ \\
\hline 73 & $28_{5,23}-27_{5,22}$ & 205093.048 & 8 & 232 & 113.5 & 100 & Strong $\mathrm{C}_{2} \mathrm{H}_{5} \mathrm{CN}, \mathrm{CH}_{3} \mathrm{OCH}_{3}$, and $\mathrm{CH}_{3}{ }^{13} \mathrm{CH}_{2} \mathrm{CN}$ \\
\hline 74 & $31_{1,30}-30_{2,29}$ & 205095.436 & 11 & 245 & 57.4 & 100 & Strong $\mathrm{CH}_{3}{ }^{13} \mathrm{CH}_{2} \mathrm{CN}$ and $\mathrm{CH}_{3} \mathrm{OCH}_{3}$ \\
\hline 75 & $31_{2,30}-30_{2,29}$ & 205095.823 & 11 & 245 & 127.9 & 100 & Strong $\mathrm{CH}_{3}{ }^{13} \mathrm{CH}_{2} \mathrm{CN}$ and $\mathrm{CH}_{3} \mathrm{OCH}_{3}$ \\
\hline 76 & $31_{1,30}-30_{1,29}$ & 205096.091 & 11 & 245 & 127.9 & 100 & Strong $\mathrm{CH}_{3}{ }^{13} \mathrm{CH}_{2} \mathrm{CN}$ and $\mathrm{CH}_{3} \mathrm{OCH}_{3}$ \\
\hline 77 & $31_{2,30}-30_{1,29}$ & 205096.478 & 11 & 245 & 57.4 & 100 & Strong $\mathrm{CH}_{3}{ }^{13} \mathrm{CH}_{2} \mathrm{CN}$ and $\mathrm{CH}_{3} \mathrm{OCH}_{3}$ \\
\hline 78 & $29_{18,11}-28_{18,10^{\star}}$ & 205308.386 & 8 & 332 & 74.6 & 100 & Strong $\mathrm{SO}_{2}$ \\
\hline 80 & $29_{17,12}-28_{17,11^{\star}}$ & 205313.243 & 8 & 321 & 79.7 & 100 & Strong $\mathrm{SO}_{2}$ and $U$-line? \\
\hline 82 & $54_{14,40}-53_{15,39^{\star}}$ & 205317.032 & 22 & 650 & 15.2 & 100 & Strong $\mathrm{SO}_{2}$ and $U$-line? \\
\hline 84 & $29_{19,11}-28_{19,10}$ & 205317.035 & 9 & 343 & 69.3 & 100 & Strong $\mathrm{SO}_{2}$ and $U$-line? \\
\hline 85 & $29_{16,13}-28_{16,12^{\star}}$ & 205334.455 & 8 & 311 & 84.4 & 100 & Strong $\mathrm{CH}_{3}{ }^{13} \mathrm{CH}_{2} \mathrm{CN}$ \\
\hline 87 & $29_{20,9}-28_{20,8^{\star}}$ & 205337.037 & 9 & 355 & 63.6 & 100 & Strong $\mathrm{CH}_{3}{ }^{13} \mathrm{CH}_{2} \mathrm{CN}$ \\
\hline 89 & $29_{21,8}-28_{21,7}^{\star}$ & 205366.726 & 10 & 368 & 57.7 & 100 & Blend with $\mathrm{C}_{2} \mathrm{H}_{5}{ }^{13} \mathrm{CN}$ \\
\hline 91 & $29_{15,14}-28_{15,13^{\star}}$ & 205375.865 & 8 & 301 & 88.9 & 100 & Strong $\mathrm{C}_{2} \mathrm{H}_{5} \mathrm{CN}$ \\
\hline 93 & $29_{14,15}-28_{14,14^{\star}}$ & 205442.791 & 7 & 292 & 93.1 & 100 & Blend with $\mathrm{CH}_{3}{ }^{13} \mathrm{CH}_{2} \mathrm{CN}$ and $\mathrm{C}_{2} \mathrm{H}_{5}{ }^{13} \mathrm{CN}$ \\
\hline 95 & $29_{13,17}-28_{13,16^{\star}}$ & 205542.814 & 7 & 284 & 97.0 & 271 & Blend with $U$-line \\
\hline 97 & $29_{12,18}-28_{12,17^{\star}}$ & 205687.132 & 7 & 276 & 100.6 & 271 & Blend with $\mathrm{C}_{2} \mathrm{H}_{3} \mathrm{CN}, v_{11}=1$, uncertain baseline \\
\hline 99 & $29_{27,2}-28_{27,1}{ }^{\star}$ & 205690.300 & 20 & 456 & 16.2 & 271 & Blend with $\mathrm{C}_{2} \mathrm{H}_{3} \mathrm{CN}, v_{11}=1$, uncertain baseline \\
\hline 101 & $29_{11,19}-28_{11,18^{\star}}$ & 205893.033 & 7 & 269 & 103.9 & 271 & Blend with $\mathrm{C}_{2} \mathrm{H}_{5}{ }^{13} \mathrm{CN}$ \\
\hline 103 & $29_{10,20}-28_{10,19}$ & 206188.338 & 7 & 263 & 106.9 & 280 & Blend with $\mathrm{SO}$ and ${ }^{13} \mathrm{CH}_{2} \mathrm{CHCN}$ \\
\hline 104 & $29_{10,19}-28_{10,18}$ & 206190.002 & 7 & 263 & 106.9 & 280 & Blend with $\mathrm{SO}$ and ${ }^{13} \mathrm{CH}_{2} \mathrm{CHCN}$ \\
\hline 105 & $29_{4,25}-28_{4,24}$ & 206280.705 & 100 & 240 & 117.1 & 280 & Blend with $\mathrm{C}_{2} \mathrm{H}_{5} \mathrm{CN}$ and $\mathrm{C}_{2} \mathrm{H}_{5} \mathrm{CN}, v_{20}=1$ \\
\hline 106 & $29_{9,21}-28_{9,20}$ & 206614.666 & 7 & 257 & 109.6 & 106 & Strong $\mathrm{C}_{2} \mathrm{H}_{5} \mathrm{CN}$ and $\mathrm{CH}_{3} \mathrm{OCHO}$ \\
\hline 107 & $29_{9,20}-28_{9,19}$ & 206639.557 & 7 & 257 & 109.6 & 106 & Blend with $U$-lines \\
\hline 108 & $56_{17,40}-56_{16,40}$ & 206650.461 & 14 & 715 & 2.7 & 106 & Blend with $\mathrm{C}_{2} \mathrm{H}_{5} \mathrm{CN}$ \\
\hline 109 & $56_{17,39}-56_{16,40}$ & 206650.601 & 14 & 715 & 67.2 & 106 & Blend with $\mathrm{C}_{2} \mathrm{H}_{5} \mathrm{CN}$ \\
\hline 110 & $56_{17,40}-56_{16,41}$ & 206652.471 & 14 & 715 & 67.2 & 106 & Blend with $\mathrm{C}_{2} \mathrm{H}_{5} \mathrm{CN}$ \\
\hline 111 & $56_{17,39}-56_{16,41}$ & 206652.612 & 14 & 715 & 2.7 & 106 & Blend with $\mathrm{C}_{2} \mathrm{H}_{5} \mathrm{CN}$ \\
\hline 112 & $30_{3,27}-29_{4,26}$ & 206654.482 & 7 & 246 & 38.8 & 106 & Blend with $\mathrm{C}_{2} \mathrm{H}_{5} \mathrm{CN}$ \\
\hline 113 & $29_{6,24}-28_{6,23}$ & 206929.917 & 8 & 244 & 115.8 & 117 & Strong $\mathrm{C}_{2} \mathrm{H}_{5} \mathrm{CN}, v_{20}=1$ and $\mathrm{C}_{2} \mathrm{H}_{5} \mathrm{CN}$ \\
\hline 114 & $30_{4,27}-29_{4,26}$ & 206996.130 & 100 & 246 & 121.8 & 117 & Blend with OCS, $v_{2}=1$ and $\mathrm{NH}_{2}{ }^{13} \mathrm{CHO}$ \\
\hline 115 & $28_{5,24}-27_{4,23}$ & 207002.205 & 100 & 230 & 24.1 & 117 & Blend with OCS, $v_{2}=1$ and $\mathrm{NH}_{2}{ }^{13} \mathrm{CHO}$ \\
\hline 116 & $30_{3,27}-29_{3,26}$ & 207175.063 & 100 & 246 & 121.8 & 117 & Blend with $U$-line \\
\hline 117 & $29_{8,22}-28_{8,21}$ & 207183.249 & 7 & 252 & 112.1 & 117 & Blend with $U$-line \\
\hline 118 & $12_{10,2}-11_{9,3}{ }^{\star}$ & 207240.583 & 16 & 141 & 21.0 & 117 & Blend with $\mathrm{CH}_{3} \mathrm{OCHO}$ and $U$-line \\
\hline 120 & $12_{10,2}-11_{9,2}^{\star}$ & 207240.583 & 16 & 141 & 1.2 & 117 & Blend with $\mathrm{CH}_{3} \mathrm{OCHO}$ and $U$-line \\
\hline 122 & $32_{0,32}-31_{0,31} \star$ & 207408.086 & 100 & 249 & 133.2 & 282 & Blend with $\mathrm{C}_{2} \mathrm{H}_{5} \mathrm{CN}, v_{13}=1 / v_{21}=1$ \\
\hline 124 & $32_{0,32}-31_{1,31} \star$ & 207408.141 & 17 & 249 & 67.2 & 282 & Blend with $\mathrm{C}_{2} \mathrm{H}_{5} \mathrm{CN}, v_{13}=1 / v_{21}=1$ \\
\hline 126 & $29_{8,21}-28_{8,20}$ & 207451.586 & 7 & 252 & 112.1 & 282 & Blend with $\mathrm{C}_{2} \mathrm{H}_{5} \mathrm{CN}, v_{13}=1 / v_{21}=1$ and $\mathrm{C}_{2} \mathrm{H}_{5} \mathrm{OH}$ \\
\hline 127 & $30_{4,27}-29_{3,26}$ & 207516.619 & 7 & 246 & 38.8 & 282 & Blend with $\mathrm{CH}_{3} \mathrm{CH}_{3} \mathrm{CO}, v_{t}=1$ and $U$-line \\
\hline 128 & $29_{7,23}-28_{7,22}$ & 207601.807 & 8 & 247 & 114.2 & 282 & Strong $\mathrm{NH}_{2} \mathrm{CHO}, v_{12}=1$ and $\mathrm{C}_{2} \mathrm{H}_{3} \mathrm{CN}$ \\
\hline 129 & $20_{6,15}-19_{5,14}$ & 207703.938 & 15 & 166 & 13.7 & 282 & Blend with $\mathrm{CH}_{3} \mathrm{OCH}_{3}$ \\
\hline 130 & $14_{9,6}-13_{8,5}{ }^{\star}$ & 208513.304 & 16 & 144 & 19.3 & 168 & Blend with $\mathrm{C}_{2} \mathrm{H}_{5} \mathrm{OH}$ and $\mathrm{C}_{2} \mathrm{H}_{3} \mathrm{CN}$ \\
\hline 132 & $14_{9,5}-13_{8,6}{ }^{\star}$ & 208513.306 & 16 & 144 & 19.3 & 168 & Blend with $\mathrm{C}_{2} \mathrm{H}_{5} \mathrm{OH}$ and $\mathrm{C}_{2} \mathrm{H}_{3} \mathrm{CN}$ \\
\hline 134 & $31_{2,29}-30_{3,28}$ & 209205.202 & 8 & 251 & 49.5 & 58 & Strong $\mathrm{H}_{2} \mathrm{CS}$ and $\mathrm{C}_{2} \mathrm{H}_{3} \mathrm{CN}, v_{15}=1$ \\
\hline 135 & $31_{3,29}-30_{3,28}$ & 209217.484 & 100 & 251 & 126.8 & 58 & Strong $\mathrm{C}_{2} \mathrm{H}_{3} \mathrm{CN}, v_{15}=1, \mathrm{CH}_{3} \mathrm{OCHO}$, and $\mathrm{HC}_{3} \mathrm{~N}$ \\
\hline 136 & $31_{2,29}-30_{2,28}$ & 209225.073 & 100 & 251 & 126.8 & 58 & Strong $\mathrm{CH}_{3} \mathrm{OCHO}$ and $\mathrm{HC}_{3} \mathrm{~N}$ \\
\hline 137 & $31_{3,29}-30_{2,28}$ & 209237.276 & 8 & 251 & 49.5 & 58 & Strong $\mathrm{HC}_{3} \mathrm{~N}$ \\
\hline 138 & $74_{18,56}-74_{17,57^{\star}}$ & 209239.716 & 197 & 1125 & 97.1 & 58 & Strong $\mathrm{HC}_{3} \mathrm{~N}$ \\
\hline 140 & $29_{7,22}-28_{7,21}$ & 209426.660 & 9 & 248 & 114.3 & 58 & Strong $\mathrm{CH}_{3} \mathrm{OCH}_{3}$ and $\mathrm{CH}_{3} \mathrm{CH}_{3} \mathrm{CO}$ \\
\hline 141 & $16_{8,9}-15_{7,8^{\star}}^{\star}$ & 209662.326 & 16 & 150 & 17.7 & 45 & Strong $\mathrm{C}_{2} \mathrm{H}_{3} \mathrm{CN}, v_{11}=1, \mathrm{HC}^{13} \mathrm{CCN}, v_{7}=2$, and $\mathrm{HCC}^{13} \mathrm{CN}, v_{7}=2$ \\
\hline 143 & $16_{8,9}-15_{7,9}{ }^{\star}$ & 209663.117 & 16 & 150 & 1.2 & 45 & Strong $\mathrm{C}_{2} \mathrm{H}_{3} \mathrm{CN}, v_{11}=1, \mathrm{HC}^{13} \mathrm{CCN}, v_{7}=2$, and $\mathrm{HCC}^{13} \mathrm{CN}, v_{7}=2$ \\
\hline 145 & $18_{7,12}-17_{6,11}$ & 210394.041 & 16 & 157 & 16.0 & 64 & Blend with $\mathrm{C}_{2} \mathrm{H}_{5} \mathrm{OH}$ and $\mathrm{CH}_{3} \mathrm{OCHO}$ \\
\hline 146 & $18_{7,11}-17_{6,12}$ & 210507.031 & 16 & 157 & 16.0 & 64 & Strong $\mathrm{HC}_{3} \mathrm{~N}, v_{7}=2$ \\
\hline 147 & $41_{17,24}-41_{16,25}^{\star}$ & 210945.438 & 10 & 465 & 43.1 & 37 & Strong $\mathrm{NH}_{2}{ }^{13} \mathrm{CHO}$ and $\mathrm{C}_{2} \mathrm{H}_{5} \mathrm{CN}, v_{13}=1 / v_{21}=1$ \\
\hline 149 & $41_{17,25}-41_{16,26}^{\star}$ & 210945.438 & 10 & 465 & 43.1 & 37 & Strong $\mathrm{NH}_{2}{ }^{13} \mathrm{CHO}$ and $\mathrm{C}_{2} \mathrm{H}_{5} \mathrm{CN}, v_{13}=1 / v_{21}=1$ \\
\hline 151 & $30_{5,26}-29_{5,25}$ & 210948.194 & 7 & 250 & 121.0 & 37 & Strong $\mathrm{NH}_{2}{ }^{13} \mathrm{CHO}$ and $\mathrm{C}_{2} \mathrm{H}_{5} \mathrm{CN}, v_{13}=1 / v_{21}=1$ \\
\hline 152 & $29_{5,25}-28_{4,24}$ & 211213.126 & 100 & 240 & 26.6 & 33 & Strong $\mathrm{H}_{2} \mathrm{CO}$ in absorption \\
\hline 153 & $32_{1,31}-31_{2,30}$ & 211492.742 & 12 & 255 & 59.6 & 33 & Strong $\mathrm{CH}_{3} \mathrm{OCH}_{3}$ \\
\hline 154 & $32_{2,31}-31_{2,30}$ & 211492.971 & 12 & 255 & 132.0 & 33 & Strong $\mathrm{CH}_{3} \mathrm{OCH}_{3}$ \\
\hline 155 & $32_{1,31}-31_{1,30}$ & 211493.129 & 12 & 255 & 132.0 & 33 & Strong $\mathrm{CH}_{3} \mathrm{OCH}_{3}$ \\
\hline 156 & $32_{2,31}-31_{1,30}$ & 211493.358 & 12 & 255 & 59.6 & 33 & Strong $\mathrm{CH}_{3} \mathrm{OCH}_{3}$ \\
\hline 157 & $29_{5,24}-28_{5,23}$ & 211817.880 & 100 & 242 & 117.5 & 47 & Blend with $\mathrm{CH}_{3} \mathrm{OH}, v_{t}=1$ and $U$-line \\
\hline
\end{tabular}


A. Belloche et al.: Detection and chemical modeling of ethyl formate and $n$-propyl cyanide in Sgr B2(N), Online Material $p 11$

Table 2. continued.

\begin{tabular}{|c|c|c|c|c|c|c|c|}
\hline$N^{a}$ & Transition $^{b}$ & $\begin{array}{c}\text { Frequency } \\
\text { (MHz) } \\
\text { (3) }\end{array}$ & $\begin{array}{l}\text { Unc. }^{c} \\
(\mathrm{kHz}) \\
(4)\end{array}$ & $\begin{array}{l}E_{1}^{d} \\
(\mathrm{~K}) \\
(5)\end{array}$ & $\begin{array}{l}S \mu^{2} \\
\left(\mathrm{D}^{2}\right) \\
(6)\end{array}$ & $\begin{array}{c}\sigma^{e} \\
(\mathrm{mK}) \\
(7)\end{array}$ & Comments \\
\hline 158 & $18_{17,1}-18_{16,2}^{\star}$ & 211857.177 & 16 & 231 & 4.4 & 47 & Blend with $\mathrm{C}_{2} \mathrm{H}_{3} \mathrm{CN}, v_{15}=1$ and $U$-line \\
\hline 160 & $29_{17,12}-29_{16,13^{\star}}$ & 211859.692 & 10 & 320 & 24.2 & 47 & Blend with $\mathrm{C}_{2} \mathrm{H}_{3} \mathrm{CN}, v_{15}=1$ and $U$-line \\
\hline 162 & $29_{17,12}-29_{16,14^{\star}}$ & 211859.692 & 10 & 320 & 1.2 & 47 & Blend with $\mathrm{C}_{2} \mathrm{H}_{3} \mathrm{CN}, v_{15}=1$ and $U$-line \\
\hline 164 & $28_{17,11}-28_{16,12^{\star}}$ & 211880.904 & 10 & 311 & 22.6 & 47 & Blend with $\mathrm{CH}_{2} \mathrm{CH}^{13} \mathrm{CN}$ \\
\hline 166 & $28_{17,11}-28_{16,13^{\star}}$ & 211880.904 & 10 & 311 & 1.1 & 47 & Blend with $\mathrm{CH}_{2} \mathrm{CH}^{13} \mathrm{CN}$ \\
\hline 168 & $27_{17,10}-27_{16,11}$ * & 211896.387 & 11 & 301 & 20.9 & 47 & Strong $\mathrm{C}_{2} \mathrm{H}_{3} \mathrm{CN}, v_{11}=1$ and $\mathrm{C}_{2} \mathrm{H}_{5} \mathrm{OH}$ \\
\hline 170 & $27_{17,10}-27_{16,12^{\star}}$ & 211896.387 & 11 & 301 & 1.0 & 47 & Strong $\mathrm{C}_{2} \mathrm{H}_{3} \mathrm{CN}, v_{11}=1$ and $\mathrm{C}_{2} \mathrm{H}_{5} \mathrm{OH}$ \\
\hline 172 & $21_{17,4}-21_{16,5^{\star}}$ & 211896.595 & 13 & 251 & 10.4 & 47 & Strong $\mathrm{C}_{2} \mathrm{H}_{3} \mathrm{CN}, v_{11}=1$ and $\mathrm{C}_{2} \mathrm{H}_{5} \mathrm{OH}$ \\
\hline 174 & $21_{17,4}-21_{16,6}{ }^{\star}$ & 211896.595 & 13 & 251 & 0.5 & 47 & Strong $\mathrm{C}_{2} \mathrm{H}_{3} \mathrm{CN}, v_{11}=1$ and $\mathrm{C}_{2} \mathrm{H}_{5} \mathrm{OH}$ \\
\hline 176 & $22_{17,5}-22_{16,6}^{\star}$ & 211905.280 & 13 & 259 & 12.2 & 47 & Blend with $\mathrm{CH}_{3} \mathrm{CH}_{3} \mathrm{CO}, v_{t}=1, \mathrm{C}_{2} \mathrm{H}_{3} \mathrm{CN}, v_{11}=1$, and $U$-line \\
\hline 178 & $22_{17,5}-22_{16,7}^{\star}$ & 211905.280 & 13 & 259 & 0.6 & 47 & Blend with $\mathrm{CH}_{3} \mathrm{CH}_{3} \mathrm{CO}, v_{t}=1, \mathrm{C}_{2} \mathrm{H}_{3} \mathrm{CN}, v_{11}=1$, and $U$-line \\
\hline 180 & $26_{17,10}-26_{16,11}{ }^{\star}$ & 211906.656 & 11 & 292 & 19.2 & 47 & Blend with $\mathrm{CH}_{3} \mathrm{CH}_{3} \mathrm{CO}, v_{t}=1, \mathrm{C}_{2} \mathrm{H}_{3} \mathrm{CN}, v_{11}=1$, and $U$-line \\
\hline 182 & $26_{17,10}-26_{16,10}$ & 211906.656 & 11 & 292 & 0.9 & 47 & Blend with $\mathrm{CH}_{3} \mathrm{CH}_{3} \mathrm{CO}, v_{t}=1, \mathrm{C}_{2} \mathrm{H}_{3} \mathrm{CN}, v_{11}=1$, and $U$-line \\
\hline 183 & $26_{17,9}-26_{16,10}$ & 211906.776 & 100 & 292 & 19.2 & 47 & Blend with $\mathrm{CH}_{3} \mathrm{CH}_{3} \mathrm{CO}, v_{t}=1, \mathrm{C}_{2} \mathrm{H}_{3} \mathrm{CN}, v_{11}=1$, and $U$-line \\
\hline 184 & $23_{17,6}-23_{16,7}^{\star}$ & 211911.066 & 12 & 266 & 14.0 & 47 & Blend with $\mathrm{CH}_{3} \mathrm{CH}_{3} \mathrm{CO}, v_{t}=1, \mathrm{C}_{2} \mathrm{H}_{3} \mathrm{CN}, v_{11}=1$, and $U$-line \\
\hline 186 & $23_{17,6}-23_{16,8} \star$ & 211911.066 & 12 & 266 & 0.7 & 47 & Blend with $\mathrm{CH}_{3} \mathrm{CH}_{3} \mathrm{CO}, v_{t}=1, \mathrm{C}_{2} \mathrm{H}_{3} \mathrm{CN}, v_{11}=1$, and $U$-line \\
\hline 188 & $25_{17,8}-25_{16,9}{ }^{\star}$ & 211912.208 & 11 & 283 & 17.5 & 47 & Blend with $\mathrm{CH}_{3} \mathrm{CH}_{3} \mathrm{CO}, v_{t}=1, \mathrm{C}_{2} \mathrm{H}_{3} \mathrm{CN}, v_{11}=1$, and $U$-line \\
\hline 190 & $25_{17,8}-25_{16,10}$ * & 211912.208 & 11 & 283 & 0.9 & 47 & Blend with $\mathrm{CH}_{3} \mathrm{CH}_{3} \mathrm{CO}, v_{t}=1, \mathrm{C}_{2} \mathrm{H}_{3} \mathrm{CN}, v_{11}=1$, and $U$-line \\
\hline 192 & $24_{17,8}-24_{16,9}{ }^{\star}$ & 211913.523 & 12 & 275 & 15.8 & 47 & Blend with $\mathrm{CH}_{3} \mathrm{CH}_{3} \mathrm{CO}, v_{t}=1, \mathrm{C}_{2} \mathrm{H}_{3} \mathrm{CN}, v_{11}=1$, and $U$-line \\
\hline 194 & $24_{17,8}-24_{16,8}$ & 211913.523 & 12 & 275 & 0.8 & 47 & Blend with $\mathrm{CH}_{3} \mathrm{CH}_{3} \mathrm{CO}, v_{t}=1, \mathrm{C}_{2} \mathrm{H}_{3} \mathrm{CN}, v_{11}=1$, and $U$-line \\
\hline 195 & $24_{17,7}-24_{16,8}$ & 211913.626 & 100 & 275 & 15.8 & 47 & Blend with $\mathrm{CH}_{3} \mathrm{CH}_{3} \mathrm{CO}, v_{t}=1, \mathrm{C}_{2} \mathrm{H}_{3} \mathrm{CN}, v_{11}=1$, and $U$-line \\
\hline 196 & $20_{6,14}-19_{5,15}$ & 212146.465 & 17 & 166 & 13.4 & 36 & Strong $\mathrm{NH}_{2} \mathrm{CHO}, v_{12}=1$ \\
\hline 197 & $30_{4,26}-29_{4,25}$ & 212369.981 & 100 & 249 & 121.2 & 36 & $\begin{array}{l}\text { Blend with } U \text {-line, } \mathrm{NH}_{2}{ }^{13} \mathrm{CHO}, v_{12}=1 \text {, and } \mathrm{C}_{3} \mathrm{H}_{7} \mathrm{CN} \text {, uncertain } \\
\text { baseline }\end{array}$ \\
\hline 198 & $30_{18,12}-29_{18,11}{ }^{\star}$ & 212385.195 & 8 & 341 & 80.3 & 36 & Strong $\mathrm{NH}_{2} \mathrm{CHO}, v_{12}=1$ \\
\hline 200 & $71_{18,54}-71_{17,55}$ & 212386.233 & 123 & 1051 & 91.5 & 36 & Strong $\mathrm{NH}_{2} \mathrm{CHO}, v_{12}=1$ \\
\hline 201 & $30_{19,11}-29_{19,10}$ * & 212390.341 & 8 & 353 & 75.2 & 36 & Strong $\mathrm{NH}_{2} \mathrm{CHO}, v_{12}=1$ \\
\hline 203 & $30_{17,13}-29_{17,12}{ }^{\star}$ & 212394.782 & 8 & 331 & 85.2 & 36 & Strong $\mathrm{NH}_{2} \mathrm{CHO}, v_{12}=1$ \\
\hline 205 & $30_{20,11}-29_{20,10}{ }^{\star}$ & 212407.836 & 9 & 365 & 69.7 & 36 & Blend with $\mathrm{NH}_{2}{ }^{13} \mathrm{CHO}, v_{12}=1$ and $\mathrm{CH}_{3} \mathrm{CH}_{3} \mathrm{CO}, v_{t}=1$ \\
\hline 207 & $30_{16,14}-29_{16,13}{ }^{\star}$ & 212422.256 & 8 & 320 & 89.8 & 36 & Strong $\mathrm{NH}_{2} \mathrm{CHO}$ \\
\hline 209 & $30_{21,9}-29_{21,8}{ }^{\star}$ & 212435.843 & 9 & 377 & 64.0 & 36 & Strong $\mathrm{NH}_{2} \mathrm{CHO}$ \\
\hline 211 & $30_{15,15}-29_{15,14^{\star}}$ & 212471.882 & 8 & 311 & 94.1 & 99 & Blend with $\mathrm{C}_{2} \mathrm{H}_{5} \mathrm{OH}, \mathrm{CH}_{3} \mathrm{OCHO}$, and $\mathrm{C}_{3} \mathrm{H}_{7} \mathrm{CN}$ \\
\hline 213 & $30_{22,8}-29_{22,7^{\star}}^{\star}$ & 212472.920 & 10 & 391 & 58.0 & 99 & Blend with $\mathrm{C}_{2} \mathrm{H}_{5} \mathrm{OH}, \mathrm{CH}_{3} \mathrm{OCHO}$, and $\mathrm{C}_{3} \mathrm{H}_{7} \mathrm{CN}$ \\
\hline 215 & $30_{14,17}-29_{14,16}{ }^{\star}$ & 212549.578 & 7 & 302 & 98.2 & 99 & Blend with $\mathrm{NH}_{2}{ }^{13} \mathrm{CHO}$ and $U$-line \\
\hline 217 & $29_{6,23}-28_{6,22}$ & 212550.033 & 10 & 244 & 116.6 & 99 & Blend with $\mathrm{NH}_{2}{ }^{13} \mathrm{CHO}$ and $U$-line \\
\hline 218 & $30_{13,17}-29_{13,16^{\star}}$ & 212663.809 & 7 & 294 & 102.0 & 99 & Blend with $\mathrm{C}_{2} \mathrm{H}_{5} \mathrm{OH}$ and ${ }^{13} \mathrm{CH}_{2} \mathrm{CHCN}$ \\
\hline 220 & $30_{12,19}-29_{12,18^{\star}}$ & 212827.135 & 7 & 286 & 105.4 & 99 & Strong ${ }^{13} \mathrm{CH}_{2} \mathrm{CHCN}$ and $\mathrm{NH}_{2} \mathrm{CHO}$ \\
\hline 222 & $30_{28,3}-29_{28,2^{\star}}$ & 212834.203 & 100 & 482 & 16.2 & 99 & Strong ${ }^{13} \mathrm{CH}_{2} \mathrm{CHCN}$ and $\mathrm{NH}_{2} \mathrm{CHO}$ \\
\hline 224 & $21_{6,16}-20_{5,15}$ & 212845.604 & 15 & 173 & 13.7 & 99 & Blend with ${ }^{13} \mathrm{CH}_{2} \mathrm{CHCN}$, uncertain baseline \\
\hline 225 & $11_{11,1}-10_{10,0} \star$ & 212997.517 & 100 & 143 & 23.2 & 99 & Blend with $\mathrm{C}_{2} \mathrm{H}_{5} \mathrm{CN}$ \\
\hline 227 & $11_{11,0}-10_{10,0}$ * & 212997.572 & 16 & 143 & 1.3 & 99 & Blend with $\mathrm{C}_{2} \mathrm{H}_{5} \mathrm{CN}$ \\
\hline 229 & $30_{11,20}-29_{11,19}$ & 213059.055 & 7 & 279 & 108.7 & 48 & Strong $\mathrm{SO}_{2}$ \\
\hline 230 & $30_{11,19}-29_{11,18}$ & 213059.228 & 7 & 279 & 108.7 & 48 & Strong $\mathrm{SO}_{2}$ \\
\hline 231 & $31_{3,28}-30_{4,27}$ & 213169.983 & 7 & 255 & 41.1 & 48 & Strong $\mathrm{C}_{2} \mathrm{H}_{3} \mathrm{CN}, v_{11}=1$ \\
\hline 232 & $30_{10,21}-29_{10,20}$ & 213390.877 & 7 & 273 & 111.6 & 48 & Blend with $\mathrm{CH}_{3} \mathrm{CH}_{3} \mathrm{CO}, v_{t}=1$, uncertain baseline \\
\hline 233 & $31_{4,28}-30_{4,27}$ & 213392.357 & 7 & 255 & 125.9 & 48 & Blend with $\mathrm{CH}_{3} \mathrm{CH}_{3} \mathrm{CO}, v_{t}=1$, uncertain baseline \\
\hline 234 & $30_{10,20}-29_{10,19}$ & 213394.012 & 7 & 273 & 111.6 & 48 & Blend with $\mathrm{CH}_{3} \mathrm{CH}_{3} \mathrm{CO}, v_{t}=1$, uncertain baseline \\
\hline 235 & $16_{4,13}-15_{1,14}$ & 213509.497 & 41 & 135 & 0.8 & 48 & Blend with $\mathrm{C}_{2} \mathrm{H}_{5} \mathrm{CN}, v_{13}=1 / v_{21}=1$ and $U$-line \\
\hline 236 & $31_{3,28}-30_{3,27}$ & 213511.580 & 100 & 255 & 125.9 & 48 & Blend with $\mathrm{C}_{2} \mathrm{H}_{5} \mathrm{CN}, v_{13}=1 / v_{21}=1$ and $U$-line \\
\hline 237 & $31_{4,28}-30_{3,27}$ & 213733.932 & 7 & 255 & 41.1 & 48 & Blend with $\mathrm{C}_{2} \mathrm{H}_{5} \mathrm{CN}, v_{13}=1 / v_{21}=1$ \\
\hline 238 & $30_{6,25}-29_{6,24}$ & 213791.953 & 100 & 253 & 120.1 & 48 & Blend with $\mathrm{CH}_{3} \mathrm{CH}_{3} \mathrm{CO}, v_{t}=1, \mathrm{C}_{2} \mathrm{H}_{3} \mathrm{CN}, v_{15}=1$, and $U$-line \\
\hline 239 & $33_{0,33}-32_{1,32}{ }^{\star}$ & 213807.575 & 17 & 259 & 69.4 & 48 & Blend with $\mathrm{C}_{2} \mathrm{H}_{5} \mathrm{CN}$ \\
\hline 241 & $33_{0,33}-32_{0,32}{ }^{\star}$ & 213807.621 & 100 & 259 & 137.4 & 48 & Blend with $\mathrm{C}_{2} \mathrm{H}_{5} \mathrm{CN}$ \\
\hline 243 & $30_{9,22}-29_{9,21}$ & 213865.943 & 7 & 267 & 114.2 & 48 & Blend with $\mathrm{C}_{2} \mathrm{H}_{5} \mathrm{OH}$ and $\mathrm{C}_{2} \mathrm{H}_{5}{ }^{13} \mathrm{CN}$ \\
\hline 244 & $30_{9,21}-29_{9,20}$ & 213909.016 & 7 & 267 & 114.2 & 48 & Blend with $\mathrm{C}_{3} \mathrm{H}_{7} \mathrm{CN}$ and ${ }^{13} \mathrm{CH}_{3} \mathrm{OH}$ \\
\hline 245 & $13_{10,3}-12_{9,4}$ * & 214314.472 & 16 & 145 & 21.1 & 75 & Strong ${ }^{13} \mathrm{CH}_{3} \mathrm{CN}$ and $\mathrm{C}_{2} \mathrm{H}_{5}{ }^{13} \mathrm{CN}$ \\
\hline 247 & $13_{10,3}-12_{9,3}{ }^{\star}$ & 214314.472 & 16 & 145 & 1.2 & 75 & Strong ${ }^{13} \mathrm{CH}_{3} \mathrm{CN}$ and $\mathrm{C}_{2} \mathrm{H}_{5}{ }^{13} \mathrm{CN}$ \\
\hline 249 & $30_{8,23}-29_{8,22}$ & 214467.553 & 8 & 262 & 116.6 & 75 & Blend with $\mathrm{CH}_{3} \mathrm{OCH}_{3}$ \\
\hline 250 & $30_{7,24}-29_{7,23}$ & 214783.566 & 8 & 257 & 118.6 & 75 & Strong $\mathrm{CH}_{3} \mathrm{OCHO}$ \\
\hline 251 & $30_{8,22}-29_{8,21}$ & 214889.514 & 8 & 262 & 116.6 & 74 & Blend with ${ }^{13} \mathrm{CH}_{2} \mathrm{CHCN}$ \\
\hline 252 & $31_{4,27}-30_{5,26}$ & 214982.375 & 100 & 260 & 31.4 & 74 & Strong $\mathrm{C}_{2} \mathrm{H}_{5}{ }^{13} \mathrm{CN}$ and ${ }^{13} \mathrm{CH}_{3} \mathrm{CN}, v_{8}=1$ \\
\hline 253 & $15_{9,7}-14_{8,6^{\star}}{ }^{\star}$ & 215567.674 & 16 & 149 & 19.5 & 74 & Strong $\mathrm{C}_{2} \mathrm{H}_{5} \mathrm{CN}, v_{20}=1$ and $\mathrm{C}_{2} \mathrm{H}_{5} \mathrm{CN}, v_{13}=1 / v_{21}=1$ \\
\hline 255 & $15_{9,7}-14_{8,7}{ }^{\star}$ & 215567.680 & 16 & 149 & 1.2 & 74 & Strong $\mathrm{C}_{2} \mathrm{H}_{5} \mathrm{CN}, v_{20}=1$ and $\mathrm{C}_{2} \mathrm{H}_{5} \mathrm{CN}, v_{13}=1 / v_{21}=1$ \\
\hline
\end{tabular}


A. Belloche et al.: Detection and chemical modeling of ethyl formate and $n$-propyl cyanide in Sgr B2(N), Online Material $p 12$

Table 2. continued.

\begin{tabular}{|c|c|c|c|c|c|c|c|}
\hline$\overline{N^{a}}$ & Transition $^{b}$ & $\begin{array}{c}\text { Frequency } \\
(\mathrm{MHz}) \\
(3)\end{array}$ & $\begin{array}{c}\text { Unc. }^{c} \\
(\mathrm{kHz}) \\
(4)\end{array}$ & $\begin{array}{l}E_{1}^{d} \\
(\mathrm{~K}) \\
(5)\end{array}$ & $\begin{array}{l}S \mu^{2} \\
\left(\mathrm{D}^{2}\right) \\
(6)\end{array}$ & $\begin{array}{c}\sigma^{e} \\
(\mathrm{mK}) \\
(7)\end{array}$ & $\begin{array}{c}\text { Comments } \\
\text { (8) }\end{array}$ \\
\hline 257 & $32_{2,30}-31_{3,29}$ & 215600.637 & 8 & 261 & 51.8 & 74 & Strong $\mathrm{C}_{2} \mathrm{H}_{5} \mathrm{CN}$ \\
\hline 258 & $32_{3,30}-31_{3,29}$ & 215608.230 & 100 & 261 & 131.0 & 74 & Strong $\mathrm{C}_{2} \mathrm{H}_{5} \mathrm{CN}$ \\
\hline 259 & $32_{2,30}-31_{2,29}$ & 215612.952 & 100 & 261 & 131.0 & 74 & Strong $\mathrm{C}_{2} \mathrm{H}_{5} \mathrm{CN}$ \\
\hline 260 & $32_{3,30}-31_{2,29}$ & 215620.426 & 8 & 261 & 51.8 & 74 & Strong $\mathrm{C}_{2} \mathrm{H}_{5} \mathrm{CN}$ \\
\hline 261 & $36_{9,27}-36_{6,30}$ & 215623.049 & 44 & 336 & 2.1 & 74 & Strong $\mathrm{C}_{2} \mathrm{H}_{5} \mathrm{CN}$ \\
\hline 262 & $30_{5,26}-29_{4,25}$ & 215880.577 & 100 & 249 & 29.1 & 55 & Strong ${ }^{13} \mathrm{CH}_{3} \mathrm{OH}$ \\
\hline 263 & $17,10-16_{7,9}$ & 216663.426 & 16 & 155 & 17.9 & 55 & Blend with $\mathrm{C}_{2} \mathrm{H}_{5} \mathrm{OH}, \mathrm{C}_{3} \mathrm{H}_{7} \mathrm{CN}$, and $U$-line \\
\hline 264 & $17,9-16_{7,9}$ & 216663.574 & 16 & 155 & 1.2 & 55 & Blend with $\mathrm{C}_{2} \mathrm{H}_{5} \mathrm{OH}, \mathrm{C}_{3} \mathrm{H}_{7} \mathrm{CN}$, and $U$-line \\
\hline 265 & $17_{8,10}-16_{7,10}$ & 216665.420 & 16 & 155 & 1.2 & 55 & Blend with $\mathrm{C}_{2} \mathrm{H}_{5} \mathrm{OH}, \mathrm{C}_{3} \mathrm{H}_{7} \mathrm{CN}$, and $U$-line \\
\hline 266 & $17_{8,9}-16_{7,10}$ & 216665.567 & 16 & 155 & 17.9 & 55 & Blend with $\mathrm{C}_{2} \mathrm{H}_{5} \mathrm{OH}, \mathrm{C}_{3} \mathrm{H}_{7} \mathrm{CN}$, and $U$-line \\
\hline 267 & $61_{4,57}-61_{4,58}{ }^{\star}$ & 216666.695 & 128 & 715 & 4.3 & 55 & Blend with $\mathrm{C}_{2} \mathrm{H}_{5} \mathrm{OH}, \mathrm{C}_{3} \mathrm{H}_{7} \mathrm{CN}$, and $U$-line \\
\hline 269 & $61_{5,57}-61_{4,58}{ }^{\star}$ & 216666.699 & 128 & 715 & 29.9 & 55 & Blend with $\mathrm{C}_{2} \mathrm{H}_{5} \mathrm{OH}, \mathrm{C}_{3} \mathrm{H}_{7} \mathrm{CN}$, and $U$-line \\
\hline 271 & $19_{7,13}-18_{6,12}$ & 217192.379 & 16 & 163 & 16.1 & 50 & Strong $\mathrm{CH}_{3} \mathrm{OCH}_{3}$ \\
\hline 272 & $30_{7,23}-29_{7,22}$ & 217285.278 & 10 & 258 & 118.8 & 50 & Blend with ${ }^{13} \mathrm{CN}, \mathrm{CH}_{2}{ }^{13} \mathrm{CHCN}$, and $U$-line \\
\hline 273 & $22_{6,17}-21_{5,16}$ & 217407.902 & 17 & 181 & 13.6 & 50 & Strong $\mathrm{HC}^{13} \mathrm{CCN},{ }^{13} \mathrm{CH}_{3} \mathrm{OH}, \mathrm{HCC}{ }^{13} \mathrm{CN}$, and ${ }^{13} \mathrm{CN}$ in absorption \\
\hline 274 & $21_{2,20}-20_{1,20}$ & 217409.248 & 52 & 160 & 0.3 & 50 & Strong $\mathrm{HC}^{13} \mathrm{CCN},{ }^{13} \mathrm{CH}_{3} \mathrm{OH}, \mathrm{HCC}{ }^{13} \mathrm{CN}$, and ${ }^{13} \mathrm{CN}$ in absorption \\
\hline 275 & $19_{7,12}-18_{6,13}$ & 217414.071 & 16 & 163 & 16.1 & 50 & Strong $\mathrm{HCC}^{13} \mathrm{CN}$ and ${ }^{13} \mathrm{CN}$ in absorption \\
\hline 276 & $31_{5,27}-30_{5,26}$ & 217441.193 & 100 & 260 & 125.2 & 50 & Strong ${ }^{13} \mathrm{CN}$ in absorption, $\mathrm{CH}_{2}{ }^{13} \mathrm{CHCN}$, and $\mathrm{CH}_{2} \mathrm{CH}^{13} \mathrm{CN}$ \\
\hline 277 & $31_{18,13}-30_{18,12}$ * & 219461.424 & 8 & 352 & 86.0 & 92 & Strong $\mathrm{C}_{2} \mathrm{H}_{5} \mathrm{CN}$ \\
\hline 279 & $31_{19,12}-30_{19,11}$ * & 219462.695 & 8 & 363 & 81.0 & 92 & Strong $\mathrm{C}_{2} \mathrm{H}_{5} \mathrm{CN}$ \\
\hline 281 & $31_{17,14}-30_{17,13}{ }^{\star}$ & 219476.189 & 8 & 341 & 90.7 & 92 & Strong $\mathrm{NH}_{2} \mathrm{CN}$ \\
\hline 283 & $31_{20,11}-30_{20,10}$ * & 219477.375 & 9 & 375 & 75.7 & 92 & Strong $\mathrm{NH}_{2} \mathrm{CN}$ \\
\hline 285 & $31_{21,10}-30_{21,9}{ }^{\star}$ & 219503.439 & 9 & 388 & 70.2 & 92 & Strong $\mathrm{C}_{2} \mathrm{H}_{5} \mathrm{CN}$ \\
\hline 287 & $31_{16,15}-30_{16,14}$ * & 219510.475 & 8 & 331 & 95.1 & 92 & Strong $\mathrm{C}_{2} \mathrm{H}_{5} \mathrm{CN}$ \\
\hline 289 & $31_{22,9}-30_{22,8^{\star}}$ & 219539.304 & 9 & 401 & 64.4 & 92 & Strong $\mathrm{HNCO}, v_{5}=1$ and $\mathrm{HNCO}$ \\
\hline 291 & $31_{15,16}-30_{15,15}$ * & 219569.004 & 8 & 321 & 99.3 & 92 & Strong $\mathrm{C}^{18} \mathrm{O}$ \\
\hline 293 & $62_{17,46}-61_{18,43}$ & 219569.366 & 161 & 836 & 17.0 & 92 & Strong $\mathrm{C}^{18} \mathrm{O}$ \\
\hline 294 & $62_{17,45}-61_{18,44}$ & 219572.725 & 161 & 836 & 17.0 & 92 & Strong $\mathrm{C}^{18} \mathrm{O}$ \\
\hline 295 & $31_{24,7}-30_{24,6}{ }^{\star}$ & 219635.627 & 11 & 429 & 52.0 & 92 & Blend with $\mathrm{C}_{2} \mathrm{H}_{5} \mathrm{CN}, v_{20}=1$ and $\mathrm{CH}_{3} \mathrm{CN}, v_{4}=1$ \\
\hline 297 & $32_{3,29}-31_{4,28}$ & 219637.655 & 7 & 266 & 43.4 & 92 & Blend with $\mathrm{C}_{2} \mathrm{H}_{5} \mathrm{CN}, v_{20}=1$ and $\mathrm{CH}_{3} \mathrm{CN}, v_{4}=1$ \\
\hline 298 & $31_{14,17}-30_{14,16}$ * & 219658.346 & 7 & 312 & 103.2 & 92 & Strong $\mathrm{NH}_{2} \mathrm{CN}$ and $\mathrm{HNCO}$ \\
\hline 300 & $32_{4,29}-31_{4,28}$ & 219781.349 & 100 & 266 & 130.1 & 92 & Strong $\mathrm{HNCO}, v_{6}=1$ and $\mathrm{HNCO}$ \\
\hline 301 & $31_{13,19}-30_{13,18}$ * & 219787.930 & 7 & 304 & 106.9 & 92 & Strong $\mathrm{HNCO}$ \\
\hline 303 & $21_{6,15}-20_{5,16}$ & 219793.028 & 100 & 173 & 13.1 & 92 & Strong HNCO \\
\hline 304 & $34_{1,34}-33_{0,33}{ }^{\star}$ & 220205.386 & 100 & 270 & 71.6 & 98 & Strong $\mathrm{HNCO}, v_{5}=1$ and $\mathrm{C}_{2} \mathrm{H}_{5} \mathrm{CN}, v_{13}=1 / v_{21}=1$ \\
\hline 306 & $34_{1,34}-33_{1,33}{ }^{\star}$ & 220205.400 & 17 & 270 & 141.6 & 98 & Strong $\mathrm{HNCO}, v_{5}=1$ and $\mathrm{C}_{2} \mathrm{H}_{5} \mathrm{CN}, v_{13}=1 / v_{21}=1$ \\
\hline 308 & $58_{18,41}-58_{17,41}$ * & 220209.432 & 15 & 764 & 2.8 & 98 & Strong $\mathrm{HNCO}, v_{5}=1$ and $\mathrm{C}_{2} \mathrm{H}_{5} \mathrm{CN}, v_{13}=1 / v_{21}=1$ \\
\hline 310 & $58_{18,41}-58_{17,42^{\star}}$ & 220209.852 & 15 & 764 & 68.8 & 98 & Strong $\mathrm{HNCO}, v_{5}=1$ and $\mathrm{C}_{2} \mathrm{H}_{5} \mathrm{CN}, v_{13}=1 / v_{21}=1$ \\
\hline 312 & $31_{11,21}-30_{11,20}$ & 220231.977 & 7 & 289 & 113.4 & 98 & Blend with $\mathrm{CH}_{3} \mathrm{CN}$ and $U$-line \\
\hline 313 & $31_{11,20}-30_{11,19}$ & 220232.321 & 7 & 289 & 113.4 & 98 & Blend with $\mathrm{CH}_{3} \mathrm{CN}$ and $U$-line \\
\hline 314 & $31_{6,26}-30_{6,25}$ & 220580.985 & 100 & 264 & 124.3 & 98 & Strong $\mathrm{CH}_{3}{ }^{13} \mathrm{CN}$ and $\mathrm{HNCO}$ \\
\hline 315 & $31_{10,22}-30_{10,21}$ & 220603.352 & 7 & 283 & 116.2 & 98 & Strong $\mathrm{CH}_{3} \mathrm{CN}, \mathrm{CH}_{3}{ }^{13} \mathrm{CN}$, and $\mathrm{C}_{2} \mathrm{H}_{5} \mathrm{OH}$ \\
\hline 316 & $62_{4,58}-62_{3,59}{ }^{\star}$ & 220606.847 & 154 & 735 & 30.0 & 98 & Strong $\mathrm{CH}_{3}{ }^{13} \mathrm{CN}$ and $\mathrm{C}_{2} \mathrm{H}_{5} \mathrm{OH}$ \\
\hline 318 & $62_{5,58}-62_{4,59}^{\star}$ & 220606.849 & 154 & 735 & 30.0 & 98 & Strong $\mathrm{CH}_{3}{ }^{13} \mathrm{CN}$ and $\mathrm{C}_{2} \mathrm{H}_{5} \mathrm{OH}$ \\
\hline 320 & $31_{10,21}-30_{10,20}$ & 220609.106 & 7 & 283 & 116.2 & 98 & Strong $\mathrm{CH}_{3}{ }^{13} \mathrm{CN}$ and $\mathrm{C}_{2} \mathrm{H}_{5} \mathrm{OH}$ \\
\hline 321 & $33_{3,30}-32_{4,29}$ & 226072.922 & 7 & 276 & 45.7 & 278 & Blend with c- $\mathrm{C}_{2} \mathrm{H}_{4} \mathrm{O}$ and ${ }^{13} \mathrm{CH}_{3} \mathrm{OH}$ \\
\hline 322 & $33_{4,30}-32_{4,29}$ & 226165.300 & 7 & 276 & 134.3 & 278 & Blend with $\mathrm{CN}$ in absorption and $\mathrm{CH}_{3} \mathrm{CH}_{3} \mathrm{CO}, v_{t}=1$ \\
\hline 323 & $33_{3,30}-32_{3,29}$ & 226216.618 & 100 & 276 & 134.3 & 278 & Blend with $\mathrm{CH}_{3} \mathrm{CH}_{3} \mathrm{CO}$ and $\mathrm{CN}$ in absorption \\
\hline 324 & $33_{4,30}-32_{3,29}$ & 226309.069 & 7 & 276 & 45.8 & 278 & Strong $\mathrm{CN}$ in absorption \\
\hline 325 & $32_{5,28}-31_{4,27}$ & 226357.720 & 9 & 270 & 34.1 & 278 & Strong $\mathrm{CN}$ in absorption \\
\hline 326 & $32,19,13-31_{19,12}^{\star}$ & 226534.021 & 8 & 373 & 86.7 & 278 & Blend with $\mathrm{CH}_{2} \mathrm{NH}$ \\
\hline 328 & $32_{18,15}-31_{18,14}$ ᄎ & 226537.002 & 100 & 362 & 91.5 & 278 & Blend with $\mathrm{CH}_{2} \mathrm{NH}$ \\
\hline 330 & $32_{20,13}-31_{20,12}$ * & 226545.568 & 8 & 385 & 81.6 & 278 & Blend with $\mathrm{CH}_{2} \mathrm{NH}$ \\
\hline 332 & $32_{17,15}-31_{17,14}$ * & 226557.411 & 8 & 351 & 96.1 & 278 & Blend with $\mathrm{C}_{2} \mathrm{H}_{5} \mathrm{CN}, v_{13}=1 / v_{21}=1$ \\
\hline 334 & $32_{21,11}-31_{21,10}$ * & 226569.424 & 9 & 398 & 76.2 & 278 & Blend with $\mathrm{CH}_{3} \mathrm{COOH}, \mathrm{C}_{2} \mathrm{H}_{5} \mathrm{OH}$, and $\mathrm{CN}$ in absorption \\
\hline 336 & $32_{16,16}-31_{16,15}$ * & 226599.078 & 8 & 341 & 100.4 & 278 & Blend with $\mathrm{C}_{2} \mathrm{H}_{5}{ }^{13} \mathrm{CN}$ \\
\hline 338 & $35_{0,35^{-}-34_{1,34}{ }^{\star}}$ & 226601.560 & 18 & 280 & 73.8 & 278 & Blend with $\mathrm{C}_{2} \mathrm{H}_{5}{ }^{13} \mathrm{CN}$ \\
\hline 340 & $35_{0,35}-34_{0,34}{ }^{\star}$ & 226601.561 & 18 & 280 & 145.8 & 278 & Blend with $\mathrm{C}_{2} \mathrm{H}_{5}{ }^{13} \mathrm{CN}$ \\
\hline 342 & $32_{22,10}-31_{22,9}{ }^{\star}$ & 226603.850 & 9 & 411 & 70.6 & 278 & Blend with $\mathrm{C}_{2} \mathrm{H}_{5}{ }^{13} \mathrm{CN}$ \\
\hline 344 & $32_{15,17}-31_{15,16}{ }^{\star}$ & 226667.222 & 8 & 332 & 104.5 & 96 & Strong $\mathrm{CN}$ in absorption and $\mathrm{C}_{2} \mathrm{H}_{5} \mathrm{OH}$ \\
\hline 346 & $32_{14,18}-31_{14,17}{ }^{\star}$ & 226769.121 & 8 & 323 & 108.3 & 96 & Strong $\mathrm{CN}$ in absorption \\
\hline 348 & $32_{13,20}-31_{13,19^{\star}}$ & 226915.261 & 8 & 314 & 111.8 & 96 & Strong $\mathrm{CN}$ in absorption \\
\hline 350 & $32_{12,21}-31_{12,20}$ * & 227121.372 & 7 & 307 & 115.1 & 96 & Blend with $\mathrm{C}_{2} \mathrm{H}_{5} \mathrm{CN}$, uncertain baseline \\
\hline
\end{tabular}


Table 2. continued.

\begin{tabular}{|c|c|c|c|c|c|c|c|}
\hline 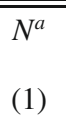 & $\begin{array}{c}\text { Transition }^{b} \\
\text { (2) }\end{array}$ & $\begin{array}{c}\text { Frequency } \\
(\mathrm{MHz}) \\
(3)\end{array}$ & $\begin{array}{c}\text { Unc. }^{c} \\
(\mathrm{kHz}) \\
(4)\end{array}$ & $\begin{array}{l}E_{1}{ }^{d} \\
(\mathrm{~K}) \\
(5)\end{array}$ & $\begin{array}{l}S \mu^{2} \\
\left(\mathrm{D}^{2}\right) \\
(6)\end{array}$ & $\begin{array}{c}\sigma^{e} \\
(\mathrm{mK}) \\
(7)\end{array}$ & $\begin{array}{c}\text { Comments } \\
\text { (8) }\end{array}$ \\
\hline 352 & $32_{11,22}-31_{11,21}$ & 227412.121 & 7 & 300 & 118.1 & 85 & Strong $\mathrm{HC}_{3} \mathrm{~N}$ \\
\hline 353 & $32_{11,21}-31_{11,20}$ & 227412.786 & 7 & 300 & 118.1 & 85 & Strong $\mathrm{HC}_{3} \mathrm{~N}$ \\
\hline 354 & $31_{6,25}-30_{6,24}$ & 227600.701 & 10 & 265 & 125.4 & 85 & Strong $\mathrm{C}_{2} \mathrm{H}_{5} \mathrm{OH}$ and $\mathrm{NH}_{2} \mathrm{CHO}$ \\
\hline 355 & $32_{10,23}-31_{10,22}$ & 227826.118 & 8 & 293 & 120.8 & 85 & Blend with $\mathrm{CH}_{3} \mathrm{OH}$ \\
\hline 356 & $32_{10,22}-31_{10,21}$ & 227836.414 & 8 & 293 & 120.8 & 85 & Blend with $\mathrm{CH}_{3} \mathrm{CH}_{3} \mathrm{CO}$ \\
\hline 357 & $22_{6,16}-21_{5,17}$ & 227855.315 & 20 & 180 & 12.7 & 85 & Blend with $\mathrm{HC}^{13} \mathrm{CCN}, v_{7}=2, \mathrm{HCC}^{13} \mathrm{CN}, v_{7}=2$, and $U$-line \\
\hline 358 & $81_{10,71}-81_{9,72}{ }^{\star}$ & 231205.772 & 1715 & 1244 & 77.8 & 183 & Strong ${ }^{13} \mathrm{CS}$ and $U$-line \\
\hline 360 & $34_{3,31}-33_{4,30}$ & 232486.365 & 7 & 287 & 48.0 & 19 & Strong $\mathrm{C}_{2} \mathrm{H}_{5}{ }^{13} \mathrm{CN}$ and $\mathrm{C}_{2} \mathrm{H}_{5} \mathrm{OH}$ \\
\hline 361 & $34_{4,31}-33_{4,30}$ & 232545.336 & 50 & 287 & 138.4 & 19 & Blend uith $\mathrm{C}_{2} \mathrm{H}_{5} \mathrm{CN}$ and $\mathrm{C}_{2} \mathrm{H}_{5} \mathrm{OH}$ \\
\hline 362 & $34_{3,31}-33_{3,30}$ & 232578.686 & 50 & 287 & 138.4 & 19 & Blend with $\mathrm{CH}_{3}{ }^{13} \mathrm{CH}_{2} \mathrm{CN}$ and ${ }^{13} \mathrm{CH}_{2} \mathrm{CHCN}$ \\
\hline 363 & $34_{4,31}-33_{3,30}$ & 232637.775 & 8 & 287 & 48.0 & 19 & Blend with $\mathrm{CH}_{3} \mathrm{OH}, v_{t}=1, \mathrm{CH}_{3} \mathrm{CH}_{3} \mathrm{CO}$, and ${ }^{13} \mathrm{CH}_{2} \mathrm{CO}$ \\
\hline 364 & $12_{12,0}-11_{11,1} \star$ & 232895.717 & 15 & 153 & 25.4 & 19 & Blend with $\mathrm{C}_{2} \mathrm{H}_{5} \mathrm{CN}$ and ${ }^{13} \mathrm{CH}_{3} \mathrm{CN}, v_{8}=1$ \\
\hline 366 & $12_{12,0}-11_{11,0}$ * & 232895.717 & 15 & 153 & 1.4 & 19 & Blend with $\mathrm{C}_{2} \mathrm{H}_{5} \mathrm{CN}$ and ${ }^{13} \mathrm{CH}_{3} \mathrm{CN}, v_{8}=1$ \\
\hline 368 & $33_{10,24-32_{10,23}}$ & 235059.537 & 50 & 304 & 125.4 & 131 & Strong $\mathrm{CH}_{3} \mathrm{OCHO}$ and $\mathrm{C}_{2} \mathrm{H}_{5} \mathrm{CN}$ \\
\hline 369 & $33_{10,23}-32_{10,22}$ & 235077.408 & 8 & 304 & 125.4 & 131 & Strong $\mathrm{CH}_{3} \mathrm{CH}_{3} \mathrm{CO}$ and $\mathrm{C}_{2} \mathrm{H}_{5} \mathrm{OH}$ \\
\hline 370 & $16_{10,7}-15_{9,6}{ }^{\star}$ & 235511.949 & 15 & 159 & 21.6 & 131 & Strong $\mathrm{HC}^{13} \mathrm{CCN}$ \\
\hline 372 & $16_{10,6}-15_{9,7}{ }^{\star}$ & 235511.949 & 15 & 159 & 21.6 & 131 & Strong $\mathrm{HC}^{13} \mathrm{CCN}$ \\
\hline 374 & $33_{9,25}-32_{9,24}$ & 235683.207 & 50 & 299 & 127.8 & 131 & Blend with $\mathrm{HCC}^{13} \mathrm{CN}, v_{5}=1 / v_{7}=3$ \\
\hline 375 & $33_{9,24}-32_{9,23}$ & 235875.700 & 8 & 299 & 127.8 & 131 & Strong ${ }^{13} \mathrm{CH}_{3} \mathrm{OH}$ and $\mathrm{CH}_{3} \mathrm{OCHO}$ \\
\hline 376 & $34_{4,30}-33_{5,29}$ & 235952.121 & 100 & 292 & 39.0 & 131 & Strong $\mathrm{C}_{2} \mathrm{H}_{5}{ }^{13} \mathrm{CN},{ }^{13} \mathrm{CH}_{3} \mathrm{OH}$, and SiS \\
\hline 377 & $33_{7,27}-32_{7,26}$ & 236011.028 & 50 & 289 & 131.6 & 131 & Strong ${ }^{13} \mathrm{CH}_{3} \mathrm{OH}$ \\
\hline 378 & $66_{4,62}-66_{3,63}{ }^{\star}$ & 236269.468 & 357 & 816 & 30.1 & 37 & Blend with $\mathrm{NH}_{2} \mathrm{CH}_{2} \mathrm{CN}$ and $\mathrm{H}_{2} \mathrm{C}^{34} \mathrm{~S}$ \\
\hline 380 & $66_{5,62}-66_{4,63}^{\star}$ & 236269.469 & 357 & 816 & 30.1 & 37 & Blend with $\mathrm{NH}_{2} \mathrm{CH}_{2} \mathrm{CN}$ and $\mathrm{H}_{2} \mathrm{C}^{34} \mathrm{~S}$ \\
\hline 382 & $33_{8,26}-32_{8,25}$ & 236274.518 & 50 & 294 & 130.0 & 37 & Blend with $\mathrm{NH}_{2} \mathrm{CH}_{2} \mathrm{CN}$ and $\mathrm{H}_{2} \mathrm{C}^{34} \mathrm{~S}$ \\
\hline 383 & $23_{6,17}-22_{5,18}$ & 236483.115 & 100 & 188 & 12.0 & 37 & Blend with $\mathrm{NH}_{2} \mathrm{CH}_{2} \mathrm{CN}$ and $\mathrm{CH}_{2}{ }^{13} \mathrm{CHCN}$ \\
\hline 384 & $34_{23,11}-33_{23,10}{ }^{\star}$ & 240768.473 & 9 & 447 & 77.2 & 216 & Strong $\mathrm{CH}_{3}{ }^{13} \mathrm{CH}_{2} \mathrm{CN}, \mathrm{CH}_{3} \mathrm{CH}_{3} \mathrm{CO}$, and $\mathrm{C}_{2} \mathrm{H}_{5} \mathrm{OH}$ \\
\hline 386 & $34_{16,18}-33_{16,17}^{\star}$ & 240777.277 & 8 & 363 & 110.8 & 216 & Strong $\mathrm{CH}_{3}{ }^{13} \mathrm{CH}_{2} \mathrm{CN}, \mathrm{CH}_{3} \mathrm{CH}_{3} \mathrm{CO}$, and $\mathrm{C}_{2} \mathrm{H}_{5} \mathrm{OH}$ \\
\hline 388 & $34_{15,19}-33_{15,18}{ }^{\star}$ & 240866.895 & 8 & 354 & 114.6 & 216 & Strong $\mathrm{C}_{2} \mathrm{H}_{5} \mathrm{CN}$ and $\mathrm{HNCO}$ \\
\hline 390 & $34_{14,21}-33_{14,20}{ }^{\star}$ & 240996.801 & 8 & 345 & 118.2 & 216 & Strong $\mathrm{C}_{2} \mathrm{H}_{5}{ }^{13} \mathrm{CN}, \mathrm{CH}_{3} \mathrm{CH}_{3} \mathrm{CO}$, and $\mathrm{C}^{34} \mathrm{~S}$ \\
\hline 392 & $33_{7,26}-32_{7,25}$ & 241104.938 & 12 & 290 & 132.3 & 216 & Blend with $\mathrm{CH}_{3}{ }^{13} \mathrm{CH}_{2} \mathrm{CN}$ and $U$-line \\
\hline 393 & $36_{2,34}-35_{3,33}$ & 241154.165 & 10 & 304 & 60.8 & 216 & Strong $\mathrm{CH}_{3} \mathrm{OH}, v_{t}=1$ \\
\hline 394 & $36_{3,34}-35_{3,33}$ & 241155.214 & 10 & 304 & 147.7 & 216 & Strong $\mathrm{CH}_{3} \mathrm{OH}, v_{t}=1$ \\
\hline 395 & $36_{2,34}-35_{2,33}$ & 241155.889 & 10 & 304 & 147.7 & 216 & Strong $\mathrm{CH}_{3} \mathrm{OH}, v_{t}=1$ \\
\hline 396 & $36_{3,34}-35_{2,33}$ & 241156.938 & 10 & 304 & 60.8 & 216 & Strong $\mathrm{CH}_{3} \mathrm{OH}, v_{t}=1$ \\
\hline 397 & $34_{29,5}-33_{29,4}$ * & 241179.811 & 17 & 543 & 38.8 & 216 & Strong $\mathrm{CH}_{3} \mathrm{OH}, v_{t}=1$ \\
\hline 399 & $34_{13,22}-33_{13,21}{ }^{\star}$ & 241179.912 & 8 & 337 & 121.5 & 216 & Strong $\mathrm{CH}_{3} \mathrm{OH}, v_{t}=1$ \\
\hline 401 & $65_{3,62}-65_{2,63^{\star}}$ & 241187.055 & 355 & 784 & 22.6 & 216 & Strong $\mathrm{CH}_{3} \mathrm{OH}, v_{t}=1$ \\
\hline 403 & $65_{3,62}-65_{3,63}^{\star}$ & 241187.055 & 355 & 784 & 3.4 & 216 & Strong $\mathrm{CH}_{3} \mathrm{OH}, v_{t}=1$ \\
\hline 405 & $21_{8,14}-20_{7,13}$ & 244391.993 & 15 & 180 & 18.5 & 46 & Strong ${ }^{13} \mathrm{CH}_{3} \mathrm{CH}_{2} \mathrm{CN}$ and $\mathrm{C}_{2} \mathrm{H}_{5} \mathrm{CN}$ \\
\hline 406 & $21_{8,13}-20_{7,13}$ & 244396.589 & 15 & 180 & 1.4 & 46 & Strong ${ }^{13} \mathrm{CH}_{3} \mathrm{CH}_{2} \mathrm{CN}$ and $\mathrm{C}_{2} \mathrm{H}_{5} \mathrm{CN}$ \\
\hline 407 & $23_{5,18}-22_{4,19}$ & 244399.583 & 45 & 185 & 5.5 & 46 & Strong ${ }^{13} \mathrm{CH}_{3} \mathrm{CH}_{2} \mathrm{CN}$ and $\mathrm{C}_{2} \mathrm{H}_{5} \mathrm{CN}$ \\
\hline 408 & $21_{8,14}-20_{7,14}$ & 244437.241 & 15 & 180 & 1.4 & 46 & Blend with $\mathrm{C}_{2} \mathrm{H}_{5} \mathrm{CN}, \mathrm{C}_{2} \mathrm{H}_{5} \mathrm{OH}$, and $U$-line \\
\hline 409 & $21_{8,13}-20_{7,14}$ & 244441.837 & 15 & 180 & 18.5 & 46 & Blend with $\mathrm{C}_{2} \mathrm{H}_{5} \mathrm{CN}, \mathrm{C}_{2} \mathrm{H}_{5} \mathrm{OH}$, and $U$-line \\
\hline 410 & $23_{7,16}-22_{6,17}$ & 245129.808 & 16 & 191 & 16.1 & 72 & Strong $\mathrm{CH}_{2} \mathrm{NH}$ \\
\hline 411 & $36_{3,33}-35_{4,32}$ & 245273.854 & 100 & 310 & 52.6 & 72 & Strong $\mathrm{HC}_{3} \mathrm{~N}, v_{4}=1$ and $\mathrm{C}_{2} \mathrm{H}_{5}{ }^{13} \mathrm{CN}$ \\
\hline 412 & $36_{4,33}-35_{4,32}$ & 245297.601 & 100 & 310 & 146.7 & 72 & Blend with $\mathrm{CH}_{3} \mathrm{CH}_{3} \mathrm{CO}$ and ${ }^{34} \mathrm{SO}_{2}$ \\
\hline 413 & $36_{3,33}-35_{3,32}$ & 245311.367 & 100 & 310 & 146.7 & 72 & Blend with $\mathrm{C}_{2} \mathrm{H}_{5} \mathrm{OH}$ and $\mathrm{SO}_{2}$ \\
\hline 414 & $36_{4,33}-35_{3,32}$ & 245335.158 & 100 & 310 & 52.6 & 72 & Strong $\mathrm{SO}_{2}$ \\
\hline 415 & $34_{8,26}-33_{8,25}$ & 245447.840 & 11 & 305 & 134.4 & 72 & Strong $\mathrm{C}_{2} \mathrm{H}_{5} \mathrm{OH}, \mathrm{C}_{2} \mathrm{H}_{5} \mathrm{CN}, v_{13}=1 / v_{21}=1$, and ${ }^{13} \mathrm{CH}_{3} \mathrm{CH}_{2} \mathrm{CN}$ \\
\hline 416 & $38_{0,38}-37_{0,37}^{\star}$ & 245779.482 & 100 & 314 & 158.3 & 53 & Strong $\mathrm{CH}_{2}{ }^{13} \mathrm{CHCN}$ and $\mathrm{C}_{2} \mathrm{H}_{5} \mathrm{CN}, v_{13}=1 / v_{21}=1$ \\
\hline 418 & $38_{0,38}-37_{1,37^{\star}}$ & 245779.550 & 18 & 314 & 80.5 & 53 & Strong $\mathrm{CH}_{2}{ }^{13} \mathrm{CHCN}$ and $\mathrm{C}_{2} \mathrm{H}_{5} \mathrm{CN}, v_{13}=1 / v_{21}=1$ \\
\hline 420 & $24_{6,18}-23_{5,19}$ & 245842.016 & 26 & 196 & 11.2 & 53 & Blend with $\mathrm{C}_{2} \mathrm{H}_{3} \mathrm{CN}, v_{11}=1 / v_{15}=1$ \\
\hline 421 & $14_{12,2}-13_{11,3^{\star}}$ & 247059.416 & 14 & 162 & 25.4 & 68 & Strong $\mathrm{CH}_{3} \mathrm{OCHO}$ \\
\hline 423 & $14_{12,2}-13_{11,2}^{\star}$ & 247059.416 & 14 & 162 & 1.5 & 68 & Strong $\mathrm{CH}_{3} \mathrm{OCHO}$ \\
\hline 425 & $35_{6,30}-34_{6,29}$ & 247073.069 & 100 & 308 & 141.1 & 68 & Strong $\mathrm{C}_{2} \mathrm{H}_{3} \mathrm{CN}$ and $\mathrm{CH}_{2}{ }^{13} \mathrm{CHCN}$ \\
\hline 426 & $39_{0,39}-38_{0,38^{\star}}$ & 252168.489 & 100 & 326 & 162.5 & 42 & Blend with $\mathrm{C}_{2} \mathrm{H}_{5} \mathrm{CN}, v_{13}=1 / v_{21}=1$ \\
\hline 428 & $39_{0,39}-38_{1,38}{ }^{\star}$ & 252168.541 & 18 & 326 & 82.7 & 42 & Blend with $\mathrm{C}_{2} \mathrm{H}_{5} \mathrm{CN}, v_{13}=1 / v_{21}=1$ \\
\hline 430 & $24_{7,17}-23_{6,18}$ & 252254.714 & 16 & 199 & 16.0 & 42 & Strong $\mathrm{CH}_{3} \mathrm{OH}$ \\
\hline 431 & $25_{7,19}-24_{6,18}$ & 254026.124 & 15 & 207 & 16.1 & 32 & $\begin{array}{l}\text { Strong } \mathrm{CH}_{3} \mathrm{OH} \text { in absorption, } \mathrm{C}_{2} \mathrm{H}_{5} \mathrm{CN}, v_{13}=1 / v_{21}=1 \text {, } \\
\text { and } \mathrm{HC}^{13} \mathrm{CCN}, v_{6}=1\end{array}$ \\
\hline 432 & $15_{12,3}-14_{11,4}^{\star}$ & 254139.046 & 14 & 167 & 25.5 & 32 & Strong $\mathrm{C}_{2} \mathrm{H}_{3} \mathrm{CN}$ \\
\hline 434 & $15_{12,3}-14_{11,3^{\star}}$ & 254139.046 & 14 & 167 & 1.5 & 32 & Strong $\mathrm{C}_{2} \mathrm{H}_{3} \mathrm{CN}$ \\
\hline
\end{tabular}


A. Belloche et al.: Detection and chemical modeling of ethyl formate and $n$-propyl cyanide in Sgr B2(N), Online Material $p 14$

Table 2. continued.

\begin{tabular}{|c|c|c|c|c|c|c|c|}
\hline$N^{a}$ & Transition $^{b}$ & $\begin{array}{c}\text { Frequency } \\
\text { (MHz) } \\
\text { (3) }\end{array}$ & $\begin{array}{l}\text { Unc. }^{c} \\
(\mathrm{kHz}) \\
(4)\end{array}$ & $\begin{array}{l}E_{1}^{d} \\
(\mathrm{~K}) \\
(5)\end{array}$ & $\begin{array}{l}S \mu^{2} \\
\left(\mathrm{D}^{2}\right) \\
(6)\end{array}$ & $\begin{array}{c}\sigma^{e} \\
(\mathrm{mK}) \\
(7)\end{array}$ & $\begin{array}{c}\text { Comments } \\
\text { (8) }\end{array}$ \\
\hline 436 & $36_{14,23}-35_{14,22}{ }^{\star}$ & 255232.834 & 8 & 368 & 127.8 & 217 & Strong $\mathrm{CH}_{3} \mathrm{OH}$ \\
\hline 438 & $17_{11,6}-16_{10,7^{\star}}$ & 255442.928 & 14 & 171 & 23.8 & 217 & Strong $\mathrm{CH}_{2} \mathrm{CH}^{13} \mathrm{CN}$ \\
\hline 440 & $17_{11,6}-16_{10,6}{ }^{\star}$ & 255442.928 & 14 & 171 & 1.4 & 217 & Strong $\mathrm{CH}_{2} \mathrm{CH}^{13} \mathrm{CN}$ \\
\hline 442 & $36_{13,24}-35_{13,23}{ }^{\star}$ & 255458.533 & 8 & 360 & 131.0 & 217 & Strong $\mathrm{C}_{2} \mathrm{H}_{5} \mathrm{CN}, v_{13}=1 / v_{21}=1$ and $\mathrm{CH}_{2}{ }^{13} \mathrm{CHCN}$ \\
\hline 444 & $37_{4,33}-36_{5,32}$ & 255646.981 & 8 & 327 & 46.2 & 217 & Strong $\mathrm{H}^{13} \mathrm{CCCN}$ and $\mathrm{NH}_{2} \mathrm{CHO}, v_{12}=1$ \\
\hline 445 & $23_{8,16}-22_{7,15}$ & 257978.249 & 15 & 195 & 18.7 & 1127 & Strong $\mathrm{CH}_{3} \mathrm{CN}, v_{8}=1$ \\
\hline 446 & $56_{11,46}-55_{12,43}$ & 258029.375 & 88 & 664 & 12.8 & 1127 & Strong $\mathrm{CH}_{3} \mathrm{CN}, v_{8}=1$ \\
\hline 447 & $38_{3,35}-37_{4,34}$ & 258032.591 & 9 & 334 & 57.1 & 1127 & Strong $\mathrm{CH}_{3} \mathrm{CN}, v_{8}=1$ \\
\hline 448 & $38_{4,35}-37_{4,34}$ & 258042.009 & 9 & 334 & 155.1 & 1127 & Strong $\mathrm{CH}_{3} \mathrm{CN}, v_{8}=1$ \\
\hline 449 & $38_{3,35}-37_{3,34}$ & 258047.578 & 9 & 334 & 155.1 & 1127 & Strong $\mathrm{CH}_{3} \mathrm{CN}, v_{8}=1$ \\
\hline 450 & $38_{4,35}-37_{3,34}$ & 258056.996 & 9 & 334 & 57.1 & 1127 & Strong $\mathrm{CH}_{3} \mathrm{CN}, v_{8}=1$ \\
\hline 451 & $23_{8,15}-22_{7,16}$ & 258162.637 & 15 & 195 & 18.7 & 1127 & Strong ${ }^{13} \mathrm{CH}_{3} \mathrm{OH}$ and $\mathrm{HC}^{15} \mathrm{~N}$ \\
\hline 452 & $36_{9,27}-35_{9,26}$ & 258237.747 & 10 & 334 & 141.2 & 1127 & Strong $\mathrm{CH}_{3} \mathrm{CN}, v_{8}=1$ and $\mathrm{SO}$ \\
\hline 453 & $36_{6,31}-35_{5,30}$ & 258252.184 & 13 & 320 & 33.8 & 1127 & Strong $\mathrm{CH}_{3} \mathrm{CN}, v_{8}=1$ and $\mathrm{SO}$ \\
\hline 454 & $26_{7,20}-25_{6,19}$ & 258801.772 & 16 & 216 & 15.9 & 1609 & Strong $\mathrm{CH}_{3}{ }^{13} \mathrm{CH}_{2} \mathrm{CN}$ \\
\hline 455 & $37_{6,32}-36_{6,31}$ & 260009.847 & 8 & 332 & 149.4 & 413 & Strong $\mathrm{C}_{2} \mathrm{H}_{5} \mathrm{OH}, \mathrm{CH}_{2}{ }^{13} \mathrm{CHCN}$, and $\mathrm{CH}_{3} \mathrm{OCH}_{3}$ \\
\hline 456 & $39_{2,37}-38_{3,36}$ & 260297.586 & 10 & 340 & 67.4 & 413 & Strong $\mathrm{C}_{2} \mathrm{H}_{5} \mathrm{CN}, v_{13}=1 / v_{21}=1$ \\
\hline 457 & $39_{3,37}-38_{3,36}$ & 260297.819 & 10 & 340 & 160.2 & 413 & Strong $\mathrm{C}_{2} \mathrm{H}_{5} \mathrm{CN}, v_{13}=1 / v_{21}=1$ \\
\hline 458 & $39_{2,37}-38_{2,36}$ & 260297.972 & 10 & 340 & 160.2 & 413 & Strong $\mathrm{C}_{2} \mathrm{H}_{5} \mathrm{CN}, v_{13}=1 / v_{21}=1$ \\
\hline 459 & $39_{3,37}-38_{2,36}$ & 260298.205 & 10 & 340 & 67.4 & 413 & Strong $\mathrm{C}_{2} \mathrm{H}_{5} \mathrm{CN}, v_{13}=1 / v_{21}=1$ \\
\hline 460 & $37_{7,31}-36_{7,30}$ & 263345.617 & 9 & 337 & 148.6 & 74 & Blend with $\mathrm{HCC}^{13} \mathrm{CN}, v_{7}=1$ \\
\hline 461 & $37_{6,32}-36_{5,31}$ & 263354.897 & 11 & 332 & 36.3 & 74 & Blend with $\mathrm{NH}_{2} \mathrm{CH}_{2} \mathrm{CN}$ \\
\hline 462 & $37_{11,27}-36_{11,26}$ & 263432.848 & 8 & 358 & 141.1 & 74 & Strong $\mathrm{HNCO}, v_{5}=1$ and $\mathrm{HNCO}$ \\
\hline 463 & $37_{11,26}-36_{11,25}$ & 263445.456 & 8 & 358 & 141.2 & 74 & Strong $\mathrm{HNCO}, v_{5}=1$ and $\mathrm{HNCO}$ \\
\hline 464 & $20_{10,11}-19_{9,10} \star$ & 263669.118 & 14 & 183 & 22.4 & 108 & Strong $\mathrm{CH}_{3} \mathrm{OCH}_{3}, \mathrm{HNCO}$, and $\mathrm{HC}^{13} \mathrm{CCN}, v_{7}=1$ \\
\hline 466 & $20_{10,11}-19_{9,11}^{\star}$ & 263669.145 & 14 & 183 & 1.4 & 108 & Strong $\mathrm{CH}_{3} \mathrm{OCH}_{3}, \mathrm{HNCO}$, and $\mathrm{HC}^{13} \mathrm{CCN}, v_{7}=1$ \\
\hline 468 & $36_{7,29}-35_{7,28}$ & 264087.850 & 11 & 326 & 145.6 & 108 & Blend with $\mathrm{H}_{2}{ }^{13} \mathrm{CS}$ and $U$-line \\
\hline 469 & $37_{10,28}-36_{10,27}$ & 264094.028 & 9 & 351 & 143.5 & 108 & Blend with $\mathrm{H}_{2}{ }^{13} \mathrm{CS}$ and $U$-line \\
\hline 470 & $40_{2,38}-39_{3,37}$ & 266674.808 & 10 & 352 & 69.7 & 91 & Blend with $\mathrm{O}^{13} \mathrm{CS}$ and $\mathrm{C}_{2} \mathrm{H}_{5}{ }^{13} \mathrm{CN}$ \\
\hline 471 & $40_{3,38}-39_{3,37}^{\star}$ & 266674.948 & 10 & 352 & 164.4 & 91 & Blend with $\mathrm{O}^{13} \mathrm{CS}$ and $\mathrm{C}_{2} \mathrm{H}_{5}{ }^{13} \mathrm{CN}$ \\
\hline 473 & $40_{3,38}-39_{2,37}$ & 266675.181 & 10 & 352 & 69.7 & 91 & Blend with $\mathrm{O}^{13} \mathrm{CS}$ and $\mathrm{C}_{2} \mathrm{H}_{5}{ }^{13} \mathrm{CN}$ \\
\hline 474 & $15_{13,2}-14_{12,3^{\star}}$ & 266949.479 & 14 & 174 & 27.6 & 91 & Blend with $\mathrm{SO}_{2}$ and $U$-line \\
\hline 476 & 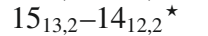 & 266949.479 & 14 & 174 & 1.6 & 91 & Blend with $\mathrm{SO}_{2}$ and $U$-line \\
\hline 478 & $38_{5,33}-37_{5,32}$ & 267143.852 & 100 & 344 & 153.6 & 91 & Strong $\mathrm{NH}_{2}{ }^{13} \mathrm{CHO}, \mathrm{CH}_{3} \mathrm{CH}_{3} \mathrm{CO}$, and $\mathrm{C}_{2} \mathrm{H}_{5} \mathrm{CN}, v_{13}=1 / v_{21}=1$ \\
\hline
\end{tabular}

Notes: ${ }^{a}$ Numbering of the observed transitions associated with a modeled line stronger than $20 \mathrm{mK} .{ }^{b}$ Transitions marked with a ${ }^{\star}$ are double with a frequency difference less than $0.1 \mathrm{MHz}$. The quantum numbers of the second one are not shown. ${ }^{c}$ Frequency uncertainty. ${ }^{d}$ Lower energy level in temperature units $\left(E_{\mathrm{l}} / k_{\mathrm{B}}\right){ }^{e}$ Calculated rms noise level in $T_{\mathrm{mb}}$ scale. 
Table 6. Transitions of anti-n-propyl cyanide, employed in the present fits, their frequencies $(\mathrm{MHz})$, uncertainties Unc. $(\mathrm{kHz})$, and residuals $\mathrm{O}-\mathrm{C}(\mathrm{kHz})$ between frequencies measured in the laboratory and those calculated from the final spectroscopic parameters. Unresolved asymmetry splitting (two transitions having the same $K_{a}$ and the same transition frequency) has been treated as intensity-weighted average of the two lines.

\begin{tabular}{|c|c|c|c|c|c|}
\hline$\overline{J^{\prime}, K_{a}^{\prime}, K_{c}^{\prime}}$ & - & $\overline{J^{\prime \prime}, K_{a}^{\prime \prime}, K_{c}^{\prime \prime}}$ & Frequency & Unc. & $\overline{\overline{\mathrm{O}-\mathrm{C}}}$ \\
\hline $2,1,2$ & - & $1,1,1$ & 8727.068 & 10 & -3 \\
\hline $2,0,2$ & - & $1,0,1$ & 8841.749 & 10 & 4 \\
\hline $2,1,1$ & - & $1,1,0$ & 8957.437 & 10 & 3 \\
\hline $4,1,4$ & - & $3,1,3$ & 17453.162 & 10 & 12 \\
\hline $3,0,3$ & - & $2,0,2$ & 13261.436 & 5 & 1 \\
\hline $3,1,3$ & - & $2,1,2$ & 13090.304 & 5 & 8 \\
\hline $3,1,2$ & - & $2,1,1$ & 13435.836 & 5 & 3 \\
\hline $3,2,2$ & - & $2,2,1$ & 13263.547 & 5 & -2 \\
\hline $3,2,1$ & - & $2,2,0$ & 13265.404 & 5 & -0 \\
\hline $4,2,3$ & - & $3,2,2$ & 17684.317 & 5 & -10 \\
\hline $4,3,2$ & - & $3,3,1$ & 17686.050 & 5 & 2 \\
\hline $4,3,1$ & - & $3,3,0$ & 17686.050 & 5 & 2 \\
\hline $4,2,2$ & - & $3,2,1$ & 17688.965 & 5 & 2 \\
\hline $5,3,3$ & - & $4,3,2$ & 22107.924 & 5 & 1 \\
\hline $5,3,2$ & - & $4,3,1$ & 22107.924 & 5 & 1 \\
\hline $5,4,2$ & - & $4,4,1$ & 22108.119 & 5 & 1 \\
\hline $5,4,1$ & - & $4,4,0$ & 22108.119 & 5 & 1 \\
\hline $9,1,8$ & - & $9,0,9$ & 24166.242 & 5 & 7 \\
\hline $10,1,9$ & - & $10,0,10$ & 24799.350 & 5 & 3 \\
\hline $11,1,10$ & - & $11,0,11$ & 25508.602 & 5 & 0 \\
\hline $12,1,11$ & - & $12,0,12$ & 26297.469 & 5 & -2 \\
\hline $11,2,10$ & - & $12,1,11$ & 6897.938 & 5 & -4 \\
\hline $16,1,15$ & - & $15,2,14$ & 13992.090 & 5 & -1 \\
\hline $17,1,16$ & - & $16,2,15$ & 19339.460 & 5 & 1 \\
\hline $19,1,19$ & - & $18,2,16$ & 6197.075 & 5 & -1 \\
\hline $17,3,15$ & - & $18,2,16$ & 26095.072 & 5 & -3 \\
\hline $18,3,16$ & - & $19,2,17$ & 21310.053 & 5 & 1 \\
\hline $19,3,16$ & - & $20,2,19$ & 19831.330 & 5 & 5 \\
\hline $20,3,18$ & - & $21,2,19$ & 11569.979 & 5 & -1 \\
\hline $21,3,19$ & - & $22,2,20$ & 6607.331 & 5 & -0 \\
\hline $22,3,19$ & - & $23,2,22$ & 7318.534 & 5 & 2 \\
\hline $26,2,25$ & - & $25,3,22$ & 4813.248 & 5 & -1 \\
\hline $26,2,24$ & - & $25,3,23$ & 13932.516 & 5 & -7 \\
\hline $28,2,26$ & - & $27,3,25$ & 24644.757 & 5 & -0 \\
\hline $28,2,27$ & - & $27,3,24$ & 12629.848 & 5 & 4 \\
\hline $29,2,28$ & - & $28,3,25$ & 16441.572 & 5 & -1 \\
\hline $30,2,29$ & - & $29,3,26$ & 20181.553 & 5 & -0 \\
\hline $31,2,30$ & - & $30,3,27$ & 23843.940 & 5 & 0 \\
\hline $27,4,24$ & - & $28,3,25$ & 25160.533 & 5 & 3 \\
\hline $27,4,23$ & - & $28,3,26$ & 26014.363 & 5 & -8 \\
\hline $28,4,25$ & - & $29,3,26$ & 20523.876 & 5 & 5 \\
\hline $28,4,24$ & - & $29,3,27$ & 21572.582 & 5 & -10 \\
\hline $29,4,26$ & - & $30,3,27$ & 15856.700 & 5 & 6 \\
\hline $29,4,25$ & - & $30,3,28$ & 17135.390 & 5 & -4 \\
\hline $30,4,27$ & - & $31,3,28$ & 11155.783 & 5 & 2 \\
\hline $30,4,26$ & - & $31,3,29$ & 12704.192 & 5 & -2 \\
\hline $31,4,28$ & - & $32,3,29$ & 6417.726 & 5 & 3 \\
\hline $31,4,27$ & - & $32,3,30$ & 8280.581 & 5 & 2 \\
\hline $36,3,33$ & - & $35,4,32$ & 12979.624 & 5 & 4 \\
\hline $36,3,34$ & - & $35,4,31$ & 9299.790 & 5 & -2 \\
\hline $37,3,35$ & - & $36,4,32$ & 13655.067 & 5 & -4 \\
\hline $38,3,35$ & - & $37,4,34$ & 22999.972 & 5 & 6 \\
\hline $39,3,37$ & - & $38,4,34$ & 22299.091 & 5 & 3 \\
\hline $37,5,32$ & - & $38,4,35$ & 24129.041 & 5 & 4 \\
\hline $38,5,34$ & - & $39,4,35$ & 19274.638 & 5 & -2 \\
\hline $38,5,33$ & - & $39,4,36$ & 19621.858 & 5 & 1 \\
\hline $39,5,34$ & - & $40,4,37$ & 15110.833 & 5 & 0 \\
\hline $35,0,35$ & - & $34,0,34$ & 152420.07 & 50 & 10 \\
\hline $35,2,33$ & - & $34,2,32$ & 156362.10 & 50 & -19 \\
\hline $35,6,29$ & - & $34,6,28$ & 154794.60 & 50 & 5 \\
\hline
\end{tabular}

Table 6. continued.

\begin{tabular}{|c|c|c|c|c|c|}
\hline$\overline{J^{\prime}, K_{a}^{\prime}, K_{c}^{\prime}}$ & - & $\overline{\overline{J^{\prime \prime}}, K_{a}^{\prime \prime}, K_{c}^{\prime \prime}}$ & Frequency & Unc. & $\overline{\mathrm{O}-\mathrm{C}}$ \\
\hline $35,6,30$ & - & $34,6,29$ & 154794.60 & 50 & 5 \\
\hline $35,7,28$ & - & $34,7,27$ & 154778.85 & 50 & -24 \\
\hline $35,7,29$ & - & $34,7,28$ & 154778.85 & 50 & -24 \\
\hline $35,8,27$ & - & $34,8,26$ & 154773.84 & 50 & -40 \\
\hline $35,8,28$ & - & $34,8,27$ & 154773.84 & 50 & -40 \\
\hline $35,9,26$ & - & $34,9,25$ & 154775.62 & 50 & -38 \\
\hline $35,9,27$ & - & $34,9,26$ & 154775.62 & 50 & -38 \\
\hline $35,10,26$ & - & $34,10,25$ & 154782.18 & 50 & -3 \\
\hline $35,10,25$ & - & $34,10,24$ & 154782.18 & 50 & -3 \\
\hline $35,11,25$ & - & $34,11,24$ & 154792.35 & 50 & 22 \\
\hline $35,11,24$ & - & $34,11,23$ & 154792.35 & 50 & 22 \\
\hline $35,12,24$ & - & $34,12,23$ & 154805.40 & 50 & -28 \\
\hline $35,12,23$ & - & $34,12,22$ & 154805.40 & 50 & -28 \\
\hline $36,0,36$ & - & $35,0,35$ & 156709.22 & 50 & -7 \\
\hline $36,1,36$ & - & $35,1,35$ & 156376.78 & 50 & -9 \\
\hline $36,2,34$ & - & $35,2,33$ & 160851.40 & 50 & -55 \\
\hline $36,2,35$ & - & $35,2,34$ & 158511.09 & 50 & 15 \\
\hline $36,3,33$ & - & $35,3,32$ & 159816.09 & 50 & -55 \\
\hline $36,3,34$ & - & $35,3,33$ & 159295.56 & 50 & -7 \\
\hline $36,4,32$ & - & $35,4,31$ & 159360.35 & 50 & -6 \\
\hline $36,4,33$ & - & $35,4,32$ & 159324.49 & 50 & -4 \\
\hline $36,5,31$ & - & $35,5,30$ & 159258.14 & 50 & -38 \\
\hline $36,5,32$ & - & $35,5,31$ & 159256.97 & 50 & 14 \\
\hline $36,6,31$ & - & $35,6,30$ & 159218.77 & 50 & -44 \\
\hline $36,6,30$ & - & $35,6,29$ & 159218.77 & 50 & -44 \\
\hline $36,7,30$ & - & $35,7,29$ & 159201.12 & 50 & 4 \\
\hline $36,7,29$ & - & $35,7,28$ & 159201.12 & 50 & 4 \\
\hline $36,8,29$ & - & $35,8,28$ & 159194.99 & 50 & -15 \\
\hline $36,8,28$ & - & $35,8,27$ & 159194.99 & 50 & -15 \\
\hline $36,13,24$ & - & $35,13,23$ & 159241.59 & 50 & 7 \\
\hline $36,13,23$ & - & $35,13,22$ & 159241.59 & 50 & 7 \\
\hline $36,14,23$ & - & $35,14,22$ & 159259.85 & 50 & 6 \\
\hline $36,14,22$ & - & $35,14,21$ & 159259.85 & 50 & 6 \\
\hline $36,15,22$ & - & $35,15,21$ & 159280.32 & 50 & 26 \\
\hline $36,15,21$ & - & $35,15,20$ & 159280.32 & 50 & 26 \\
\hline $36,16,21$ & - & $35,16,20$ & 159302.83 & 50 & 28 \\
\hline $36,16,20$ & - & $35,16,19$ & 159302.83 & 50 & 28 \\
\hline $36,17,20$ & - & $35,17,19$ & 159327.32 & 50 & 42 \\
\hline $36,17,19$ & - & $35,17,18$ & 159327.32 & 50 & 42 \\
\hline $36,18,19$ & - & $35,18,18$ & 159353.76 & 50 & 100 \\
\hline $36,18,18$ & - & $35,18,17$ & 159353.76 & 50 & 100 \\
\hline $37,0,37$ & - & $36,0,36$ & 160998.16 & 50 & 12 \\
\hline $37,1,37$ & - & $36,1,36$ & 160691.64 & 50 & 25 \\
\hline $48,1,47$ & - & $47,1,46$ & 211856.19 & 50 & 11 \\
\hline $48,2,47$ & - & $47,2,46$ & 210741.48 & 50 & -115 \\
\hline $48,2,46$ & - & $47,2,45$ & 214343.68 & 50 & 15 \\
\hline $48,3,46$ & - & $47,3,45$ & 212219.54 & 50 & 25 \\
\hline $48,4,45$ & - & $47,4,44$ & 212517.79 & 50 & 5 \\
\hline $48,4,44$ & - & $47,4,43$ & 212766.64 & 50 & -12 \\
\hline $48,5,44$ & - & $47,5,43$ & 212415.06 & 50 & -32 \\
\hline $48,5,43$ & - & $47,5,42$ & 212430.89 & 50 & 12 \\
\hline $48,6,43$ & - & $47,6,42$ & 212320.28 & 50 & 40 \\
\hline $48,6,42$ & - & $47,6,41$ & 212320.77 & 50 & -59 \\
\hline $48,7,42$ & - & $47,7,41$ & 212267.79 & 50 & -29 \\
\hline $48,7,41$ & - & $47,7,40$ & 212267.79 & 50 & -29 \\
\hline $48,8,41$ & - & $47,8,40$ & 212241.20 & 50 & 2 \\
\hline $48,8,40$ & - & $47,8,39$ & 212241.20 & 50 & 2 \\
\hline $48,9,40$ & - & $47,9,39$ & 212230.19 & 50 & -42 \\
\hline $48,9,39$ & - & $47,9,38$ & 212230.19 & 50 & -42 \\
\hline $48,10,39$ & - & $47,10,38$ & 212229.67 & 50 & 28 \\
\hline $48,10,38$ & - & $47,10,37$ & 212229.67 & 50 & 28 \\
\hline $48,12,37$ & - & $47,12,36$ & 212249.06 & 50 & -46 \\
\hline $48,12,36$ & - & $47,12,35$ & 212249.06 & 50 & -46 \\
\hline $48,13,36$ & - & $47,13,35$ & 212266.32 & 50 & -39 \\
\hline $48,13,35$ & - & $47,13,34$ & 212266.32 & 50 & -39 \\
\hline $48,14,35$ & - & $47,14,34$ & 212287.59 & 50 & 14 \\
\hline
\end{tabular}


A. Belloche et al.: Detection and chemical modeling of ethyl formate and $n$-propyl cyanide in Sgr B2(N), Online Material $p 16$

Table 6. continued.

\begin{tabular}{|c|c|c|c|c|c|}
\hline$\overline{J^{\prime}, K_{a}^{\prime}, K_{c}^{\prime}}$ & $\overline{-}$ & 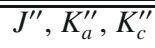 & Frequency & Unc. & $\overline{\mathrm{O}-\mathrm{C}}$ \\
\hline $48,14,34$ & - & $47,14,33$ & 212287.59 & 50 & 14 \\
\hline $48,15,34$ & - & $47,15,33$ & 212312.29 & 50 & -1 \\
\hline $48,15,33$ & - & $47,15,32$ & 212312.29 & 50 & -1 \\
\hline $48,16,33$ & - & $47,16,32$ & 212340.19 & 50 & 3 \\
\hline $48,16,32$ & - & $47,16,31$ & 212340.19 & 50 & 3 \\
\hline $48,17,32$ & - & $47,17,31$ & 212371.06 & 50 & 18 \\
\hline $48,17,31$ & - & $47,17,30$ & 212371.06 & 50 & 18 \\
\hline $48,18,31$ & - & $47,18,30$ & 212404.72 & 50 & 21 \\
\hline $48,18,30$ & - & $47,18,29$ & 212404.72 & 50 & 21 \\
\hline $48,19,30$ & - & $47,19,29$ & 212441.11 & 50 & 64 \\
\hline $48,19,29$ & - & $47,19,28$ & 212441.11 & 50 & 64 \\
\hline $48,20,29$ & - & $47,20,28$ & 212480.03 & 50 & 26 \\
\hline $48,20,28$ & - & $47,20,27$ & 212480.03 & 50 & 26 \\
\hline $48,21,28$ & - & $47,21,27$ & 212521.49 & 50 & -24 \\
\hline $48,21,27$ & - & $47,21,26$ & 212521.49 & 50 & -24 \\
\hline $48,22,27$ & - & $47,22,26$ & 212565.54 & 50 & 2 \\
\hline $48,22,26$ & - & $47,22,25$ & 212565.54 & 50 & 2 \\
\hline $48,23,26$ & - & $47,23,25$ & 212611.99 & 50 & -58 \\
\hline $48,23,25$ & - & $47,23,24$ & 212611.99 & 50 & -58 \\
\hline $49,0,49$ & - & $48,0,48$ & 212476.62 & 50 & 100 \\
\hline $49,1,49$ & - & $48,1,48$ & 212378.48 & 50 & 62 \\
\hline $49,2,48$ & - & $48,2,47$ & 215077.17 & 50 & 14 \\
\hline $49,4,46$ & - & $48,4,45$ & 216949.09 & 50 & -14 \\
\hline $49,16,34$ & - & $48,16,33$ & 216757.71 & 50 & 9 \\
\hline $49,16,33$ & - & $48,16,32$ & 216757.71 & 50 & 9 \\
\hline $49,17,33$ & - & $48,17,32$ & 216789.07 & 50 & 45 \\
\hline $49,17,32$ & - & $48,17,31$ & 216789.07 & 50 & 45 \\
\hline $49,20,30$ & - & $48,20,29$ & 216899.87 & 50 & 3 \\
\hline $49,20,29$ & - & $48,20,28$ & 216899.87 & 50 & 3 \\
\hline $50,0,50$ & - & $49,0,49$ & 216767.70 & 50 & -21 \\
\hline $50,3,48$ & - & $49,3,47$ & 221009.11 & 50 & 7 \\
\hline $50,7,44$ & - & $49,7,43$ & 221112.20 & 50 & -26 \\
\hline $50,7,43$ & - & $49,7,42$ & 221112.20 & 50 & -26 \\
\hline $50,8,43$ & - & $49,8,42$ & 221080.73 & 50 & -13 \\
\hline $50,8,42$ & - & $49,8,41$ & 221080.73 & 50 & -13 \\
\hline $50,9,42$ & - & $49,9,41$ & 221066.76 & 50 & -28 \\
\hline $50,9,41$ & - & $49,9,40$ & 221066.76 & 50 & -28 \\
\hline $50,10,41$ & - & $49,10,40$ & 221064.35 & 50 & -25 \\
\hline $50,10,40$ & - & $49,10,39$ & 221064.35 & 50 & -25 \\
\hline $50,11,40$ & - & $49,11,39$ & 221070.19 & 50 & -11 \\
\hline $50,11,39$ & - & $49,11,38$ & 221070.19 & 50 & -11 \\
\hline $50,12,39$ & - & $49,12,38$ & 221082.34 & 50 & 28 \\
\hline $50,12,38$ & - & $49,12,37$ & 221082.34 & 50 & 28 \\
\hline $50,13,38$ & - & $49,13,37$ & 221099.49 & 50 & -5 \\
\hline $50,13,37$ & - & $49,13,36$ & 221099.49 & 50 & -5 \\
\hline $50,14,37$ & - & $49,14,36$ & 221121.05 & 50 & 85 \\
\hline $50,14,36$ & - & $49,14,35$ & 221121.05 & 50 & 85 \\
\hline $50,20,31$ & - & $49,20,30$ & 221319.17 & 50 & -141 \\
\hline $50,20,30$ & - & $49,20,29$ & 221319.17 & 50 & -141 \\
\hline $51,0,51$ & - & $50,0,50$ & 221059.01 & 50 & -5 \\
\hline $51,1,51$ & - & $50,1,50$ & 220979.61 & 50 & 32 \\
\hline $64,6,59$ & - & $63,6,58$ & 283158.51 & 50 & -21 \\
\hline $64,6,58$ & - & $63,6,57$ & 283171.68 & 50 & 33 \\
\hline $64,7,58$ & - & $63,7,57$ & 283022.98 & 50 & 14 \\
\hline $64,7,57$ & - & $63,7,56$ & 283022.98 & 50 & 14 \\
\hline $64,8,57$ & - & $63,8,56$ & 282943.15 & 50 & 24 \\
\hline $64,8,56$ & - & $63,8,55$ & 282943.15 & 50 & 24 \\
\hline $64,9,56$ & - & $63,9,55$ & 282898.81 & 50 & 1 \\
\hline $64,9,55$ & - & $63,9,54$ & 282898.81 & 50 & 1 \\
\hline $64,10,55$ & - & $63,10,54$ & 282877.00 & 50 & -26 \\
\hline $64,10,54$ & - & $63,10,53$ & 282877.00 & 50 & -26 \\
\hline $64,11,54$ & - & $63,11,53$ & 282870.71 & 50 & -11 \\
\hline $64,11,53$ & - & $63,11,52$ & 282870.71 & 50 & -11 \\
\hline $64,12,53$ & - & $63,12,52$ & 282875.72 & 50 & -41 \\
\hline $64,12,52$ & - & $63,12,51$ & 282875.72 & 50 & -41 \\
\hline
\end{tabular}

Table 6. continued.

\begin{tabular}{rcccrr}
\hline \hline$J^{\prime}, K_{a}^{\prime}, K_{c}^{\prime}$ & - & $J^{\prime \prime}, K_{a}^{\prime \prime}, K_{c}^{\prime \prime}$ & Frequency & Unc. & O-C \\
\hline $64,13,52$ & - & $63,13,51$ & 282889.55 & 50 & -38 \\
$64,13,51$ & - & $63,13,50$ & 282889.55 & 50 & -38 \\
$64,14,51$ & - & $63,14,50$ & 282910.51 & 50 & -38 \\
$64,14,50$ & - & $63,14,49$ & 282910.51 & 50 & -38 \\
$64,15,50$ & - & $63,15,49$ & 282937.54 & 50 & 6 \\
$64,15,49$ & - & $63,15,48$ & 282937.54 & 50 & 6 \\
$64,16,49$ & - & $63,16,48$ & 282969.81 & 50 & 25 \\
$64,16,48$ & - & $63,16,47$ & 282969.81 & 50 & 25 \\
$64,17,48$ & - & $63,17,47$ & 283006.76 & 50 & -3 \\
$64,17,47$ & - & $63,17,46$ & 283006.76 & 50 & -3 \\
$64,18,47$ & - & $63,18,46$ & 283048.15 & 50 & 64 \\
$64,18,46$ & - & $63,18,45$ & 283048.15 & 50 & 64 \\
$64,19,46$ & - & $63,19,45$ & 283093.46 & 50 & -14 \\
$64,19,45$ & - & $63,19,44$ & 283093.46 & 50 & -14 \\
$64,20,45$ & - & $63,20,44$ & 283142.77 & 50 & 47 \\
$64,20,44$ & - & $63,20,43$ & 283142.77 & 50 & 47 \\
$64,21,44$ & - & $63,21,43$ & 283195.66 & 50 & -22 \\
$64,21,43$ & - & $63,21,42$ & 283195.66 & 50 & -22 \\
\hline
\end{tabular}


Table 7. Transitions of gauche- $n$-propyl cyanide, employed in the present fits, their frequencies $(\mathrm{MHz})$, uncertainties Unc. $(\mathrm{kHz})$, and residuals $\mathrm{O}-\mathrm{C}(\mathrm{kHz})$ between frequencies measured in the laboratory and those calculated from the final spectroscopic parameters. Unresolved asymmetry splitting (two transitions having the same $K_{a}$ and the same transition frequency) has been treated as intensityweighted average of the two lines.

\begin{tabular}{|c|c|c|c|c|c|}
\hline$\overline{J^{\prime}, K_{a}^{\prime}, K_{c}^{\prime}}$ & - & 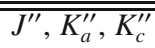 & Frequency & "Unc. & $\overline{\mathrm{O}-\mathrm{C}}$ \\
\hline $2,0,2$ & - & $1,0,1$ & 11912.654 & 10 & -18 \\
\hline $2,1,2$ & - & $1,1,1$ & 11384.045 & 10 & -0 \\
\hline $2,1,1$ & - & $1,1,0$ & 12508.374 & 10 & -10 \\
\hline $3,0,3$ & - & $2,0,2$ & 17785.949 & 10 & 6 \\
\hline $3,1,3$ & - & $2,1,2$ & 17055.610 & 5 & 1 \\
\hline $2,1,2$ & - & $1,0,1$ & 18176.781 & 5 & 1 \\
\hline $4,1,3$ & - & $4,0,4$ & 10286.601 & 5 & 0 \\
\hline $5,1,4$ & - & $5,0,5$ & 12167.868 & 5 & -3 \\
\hline $6,1,5$ & - & $6,0,6$ & 14609.404 & 5 & 1 \\
\hline $7,1,6$ & - & $7,0,7$ & 17634.822 & 5 & 1 \\
\hline $2,2,1$ & - & $2,1,2$ & 22064.261 & 5 & 0 \\
\hline $24,3,21$ & - & $25,2,24$ & 5073.952 & 5 & 0 \\
\hline $10,3,7$ & - & $9,4,6$ & 12670.827 & 5 & -2 \\
\hline $11,3,9$ & - & $10,4,6$ & 16546.819 & 5 & -3 \\
\hline $28,3,26$ & - & $27,4,23$ & 19891.929 & 5 & 1 \\
\hline $36,4,32$ & - & $37,3,35$ & 19547.899 & 5 & 1 \\
\hline $13,4,10$ & - & $12,5,7$ & 15362.409 & 5 & 0 \\
\hline $38,4,35$ & - & $37,5,32$ & 9991.617 & 5 & 0 \\
\hline $39,4,36$ & - & $38,5,33$ & 5311.217 & 5 & 1 \\
\hline $15,5,10$ & - & $14,6,9$ & 13597.769 & 5 & 1 \\
\hline $12,7,6$ & - & $13,6,7$ & 13549.701 & 5 & -1 \\
\hline $12,7,5$ & - & $13,6,8$ & 13551.095 & 5 & 5 \\
\hline $13,7,7$ & - & $14,6,8$ & 7398.401 & 5 & -2 \\
\hline $13,7,6$ & - & $14,6,9$ & 7401.855 & 5 & 4 \\
\hline $15,8,8$ & - & $16,7,9$ & 9498.238 & 5 & -4 \\
\hline $15,8,7$ & - & $16,7,10$ & 9498.794 & 5 & -5 \\
\hline $19,7,12$ & - & $18,8,11$ & 9123.186 & 5 & -1 \\
\hline $19,7,13$ & - & $18,8,10$ & 9116.781 & 5 & -1 \\
\hline $20,7,14$ & - & $19,8,11$ & 15398.422 & 5 & 0 \\
\hline $22,8,14$ & - & $21,9,13$ & 13221.090 & 5 & -0 \\
\hline $22,8,15$ & - & $21,9,12$ & 13218.792 & 5 & -2 \\
\hline $23,8,15$ & - & $22,9,14$ & 19511.211 & 5 & 2 \\
\hline $23,8,16$ & - & $22,9,13$ & 19506.528 & 5 & 0 \\
\hline $19,10,10$ & - & $20,9,11$ & 13681.725 & 5 & -1 \\
\hline $19,10,9$ & - & $20,9,12$ & 13681.725 & 5 & -1 \\
\hline $25,9,17$ & - & $24,10,14$ & 17310.401 & 5 & -6 \\
\hline $25,9,16$ & - & $24,10,15$ & 17311.214 & 5 & 2 \\
\hline $26,9,18$ & - & $25,10,15$ & 23599.302 & 5 & 2 \\
\hline $26,9,17$ & - & $25,10,16$ & 23600.945 & 5 & 11 \\
\hline $28,10,19$ & - & $27,11,16$ & 21388.390 & 5 & -9 \\
\hline $28,10,18$ & - & $27,11,17$ & 21388.670 & 5 & -6 \\
\hline $26,13,14$ & - & $27,12,15$ & 13813.369 & 5 & -3 \\
\hline $26,13,13$ & - & $27,12,16$ & 13813.369 & 5 & -3 \\
\hline $22,4,18$ & - & $22,4,19$ & 25543.715 & 5 & -1 \\
\hline $25,5,20$ & - & $25,5,21$ & 16254.957 & 5 & 2 \\
\hline $26,5,21$ & - & $26,5,22$ & 21090.090 & 5 & -1 \\
\hline $30,6,24$ & - & $30,6,25$ & 16246.388 & 5 & 1 \\
\hline $31,6,25$ & - & $31,6,26$ & 21145.902 & 5 & 1 \\
\hline $34,7,27$ & - & $34,7,28$ & 11727.780 & 5 & -2 \\
\hline $36,7,29$ & - & $36,7,30$ & 20572.880 & 5 & -2 \\
\hline $37,7,30$ & - & $37,7,31$ & 26237.933 & 5 & 4 \\
\hline $39,8,31$ & - & $39,8,32$ & 11013.037 & 5 & -2 \\
\hline $25,5,21$ & - & $24,4,20$ & 182990.23 & 50 & 19 \\
\hline $26,6,21$ & - & $25,6,20$ & 157628.25 & 50 & 16 \\
\hline $26,7,19$ & - & $25,7,18$ & 157477.90 & 50 & 8 \\
\hline $26,10,17$ & - & $25,10,16$ & 156296.53 & 50 & -59 \\
\hline $26,10,16$ & - & $25,10,15$ & 156296.53 & 50 & -59 \\
\hline $26,11,16$ & - & $25,11,15$ & 156141.79 & 50 & 65 \\
\hline $26,11,15$ & - & $25,11,14$ & 156141.79 & 50 & 65 \\
\hline $26,12,15$ & - & $25,12,14$ & 156032.20 & 50 & -22 \\
\hline
\end{tabular}

Table 7. continued.

\begin{tabular}{|c|c|c|c|c|c|}
\hline$\overline{J^{\prime}, K_{a}^{\prime}, K_{c}^{\prime}}$ & - & $\overline{J^{\prime \prime}, K_{a}^{\prime \prime}, K_{c}^{\prime \prime}}$ & Frequency & Unc. & $\overline{\mathrm{O}-\mathrm{C}}$ \\
\hline $26,12,14$ & - & $25,12,13$ & 156032.20 & 50 & -22 \\
\hline $26,13,14$ & - & $25,13,13$ & 155954.49 & 50 & -56 \\
\hline $26,13,13$ & - & $25,13,12$ & 155954.49 & 50 & -56 \\
\hline $26,14,13$ & - & $25,14,12$ & 155900.13 & 50 & -0 \\
\hline $26,14,12$ & - & $25,14,11$ & 155900.13 & 50 & -0 \\
\hline $26,15,12$ & - & $25,15,11$ & 155863.29 & 50 & -10 \\
\hline $26,15,11$ & - & $25,15,10$ & 155863.29 & 50 & -10 \\
\hline $26,17,10$ & - & $25,17,9$ & 155827.91 & 50 & -33 \\
\hline $26,17,9$ & - & $25,17,8$ & 155827.91 & 50 & -33 \\
\hline $26,18,9$ & - & $25,18,8$ & 155824.64 & 50 & -20 \\
\hline $26,18,8$ & - & $25,18,7$ & 155824.64 & 50 & -20 \\
\hline $26,19,8$ & - & $25,19,7$ & 155828.81 & 50 & -17 \\
\hline $26,19,7$ & - & $25,19,6$ & 155828.81 & 50 & -17 \\
\hline $28,1,27$ & - & $27,1,26$ & 157495.25 & 50 & -33 \\
\hline $28,2,27$ & - & $27,1,26$ & 157503.23 & 50 & -51 \\
\hline $28,1,27$ & - & $27,2,26$ & 157482.64 & 50 & 46 \\
\hline $28,2,27$ & - & $27,2,26$ & 157490.60 & 50 & 8 \\
\hline $29,5,25$ & - & $28,4,24$ & 189805.28 & 50 & 48 \\
\hline $30,4,27$ & - & $29,3,26$ & 178167.69 & 50 & 31 \\
\hline $30,4,26$ & - & $29,4,25$ & 182703.13 & 50 & -23 \\
\hline $30,5,26$ & - & $29,4,25$ & 192210.43 & 50 & 32 \\
\hline $30,7,23$ & - & $29,7,22$ & 182961.27 & 50 & -29 \\
\hline $31,2,29$ & - & $30,2,28$ & 177803.90 & 50 & -13 \\
\hline $31,3,29$ & - & $30,2,28$ & 177857.94 & 50 & -1 \\
\hline $31,2,29$ & - & $30,3,28$ & 177721.22 & 50 & 6 \\
\hline $31,3,29$ & - & $30,3,28$ & 177775.23 & 50 & -12 \\
\hline $31,3,28$ & - & $30,3,27$ & 182248.22 & 50 & -22 \\
\hline $31,4,28$ & - & $30,3,27$ & 183077.55 & 50 & 26 \\
\hline $31,3,28$ & - & $30,4,27$ & 181050.65 & 50 & 36 \\
\hline $31,4,27$ & - & $30,5,26$ & 178318.43 & 50 & 156 \\
\hline $31,5,26$ & - & $30,5,25$ & 192803.61 & 50 & 6 \\
\hline $31,7,25$ & - & $30,7,24$ & 188156.51 & 50 & -20 \\
\hline $31,7,24$ & - & $30,7,23$ & 189515.12 & 50 & -74 \\
\hline $31,8,23$ & - & $30,8,22$ & 187858.13 & 50 & 9 \\
\hline $32,2,30$ & - & $31,2,29$ & 183183.36 & 50 & 18 \\
\hline $32,3,30$ & - & $31,2,29$ & 183218.50 & 50 & 28 \\
\hline $32,2,30$ & - & $31,3,29$ & 183129.35 & 50 & 37 \\
\hline $32,3,30$ & - & $31,3,29$ & 183164.45 & 50 & 6 \\
\hline $32,4,29$ & - & $31,3,28$ & 188119.54 & 50 & -5 \\
\hline $32,4,28$ & - & $31,4,27$ & 192927.96 & 50 & -13 \\
\hline $32,4,28$ & - & $31,5,27$ & 185714.09 & 50 & 6 \\
\hline $32,10,23$ & - & $31,10,22$ & 192852.63 & 50 & -4 \\
\hline $32,10,22$ & - & $31,10,21$ & 192854.42 & 50 & -4 \\
\hline $32,12,21$ & - & $31,12,20$ & 192320.23 & 50 & 5 \\
\hline $32,12,20$ & - & $31,12,19$ & 192320.23 & 50 & 5 \\
\hline $32,13,20$ & - & $31,13,19$ & 192158.76 & 50 & -19 \\
\hline $32,13,19$ & - & $31,13,18$ & 192158.76 & 50 & -19 \\
\hline $32,14,19$ & - & $31,14,18$ & 192040.62 & 50 & 14 \\
\hline $32,14,18$ & - & $31,14,17$ & 192040.62 & 50 & 14 \\
\hline $33,2,31$ & - & $32,2,30$ & 188564.34 & 50 & -33 \\
\hline $33,3,31$ & - & $32,2,30$ & 188587.13 & 50 & 13 \\
\hline $33,2,31$ & - & $32,3,30$ & 188529.24 & 50 & -2 \\
\hline $33,3,31$ & - & $32,3,30$ & 188551.94 & 50 & -47 \\
\hline $33,3,30$ & - & $32,3,29$ & 192871.17 & 50 & -3 \\
\hline $33,4,30$ & - & $32,3,29$ & 193259.00 & 50 & 18 \\
\hline $33,3,30$ & - & $32,4,29$ & 192301.88 & 50 & -16 \\
\hline $33,4,30$ & - & $32,4,29$ & 192689.70 & 50 & -5 \\
\hline $33,4,29$ & - & $32,5,28$ & 192663.51 & 50 & 12 \\
\hline $34,2,32$ & - & $33,2,31$ & 193946.21 & 50 & -59 \\
\hline $34,3,32$ & - & $33,2,31$ & 193960.95 & 50 & 14 \\
\hline $34,2,32$ & - & $33,3,31$ & 193923.55 & 50 & 26 \\
\hline $34,3,32$ & - & $33,3,31$ & 193938.16 & 50 & -32 \\
\hline $34,6,28$ & - & $33,6,27$ & 212120.06 & 50 & 3 \\
\hline $35,6,29$ & - & $34,6,28$ & 218287.17 & 50 & -26 \\
\hline $35,7,29$ & - & $34,7,28$ & 212483.75 & 50 & 16 \\
\hline $35,8,28$ & - & $34,8,27$ & 212458.29 & 50 & -13 \\
\hline
\end{tabular}


A. Belloche et al.: Detection and chemical modeling of ethyl formate and $n$-propyl cyanide in Sgr B2(N), Online Material $p 18$

Table 7. continued.

\begin{tabular}{|c|c|c|c|c|c|}
\hline$\overline{J^{\prime}, K_{a}^{\prime}, K_{c}^{\prime}}$ & 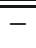 & $\overline{J^{\prime \prime}, K_{a}^{\prime \prime}, K_{c}^{\prime \prime}}$ & Frequency & Unc. & $\overline{\mathrm{O}-\mathrm{C}}$ \\
\hline $35,9,27$ & - & $34,9,26$ & 211828.06 & 50 & 142 \\
\hline $35,9,26$ & - & $34,9,25$ & 211938.01 & 50 & 7 \\
\hline $36,4,32$ & - & $35,5,31$ & 211527.01 & 50 & 14 \\
\hline $36,10,27$ & - & $35,10,26$ & 217402.38 & 50 & -15 \\
\hline $36,10,26$ & - & $35,10,25$ & 217418.12 & 50 & -2 \\
\hline $36,11,26$ & - & $35,11,25$ & 216940.59 & 50 & -30 \\
\hline $36,11,25$ & - & $35,11,24$ & 216941.74 & 50 & 33 \\
\hline $36,12,25$ & - & $35,12,24$ & 216609.17 & 50 & -33 \\
\hline $36,12,24$ & - & $35,12,23$ & 216609.17 & 50 & -33 \\
\hline $36,13,24$ & - & $35,13,23$ & 216367.23 & 50 & -1 \\
\hline $36,13,23$ & - & $35,13,22$ & 216367.23 & 50 & -1 \\
\hline $36,14,23$ & - & $35,14,22$ & 216188.17 & 50 & 23 \\
\hline $36,14,22$ & - & $35,14,21$ & 216188.17 & 50 & 23 \\
\hline $36,16,21$ & - & $35,16,20$ & 215956.57 & 50 & -26 \\
\hline $36,16,20$ & - & $35,16,19$ & 215956.57 & 50 & -26 \\
\hline $36,17,20$ & - & $35,17,19$ & 215885.18 & 50 & -12 \\
\hline $36,17,19$ & - & $35,17,18$ & 215885.18 & 50 & -12 \\
\hline $36,18,19$ & - & $35,18,18$ & 215835.30 & 50 & 9 \\
\hline $36,18,18$ & - & $35,18,17$ & 215835.30 & 50 & 9 \\
\hline $36,20,17$ & - & $35,20,16$ & 215784.95 & 50 & 23 \\
\hline $36,20,16$ & - & $35,20,15$ & 215784.95 & 50 & 23 \\
\hline $36,21,16$ & - & $35,21,15$ & 215779.22 & 50 & 45 \\
\hline $36,21,15$ & - & $35,21,14$ & 215779.22 & 50 & 45 \\
\hline $36,22,15$ & - & $35,22,14$ & 215783.85 & 50 & 5 \\
\hline $36,22,14$ & - & $35,22,13$ & 215783.85 & 50 & 5 \\
\hline $36,23,14$ & - & $35,23,13$ & 215797.53 & 50 & -19 \\
\hline $36,23,13$ & - & $35,23,12$ & 215797.53 & 50 & -19 \\
\hline $36,24,13$ & - & $35,24,12$ & 215819.22 & 50 & 36 \\
\hline $36,24,12$ & - & $35,24,11$ & 215819.22 & 50 & 36 \\
\hline $36,25,12$ & - & $35,25,11$ & 215847.81 & 50 & -52 \\
\hline $36,25,11$ & - & $35,25,10$ & 215847.81 & 50 & -52 \\
\hline $36,26,11$ & - & $35,26,10$ & 215882.85 & 50 & -12 \\
\hline $36,26,10$ & - & $35,26,9$ & 215882.85 & 50 & -12 \\
\hline $36,27,10$ & - & $35,27,9$ & 215923.60 & 50 & 10 \\
\hline $36,27,9$ & - & $35,27,8$ & 215923.60 & 50 & 10 \\
\hline $36,28,9$ & - & $35,28,8$ & 215969.54 & 50 & -15 \\
\hline $36,28,8$ & - & $35,28,7$ & 215969.54 & 50 & -15 \\
\hline $36,32,5$ & - & $35,32,4$ & 216198.45 & 50 & 4 \\
\hline $36,32,4$ & - & $35,32,3$ & 216198.45 & 50 & 4 \\
\hline $37,3,34$ & - & $36,3,33$ & 214265.30 & 50 & -8 \\
\hline $37,3,34$ & - & $36,4,33$ & 214147.29 & 50 & -26 \\
\hline $37,4,34$ & - & $36,4,33$ & 214225.81 & 50 & -13 \\
\hline $38,1,37$ & - & $37,2,36$ & 211401.39 & 50 & 59 \\
\hline $38,3,36$ & - & $37,2,35$ & 215476.23 & 50 & 36 \\
\hline $38,2,36$ & - & $37,3,35$ & 215469.85 & 50 & -35 \\
\hline $38,3,35$ & - & $37,3,34$ & 219628.21 & 50 & 19 \\
\hline $38,3,35$ & - & $37,4,34$ & 219549.67 & 50 & -13 \\
\hline $38,4,35$ & - & $37,4,34$ & 219601.64 & 50 & -40 \\
\hline $39,0,39$ & - & $38,1,38$ & 212743.02 & 50 & 6 \\
\hline $39,2,37$ & - & $38,2,36$ & 220854.26 & 50 & -8 \\
\hline $39,3,37$ & - & $38,2,36$ & 220855.81 & 50 & -18 \\
\hline $39,2,37$ & - & $38,3,36$ & 220851.79 & 50 & -24 \\
\hline $39,3,37$ & - & $38,3,36$ & 220853.34 & 50 & -33 \\
\hline $40,0,40$ & - & $39,1,39$ & 218129.30 & 50 & 18 \\
\hline $47,14,34$ & - & $46,14,33$ & 282874.18 & 50 & 42 \\
\hline $47,17,31$ & - & $46,17,30$ & 282070.21 & 50 & 4 \\
\hline $47,17,30$ & - & $46,17,29$ & 282070.21 & 50 & 4 \\
\hline $47,19,29$ & - & $46,19,28$ & 281801.94 & 50 & 20 \\
\hline $47,19,28$ & - & $46,19,27$ & 281801.94 & 50 & 20 \\
\hline $47,20,28$ & - & $46,20,27$ & 281716.83 & 50 & 33 \\
\hline $47,20,27$ & - & $46,20,26$ & 281716.83 & 50 & 33 \\
\hline $47,21,27$ & - & $46,21,26$ & 281656.67 & 50 & 10 \\
\hline $47,21,26$ & - & $46,21,25$ & 281656.67 & 50 & 10 \\
\hline $47,22,26$ & - & $46,22,25$ & 281617.41 & 50 & -44 \\
\hline
\end{tabular}

Table 7. continued.

\begin{tabular}{rlllrr}
\hline \hline$J^{\prime}, K_{a}^{\prime}, K_{c}^{\prime}$ & - & $J^{\prime \prime}, K_{a}^{\prime \prime}, K_{c}^{\prime \prime}$ & Frequency & Unc. & $\mathrm{O}-\mathrm{C}$ \\
\hline $47,22,25$ & - & $46,22,24$ & 281617.41 & 50 & -44 \\
$47,23,25$ & - & $46,23,24$ & 281596.02 & 50 & -16 \\
$47,23,24$ & - & $46,23,23$ & 281596.02 & 50 & -16 \\
$47,24,24$ & - & $46,24,23$ & 281589.94 & 50 & 8 \\
$47,24,23$ & - & $46,24,22$ & 281589.94 & 50 & 8 \\
$47,25,23$ & - & $46,25,22$ & 281597.15 & 50 & -18 \\
$47,25,22$ & - & $46,25,21$ & 281597.15 & 50 & -18 \\
$47,26,22$ & - & $46,26,21$ & 281616.14 & 50 & -10 \\
$47,26,21$ & - & $46,26,20$ & 281616.14 & 50 & -10 \\
$47,27,21$ & - & $46,27,20$ & 281645.57 & 50 & -9 \\
$47,27,20$ & - & $46,27,19$ & 281645.57 & 50 & -9 \\
$47,28,20$ & - & $46,28,19$ & 281684.43 & 50 & 48 \\
$47,28,19$ & - & $46,28,18$ & 281684.43 & 50 & 48 \\
$47,29,19$ & - & $46,29,18$ & 281731.64 & 50 & -30 \\
$47,29,18$ & - & $46,29,17$ & 281731.64 & 50 & -30 \\
$47,30,18$ & - & $46,30,17$ & 281786.66 & 50 & -36 \\
$47,30,17$ & - & $46,30,16$ & 281786.66 & 50 & -36 \\
$47,31,17$ & - & $46,31,16$ & 281848.86 & 50 & 31 \\
$47,31,16$ & - & $46,31,15$ & 281848.86 & 50 & 31 \\
$47,33,15$ & - & $46,33,14$ & 281992.38 & 50 & 34 \\
$47,33,14$ & - & $46,33,13$ & 281992.38 & 50 & 34 \\
$47,34,14$ & - & $46,34,13$ & 282072.85 & 50 & -22 \\
$47,34,13$ & - & $46,34,12$ & 282072.85 & 50 & -22 \\
$48,5,43$ & - & $47,5,42$ & 281724.06 & 50 & 0 \\
$48,6,43$ & - & $47,6,42$ & 281635.78 & 50 & -33 \\
\hline
\end{tabular}


A. Belloche et al.: Detection and chemical modeling of ethyl formate and $n$-propyl cyanide in Sgr B2(N), Online Material $p 19$

Table 9. Transitions of the anti-conformer of $n$-propyl cyanide observed with the IRAM $30 \mathrm{~m}$ telescope toward Sgr B2(N). The horizontal lines mark discontinuities in the observed frequency coverage. Only the transitions associated with a modeled line stronger than $20 \mathrm{mK}$ are listed.

\begin{tabular}{|c|c|c|c|c|c|c|c|}
\hline$N^{a}$ & Transition $^{b}$ & $\begin{array}{c}\text { Frequency } \\
\text { (MHz) } \\
\text { (3) }\end{array}$ & $\begin{array}{c}\text { Unc. }^{c} \\
(\mathrm{kHz}) \\
(4)\end{array}$ & $\begin{array}{l}E_{1}^{d} \\
(\mathrm{~K}) \\
(5)\end{array}$ & $\begin{array}{l}S \mu^{2} \\
\left(\mathrm{D}^{2}\right) \\
(6)\end{array}$ & $\begin{array}{c}\sigma^{e} \\
(\mathrm{mK}) \\
(7)\end{array}$ & $\begin{array}{l}\text { Comments } \\
\text { (8) }\end{array}$ \\
\hline 1 & $18_{1,17}-17_{1,16}$ & 80486.371 & 5 & 34 & 232.1 & 24 & Blend with $\mathrm{C}_{2} \mathrm{H}_{5} \mathrm{CN}, v_{13}=1 / v_{21}=1$ and $U$-line \\
\hline 2 & $19_{1,19}-18_{1,18}$ & 82778.264 & 4 & 37 & 245.1 & 17 & Blend with $\mathrm{HC}_{3} \mathrm{~N}, v_{5}=1 / v_{7}=3$ and $\mathrm{C}_{2} \mathrm{H}_{5} \mathrm{CN}$ \\
\hline 3 & $19_{0,19}-18_{0,18}$ & 83487.658 & 4 & 36 & 245.7 & 17 & Blend with $\mathrm{C}_{2} \mathrm{H}_{3} \mathrm{CN}$ and $U$-line \\
\hline 4 & $19_{2,18}-18_{2,17}$ & 83906.011 & 5 & 40 & 243.1 & 16 & Weak, blend with $U$-lines \\
\hline 5 & $19_{6,14}-18_{6,13}{ }^{\star}$ & 84021.555 & 4 & 73 & 221.3 & 19 & Group detected, partial blend with $\mathrm{C}_{2} \mathrm{H}_{5} \mathrm{CN}$ \\
\hline 7 & $19_{7,12}-18_{7,11}^{\star}$ & 84022.819 & 5 & 87 & 212.5 & 19 & Group detected, partial blend with $\mathrm{C}_{2} \mathrm{H}_{5} \mathrm{CN}$ \\
\hline 9 & $19_{5,15}-185,14^{\star}$ & 84023.956 & 4 & 62 & 228.8 & 19 & Group detected, partial blend with $\mathrm{C}_{2} \mathrm{H}_{5} \mathrm{CN}$ \\
\hline 11 & $19_{8,11}-18_{8,10}^{\star}$ & 84026.382 & 5 & 102 & 202.2 & 19 & Group detected, partial blend with $\mathrm{C}_{2} \mathrm{H}_{5} \mathrm{CN}$ \\
\hline 13 & $19_{9,10}-18_{9,9^{\star}}$ & 84031.619 & 5 & 120 & 190.6 & 19 & Blend with $\mathrm{C}_{2} \mathrm{H}_{5} \mathrm{CN}$ \\
\hline 15 & $19_{4,16}-18_{4,15}$ & 84033.299 & 4 & 53 & 234.9 & 19 & Blend with $\mathrm{C}_{2} \mathrm{H}_{5} \mathrm{CN}$ \\
\hline 16 & $19_{4,15}-18_{4,14}$ & 84033.712 & 4 & 53 & 234.9 & 19 & Blend with $\mathrm{C}_{2} \mathrm{H}_{5} \mathrm{CN}$ \\
\hline 17 & $19_{10,9}-18_{10,8^{\star}}$ & 84038.206 & 5 & 139 & 177.7 & 19 & Blend with $\mathrm{C}_{2} \mathrm{H}_{5} \mathrm{CN}$ \\
\hline 19 & $19_{11,8}-18_{11,7^{\star}}$ & 84045.967 & 5 & 161 & 163.4 & 19 & Blend with $U$-line \\
\hline 21 & $19_{3,17}-18_{3,16}$ & 84050.001 & 4 & 46 & 239.7 & 19 & Blend with $\mathrm{CH}_{3} \mathrm{CH}_{3} \mathrm{CO}$ \\
\hline 22 & $57_{3,54}-57_{2,55}{ }^{\star}$ & 84073.432 & 63 & 358 & 44.1 & 19 & Blend with $\mathrm{NH}_{2} \mathrm{CHO}$ \\
\hline 24 & $19_{14,5}-18_{14,4^{\star}}$ & 84075.429 & 7 & 238 & 112.4 & 19 & Blend with $\mathrm{NH}_{2} \mathrm{CHO}$ \\
\hline 26 & $19_{2,17}-18_{2,16}$ & 84408.080 & 4 & 40 & 243.1 & 19 & Blend with ${ }^{34} \mathrm{SO}$ \\
\hline 27 & $19_{1,18}-18_{1,17}$ & 84940.688 & 5 & 38 & 245.1 & 22 & Blend with $\mathrm{C}_{2} \mathrm{H}_{3} \mathrm{CN}$ \\
\hline 28 & $20_{1,20}-19_{1,19}$ & 87121.605 & 4 & 41 & 258.1 & 16 & Blend with $\mathrm{HN}^{13} \mathrm{C}$ in absorption \\
\hline 29 & $40_{1,39}-40_{1,40}$ & 87829.125 & 82 & 172 & 0.8 & 17 & Weak, partial blend with $\mathrm{C}_{2} \mathrm{H}_{5} \mathrm{OCHO}$ \\
\hline 30 & $20_{0,20}-19_{0,19}$ & 87829.384 & 5 & 40 & 258.6 & 17 & Weak, partial blend with $\mathrm{C}_{2} \mathrm{H}_{5} \mathrm{OCHO}$ \\
\hline 31 & $20_{2,19}-19_{2,18}$ & 88310.935 & 5 & 44 & 256.2 & 17 & Blend with $\mathrm{H}^{13} \mathrm{CCCN}, v_{6}=1$ and $\mathrm{CH}_{3} \mathrm{CH}_{3} \mathrm{CO}$ \\
\hline 32 & $20_{6,14}-19_{6,13^{\star}}$ & 88444.212 & 5 & 77 & 235.5 & 17 & $\begin{array}{l}\text { Group detected, partial blend with } \mathrm{CH}_{3} \mathrm{CH}_{3} \mathrm{CO}, \mathrm{C}_{2} \mathrm{H}_{5} \mathrm{OH} \text {, and } \\
U \text {-line? }\end{array}$ \\
\hline 34 & $20_{7,13}-19_{7,12}^{\star}$ & 88445.075 & 5 & 91 & 227.0 & 17 & $\begin{array}{l}\text { Group detected, partial blend with } \mathrm{CH}_{3} \mathrm{CH}_{3} \mathrm{CO}, \mathrm{C}_{2} \mathrm{H}_{5} \mathrm{OH} \text {, and } \\
U \text {-line? }\end{array}$ \\
\hline 36 & $20_{5,16}-19_{5,15^{\star}}$ & 88447.526 & 5 & 66 & 242.6 & 17 & $\begin{array}{l}\text { Group detected, partial blend with } \mathrm{CH}_{3} \mathrm{CH}_{3} \mathrm{CO}, \mathrm{C}_{2} \mathrm{H}_{5} \mathrm{OH} \text {, and } \\
U \text {-line? }\end{array}$ \\
\hline 38 & $20_{8,12}-19_{8,11}$ * & 88448.528 & 5 & 106 & 217.4 & 17 & $\begin{array}{l}\text { Group detected, partial blend with } \mathrm{CH}_{3} \mathrm{CH}_{3} \mathrm{CO}, \mathrm{C}_{2} \mathrm{H}_{5} \mathrm{OH} \text {, and } \\
U \text {-line? }\end{array}$ \\
\hline 40 & 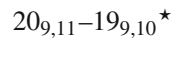 & 88453.836 & 5 & 124 & 206.4 & 17 & $\begin{array}{l}\text { Group detected, partial blend with } \mathrm{C}_{2} \mathrm{H}_{5}{ }^{13} \mathrm{CN},{ }^{13} \mathrm{CH}_{3} \mathrm{CH}_{2} \mathrm{CN} \text {, } \\
\text { and } \mathrm{CH}_{2}(\mathrm{OH}) \mathrm{CHO}\end{array}$ \\
\hline 42 & $20_{4,17}-19_{4,16}$ & 88458.794 & 5 & 57 & 248.4 & 17 & $\begin{array}{l}\text { Group detected, partial blend with } \mathrm{C}_{2} \mathrm{H}_{5}{ }^{13} \mathrm{CN},{ }^{13} \mathrm{CH}_{3} \mathrm{CH}_{2} \mathrm{CN} \text {, } \\
\text { and } \mathrm{CH}_{2}(\mathrm{OH}) \mathrm{CHO}\end{array}$ \\
\hline 43 & $20_{4,16}-19_{4,15}$ & 88459.387 & 5 & 57 & 248.4 & 17 & $\begin{array}{l}\text { Group detected, partial blend with } \mathrm{C}_{2} \mathrm{H}_{5}{ }^{13} \mathrm{CN},{ }^{13} \mathrm{CH}_{3} \mathrm{CH}_{2} \mathrm{CN} \text {, } \\
\text { and } \mathrm{CH}_{2}(\mathrm{OH}) \mathrm{CHO}\end{array}$ \\
\hline 44 & $20_{10,10}-19_{10,9^{\star}}$ & 88460.625 & 5 & 143 & 194.1 & 17 & $\begin{array}{l}\text { Group detected, partial blend with } \mathrm{C}_{2} \mathrm{H}_{5}{ }^{13} \mathrm{CN},{ }^{13} \mathrm{CH}_{3} \mathrm{CH}_{2} \mathrm{CN} \text {, } \\
\text { and } \mathrm{CH}_{2}(\mathrm{OH}) \mathrm{CHO}\end{array}$ \\
\hline 46 & $20_{11,9}-19_{11,8^{\star}}$ & 88468.687 & 5 & 165 & 180.5 & 17 & Weak \\
\hline 48 & $20_{3,18}-19_{3,17}$ & 88477.246 & 5 & 50 & 252.9 & 17 & Blend with $\mathrm{HNCO}, v_{4}=1$ and $\mathrm{C}_{2} \mathrm{H}_{5} \mathrm{CN}$ \\
\hline 49 & $20_{12,8}-19_{12,7^{\star}}$ & 88477.898 & 6 & 188 & 165.6 & 17 & Blend with $\mathrm{HNCO}, v_{4}=1$ and $\mathrm{C}_{2} \mathrm{H}_{5} \mathrm{CN}$ \\
\hline 51 & $20_{3,17}-19_{3,16}$ & 88507.586 & 5 & 50 & 252.9 & 17 & Blend with $\mathrm{C}_{2} \mathrm{H}_{5} \mathrm{CN}, v_{13}=1 / v_{21}=1$ and $\mathrm{H}^{13} \mathrm{CCCN}, v_{7}=1$ \\
\hline 52 & $20_{2,18}-19_{2,17}$ & 88890.385 & 5 & 45 & 256.2 & 21 & Blend with $\mathrm{CH}_{2}(\mathrm{OH}) \mathrm{CHO}$ and $U$-line \\
\hline 53 & $20_{1,19}-19_{1,18}$ & 89391.938 & 5 & 42 & 258.1 & 16 & Detected, blend with $\mathrm{CH}_{3} \mathrm{OCHO}, v_{t}=1$ \\
\hline 54 & $21_{1,21}-20_{1,20}$ & 91463.179 & 5 & 45 & 271.1 & 24 & Blend with $\mathrm{HC}_{3} \mathrm{~N}, v_{6}=v_{7}=1$ and $U$-line \\
\hline 55 & $21_{0,21}-20_{0,20}$ & 92164.912 & 5 & 44 & 271.6 & 27 & Detected, blend with $\mathrm{CH}_{3}{ }^{13} \mathrm{CN}, v_{8}=1$ and $U$-line? \\
\hline 56 & $21_{2,20}-20_{2,19}$ & 92714.159 & 5 & 49 & 269.2 & 28 & Blend with $\mathrm{CH}_{3} \mathrm{OCH}_{3}$ and $\mathrm{CH}_{3} \mathrm{CH}_{3} \mathrm{CO}, v_{t}=1$ \\
\hline 57 & $21_{6,16}-20_{6,15}{ }^{\star}$ & 92866.939 & 5 & 82 & 249.5 & 28 & Group detected, uncertain baseline \\
\hline 59 & $21_{7,14}-20_{7,13}^{\star}$ & 92867.332 & 5 & 95 & 241.5 & 28 & Group detected, uncertain baseline \\
\hline 61 & $21_{8,13}-20_{8,12}^{\star}$ & 92870.627 & 5 & 110 & 232.2 & 28 & Group detected, uncertain baseline \\
\hline 63 & $21_{5,17}-20_{5,16^{\star}}$ & 92871.289 & 5 & 70 & 256.3 & 28 & Group detected, uncertain baseline \\
\hline 65 & $21_{9,12}-20_{9,11}^{\star}$ & 92875.977 & 5 & 128 & 221.8 & 28 & Group detected, uncertain baseline \\
\hline 67 & 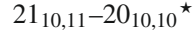 & 92882.945 & 5 & 147 & 210.1 & 28 & Blend with $\mathrm{CH}_{3} \mathrm{OCHO}$ \\
\hline 69 & $21_{4,18}-20_{4,17}$ & 92884.684 & 5 & 61 & 261.8 & 28 & Blend with $\mathrm{CH}_{3} \mathrm{OCHO}$ \\
\hline 70 & $21_{4,17}-20_{4,16}$ & 92885.520 & 5 & 61 & 261.8 & 28 & Blend with $\mathrm{CH}_{3} \mathrm{OCHO}$ \\
\hline 71 & $21_{11,10}-20_{11,9^{\star}}$ & 92891.291 & 6 & 169 & 197.1 & 28 & Noisy \\
\hline 73 & $21_{12,9}-20_{12,8^{\star}}$ & 92900.873 & 6 & 193 & 183.0 & 28 & Blend with $U$-line \\
\hline 75 & $21_{3,19}-20_{3,18}$ & 92904.745 & 5 & 54 & 266.1 & 28 & Blend with $\mathrm{CH}_{3} \mathrm{CH}_{3} \mathrm{CO}, v_{t}=1$ and $\mathrm{CH}_{3} \mathrm{CH}_{3} \mathrm{CO}$ \\
\hline 76 & $21_{3,18}-20_{3,17}$ & 92943.421 & 5 & 54 & 266.2 & 28 & Blend with $\mathrm{CH}_{3} \mathrm{OCHO}$ \\
\hline 77 & $21_{2,19}-20_{2,18}$ & 93376.934 & 5 & 49 & 269.2 & 22 & Detected \\
\hline 78 & $21_{1,20}-20_{1,19}$ & 93839.887 & 5 & 46 & 271.1 & 24 & Blend with $U$-line \\
\hline 79 & $22_{1,22}-21_{1,21}$ & 95802.965 & 5 & 49 & 284.0 & 23 & Blend with ${ }^{13} \mathrm{CH}_{3} \mathrm{CH}_{2} \mathrm{CN}$ and $\mathrm{C}_{2} \mathrm{H}_{5} \mathrm{CN}, v_{13}=1 / v_{21}=1$ \\
\hline 80 & $22_{0,22}-21_{0,21}$ & 96494.470 & 5 & 49 & 284.5 & 32 & Blend with $\mathrm{CH}_{3} \mathrm{OH}, v_{t}=1$ and $\mathrm{CH}_{3} \mathrm{CH}_{3} \mathrm{CO}$ \\
\hline
\end{tabular}


Table 9. continued.

\begin{tabular}{|c|c|c|c|c|c|c|c|}
\hline$N^{a}$ & Transition $^{b}$ & $\begin{array}{c}\text { Frequency } \\
\text { (MHz) } \\
\text { (3) }\end{array}$ & $\begin{array}{l}\text { Unc. }^{c} \\
(\mathrm{kHz}) \\
(4)\end{array}$ & $\begin{array}{l}E_{1}^{d} \\
(\mathrm{~K}) \\
(5)\end{array}$ & $\begin{array}{l}S \mu^{2} \\
\left(\mathrm{D}^{2}\right) \\
(6)\end{array}$ & $\begin{array}{l}\sigma^{e} \\
(\mathrm{mK}) \\
(7)\end{array}$ & Comments \\
\hline 81 & $22_{2,21}-21_{2,20}$ & 97115.607 & 5 & 53 & 282.3 & 21 & Blend with $U$-line \\
\hline 82 & $227,15-21_{7,14}$ & 97289.589 & 5 & 99 & 255.8 & 21 & Blend with $\mathrm{C}_{2} \mathrm{H}_{3} \mathrm{CN}$ and $\mathrm{OCS}$ \\
\hline 84 & $22_{6,17}-21_{6,16}{ }^{\star}$ & 97289.742 & 5 & 86 & 263.5 & 21 & Blend with $\mathrm{C}_{2} \mathrm{H}_{3} \mathrm{CN}$ and OCS \\
\hline 86 & $22_{8,14}-21_{8,13}{ }^{\star}$ & 97292.679 & 5 & 115 & 247.0 & 21 & Blend with OCS \\
\hline 88 & $22_{5,18}-21_{5,17}{ }^{\star}$ & 97295.253 & 5 & 75 & 269.9 & 21 & Strong OCS \\
\hline 90 & $22_{9,13}-21_{9,12}{ }^{\star}$ & 97298.036 & 5 & 132 & 237.0 & 21 & Strong OCS \\
\hline 92 & $22_{10,12}-21_{10,11^{\star}}$ & 97305.160 & 5 & 152 & 225.8 & 21 & Strong OCS \\
\hline 94 & $22_{4,19}-21_{4,18}$ & 97310.983 & 5 & 66 & 275.2 & 21 & Blend with $\mathrm{OCS}$ and $\mathrm{C}_{2} \mathrm{H}_{3} \mathrm{CN}, v_{11}=1$ \\
\hline 95 & $22_{4,18}-21_{4,17}$ & 97312.143 & 5 & 66 & 275.2 & 21 & Blend with $\mathrm{OCS}$ and $\mathrm{C}_{2} \mathrm{H}_{3} \mathrm{CN}, v_{11}=1$ \\
\hline 96 & $22_{11,11}-21_{11,10^{\star}}$ & 97313.773 & 6 & 173 & 213.5 & 21 & Blend with OCS and $\mathrm{C}_{2} \mathrm{H}_{3} \mathrm{CN}, v_{11}=1$ \\
\hline 98 & $22_{12,10}-21_{12,9^{\star}}$ & 97323.712 & 6 & 197 & 199.9 & 21 & Blend with $\mathrm{CH}_{3} \mathrm{OCHO}$ \\
\hline 100 & $22_{3,20}-21_{3,19}$ & 97332.446 & 5 & 58 & 279.4 & 21 & Blend with $U$-line \\
\hline 101 & $22_{13,9}-21_{13,8} \star$ & 97334.877 & 7 & 223 & 185.2 & 21 & Blend with $U$-line and $\mathrm{CH}_{3} \mathrm{OCHO}$ \\
\hline 103 & $22_{3,19}-21_{3,18}$ & 97381.167 & 5 & 58 & 279.4 & 20 & Blend with $\mathrm{CH}_{3} \mathrm{CH}_{3} \mathrm{CO}, v_{t}=1$, uncertain baseline \\
\hline 104 & $22_{2,20}-21_{2,19}$ & 97867.394 & 5 & 53 & 282.3 & 20 & Detected, uncertain baseline \\
\hline 105 & $22_{1,21}-21_{1,20}$ & 98284.291 & 5 & 51 & 284.0 & 18 & Blend with $\mathrm{CH}_{3} \mathrm{OCH}_{3}, \mathrm{CH}_{3} \mathrm{OCHO}$, and $U$-line \\
\hline 106 & $23_{1,23}-22_{1,22}$ & 100140.951 & 5 & 54 & 296.9 & 14 & Blend with $\mathrm{CH}_{3} \mathrm{OCHO}, v_{\mathrm{t}}=1$ and $\mathrm{HC}_{3} \mathrm{~N}, v_{5}=1 / v_{7}=3$ \\
\hline 107 & $23_{0,23}-22_{0,22}$ & 100818.358 & 5 & 53 & 297.4 & 20 & Blend with $\mathrm{CH}_{3}{ }^{13} \mathrm{CH}_{2} \mathrm{CN}$ and $U$-line \\
\hline 108 & $10_{4,6}-11_{3,9}$ & 101512.348 & 5 & 23 & 1.2 & 16 & Detected, small blend with $\mathrm{CH}_{3} \mathrm{CH}_{3} \mathrm{CO}, v_{t}=1$ \\
\hline 109 & $23_{2,22}-22_{2,21}$ & 101515.201 & 5 & 58 & 295.3 & 16 & Detected, small blend with $\mathrm{CH}_{3} \mathrm{CH}_{3} \mathrm{CO}, v_{t}=1$ \\
\hline 110 & $23_{7,16}-227,15^{\star}$ & 101711.846 & 5 & 104 & 270.0 & 16 & $\begin{array}{l}\text { Group detected, partial blend with }{ }^{13} \mathrm{CH}_{2} \mathrm{CHCN} \text {, uncertain } \\
\text { baseline }\end{array}$ \\
\hline 112 & $23_{6,18}-22_{6,17}{ }^{\star}$ & 101712.624 & 5 & 91 & 277.3 & 16 & $\begin{array}{l}\text { Group detected, partial blend with }{ }^{13} \mathrm{CH}_{2} \mathrm{CHCN} \text {, uncertain } \\
\text { baseline }\end{array}$ \\
\hline 114 & $23_{8,15}-22_{8,14}{ }^{\star}$ & 101714.680 & 5 & 120 & 261.5 & 16 & Group detected, uncertain baseline \\
\hline 116 & $23_{5,19}-22_{5,18}{ }^{\star}$ & 101719.429 & 5 & 79 & 283.5 & 16 & Group detected, small blend with ${ }^{13} \mathrm{CH}_{2} \mathrm{CHCN}$ \\
\hline 118 & $239,14-229,13$ * & 101720.011 & 5 & 137 & 252.0 & 16 & Group detected, small blend with ${ }^{13} \mathrm{CH}_{2} \mathrm{CHCN}$ \\
\hline 120 & $23_{10,13}-22_{10,12^{\star}}$ & 101727.266 & 5 & 157 & 241.3 & 16 & Blend with $\mathrm{CH}_{3} \mathrm{OCHO}$ and $U$-line? \\
\hline 122 & $23_{11,12-22_{11,11} \star}$ & 101736.128 & 6 & 178 & 229.5 & 16 & Blend with $\mathrm{CH}_{3} \mathrm{OH}$ \\
\hline 124 & $23_{4,20}-22_{4,19}$ & 101737.702 & 5 & 70 & 288.6 & 16 & Blend with $\mathrm{CH}_{3} \mathrm{OH}$ \\
\hline 125 & $23_{4,19}-22_{4,18}$ & 101739.288 & 5 & 70 & 288.6 & 16 & Blend with $\mathrm{CH}_{3} \mathrm{OH}$ \\
\hline 126 & $23_{12,11-22_{12,10}}{ }^{*}$ & 101746.410 & 6 & 202 & 216.6 & 16 & Blend with $U$-line and $\mathrm{C}_{2} \mathrm{H}_{5} \mathrm{CN}, v_{13}=1 / v_{21}=1$ \\
\hline 128 & $23_{13,10}-22_{13,9^{\star}}$ & 101757.997 & 7 & 227 & 202.5 & 16 & Blend with $U$-line \\
\hline 130 & $23_{3,21}-22_{3,20}$ & 101760.286 & 5 & 63 & 292.5 & 16 & Blend with $U$-line \\
\hline 131 & $23_{17,6^{-}}-22_{17,5^{\star}}$ & 101816.271 & 10 & 350 & 135.0 & 34 & Blend with $\mathrm{CH}_{3} \mathrm{CH}_{3} \mathrm{CO}, v_{t}=1$ and $v_{t}=0$, and $U$-line \\
\hline 133 & $23_{3,20}-22_{3,19}$ & 101820.997 & 5 & 63 & 292.5 & 34 & Blend with $\mathrm{CH}_{3} \mathrm{CH}_{3} \mathrm{CO}, v_{t}=1$ and $v_{t}=0$, and $U$-line \\
\hline 134 & $25_{3,22}-25_{2,23}$ & 102358.115 & 5 & 73 & 13.4 & 30 & Blend with $U$-line \\
\hline 135 & $23_{2,21}-22_{2,20}$ & 102361.359 & 5 & 58 & 295.3 & 30 & Blend with $U$-line \\
\hline 136 & $23_{1,22}-22_{1,21}$ & 102724.897 & 5 & 55 & 296.9 & 37 & Noisy, baseline problem \\
\hline 137 & $24_{1,24}-23_{1,23}$ & 104477.131 & 5 & 59 & 309.9 & 48 & Blend with $U$-line \\
\hline 138 & $24_{0,24}-23_{0,23}$ & 105136.938 & 5 & 58 & 310.3 & 28 & Blend with $U$-line \\
\hline 139 & $24_{2,23}-23_{2,22}$ & 105912.868 & 5 & 63 & 308.3 & 43 & Blend with ${ }^{13} \mathrm{CH}_{3} \mathrm{OH}$ \\
\hline 140 & $24_{7,17}-23_{7,16}{ }^{\star}$ & 106134.103 & 5 & 109 & 284.1 & 25 & Blend with $\mathrm{NH}_{2} \mathrm{CHO}$ \\
\hline 142 & $37_{2,36}-37_{1,37}$ & 106135.161 & 50 & 148 & 12.1 & 25 & Blend with $\mathrm{NH}_{2} \mathrm{CHO}$ \\
\hline 143 & $24_{6,19}-23_{6,18}{ }^{\star}$ & 106135.587 & 5 & 96 & 291.1 & 25 & Blend with $\mathrm{NH}_{2} \mathrm{CHO}$ \\
\hline 145 & $24_{8,16}-23_{8,15}^{\star}$ & 106136.628 & 5 & 124 & 276.0 & 25 & Blend with $\mathrm{NH}_{2} \mathrm{CHO}$ \\
\hline 147 & $24_{9,15}-23_{9,14^{\star}}$ & 106141.897 & 5 & 142 & 266.9 & 25 & Blend with $\mathrm{NH}_{2} \mathrm{CHO}$ \\
\hline 149 & $24_{5,20}-23_{5,19}{ }^{\star}$ & 106143.824 & 5 & 84 & 297.0 & 25 & Blend with $\mathrm{NH}_{2} \mathrm{CHO}$ and $\mathrm{C}_{2} \mathrm{H}_{5} \mathrm{CN}, v_{13}=1 / v_{21}=1$ \\
\hline 151 & $24_{10,14}-23_{10,13}{ }^{\star}$ & 106149.258 & 5 & 161 & 256.6 & 25 & Blend with $\mathrm{C}_{2} \mathrm{H}_{5} \mathrm{CN}, v_{13}=1 / v_{21}=1$ \\
\hline 153 & $24_{11,13}-23_{11,12}{ }^{\star}$ & 106158.350 & 6 & 183 & 245.3 & 25 & Blend with $\mathrm{CH}_{3} \mathrm{CH}_{3} \mathrm{CO}$ and $\mathrm{CH}_{2} \mathrm{CH}^{13} \mathrm{CN}$ \\
\hline 155 & $24_{4,21}-23_{4,20}$ & 106164.853 & 5 & 75 & 301.9 & 25 & Blend with $\mathrm{CH}_{2} \mathrm{CH}^{13} \mathrm{CN}$ and $\mathrm{C}_{2} \mathrm{H}_{5} \mathrm{CN}, v_{13}=1 / v_{21}=1$ \\
\hline 156 & $24_{4,20}-23_{4,19}$ & 106166.991 & 5 & 75 & 301.9 & 25 & Blend with $\mathrm{CH}_{2} \mathrm{CH}^{13} \mathrm{CN}$ and $\mathrm{C}_{2} \mathrm{H}_{5} \mathrm{CN}, v_{13}=1 / v_{21}=1$ \\
\hline 157 & $24_{12,12}-23_{12,11}{ }^{\star}$ & 106168.961 & 6 & 207 & 232.9 & 25 & Blend with $\mathrm{C}_{2} \mathrm{H}_{5} \mathrm{CN}, v_{13}=1 / v_{21}=1$ \\
\hline 159 & $24_{13,11}-23_{13,10^{\star}}$ & 106180.959 & 7 & 232 & 219.4 & 25 & Blend with $\mathrm{C}_{2} \mathrm{H}_{5} \mathrm{CN}, v_{13}=1 / v_{21}=1$ \\
\hline 161 & $24_{3,22}-23_{3,21}$ & 106188.197 & 5 & 68 & 305.7 & 25 & Detected, noisy \\
\hline 162 & $24_{14,10}-23_{14,9^{\star}}$ & 106194.262 & 7 & 260 & 204.8 & 25 & Blend with $\mathrm{CH}_{3} \mathrm{CH}_{3} \mathrm{CO}, v_{t}=1$ and $U$-line \\
\hline 164 & $24_{18,6}-23_{18,5}$ * & 106259.676 & 12 & 391 & 135.8 & 25 & Blend with ${ }^{13} \mathrm{CH}_{3} \mathrm{CH}_{2} \mathrm{CN}$ \\
\hline 166 & $24_{3,21}-23_{3,20}$ & 106263.099 & 5 & 68 & 305.6 & 25 & Blend with ${ }^{13} \mathrm{CH}_{3} \mathrm{CH}_{2} \mathrm{CN}$ and $U$-line \\
\hline 167 & $24_{2,22}-23_{2,21}$ & 106858.357 & 5 & 63 & 308.4 & 24 & Blend with $U$-line, $\mathrm{C}_{2} \mathrm{H}_{5} \mathrm{OH}$, and $\mathrm{CH}_{3} \mathrm{CH}_{3} \mathrm{CO}, v_{t}=1$ \\
\hline 168 & $24_{1,23}-23_{1,22}$ & 107161.444 & 5 & 60 & 309.9 & 24 & Blend with $\mathrm{CH}_{3} \mathrm{OH}$ and ${ }^{13} \mathrm{CH}_{3} \mathrm{CN}$ \\
\hline 169 & $25_{1,25}-24_{1,24}$ & 108811.506 & 5 & 64 & 322.9 & 20 & Blend with ${ }^{13} \mathrm{CN}$ in absorption and $\mathrm{C}_{2} \mathrm{H}_{3} \mathrm{CN}$ \\
\hline 170 & $25_{0,25}-24_{0,24}$ & 109450.626 & 5 & 63 & 323.2 & 36 & Blend with $\mathrm{HC}_{3} \mathrm{~N}, v_{7}=1$ and $\mathrm{OCS}$ \\
\hline 171 & $78_{4,74-78_{3,75}}$ & 109452.759 & 321 & 668 & 59.9 & 36 & Blend with $\mathrm{HC}_{3} \mathrm{~N}, v_{7}=1$ and $\mathrm{OCS}$ \\
\hline 172 & $25_{2,24}-24_{2,23}$ & 110308.536 & 5 & 68 & 321.3 & 24 & Strong $\mathrm{CH}_{3}{ }^{13} \mathrm{CN}$ \\
\hline
\end{tabular}


Table 9. continued.

\begin{tabular}{|c|c|c|c|c|c|c|c|}
\hline$N^{a}$ & Transition $^{b}$ & $\begin{array}{c}\text { Frequency } \\
\text { (MHz) } \\
\text { (3) }\end{array}$ & $\begin{array}{l}\text { Unc. }^{c} \\
(\mathrm{kHz}) \\
(4)\end{array}$ & $\begin{array}{l}E_{1}^{d} \\
(\mathrm{~K}) \\
(5)\end{array}$ & $\begin{array}{l}S \mu^{2} \\
\left(\mathrm{D}^{2}\right) \\
(6)\end{array}$ & $\begin{array}{c}\sigma^{e} \\
(\mathrm{mK}) \\
(7)\end{array}$ & Comments \\
\hline 173 & $25_{7,18}-24_{7,17^{\star}}$ & 110556.359 & 5 & 114 & 298.1 & 32 & Blend with $U$-lines and $\mathrm{CH}_{3} \mathrm{OCHO}$ \\
\hline 175 & $25_{8,17}-24_{8,16}^{\star}$ & 110558.521 & 5 & 130 & 290.3 & 32 & Blend with $U$-lines and $\mathrm{CH}_{3} \mathrm{OCHO}$ \\
\hline 177 & $25_{6,20}-24_{6,19}{ }^{\star}$ & 110558.636 & 5 & 101 & 304.8 & 32 & Blend with $U$-lines and $\mathrm{CH}_{3} \mathrm{OCHO}$ \\
\hline 179 & $25_{9,16}-24_{9,15}{ }^{\star}$ & 110563.690 & 5 & 147 & 281.5 & 32 & Blend with $U$-line \\
\hline 181 & $25_{5,21}-24_{5,20}{ }^{\star}$ & 110568.450 & 5 & 89 & 310.5 & 32 & Blend with $\mathrm{CH}_{3} \mathrm{OCHO}, v_{\mathrm{t}}=1$ \\
\hline 183 & 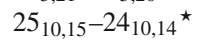 & 110571.130 & 6 & 167 & 271.7 & 32 & Blend with $\mathrm{CH}_{3} \mathrm{OCHO}, v_{\mathrm{t}}=1$ \\
\hline 185 & $25_{11,14}-24_{11,13}{ }^{\star}$ & 110580.433 & 6 & 188 & 260.8 & 32 & Blend with $\mathrm{CH}_{3} \mathrm{CH}_{3} \mathrm{CO}$ and $U$-line, uncertain baseline \\
\hline 187 & $86_{6,80}-85_{7,79}$ & 110581.350 & 563 & 826 & 15.9 & 32 & Blend with $\mathrm{CH}_{3} \mathrm{CH}_{3} \mathrm{CO}$ and $U$-line, uncertain baseline \\
\hline 188 & $25_{12,13}-24_{12,12^{\star}}$ & 110591.357 & 6 & 212 & 248.9 & 32 & Blend with $U$-line, $\mathrm{CH}_{3}{ }^{13} \mathrm{CN}, v_{8}=1$, and $\mathrm{HNCO}, v_{4}=1$ \\
\hline 190 & $25_{4,22}-24_{4,21}$ & 110592.442 & 5 & 80 & 315.2 & 32 & Blend with $U$-line, $\mathrm{CH}_{3}{ }^{13} \mathrm{CN}, v_{8}=1$, and $\mathrm{HNCO}, v_{4}=1$ \\
\hline 191 & $28_{3,26}-28_{2,27}$ & 110593.409 & 5 & 90 & 14.3 & 32 & Blend with $U$-line, $\mathrm{CH}_{3}{ }^{13} \mathrm{CN}, v_{8}=1$, and $\mathrm{HNCO}, v_{4}=1$ \\
\hline 192 & $25_{4,21}-24_{4,20}$ & 110595.288 & 5 & 80 & 315.1 & 32 & Blend with $\mathrm{CH}_{3}{ }^{13} \mathrm{CN}, v_{8}=1$ and $\mathrm{HNCO}, v_{4}=1$ \\
\hline 193 & $46_{1,45}-46_{1,46}$ & 110603.457 & 123 & 227 & 0.8 & 32 & Blend with $\mathrm{CH}_{3} \mathrm{CN}, v_{8}=1$ and $U$-line \\
\hline 194 & $25_{13,12}-24_{13,11}$ * & 110603.755 & 7 & 237 & 236.0 & 32 & Blend with $\mathrm{CH}_{3} \mathrm{CN}, v_{8}=1$ and $U$-line \\
\hline 196 & $25_{3,23}-24_{3,22}$ & 110616.104 & 5 & 73 & 318.8 & 32 & Blend with $\mathrm{CH}_{3} \mathrm{CN}, v_{8}=1$ and $\mathrm{CH}_{3}{ }^{13} \mathrm{CN}, v_{8}=1$ \\
\hline 197 & 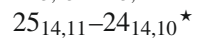 & 110617.532 & 7 & 265 & 222.0 & 32 & Blend with $\mathrm{CH}_{3} \mathrm{CN}, v_{8}=1$ and $\mathrm{CH}_{3}{ }^{13} \mathrm{CN}, v_{8}=1$ \\
\hline 199 & $25_{15,10}-24_{15,9^{\star}}$ & 110632.625 & 8 & 295 & 207.0 & 32 & Blend with $\mathrm{CH}_{3}{ }^{13} \mathrm{CN}, v_{8}=1$ and $\mathrm{CH}_{3} \mathrm{CN}, v_{8}=1$ \\
\hline 201 & $25_{19,6}-24_{19,5}{ }^{\star}$ & 110705.543 & 14 & 434 & 136.6 & 32 & Strong $\mathrm{CH}_{3} \mathrm{CN}, v_{8}=1$ \\
\hline 203 & $25_{3,22}-24_{3,21}$ & 110707.662 & 5 & 73 & 318.8 & 32 & Strong $\mathrm{CH}_{3} \mathrm{CN}, v_{8}=1$ \\
\hline 204 & $25_{2,23}-24_{2,22}$ & 111357.863 & 5 & 68 & 321.4 & 35 & Noisy \\
\hline 205 & $25_{1,24}-24_{1,23}$ & 111593.662 & 5 & 65 & 322.9 & 29 & Detected \\
\hline 206 & $26_{1,26}-25_{1,25}$ & 113144.084 & 5 & 69 & 335.9 & 40 & Strong $\mathrm{CN}$ in absorption \\
\hline 207 & $26_{0,26}-25_{0,25}$ & 113759.873 & 5 & 69 & 336.1 & 34 & Blend with $\mathrm{CH}_{3} \mathrm{OCHO}$ and $U$-line \\
\hline 208 & $26_{2,25}-25_{2,24}$ & 114702.134 & 5 & 73 & 334.4 & 37 & Blend with $\mathrm{CH}_{3} \mathrm{OCH}_{3}$ \\
\hline 209 & $26_{7,19}-25_{7,18}^{\star}$ & 114978.615 & 5 & 119 & 312.0 & 59 & Blend with $\mathrm{C}_{2} \mathrm{H}_{3} \mathrm{CN}, v_{11}=3$ and ${ }^{13} \mathrm{CH}_{3} \mathrm{OH}$ \\
\hline 211 & $26_{8,18}-25_{8,17^{\star}}$ & 114980.356 & 5 & 135 & 304.5 & 59 & Blend with $\mathrm{C}_{2} \mathrm{H}_{3} \mathrm{CN}, v_{11}=3$ and ${ }^{13} \mathrm{CH}_{3} \mathrm{OH}$ \\
\hline 213 & $26_{6,21}-25_{6,20} \star$ & 114981.774 & 5 & 106 & 318.5 & 59 & Blend with $\mathrm{C}_{2} \mathrm{H}_{3} \mathrm{CN}, v_{11}=3,{ }^{13} \mathrm{CH}_{3} \mathrm{OH}$, and $\mathrm{C}_{2} \mathrm{H}_{3} \mathrm{CN}, v_{11}=3$ \\
\hline 215 & $269,17-25_{9,16}{ }^{\star}$ & 114985.387 & 5 & 152 & 296.1 & 59 & Blend with $\mathrm{C}_{2} \mathrm{H}_{3} \mathrm{CN}, v_{11}=3$ and $\mathrm{CH}_{3} \mathrm{CH}_{3} \mathrm{CO}$ \\
\hline 217 & $26_{10,16}-25_{10,15}$ * & 114992.878 & 6 & 172 & 286.6 & 59 & Blend with $\mathrm{CH}_{3} \mathrm{CH}_{3} \mathrm{CO}$ \\
\hline 219 & $26_{5,22}-25_{5,21} \star$ & 114993.314 & 5 & 95 & 323.9 & 59 & Blend with $\mathrm{CH}_{3} \mathrm{CH}_{3} \mathrm{CO}$ \\
\hline 221 & 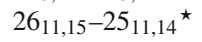 & 115002.371 & 6 & 193 & 276.2 & 59 & Blend with $\mathrm{C}_{2} \mathrm{H}_{5} \mathrm{CN}, v_{13}=1 / v_{21}=1$ and $\mathrm{C}_{2} \mathrm{H}_{3} \mathrm{CN}, v_{11}=1$ \\
\hline 223 & $26_{12,14}-25_{12,13}{ }^{\star}$ & 115013.593 & 6 & 217 & 264.7 & 59 & Blend with $\mathrm{C}_{2} \mathrm{H}_{5} \mathrm{CN}, v_{13}=1 / v_{21}=1, \mathrm{CH}_{2}{ }^{13} \mathrm{CHCN}$, and $U$-line \\
\hline 225 & $26_{4,23}-25_{4,22}$ & 115020.476 & 5 & 85 & 328.4 & 59 & Blend with $U$-line \\
\hline 226 & $26_{4,22}-25_{4,21}$ & 115024.221 & 5 & 85 & 328.4 & 59 & Blend with $U$-line \\
\hline 227 & $26_{13,13}-25_{13,12^{\star}}$ & 115026.378 & 7 & 243 & 252.3 & 59 & Blend with $U$-line and $\mathrm{C}_{2} \mathrm{H}_{5} \mathrm{OH}$ \\
\hline 229 & $26_{14,12}-25_{14,11}{ }^{\star}$ & 115040.619 & 8 & 270 & 238.9 & 59 & Blend with $U$-line \\
\hline 231 & $26_{3,24}-25_{3,23}$ & 115043.924 & 5 & 78 & 331.9 & 59 & Blend with $U$-line \\
\hline 232 & $26_{15,11}-25_{15,10}{ }^{\star}$ & 115056.245 & 8 & 300 & 224.4 & 59 & Blend with $\mathrm{H}^{13} \mathrm{CCCN}, v_{7}=1$ \\
\hline 234 & $64_{4,60}-64_{3,61}$ & 115150.722 & 157 & 453 & 43.9 & 59 & $\begin{array}{l}\text { Blend with } \mathrm{C}_{2} \mathrm{H}_{3} \mathrm{CN}, \mathrm{CH}_{3} \mathrm{OCHO}, v_{\mathrm{t}}=1, \mathrm{NS} \text {, and } \\
\mathrm{CH}_{3} \mathrm{CH}_{3} \mathrm{CO}, v_{t}=1\end{array}$ \\
\hline 235 & $26_{20,6}-25_{20,5}{ }^{\star}$ & 115154.006 & 17 & 479 & 137.3 & 59 & Blend with $\mathrm{CH}_{3} \mathrm{OCHO}, v_{\mathrm{t}}=1, \mathrm{NS}$, and $\mathrm{CH}_{3} \mathrm{CH}_{3} \mathrm{CO}, v_{t}=1$ \\
\hline 237 & $26_{3,23}-25_{3,22}$ & 115154.885 & 5 & 78 & 331.9 & 59 & Blend with $\mathrm{CH}_{3} \mathrm{OCHO}, v_{\mathrm{t}}=1, \mathrm{NS}$, and $\mathrm{CH}_{3} \mathrm{CH}_{3} \mathrm{CO}, v_{t}=1$ \\
\hline 238 & $26_{2,24}-25_{2,23}$ & 115859.309 & 5 & 73 & 334.4 & 79 & Weak, blend with $\mathrm{a}\left(\mathrm{CH}_{2} \mathrm{OH}\right)_{2}$ and $\mathrm{C}_{2} \mathrm{H}_{5} \mathrm{OH}$ \\
\hline 239 & $33_{2,32-32,31}$ & 145394.105 & 5 & 116 & 425.3 & 25 & Blend with $U$-line and $\mathrm{NH}_{2} \mathrm{CH}_{2} \mathrm{CN}$ \\
\hline 240 & $34_{1,34}-33_{1,33}$ & 147742.893 & 6 & 118 & 439.5 & 31 & Partial blend with $\mathrm{C}_{2} \mathrm{H}_{5} \mathrm{CN}$ and $\mathrm{CH}_{3} \mathrm{CN}, v_{8}=1$, uncertain baseline \\
\hline 241 & $37_{8,29}-36_{8,28}{ }^{\star}$ & 163616.044 & 6 & 207 & 456.4 & 38 & Blend with $\mathrm{SO}_{2}$ \\
\hline 243 & $37_{9,28}-369,27^{\star}$ & 163616.543 & 6 & 225 & 450.4 & 38 & Blend with $\mathrm{SO}_{2}$ \\
\hline 245 & $65_{4,62}-65_{3,63}$ & 163620.536 & 185 & 464 & 33.9 & 38 & Blend with $\mathrm{SO}_{2}$ \\
\hline 246 & $37_{10,27}-36_{10,26}$ 夫 & 163622.456 & 6 & 244 & 443.8 & 38 & Blend with $U$-line, uncertain baseline \\
\hline 248 & $37_{7,31}-36_{7,30}{ }^{\star}$ & 163623.355 & 5 & 192 & 461.6 & 38 & Blend with $U$-line, uncertain baseline \\
\hline 250 & $37_{11,26}-36_{11,25}$ * & 163632.454 & 7 & 266 & 436.4 & 38 & Blend with $\mathrm{NH}_{2} \mathrm{CH}_{2} \mathrm{CN}$, uncertain baseline \\
\hline 252 & $37_{6,32}-36_{6,31}{ }^{\star}$ & 163643.143 & 5 & 178 & 466.1 & 38 & Blend with $\mathrm{NH}_{2} \mathrm{CH}_{2} \mathrm{CN}$, uncertain baseline \\
\hline 254 & $37_{12,25}-36_{12,24}^{\star}$ & 163645.748 & 7 & 289 & 428.3 & 38 & Blend with $\mathrm{NH}_{2} \mathrm{CH}_{2} \mathrm{CN}$, uncertain baseline \\
\hline 256 & $37_{13,24}-36_{13,23}{ }^{\star}$ & 163661.850 & 8 & 315 & 419.6 & 38 & Blend with $\mathrm{C}_{2} \mathrm{H}_{5} \mathrm{OCHO}$, uncertain baseline \\
\hline 258 & $37_{14,23}-36_{14,22}{ }^{\star}$ & 163680.443 & 8 & 343 & 410.2 & 38 & Blend with $\mathrm{C}_{2} \mathrm{H}_{5} \mathrm{CN}, v_{13}=1 / v_{21}=1$ and $\mathrm{HC}^{13} \mathrm{CCN}, v_{7}=1$ \\
\hline 260 & $37_{5,33}-36_{5,32}$ & 163684.984 & 5 & 167 & 470.0 & 38 & Blend with $\mathrm{HC}^{13} \mathrm{CCN}, v_{7}=1$ and $\mathrm{C}_{2} \mathrm{H}_{5} \mathrm{CN}$ \\
\hline 261 & $37_{5,32}-36_{5,31}$ & 163686.545 & 5 & 167 & 470.0 & 38 & Blend with $\mathrm{HC}^{13} \mathrm{CCN}, v_{7}=1$ and $\mathrm{C}_{2} \mathrm{H}_{5} \mathrm{CN}$ \\
\hline 262 & $37_{15,22}-36_{15,21}$ * & 163701.318 & 9 & 373 & 400.1 & 38 & Blend with $\mathrm{HC}^{13} \mathrm{CCN}, v_{7}=1$ \\
\hline 264 & $37_{3,35}-36_{3,34}$ & 163715.634 & 5 & 151 & 475.5 & 38 & Blend with $\mathrm{CH}_{3} \mathrm{CH}_{3} \mathrm{CO}, v_{t}=1$ \\
\hline 265 & $37_{16,21}-36_{16,20}$ * & 163724.332 & 9 & 404 & 389.2 & 38 & Blend with g- $\mathrm{C}_{3} \mathrm{H}_{7} \mathrm{CN}$ and $U$-line \\
\hline 267 & $39_{9,30}-38_{9,29}{ }^{\star}$ & 172456.851 & 6 & 241 & 477.7 & 44 & Strong $\mathrm{C}_{2} \mathrm{H}_{5} \mathrm{CN}$ and $\mathrm{CH}_{3} \mathrm{OCHO}, v_{\mathrm{t}}=1$ \\
\hline 269 & $39_{8,31}-38_{8,30} \star$ & 172457.861 & 6 & 223 & 483.3 & 44 & Strong $\mathrm{C}_{2} \mathrm{H}_{5} \mathrm{CN}$ and $\mathrm{CH}_{3} \mathrm{OCHO}, v_{\mathrm{t}}=1$ \\
\hline 271 & $39_{10,29}-38_{10,28}{ }^{\star}$ & 172461.990 & 7 & 260 & 471.4 & 44 & Strong $\mathrm{C}_{2} \mathrm{H}_{5} \mathrm{CN}$ and $\mathrm{CH}_{3} \mathrm{OCHO}, v_{\mathrm{t}}=1$ \\
\hline 273 & $39_{7,33}-38_{7,32^{\star}}$ & 172467.829 & 6 & 208 & 488.4 & 44 & Strong $\mathrm{CH}_{3} \mathrm{OCHO}, v_{\mathrm{t}}=1$ and $\mathrm{C}_{2} \mathrm{H}_{3} \mathrm{CN}$ \\
\hline
\end{tabular}


Table 9. continued.

\begin{tabular}{|c|c|c|c|c|c|c|c|}
\hline$N^{a}$ & Transition $^{b}$ & $\begin{array}{c}\text { Frequency } \\
(\mathrm{MHz}) \\
\text { (3) }\end{array}$ & $\begin{array}{c}\text { Unc. }^{c} \\
(\mathrm{kHz}) \\
(4)\end{array}$ & $\begin{array}{l}E_{1}^{d} \\
(\mathrm{~K}) \\
(5)\end{array}$ & $\begin{array}{l}S \mu^{2} \\
\left(\mathrm{D}^{2}\right) \\
(6)\end{array}$ & $\begin{array}{c}\sigma^{e} \\
(\mathrm{mK}) \\
(7)\end{array}$ & Comments \\
\hline 275 & $39_{11,28}-38_{11,27^{\star}}$ & 172471.720 & 7 & 282 & 464.4 & 44 & Strong $\mathrm{CH}_{3} \mathrm{OCHO}, v_{\mathrm{t}}=1$ and $\mathrm{C}_{2} \mathrm{H}_{3} \mathrm{CN}$ \\
\hline 277 & $39_{12,27}-38_{12,26}$ * & 172485.116 & 8 & 305 & 456.8 & 44 & Blend with $\mathrm{C}_{2} \mathrm{H}_{5} \mathrm{CN}$ \\
\hline 279 & $39_{6,34}-38_{6,33^{\star}}$ & 172492.223 & 5 & 194 & 492.7 & 44 & Blend with $\mathrm{C}_{2} \mathrm{H}_{5} \mathrm{CN}$ \\
\hline 281 & $39_{13,26}-38_{13,25}{ }^{\star}$ & 172501.606 & 8 & 331 & 448.5 & 44 & Blend with $\mathrm{C}_{2} \mathrm{H}_{5} \mathrm{CN}, v_{13}=1 / v_{21}=1$ \\
\hline 283 & $52_{2,50-51_{3,49}}$ & 172516.550 & 77 & 291 & 14.0 & 44 & Blend with $\mathrm{C}_{2} \mathrm{H}_{5} \mathrm{CN}, v_{13}=1 / v_{21}=1$ and $\mathrm{CH}_{3} \mathrm{OCHO}, v_{\mathrm{t}}=1$ \\
\hline 284 & $39_{14,25}-38_{14,24}{ }^{\star}$ & 172520.819 & 8 & 359 & 439.6 & 44 & $\begin{array}{l}\text { Blend with } \mathrm{CH}_{3} \mathrm{OCHO}, v_{\mathrm{t}}=1, \mathrm{HC}^{13} \mathrm{CCN}, v_{7}=1 \text {, } \\
\text { and } \mathrm{HC}^{13} \mathrm{CCN}, v_{6}=1\end{array}$ \\
\hline 286 & $39_{5,35}-38_{5,34}$ & 172542.025 & 5 & 183 & 496.3 & 44 & Blend with $\mathrm{NH}_{2} \mathrm{CH}_{2} \mathrm{CN}$ and $\mathrm{HCC}^{13} \mathrm{CN}, v_{6}=1$ \\
\hline 287 & $39_{15,24}-38_{15,23}{ }^{\star}$ & 172542.509 & 9 & 388 & 429.9 & 44 & Blend with $\mathrm{NH}_{2} \mathrm{CH}_{2} \mathrm{CN}$ and $\mathrm{HCC}^{13} \mathrm{CN}, v_{6}=1$ \\
\hline 289 & $39_{5,34}-38_{5,33}$ & 172544.526 & 5 & 183 & 496.3 & 44 & Blend with $\mathrm{HCC}^{13} \mathrm{CN}, v_{6}=1$ and $\mathrm{HCC}^{13} \mathrm{CN}, v_{7}=1$ \\
\hline 290 & $39,37-38_{3,36}$ & 172551.469 & 5 & 167 & 501.6 & 44 & Blend with $\mathrm{HCC}^{13} \mathrm{CN}, v_{6}=1$ and $\mathrm{HCC}^{13} \mathrm{CN}, v_{7}=1$ \\
\hline 291 & $39_{16,23}-38_{16,22}{ }^{\star}$ & 172566.508 & 9 & 420 & 419.6 & 44 & Blend with $\mathrm{NH}_{2} \mathrm{CH}_{2} \mathrm{CN}$ \\
\hline 293 & $39_{17,22}-38_{17,21}$ * & 172592.700 & 10 & 454 & 408.7 & 44 & Blend with $\mathrm{CH}_{2}(\mathrm{OH}) \mathrm{CHO}$ and $U$-line \\
\hline 295 & $39_{18,21}-38_{18,20}$ * & 172621.005 & 11 & 490 & 397.1 & 44 & Blend with $\mathrm{NH}_{2} \mathrm{CH}_{2} \mathrm{CN}$, t- $\mathrm{HCOOH}$, and $U$-line? \\
\hline 297 & $39_{4,36}-38_{4,35}$ & 172622.501 & 5 & 174 & 499.2 & 44 & Blend with $\mathrm{NH}_{2} \mathrm{CH}_{2} \mathrm{CN}$, t- $\mathrm{HCOOH}$, and $U$-line? \\
\hline 298 & $39_{19,20}-38_{19,19}{ }^{\star}$ & 172651.366 & 13 & 528 & 384.8 & 44 & Blend with $\mathrm{C}_{2} \mathrm{H}_{5} \mathrm{CN}, v_{20}=1$ and $U$-line \\
\hline 300 & $39_{20,19}-38_{20,18^{\star}}$ & 172683.745 & 16 & 568 & 371.8 & 44 & Strong $\mathrm{H}^{13} \mathrm{CN}$ in absorption and $\mathrm{CH}_{3} \mathrm{OCHO}$ \\
\hline 302 & $39_{4,35}-38_{4,34}$ & 172684.518 & 5 & 174 & 499.2 & 44 & Strong $\mathrm{H}^{13} \mathrm{CN}$ in absorption and $\mathrm{CH}_{3} \mathrm{OCHO}$ \\
\hline 303 & $46_{2,45}-45_{2,44}$ & 202062.851 & 7 & 223 & 593.9 & 138 & Blend with $\mathrm{CH}_{3}{ }^{13} \mathrm{CN}$ \\
\hline 304 & $46_{1,45}-45_{1,44}$ & 203271.058 & 6 & 222 & 594.2 & 161 & Strong $\mathrm{H}^{13} \mathrm{CCCN}, v_{7}=1$ and $\mathrm{C}_{2} \mathrm{H}_{3} \mathrm{CN}, v_{11}=1$ \\
\hline 305 & $46_{9,37}-45_{9,36}{ }^{\star}$ & 203392.918 & 7 & 303 & 572.3 & 161 & Strong $\mathrm{SO}_{2}$ and $\mathrm{C}_{2} \mathrm{H}_{5} \mathrm{CN}$ \\
\hline 307 & $46_{10,36}-45_{10,35}{ }^{\star}$ & 203393.942 & 8 & 323 & 567.0 & 161 & Strong $\mathrm{SO}_{2}$ and $\mathrm{C}_{2} \mathrm{H}_{5} \mathrm{CN}$ \\
\hline 309 & $46_{8,39}-45_{8,38^{\star}}$ & 203401.192 & 7 & 286 & 577.1 & 161 & Strong $\mathrm{C}_{2} \mathrm{H}_{5} \mathrm{CN}$ and $\mathrm{CH}_{3} \mathrm{OCH}_{3}$ \\
\hline 311 & 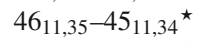 & 203401.696 & 8 & 344 & 561.1 & 161 & Strong $\mathrm{C}_{2} \mathrm{H}_{5} \mathrm{CN}$ and $\mathrm{CH}_{3} \mathrm{OCH}_{3}$ \\
\hline 313 & $46_{12,34}-45_{12,33^{\star}}$ & 203414.661 & 9 & 368 & 554.6 & 161 & Strong $\mathrm{CH}_{3} \mathrm{OCH}_{3}$ and ${ }^{13} \mathrm{CH}_{2} \mathrm{CHCN}$ \\
\hline 315 & $46_{3,44}-45_{3,43}$ & 203419.779 & 6 & 229 & 592.5 & 161 & Blend with ${ }^{13} \mathrm{CH}_{2} \mathrm{CHCN}$ \\
\hline 316 & $46_{7,40}-45_{7,39}{ }^{\star}$ & 203423.393 & 6 & 270 & 581.4 & 161 & Blend with ${ }^{13} \mathrm{CH}_{2} \mathrm{CHCN}$ \\
\hline 318 & $46_{13,33}-45_{13,32}{ }^{\star}$ & 203431.894 & 9 & 393 & 547.6 & 161 & Blend with $\mathrm{CH}_{3} \mathrm{CN}, v_{8}=2$ \\
\hline 320 & $46_{14,32}-45_{14,31}$ 太 & 203452.784 & 9 & 421 & 540.0 & 161 & Blend with $\mathrm{CH}_{3} \mathrm{CN}, v_{8}=2$ \\
\hline 322 & $46_{6,41}-45_{6,40}$ & 203468.489 & 6 & 257 & 585.0 & 161 & Blend with $\mathrm{CH}_{3} \mathrm{CN}, v_{8}=2$ and $\mathrm{CH}_{3} \mathrm{OCHO}$ \\
\hline 323 & $46_{6,40}-45_{6,39}$ & 203468.859 & 6 & 257 & 585.0 & 161 & Blend with $\mathrm{CH}_{3} \mathrm{CN}, v_{8}=2$ and $\mathrm{CH}_{3} \mathrm{OCHO}$ \\
\hline 324 & $46_{15,31}-45_{15,30}{ }^{\star}$ & 203476.924 & 9 & 451 & 531.9 & 161 & Blend with $\mathrm{CH}_{3} \mathrm{OCHO}$ and $\mathrm{CH}_{3} \mathrm{CN}, v_{8}=2$ \\
\hline 326 & $46_{16,30}-45_{16,29}{ }^{\star}$ & 203504.034 & 9 & 483 & 523.1 & 161 & Blend with ${ }^{34} \mathrm{SO}_{2}$ and $\mathrm{CH}_{3} \mathrm{CN}, v_{8}=2$ \\
\hline 328 & $46_{17,29}-45_{17,28} \star$ & 203533.921 & 10 & 517 & 513.8 & 161 & Blend with $\mathrm{CH}_{3} \mathrm{CN}, v_{8}=2$ \\
\hline 330 & $16_{7,9}-17_{6,12}^{\star}$ & 203535.308 & 25 & 70 & 1.6 & 161 & Blend with $\mathrm{CH}_{3} \mathrm{CN}, v_{8}=2$ and $\mathrm{C}_{2} \mathrm{H}_{5} \mathrm{OCHO}$ \\
\hline 332 & $46_{5,42}-45_{5,41}$ & 203552.152 & 6 & 245 & 588.1 & 161 & Blend with $\mathrm{CH}_{3} \mathrm{CN}, v_{8}=2$ and $\mathrm{H}^{13} \mathrm{CCCN}, v_{7}=1$ \\
\hline 333 & $46_{5,41}-45_{5,40}$ & 203562.997 & 6 & 245 & 588.2 & 161 & Strong $\mathrm{CH}_{3} \mathrm{CN}, v_{8}=2, \mathrm{CH}_{3} \mathrm{OCH}_{3}$, and ${ }^{13} \mathrm{CH}_{2} \mathrm{CHCN}$ \\
\hline 334 & $46_{18,28}-45_{18,27}{ }^{\star}$ & 203566.446 & 10 & 552 & 504.0 & 161 & Strong $\mathrm{CH}_{3} \mathrm{CN}, v_{8}=2, \mathrm{CH}_{3} \mathrm{OCH}_{3}$, and ${ }^{13} \mathrm{CH}_{2} \mathrm{CHCN}$ \\
\hline 336 & $46_{19,27}-45_{19,26}$ * & 203601.513 & 11 & 590 & 493.6 & 161 & Blend with $\mathrm{CH}_{3} \mathrm{OCHO}$ and $\mathrm{CH}_{3} \mathrm{CH}_{3} \mathrm{CO}$ \\
\hline 338 & $46_{20,26}-45_{20,25}$ * & 203639.052 & 14 & 630 & 482.7 & 161 & Blend with $\mathrm{C}_{2} \mathrm{H}_{5} \mathrm{CN}$ \\
\hline 340 & $46_{4,43}-45_{4,42}$ & 203653.642 & 6 & 236 & 590.6 & 161 & Blend with $\mathrm{C}_{2} \mathrm{H}_{5} \mathrm{CN}, \mathrm{C}_{2} \mathrm{H}_{5} \mathrm{OH}$, and $U$-line \\
\hline 341 & $46_{21,25}-45_{21,24}{ }^{\star}$ & 203679.015 & 17 & 672 & 471.1 & 161 & Blend with $\mathrm{CH}_{3} \mathrm{CN}, v_{8}=2$ \\
\hline 343 & $46_{23,23}-45_{23,22}$ 太 & 203766.086 & 29 & 762 & 446.4 & 364 & Blend with $\mathrm{C}_{2} \mathrm{H}_{5} \mathrm{OCHO}$ \\
\hline 345 & $47_{1,47}-46_{1,46}$ & 203773.957 & 14 & 227 & 607.7 & 364 & Blend with ${ }^{13} \mathrm{CH}_{2} \mathrm{CHCN}$ \\
\hline 346 & $46_{4,42}-45_{4,41}$ & 203841.799 & 6 & 236 & 590.6 & 364 & Blend with $\mathrm{C}_{2} \mathrm{H}_{5} \mathrm{CN}$ and $\mathrm{CH}_{3} \mathrm{OCHO}$ \\
\hline 347 & $47_{0,47}-46_{0,46}$ & 203894.538 & 14 & 227 & 607.7 & 364 & Blend with $U$-line \\
\hline 348 & $46_{2,44}-45_{2,43}$ & 205486.337 & 8 & 226 & 594.0 & 100 & Blend with $\mathrm{CH}_{3} \mathrm{OCHO}$ \\
\hline 349 & $41_{2,40}-40_{1,39}$ & 205486.671 & 20 & 176 & 17.7 & 100 & Blend with $\mathrm{CH}_{3} \mathrm{OCHO}$ \\
\hline 350 & $47_{2,46}-46_{2,45}$ & 206403.502 & 7 & 233 & 606.8 & 106 & Strong $\mathrm{C}_{2} \mathrm{H}_{5} \mathrm{CN}$ \\
\hline 351 & $47_{1,46}-46_{1,45}$ & 207565.934 & 6 & 232 & 607.1 & 282 & Blend with $\mathrm{C}_{2} \mathrm{H}_{5} \mathrm{OH}$ and $U$-line? \\
\hline 352 & $47_{9,38}-469,37^{\star}$ & 207811.666 & 7 & 313 & 585.8 & 173 & Blend with ${ }^{13} \mathrm{CH}_{3} \mathrm{OH}$ and $\mathrm{C}_{2} \mathrm{H}_{5} \mathrm{CN}$ \\
\hline 354 & $47_{10,37}-46_{10,36}$ * & 207811.909 & 8 & 332 & 580.5 & 173 & Blend with ${ }^{13} \mathrm{CH}_{3} \mathrm{OH}$ and $\mathrm{C}_{2} \mathrm{H}_{5} \mathrm{CN}$ \\
\hline 356 & $47_{11,36}-46_{11,35} \star$ & 207819.239 & 8 & 354 & 574.7 & 173 & Blend with $\mathrm{C}_{2} \mathrm{H}_{5} \mathrm{CN}$ and $\mathrm{C}_{2} \mathrm{H}_{5} \mathrm{OCHO}$ \\
\hline 358 & $47_{3,45}-46_{3,44}$ & 207820.872 & 6 & 239 & 605.5 & 173 & Blend with $\mathrm{C}_{2} \mathrm{H}_{5} \mathrm{CN}$ and $\mathrm{C}_{2} \mathrm{H}_{5} \mathrm{OCHO}$ \\
\hline 359 & $47_{8,40}-46_{8,39}{ }^{\star}$ & 207821.249 & 7 & 295 & 590.5 & 173 & Blend with $\mathrm{C}_{2} \mathrm{H}_{5} \mathrm{CN}, \mathrm{C}_{2} \mathrm{H}_{5} \mathrm{OCHO}$, and $\mathrm{C}_{2} \mathrm{H}_{5} \mathrm{OH}$ \\
\hline 361 & $47_{12,35}-46_{12,34}{ }^{\star}$ & 207832.034 & 9 & 378 & 568.5 & 173 & Strong NS, $\mathrm{CH}_{2}{ }^{13} \mathrm{CHCN}$, and $\mathrm{C}_{2} \mathrm{H}_{5} \mathrm{CN}$ \\
\hline 363 & $47_{7,41}-46_{7,40}^{\star}$ & 207845.603 & 7 & 280 & 594.7 & 173 & Strong $\mathrm{C}_{2} \mathrm{H}_{5} \mathrm{CN}, v_{13}=1 / v_{21}=1$ \\
\hline 365 & $47_{13,34}-46_{13,33^{\star}}$ & 207849.289 & 9 & 403 & 561.6 & 173 & Strong $\mathrm{C}_{2} \mathrm{H}_{5} \mathrm{CN}, v_{13}=1 / v_{21}=1$ \\
\hline 367 & $47_{14,33}-46_{14,32}{ }^{\star}$ & 207870.351 & 9 & 431 & 554.2 & 173 & Blend with $\mathrm{C}_{2} \mathrm{H}_{5} \mathrm{OH}$, uncertain baseline \\
\hline 369 & $47_{6,42}-46_{6,41}$ & 207894.282 & 6 & 267 & 598.2 & 173 & Blend with $\mathrm{C}_{2} \mathrm{H}_{5} \mathrm{OH}$ and $\mathrm{CH}_{2}{ }^{13} \mathrm{CHCN}$ \\
\hline 370 & $47_{6,41}-46_{6,40} \star$ & 207894.750 & 6 & 267 & 598.2 & 173 & Blend with $\mathrm{C}_{2} \mathrm{H}_{5} \mathrm{OH}$ and $\mathrm{CH}_{2}{ }^{13} \mathrm{CHCN}$ \\
\hline 372 & $47_{15,33}-46_{15,32}$ & 207894.786 & 10 & 461 & 546.2 & 173 & Blend with $\mathrm{C}_{2} \mathrm{H}_{5} \mathrm{OH}$ and $\mathrm{CH}_{2}{ }^{13} \mathrm{CHCN}$ \\
\hline 373 & $47_{16,31}-46_{16,30}$ * & 207922.295 & 10 & 492 & 537.6 & 173 & Blend with $\mathrm{C}_{2} \mathrm{H}_{3} \mathrm{CN}, v_{15}=1, \mathrm{C}_{2} \mathrm{H}_{5}{ }^{13} \mathrm{CN}$, and $\mathrm{CH}_{3} \mathrm{CH}_{3} \mathrm{CO}, v_{t}=1$ \\
\hline 375 & $47_{17,30}-46_{17,29}^{\star}$ & 207952.671 & 10 & 526 & 528.5 & 173 & Blend with $\mathrm{C}_{2} \mathrm{H}_{5} \mathrm{CN}$ and $\mathrm{CH}_{2}{ }^{13} \mathrm{CHCN}$ \\
\hline
\end{tabular}


Table 9. continued.

\begin{tabular}{|c|c|c|c|c|c|c|c|}
\hline$N^{a}$ & $\begin{array}{c}\text { Transition }^{b} \\
\text { (2) }\end{array}$ & $\begin{array}{c}\text { Frequency } \\
(\mathrm{MHz}) \\
\text { (3) }\end{array}$ & $\begin{array}{l}\text { Unc. }^{c} \\
(\mathrm{kHz}) \\
(4)\end{array}$ & $\begin{array}{l}E_{1}^{d} \\
(\mathrm{~K}) \\
(5)\end{array}$ & $\begin{array}{l}S \mu^{2} \\
\left(\mathrm{D}^{2}\right) \\
(6)\end{array}$ & $\begin{array}{c}\sigma^{e} \\
(\mathrm{mK}) \\
(7)\end{array}$ & Comments \\
\hline 377 & $15_{7,8}-16_{6,11}{ }^{\star}$ & 207955.703 & 25 & 66 & 1.4 & 173 & Blend with $\mathrm{C}_{2} \mathrm{H}_{5} \mathrm{CN}$ and $\mathrm{CH}_{2}{ }^{13} \mathrm{CHCN}$ \\
\hline 379 & $47_{5,43}-46_{5,42}$ & 207983.470 & 6 & 255 & 601.2 & 173 & Blend with $\mathrm{C}_{2} \mathrm{H}_{5} \mathrm{OH}$ and $\mathrm{CH}_{2}{ }^{13} \mathrm{CHCN}$ \\
\hline 380 & $47_{18,29}-46_{18,28}^{\star}$ & 207985.767 & 10 & 562 & 518.9 & 173 & Blend with $\mathrm{C}_{2} \mathrm{H}_{5} \mathrm{OH}$ and $\mathrm{CH}_{2}{ }^{13} \mathrm{CHCN}$ \\
\hline 382 & $47_{5,42}-46_{5,41}$ & 207996.582 & 6 & 255 & 601.2 & 173 & Blend with $\mathrm{C}_{2} \mathrm{H}_{5} \mathrm{CN}, \mathrm{C}_{2} \mathrm{H}_{5} \mathrm{OH}$, and $\mathrm{CH}_{2} \mathrm{CH}^{13} \mathrm{CN}$ \\
\hline 383 & $47_{19,28}-46_{19,27}{ }^{\star}$ & 208021.478 & 11 & 600 & 508.7 & 173 & Blend with $\mathrm{CH}_{2}{ }^{13} \mathrm{CHCN}$ \\
\hline 385 & $47_{20,27}-46_{20,26}{ }^{\star}$ & 208059.729 & 14 & 640 & 498.0 & 173 & Blend with $\mathrm{C}_{2} \mathrm{H}_{5} \mathrm{CN}$ \\
\hline 387 & $48_{1,48}-47_{1,47}$ & 208076.611 & 16 & 236 & 620.6 & 173 & Blend with $\mathrm{C}_{2} \mathrm{H}_{5} \mathrm{CN}$ and $\mathrm{CH}_{3} \mathrm{CH}_{3} \mathrm{CO}$ \\
\hline 388 & $47_{4,44}-46_{4,43}$ & 208085.930 & 6 & 246 & 603.7 & 173 & Blend with $\mathrm{CH}_{3} \mathrm{CH}_{3} \mathrm{CO}$ and $\mathrm{CH}_{2} \mathrm{CH}^{13} \mathrm{CN}$ \\
\hline 389 & $47_{21,26}-46_{21,25}{ }^{\star}$ & 208100.469 & 17 & 682 & 486.7 & 173 & $\begin{array}{l}\text { Blend with } \mathrm{CH}_{2} \mathrm{CH}^{13} \mathrm{CN}_{2} \mathrm{C}_{2} \mathrm{H}_{5} \mathrm{CN}, v_{13}=1 / v_{21}=1 \text {, } \\
\text { and } \mathrm{CH}_{2}{ }^{13} \mathrm{CHCN}\end{array}$ \\
\hline 391 & $48_{0,48}-47_{0,47}$ & 208185.442 & 16 & 236 & 620.6 & 173 & Blend with $\mathrm{C}_{2} \mathrm{H}_{5} \mathrm{OH}$ and $\mathrm{CH}_{2}{ }^{13} \mathrm{CHCN}$ \\
\hline 392 & $47_{23,24}-46_{23,23}{ }^{\star}$ & 208189.277 & 29 & 771 & 462.5 & 173 & Blend with $\mathrm{C}_{2} \mathrm{H}_{5} \mathrm{OH}$ and $\mathrm{CH}_{2}{ }^{13} \mathrm{CHCN}$ \\
\hline 394 & $47_{25,22}-46_{25,21}{ }^{\star}$ & 208287.742 & 46 & 869 & 436.0 & 168 & Blend with $\mathrm{C}_{2} \mathrm{H}_{5} \mathrm{OH}$ \\
\hline 396 & $47_{4,43}-46_{4,42}$ & 208302.740 & 7 & 246 & 603.7 & 168 & Blend with $\mathrm{C}_{2} \mathrm{H}_{5} \mathrm{CN}$ \\
\hline 397 & $47_{3,44}-46_{3,43}$ & 209390.960 & 9 & 239 & 605.7 & 58 & Blend with $\mathrm{C}_{2} \mathrm{H}_{5} \mathrm{OH}$ and $\mathrm{C}_{2} \mathrm{H}_{3} \mathrm{CN}, v_{15}=1$ \\
\hline 398 & $47_{2,45}-46_{2,44}$ & 209918.318 & 9 & 235 & 607.0 & 45 & Strong ${ }^{13} \mathrm{CH}_{3} \mathrm{CH}_{2} \mathrm{CN}, \mathrm{C}_{2} \mathrm{H}_{5} \mathrm{OH}$, and $\mathrm{CH}_{3} \mathrm{OCHO}$ \\
\hline 399 & $48_{2,47}-47_{2,46}$ & 210741.480 & 50 & 243 & 619.6 & 37 & $\begin{array}{l}\text { Blend with } \mathrm{C}_{2} \mathrm{H}_{3} \mathrm{CN}, v_{11}=3 \text { and } \mathrm{C}_{2} \mathrm{H}_{5} \mathrm{CN}, v_{13}=1 / v_{21}=1 \text {, } \\
\text { uncertain baseline }\end{array}$ \\
\hline 400 & $48_{1,47}-47_{1,46}$ & 211856.190 & 50 & 242 & 620.1 & 47 & Blend with $U$-line \\
\hline 401 & $48_{3,46}-47_{3,45}$ & 212219.540 & 50 & 249 & 618.5 & 36 & Blend with $\mathrm{C}_{2} \mathrm{H}_{5} \mathrm{CN}, v_{20}=1$ \\
\hline 402 & $48_{10,38}-47_{10,37^{\star}}$ & 212229.670 & 50 & 342 & 594.1 & 36 & Strong $\mathrm{C}_{2} \mathrm{H}_{5} \mathrm{CN}, v_{20}=1$ and $\mathrm{NH}_{2} \mathrm{CHO}$ \\
\hline 404 & $48_{9,39}-47_{9,38^{\star}}$ & 212230.190 & 50 & 323 & 599.2 & 36 & Strong $\mathrm{C}_{2} \mathrm{H}_{5} \mathrm{CN}, v_{20}=1$ and $\mathrm{NH}_{2} \mathrm{CHO}$ \\
\hline 406 & $48_{11,37}-47_{11,36^{\star}}$ & 212236.507 & 9 & 364 & 588.4 & 36 & Strong $\mathrm{C}_{2} \mathrm{H}_{5} \mathrm{CN}, v_{20}=1$ and $\mathrm{NH}_{2} \mathrm{CHO}$ \\
\hline 408 & $48_{8,40}-47_{8,39^{\star}}$ & 212241.200 & 50 & 305 & 603.8 & 36 & Strong $\mathrm{NH}_{2} \mathrm{CHO}$ \\
\hline 410 & $48_{12,36}-47_{12,35}{ }^{\star}$ & 212249.060 & 50 & 388 & 582.2 & 36 & Strong $\mathrm{NH}_{2} \mathrm{CHO}$ and $\mathrm{NH}_{2} \mathrm{CHO}, v_{12}=1$ \\
\hline 412 & $48_{13,35}-47_{13,34}{ }^{\star}$ & 212266.320 & 50 & 413 & 575.5 & 36 & Strong $\mathrm{C}_{2} \mathrm{H}_{5} \mathrm{OH}, \mathrm{NH}_{2}{ }^{13} \mathrm{CHO}$, and $\mathrm{NH}_{2} \mathrm{CHO}$ \\
\hline 414 & $48_{7,41}-47_{7,40}{ }^{\star}$ & 212267.790 & 50 & 290 & 607.8 & 36 & Strong $\mathrm{C}_{2} \mathrm{H}_{5} \mathrm{OH}, \mathrm{NH}_{2}{ }^{13} \mathrm{CHO}$, and $\mathrm{NH}_{2} \mathrm{CHO}$ \\
\hline 416 & $48_{14,34}-47_{14,33}{ }^{\star}$ & 212287.590 & 50 & 441 & 568.2 & 36 & Blend with $\mathrm{C}_{2} \mathrm{H}_{5} \mathrm{CN}, v_{13}=1 / v_{21}=1$ and $v=0$, and $U$-line \\
\hline 418 & $48_{15,33}-47_{15,32^{\star}}$ & 212312.290 & 50 & 471 & 560.4 & 36 & Strong $\mathrm{C}_{2} \mathrm{H}_{3} \mathrm{CN}, v_{11}=1$ and $\mathrm{NH}_{2} \mathrm{CHO}$ \\
\hline 420 & $48_{6,43}-47_{6,42}$ & 212320.280 & 50 & 276 & 611.3 & 36 & Strong $\mathrm{C}_{2} \mathrm{H}_{3} \mathrm{CN}, v_{11}=1$ and $\mathrm{NH}_{2} \mathrm{CHO}$ \\
\hline 421 & $48_{6,42}-47_{6,41}$ & 212320.770 & 50 & 276 & 611.3 & 36 & Strong $\mathrm{C}_{2} \mathrm{H}_{3} \mathrm{CN}, v_{11}=1$ and $\mathrm{NH}_{2} \mathrm{CHO}$ \\
\hline 422 & $48_{16,32}-47_{16,31}{ }^{\star}$ & 212340.190 & 50 & 502 & 552.0 & 36 & Strong $\mathrm{C}_{2} \mathrm{H}_{3} \mathrm{CN}$ \\
\hline 424 & $48_{17,31}-47_{17,30}{ }^{\star}$ & 212371.060 & 50 & 536 & 543.1 & 36 & Blend with $\mathrm{NH}_{2}{ }^{13} \mathrm{CHO}, v_{12}=1$, uncertain baseline \\
\hline 426 & $14_{7,7}-15_{6,10^{\star}}$ & 212375.891 & 25 & 63 & 1.2 & 36 & Blend with $\mathrm{NH}_{2}{ }^{13} \mathrm{CHO}, v_{12}=1$, uncertain baseline \\
\hline 428 & $49_{1,49}-48_{1,48}$ & 212378.480 & 50 & 246 & 633.5 & 36 & Blend with $\mathrm{NH}_{2}{ }^{13} \mathrm{CHO}, v_{12}=1$, uncertain baseline \\
\hline 429 & $48_{18,30}-47_{18,29}{ }^{\star}$ & 212404.720 & 50 & 572 & 533.7 & 36 & Strong $\mathrm{NH}_{2} \mathrm{CHO}, v_{12}=1$ and $\mathrm{H}^{13} \mathrm{CCCN}, v_{7}=1$ \\
\hline 431 & $48_{5,44}-47_{5,43}$ & 212415.060 & 50 & 265 & 614.2 & 36 & Blend with $\mathrm{NH}_{2} \mathrm{CHO}$ \\
\hline 432 & $48_{5,43}-47_{5,42}$ & 212430.890 & 50 & 265 & 614.3 & 36 & Strong $\mathrm{NH}_{2} \mathrm{CHO}$ \\
\hline 433 & $48_{19,29}-47_{19,28}{ }^{\star}$ & 212441.110 & 50 & 610 & 523.7 & 99 & Blend with $\mathrm{NH}_{2} \mathrm{CHO}$ and $\mathrm{CH}_{3} \mathrm{OCHO}$ \\
\hline 435 & $49_{0,49}-48_{0,48}$ & 212476.620 & 50 & 246 & 633.6 & 99 & Blend with $\mathrm{C}_{2} \mathrm{H}_{5} \mathrm{OH}$ and $\mathrm{CH}_{3} \mathrm{OCHO}$ \\
\hline 436 & $48_{20,28}-47_{20,27}{ }^{\star}$ & 212480.030 & 50 & 650 & 513.3 & 99 & Blend with ${ }^{13} \mathrm{CH}_{3} \mathrm{OH}, v_{t}=1$ and $\mathrm{CH}_{3} \mathrm{CHO}$ \\
\hline 438 & $48_{4,45}-47_{4,44}$ & 212517.790 & 50 & 256 & 616.8 & 99 & Blend with $\mathrm{C}_{2} \mathrm{H}_{5} \mathrm{CN}$ and $\mathrm{C}_{2} \mathrm{H}_{5} \mathrm{OH}$, uncertain baseline \\
\hline 439 & $48_{21,27}-47_{21,26}{ }^{\star}$ & 212521.490 & 50 & 692 & 502.1 & 99 & Blend with $\mathrm{C}_{2} \mathrm{H}_{5} \mathrm{CN}$ and $\mathrm{C}_{2} \mathrm{H}_{5} \mathrm{OH}$, uncertain baseline \\
\hline 441 & $48_{26,22}-47_{26,21} \star$ & 212766.374 & 57 & 931 & 438.8 & 99 & Blend with ${ }^{13} \mathrm{CH}_{3} \mathrm{OH}$ and $\mathrm{C}_{2} \mathrm{H}_{5} \mathrm{CN}$, uncertain baseline \\
\hline 443 & $48_{4,44}-47_{4,43}$ & 212766.640 & 50 & 256 & 616.7 & 99 & Blend with ${ }^{13} \mathrm{CH}_{3} \mathrm{OH}$ and $\mathrm{C}_{2} \mathrm{H}_{5} \mathrm{CN}$, uncertain baseline \\
\hline 444 & $48_{3,45}-47_{3,44}$ & 213912.029 & 10 & 249 & 618.6 & 48 & Blend with ${ }^{13} \mathrm{CH}_{3} \mathrm{OH}$ \\
\hline 445 & $48_{2,46}-47_{2,45}$ & 214343.680 & 50 & 245 & 619.9 & 75 & Strong ${ }^{13} \mathrm{CH}_{3} \mathrm{CN}$ and $\mathrm{C}_{2} \mathrm{H}_{5}{ }^{13} \mathrm{CN}$ \\
\hline 446 & $49_{2,48}-48_{2,47}$ & 215077.170 & 50 & 253 & 632.6 & 74 & Strong $\mathrm{C}_{2} \mathrm{H}_{5} \mathrm{CN}$ \\
\hline 447 & $49_{1,48}-48_{1,47}$ & 216142.273 & 8 & 252 & 632.9 & 55 & Blend with OCS and $U$-line \\
\hline 448 & $49_{3,47}-48_{3,46}$ & 216615.615 & 6 & 259 & 631.5 & 55 & Blend with $\mathrm{CH}_{3} \mathrm{COOH}$ and $\mathrm{CH}_{3} \mathrm{CHO}$ \\
\hline 449 & $49_{10,39}-48_{10,38^{\star}}$ & 216647.130 & 8 & 352 & 607.5 & 55 & Strong $\mathrm{SO}_{2}$ and $\mathrm{C}_{2} \mathrm{H}_{5} \mathrm{OH}$ \\
\hline 451 & $49_{9,40}-489,39^{\star}$ & 216648.604 & 8 & 333 & 612.6 & 55 & Strong $\mathrm{SO}_{2}$ and $\mathrm{C}_{2} \mathrm{H}_{5} \mathrm{OH}$ \\
\hline 453 & $49_{11,38}-48_{11,37^{\star}}$ & 216653.496 & 9 & 374 & 602.0 & 55 & Strong $\mathrm{SO}_{2}$ and $\mathrm{C}_{2} \mathrm{H}_{5} \mathrm{OH}$ \\
\hline 455 & $49_{8,42}-48_{8,41} \star \star$ & 216661.026 & 7 & 315 & 617.0 & 55 & Strong $\mathrm{SO}_{2}$ and $\mathrm{C}_{2} \mathrm{H}_{5} \mathrm{OH}$ \\
\hline 457 & $49_{12,37}-48_{12,36}{ }^{\star}$ & 216665.865 & 9 & 398 & 596.0 & 55 & Strong $\mathrm{SO}_{2}$ and $\mathrm{C}_{2} \mathrm{H}_{5} \mathrm{OH}$ \\
\hline 459 & $50_{1,50}-49_{1,49}$ & 216679.385 & 20 & 257 & 646.5 & 55 & Blend with $\mathrm{H}_{2} \mathrm{~S}$ \\
\hline 460 & $49_{13,36}-48_{13,35}{ }^{\star}$ & 216683.096 & 10 & 423 & 589.3 & 55 & Blend with $\mathrm{H}_{2} \mathrm{~S}$ \\
\hline 462 & $49_{7,43}-48_{7,42^{\star}}^{\star}$ & 216690.012 & 7 & 300 & 621.0 & 55 & Strong $\mathrm{H}_{2} \mathrm{~S}$ \\
\hline 464 & $49_{14,35}-48_{14,34}{ }^{\star}$ & 216704.448 & 10 & 451 & 582.2 & 55 & Strong $\mathrm{H}_{2} \mathrm{~S}$ \\
\hline 466 & $49_{15,34}-48_{15,33^{\star}}$ & 216729.429 & 10 & 481 & 574.6 & 50 & Strong $\mathrm{H}_{2} \mathrm{~S}$ and $\mathrm{C}_{2} \mathrm{H}_{5} \mathrm{CN}, v_{13}=1 / v_{21}=1$ \\
\hline 468 & $49_{6,44}-48_{6,43}$ & 216746.358 & 7 & 287 & 624.5 & 50 & Strong $\mathrm{C}_{2} \mathrm{H}_{5} \mathrm{CN}$ \\
\hline 469 & $49_{6,43}-48_{6,42}$ & 216747.094 & 7 & 287 & 624.5 & 50 & Strong $\mathrm{C}_{2} \mathrm{H}_{5} \mathrm{CN}$ \\
\hline 470 & $49_{16,33}-48_{16,32}$ * & 216757.710 & 50 & 513 & 566.3 & 50 & Strong $\mathrm{C}_{2} \mathrm{H}_{5} \mathrm{CN}$ \\
\hline 472 & $50_{0,50}-49_{0,49}$ & 216767.700 & 50 & 256 & 646.5 & 50 & Blend with $\mathrm{C}_{2} \mathrm{H}_{5} \mathrm{CN}$ and $U$-line \\
\hline
\end{tabular}


Table 9. continued.

\begin{tabular}{|c|c|c|c|c|c|c|c|}
\hline$N^{a}$ & Transition $^{b}$ & $\begin{array}{c}\text { Frequency } \\
(\mathrm{MHz}) \\
(3)\end{array}$ & $\begin{array}{c}\text { Unc. }^{c} \\
(\mathrm{kHz}) \\
(4)\end{array}$ & $\begin{array}{l}E_{1}^{d} \\
(\mathrm{~K}) \\
(5)\end{array}$ & $\begin{array}{l}S \mu^{2} \\
\left(\mathrm{D}^{2}\right) \\
(6)\end{array}$ & $\begin{array}{c}\sigma^{e} \\
(\mathrm{mK}) \\
(7)\end{array}$ & Comments \\
\hline 473 & $49_{17,32}-48_{17,31}$ * & 216789.070 & 50 & 546 & 557.7 & 50 & Blend with $\mathrm{CH}_{3} \mathrm{CH}_{3} \mathrm{CO}, v_{t}=1$ \\
\hline 475 & $13_{7,6}-14_{6,9}{ }^{\star}$ & 216795.931 & 26 & 59 & 1.0 & 50 & Blend with $\mathrm{CH}_{3} \mathrm{CH}_{3} \mathrm{CO}, v_{t}=1$ \\
\hline 477 & $49_{18,31}-48_{18,30}$ * & 216823.235 & 10 & 582 & 548.3 & 50 & Strong $\mathrm{CH}_{3} \mathrm{OCHO}$ \\
\hline 479 & $49_{5,45}-48_{5,44}$ & 216847.000 & 7 & 275 & 627.3 & 50 & Strong $\mathrm{C}_{2} \mathrm{H}_{5} \mathrm{CN}, v_{13}=1 / v_{21}=1$ \\
\hline 480 & $49_{19,30}-48_{19,29}{ }^{\star}$ & 216860.211 & 11 & 620 & 538.7 & 50 & Blend with $\mathrm{C}_{2} \mathrm{H}_{5} \mathrm{CN}, v_{13}=1 / v_{21}=1$ and $U$-line \\
\hline 482 & $49_{5,44}-48_{5,43}$ & 216865.924 & 7 & 275 & 627.4 & 50 & Blend with $\mathrm{C}_{2} \mathrm{H}_{5} \mathrm{CN}, v_{13}=1 / v_{21}=1$ and $U$-line \\
\hline 483 & $49_{20,29}-48_{20,28}{ }^{\star}$ & 216899.870 & 50 & 660 & 528.3 & 50 & Blend with $U$-line \\
\hline 485 & $49_{21,28}-48_{21,27}$ * & 216942.142 & 17 & 702 & 517.5 & 50 & Strong $\mathrm{CH}_{3} \mathrm{OH}$ \\
\hline 487 & $49_{4,46}-48_{4,45}$ & 216949.090 & 50 & 266 & 629.7 & 50 & Strong $\mathrm{CH}_{3} \mathrm{OH}$ \\
\hline 488 & $42_{10,32}-43_{9,35}$ * & 216956.684 & 77 & 284 & 6.3 & 50 & Strong $\mathrm{CH}_{3} \mathrm{OH}$ \\
\hline 490 & $49_{4,45}-48_{4,44}$ & 217233.695 & 7 & 266 & 629.8 & 50 & Strong $\mathrm{CH}_{2}{ }^{13} \mathrm{CHCN}$ \\
\hline 491 & $45_{2,44}-44_{1,43}$ & 217511.675 & 25 & 213 & 21.1 & 50 & Blend with ${ }^{13} \mathrm{CN}$ in absorption and $U$-line \\
\hline 492 & $50_{1,50}-49_{0,49}$ & 217512.520 & 22 & 256 & 41.1 & 50 & Blend with ${ }^{13} \mathrm{CN}$ in absorption and $U$-line \\
\hline 493 & $50_{2,49}-49_{2,48}$ & 219410.228 & 10 & 263 & 645.7 & 92 & Strong $\mathrm{HC}_{3} \mathrm{~N}, v_{6}=v_{7}=1$ \\
\hline 494 & $6_{5,2}-5_{4,1}{ }^{\star}$ & 219563.799 & 11 & 20 & 4.4 & 92 & Strong $\mathrm{C}^{18} \mathrm{O}$ \\
\hline 496 & $50_{1,49}-49_{1,48}$ & 220424.730 & 8 & 262 & 645.9 & 98 & Strong $\mathrm{CH}_{3}{ }^{13} \mathrm{CN}$ \\
\hline 497 & $51_{4,47}-50_{4,46}$ & 226177.852 & 9 & 287 & 655.8 & 278 & Noisy \\
\hline 498 & $51_{3,48}-50_{3,47}$ & 227473.842 & 14 & 281 & 657.7 & 85 & Blend with $\mathrm{CH}_{2} \mathrm{CH}^{13} \mathrm{CN}_{\text {and }} \mathrm{C}_{2} \mathrm{H}_{5} \mathrm{OCHO}$, uncertain baseline \\
\hline 499 & $51_{2,49}-50_{2,48}$ & 227577.811 & 11 & 277 & 658.7 & 85 & Strong $\mathrm{CH}_{3} \mathrm{OCHO}$ and $\mathrm{C}_{2} \mathrm{H}_{5} \mathrm{OH}$ \\
\hline 500 & $29_{3,27}-28_{2,26}$ & 227580.141 & 9 & 91 & 7.6 & 85 & Strong $\mathrm{CH}_{3} \mathrm{OCHO}$ and $\mathrm{C}_{2} \mathrm{H}_{5} \mathrm{OH}$ \\
\hline 501 & $53_{2,52}-52_{2,51}$ & 232395.004 & 14 & 295 & 684.5 & 19 & Blend with $\mathrm{C}_{2} \mathrm{H}_{5} \mathrm{OH}$ \\
\hline 502 & $95,5^{-8}-844^{\star}$ & 232831.850 & 11 & 24 & 4.9 & 19 & Blend with $\mathrm{CH}_{3} \mathrm{CH}_{3} \mathrm{CO}, v_{t}=1$ and $U$-line \\
\hline 504 & $62_{6,57}-62_{5,58}$ & 232836.738 & 28 & 440 & 32.6 & 19 & Blend with $\mathrm{CH}_{3} \mathrm{CH}_{3} \mathrm{CO}, v_{t}=1$ and $U$-line \\
\hline 505 & $44_{6,39}-44_{5,40}$ & 235131.269 & 9 & 236 & 22.2 & 131 & Strong $\mathrm{C}_{2} \mathrm{H}_{5} \mathrm{OH}$ \\
\hline 506 & $53_{4,49}-524,48$ & 235136.432 & 11 & 309 & 681.8 & 131 & Strong $\mathrm{C}_{2} \mathrm{H}_{5} \mathrm{OH}$ \\
\hline 507 & $39_{6,33}-39_{5,34}$ & 235437.401 & 9 & 191 & 19.4 & 131 & Blend with ${ }^{13} \mathrm{CH}_{3} \mathrm{OH}, v_{t}=1$ \\
\hline 508 & $38_{6,32}-38_{5,33}$ & 235489.643 & 9 & 183 & 18.9 & 131 & Blend with $\mathrm{CH}_{3} \mathrm{CH}_{3} \mathrm{CO}$ and $\mathrm{HC}^{13} \mathrm{CCN}$ \\
\hline 509 & $37_{6,31}-37_{5,32}$ & 235537.303 & 9 & 175 & 18.4 & 131 & Strong $\mathrm{HCC}^{13} \mathrm{CN}$ \\
\hline 510 & $37_{6,32}-37_{5,33}$ & 235543.778 & 9 & 175 & 18.4 & 131 & Strong $\mathrm{HCC}^{13} \mathrm{CN}$ and $\mathrm{CH}_{3} \mathrm{CH}_{3} \mathrm{CO}$ \\
\hline 511 & $36_{6,30}-36_{5,31}$ & 235580.671 & 9 & 167 & 17.8 & 131 & Blend with $\mathrm{C}_{2} \mathrm{H}_{3} \mathrm{CN}$, uncertain baseline \\
\hline 512 & $36_{6,31}-36_{5,32}$ & 235585.618 & 9 & 167 & 17.8 & 131 & Blend with $\mathrm{C}_{2} \mathrm{H}_{3} \mathrm{CN}$, uncertain baseline \\
\hline 513 & $35_{6,29}-35_{5,30}$ & 235620.022 & 9 & 159 & 17.3 & 131 & Blend with $\mathrm{C}_{2} \mathrm{H}_{5} \mathrm{CN}, v_{13}=1 / v_{21}=1$ \\
\hline 514 & $35_{6,30}-35_{5,31}$ & 235623.773 & 9 & 159 & 17.3 & 131 & Blend with $\mathrm{C}_{2} \mathrm{H}_{5} \mathrm{CN}, v_{13}=1 / v_{21}=1$ \\
\hline 515 & $34_{6,28}-34_{5,29}$ & 235655.616 & 9 & 152 & 16.7 & 131 & $\begin{array}{l}\text { Blend with } \mathrm{C}_{2} \mathrm{H}_{5} \mathrm{CN}, v_{13}=1 / v_{21}=1, \mathrm{HC}^{13} \mathrm{CCN}, v_{5}=1 / v_{7}=3 \text {, } \\
\text { and } \mathrm{C}_{2} \mathrm{H}_{3} \mathrm{CN}, v_{11}=1\end{array}$ \\
\hline 516 & $34_{6,29}-34_{5,30}$ & 235658.436 & 9 & 152 & 16.7 & 131 & $\begin{array}{l}\text { Blend with } \mathrm{C}_{2} \mathrm{H}_{5} \mathrm{CN}, v_{13}=1 / v_{21}=1, \mathrm{HC}^{13} \mathrm{CCN}, v_{5}=1 / v_{7}=3 \text {, } \\
\text { and } \mathrm{C}_{2} \mathrm{H}_{3} \mathrm{CN}, v_{11}=1\end{array}$ \\
\hline 517 & $33_{6,27}-33_{5,28}$ & 235687.700 & 10 & 145 & 16.2 & 131 & Blend with $\mathrm{HCC}^{13} \mathrm{CN}, v_{5}=1 / v_{7}=3$, uncertain baseline \\
\hline 518 & $33_{6,28}-33_{5,29}$ & 235689.803 & 10 & 145 & 16.2 & 131 & Blend with $\mathrm{HCC}^{13} \mathrm{CN}, v_{5}=1 / v_{7}=3$, uncertain baseline \\
\hline 519 & $32,26-325,27$ & 235716.510 & 10 & 138 & 15.7 & 131 & Blend with $\mathrm{CH}_{3} \mathrm{OCH}_{3}$ \\
\hline 520 & $32,27-32,5,28$ & 235718.063 & 10 & 138 & 15.7 & 131 & Blend with $\mathrm{CH}_{3} \mathrm{OCH}_{3}$ \\
\hline 521 & $31_{6,25}-31_{5,26}$ & 235742.268 & 10 & 131 & 15.1 & 131 & Blend with $U$-line in absorption or baseline problem? \\
\hline 522 & $31_{6,26}-31_{5,27}$ & 235743.404 & 10 & 131 & 15.1 & 131 & Blend with $U$-line in absorption or baseline problem? \\
\hline 523 & $30_{6,24}-30_{5,25}$ & 235765.188 & 10 & 124 & 14.6 & 131 & Blend with $U$-line in absorption or baseline problem? \\
\hline 524 & $30_{6,25}-30_{5,26}$ & 235766.010 & 10 & 124 & 14.6 & 131 & Blend with $U$-line in absorption or baseline problem? \\
\hline 525 & $29_{6,23}-29_{5,24}$ & 235785.473 & 10 & 118 & 14.1 & 131 & Blend with $\mathrm{CH}_{3} \mathrm{OCH}_{3}$ and ${ }^{13} \mathrm{CH}_{3} \mathrm{CH}_{2} \mathrm{CN}$ \\
\hline 526 & $29_{6,24}-29_{5,25}$ & 235786.062 & 10 & 118 & 14.1 & 131 & Blend with $\mathrm{CH}_{3} \mathrm{OCH}_{3}$ and ${ }^{13} \mathrm{CH}_{3} \mathrm{CH}_{2} \mathrm{CN}$ \\
\hline 527 & $28_{6,22}-28_{5,23}$ & 235803.318 & 11 & 112 & 13.6 & 131 & Blend with $\mathrm{CH}_{3} \mathrm{CH}_{3} \mathrm{CO}, v_{t}=1$ \\
\hline 528 & $28_{6,23}-28_{5,24}$ & 235803.734 & 11 & 112 & 13.6 & 131 & Blend with $\mathrm{CH}_{3} \mathrm{CH}_{3} \mathrm{CO}, v_{t}=1$ \\
\hline 529 & $27_{6,21}-27_{5,22}$ & 235818.908 & 11 & 106 & 13.0 & 131 & Blend with $\mathrm{CH}_{3} \mathrm{CHO}$, uncertain baseline \\
\hline 530 & $27_{6,22}-27_{5,23}$ & 235819.199 & 11 & 106 & 13.0 & 131 & Blend with $\mathrm{CH}_{3} \mathrm{CHO}$, uncertain baseline \\
\hline 531 & $26_{6,20}-26_{5,21}$ & 235832.420 & 11 & 100 & 12.5 & 131 & Blend with $\mathrm{CH}_{3} \mathrm{OCHO}$ \\
\hline 532 & $26_{6,21}-26_{5,22}$ & 235832.620 & 11 & 100 & 12.5 & 131 & Blend with $\mathrm{CH}_{3} \mathrm{OCHO}$ \\
\hline 533 & $25_{6,19}-25_{5,20}$ & 235844.025 & 11 & 95 & 12.0 & 131 & Strong $\mathrm{CH}_{3} \mathrm{OCHO}$ \\
\hline 534 & $25_{6,20}-25_{5,21}$ & 235844.161 & 11 & 95 & 12.0 & 131 & Strong $\mathrm{CH}_{3} \mathrm{OCHO}$ \\
\hline 535 & $24_{6,18}-24_{5,19}^{\star}$ & 235853.884 & 12 & 89 & 11.4 & 131 & Blend with $\mathrm{CH}_{3} \mathrm{OCHO}$ \\
\hline 537 & $23_{6,17}-23_{5,18}{ }^{\star}$ & 235862.152 & 12 & 84 & 10.9 & 131 & Strong $\mathrm{CH}_{3} \mathrm{OCHO}$ and $\mathrm{C}_{2} \mathrm{H}_{5} \mathrm{CN}, v_{13}=1 / v_{21}=1$ \\
\hline 539 & $22_{6,16}-22_{5,17^{\star}}$ & 235868.978 & 12 & 79 & 10.4 & 131 & Strong $\mathrm{CH}_{3} \mathrm{OCHO}$ and $\mathrm{C}_{2} \mathrm{H}_{5} \mathrm{CN}, v_{13}=1 / v_{21}=1$ \\
\hline 541 & $21_{6,15}-21_{5,16}{ }^{\star}$ & 235874.503 & 13 & 75 & 9.9 & 131 & Strong ${ }^{13} \mathrm{CH}_{3} \mathrm{OH}, \mathrm{CH}_{3} \mathrm{OCHO}$, and $\mathrm{HC}^{13} \mathrm{CCN}, v_{6}=1$ \\
\hline 543 & $6_{6,0}-6_{5,1}{ }^{\star}$ & 235877.922 & 15 & 30 & 0.9 & 131 & Strong ${ }^{13} \mathrm{CH}_{3} \mathrm{OH}, \mathrm{CH}_{3} \mathrm{OCHO}$, and $\mathrm{HC}^{13} \mathrm{CCN}, v_{6}=1$ \\
\hline 545 & $20_{6,14}-20_{5,15}{ }^{\star}$ & 235878.862 & 13 & 70 & 9.3 & 131 & Strong ${ }^{13} \mathrm{CH}_{3} \mathrm{OH}, \mathrm{CH}_{3} \mathrm{OCHO}$, and $\mathrm{HC}^{13} \mathrm{CCN}, v_{6}=1$ \\
\hline 547 & $7_{6,1}-7_{5,2} \star$ & 235879.246 & 15 & 32 & 1.7 & 131 & Strong ${ }^{13} \mathrm{CH}_{3} \mathrm{OH}, \mathrm{CH}_{3} \mathrm{OCHO}$, and $\mathrm{HC}^{13} \mathrm{CCN}, v_{6}=1$ \\
\hline 549 & $8_{6,2}-8_{5,3}{ }^{\star}$ & 235880.638 & 15 & 33 & 2.4 & 131 & Strong ${ }^{13} \mathrm{CH}_{3} \mathrm{OH}, \mathrm{CH}_{3} \mathrm{OCHO}$, and $\mathrm{HC}^{13} \mathrm{CCN}, v_{6}=1$ \\
\hline 551 & $9_{6,3}-9_{5,4}^{\star}$ & 235882.048 & 15 & 35 & 3.1 & 131 & Strong ${ }^{13} \mathrm{CH}_{3} \mathrm{OH}, \mathrm{CH}_{3} \mathrm{OCHO}$, and $\mathrm{HC}^{13} \mathrm{CCN}, v_{6}=1$ \\
\hline
\end{tabular}


Table 9. continued.

\begin{tabular}{|c|c|c|c|c|c|c|c|}
\hline$N^{a}$ & Transition $^{b}$ & $\begin{array}{c}\text { Frequency } \\
\text { (MHz) } \\
\text { (3) }\end{array}$ & $\begin{array}{l}\text { Unc. }^{c} \\
(\mathrm{kHz}) \\
(4)\end{array}$ & $\begin{array}{l}E_{1}{ }^{d} \\
(\mathrm{~K}) \\
(5)\end{array}$ & $\begin{array}{l}S \mu^{2} \\
\left(\mathrm{D}^{2}\right) \\
(6)\end{array}$ & $\begin{array}{c}\sigma^{e} \\
(\mathrm{mK}) \\
(7)\end{array}$ & Comments \\
\hline 553 & $19_{6,13}-19_{5,14^{\star}}$ & 235882.182 & 13 & 66 & 8.8 & 131 & Strong ${ }^{13} \mathrm{CH}_{3} \mathrm{OH}, \mathrm{CH}_{3} \mathrm{OCHO}$, and $\mathrm{HC}^{13} \mathrm{CCN}, v_{6}=1$ \\
\hline 555 & $10_{6,4}-10_{5,5}{ }^{\star}$ & 235883.423 & 15 & 37 & 3.7 & 131 & Strong ${ }^{13} \mathrm{CH}_{3} \mathrm{OH}, \mathrm{CH}_{3} \mathrm{OCHO}$, and $\mathrm{HC}^{13} \mathrm{CCN}, v_{6}=1$ \\
\hline 557 & $18_{6,12}-18_{5,13}^{\star}$ & 235884.587 & 13 & 62 & 8.2 & 131 & Strong ${ }^{13} \mathrm{CH}_{3} \mathrm{OH}, \mathrm{CH}_{3} \mathrm{OCHO}$, and $\mathrm{HC}^{13} \mathrm{CCN}, v_{6}=1$ \\
\hline 559 & $11_{6,5}-11_{5,6}{ }^{\star}$ & 235884.702 & 15 & 40 & 4.3 & 131 & Strong ${ }^{13} \mathrm{CH}_{3} \mathrm{OH}, \mathrm{CH}_{3} \mathrm{OCHO}$, and $\mathrm{HC}^{13} \mathrm{CCN}, v_{6}=1$ \\
\hline 561 & $12_{6,6}-125,7^{\star}$ & 235885.818 & 15 & 42 & 4.9 & 131 & Strong ${ }^{13} \mathrm{CH}_{3} \mathrm{OH}, \mathrm{CH}_{3} \mathrm{OCHO}$, and $\mathrm{HC}^{13} \mathrm{CCN}, v_{6}=1$ \\
\hline 563 & $17_{6,11}-17_{5,12^{\star}}$ & 235886.191 & 14 & 58 & 7.7 & 131 & Strong ${ }^{13} \mathrm{CH}_{3} \mathrm{OH}, \mathrm{CH}_{3} \mathrm{OCHO}$, and $\mathrm{HC}^{13} \mathrm{CCN}, v_{6}=1$ \\
\hline 565 & $13_{6,7}-13_{5,8}^{\star}$ & 235886.698 & 14 & 45 & 5.5 & 131 & Strong ${ }^{13} \mathrm{CH}_{3} \mathrm{OH}, \mathrm{CH}_{3} \mathrm{OCHO}$, and $\mathrm{HC}^{13} \mathrm{CCN}, v_{6}=1$ \\
\hline 567 & $16_{6,10}-16_{5,11}^{\star}$ & 235887.104 & 14 & 55 & 7.2 & 131 & Strong ${ }^{13} \mathrm{CH}_{3} \mathrm{OH}, \mathrm{CH}_{3} \mathrm{OCHO}$, and $\mathrm{HC}^{13} \mathrm{CCN}, v_{6}=1$ \\
\hline 569 & $14_{6,8}-14_{5,9^{\star}}^{\star}$ & 235887.263 & 14 & 48 & 6.1 & 131 & Strong ${ }^{13} \mathrm{CH}_{3} \mathrm{OH}, \mathrm{CH}_{3} \mathrm{OCHO}$, and $\mathrm{HC}^{13} \mathrm{CCN}, v_{6}=1$ \\
\hline 571 & $15_{6,9}-15_{5,10^{\star}}$ 太 & 235887.429 & 14 & 51 & 6.6 & 131 & Strong ${ }^{13} \mathrm{CH}_{3} \mathrm{OH}, \mathrm{CH}_{3} \mathrm{OCHO}$, and $\mathrm{HC}^{13} \mathrm{CCN}, v_{6}=1$ \\
\hline 573 & $53_{2,51}-52_{2,50}$ & 236363.740 & 13 & 299 & 684.6 & 37 & Strong $\mathrm{CH}_{2} \mathrm{CH}^{13} \mathrm{CN}$ \\
\hline 574 & $53_{3,50}-52_{3,49}$ & 236507.754 & 18 & 303 & 683.6 & 37 & Strong $\mathrm{HC}_{3} \mathrm{~N}$ \\
\hline 575 & $54_{3,51}-53_{3,50}$ & 241020.744 & 20 & 314 & 696.6 & 216 & Strong $\mathrm{C}^{34} \mathrm{~S}$ \\
\hline 576 & $55_{2,54}-54_{2,53}$ & 241040.003 & 19 & 318 & 710.3 & 216 & Strong $\mathrm{C}_{2} \mathrm{H}_{3} \mathrm{CN}, v_{11}=2$ and $\mathrm{CH}_{3} \mathrm{OH}$ \\
\hline 577 & $55_{4,51}-54_{4,50}$ & 244110.418 & 14 & 332 & 707.8 & 46 & Blend with $\mathrm{CH}_{3} \mathrm{CH}_{3} \mathrm{CO}, v_{t}=1, \mathrm{C}_{2} \mathrm{H}_{3} \mathrm{CN}, v_{11}=1$, and $U$-line \\
\hline 578 & $55_{2,53}-54_{2,52}$ & 245118.648 & 14 & 322 & 710.4 & 72 & Blend with $\mathrm{CH}_{2} \mathrm{NH}$ \\
\hline 579 & $56_{2,55}-55_{2,54}$ & 245359.219 & 21 & 329 & 723.3 & 72 & Strong ${ }^{13} \mathrm{CH}_{3} \mathrm{CH}_{2} \mathrm{CN}$ \\
\hline 580 & $55_{3,52}-54_{3,51}$ & 245530.359 & 22 & 326 & 709.6 & 72 & Strong $\mathrm{HCC}^{13} \mathrm{CN}, v_{7}=1$ and ${ }^{13} \mathrm{CH}_{3} \mathrm{CH}_{2} \mathrm{CN}$ \\
\hline 581 & $58_{1,57}-57_{2,56}$ & 247309.555 & 23 & 353 & 33.7 & 68 & Blend with $\mathrm{C}_{2} \mathrm{H}_{3} \mathrm{CN}, v_{15}=1$ \\
\hline 582 & $56_{3,54}-55_{3,53}$ & 247311.322 & 10 & 336 & 722.3 & 68 & Blend with $\mathrm{C}_{2} \mathrm{H}_{3} \mathrm{CN}, v_{15}=1$ \\
\hline 583 & $57_{10,47}-56_{10,46^{\star}}$ & 251977.746 & 9 & 442 & 714.8 & 42 & Strong $\mathrm{CH}_{3} \mathrm{OH}$ \\
\hline 585 & $57_{11,46}-56_{11,45^{\star}}$ & 251978.622 & 9 & 463 & 709.9 & 42 & Strong $\mathrm{CH}_{3} \mathrm{OH}$ \\
\hline 587 & $57_{12,45^{-}}-56_{12,44^{\star}}$ & 251988.044 & 9 & 487 & 704.7 & 42 & Strong $\mathrm{CH}_{3} \mathrm{OH}$ \\
\hline 589 & $57_{9,49}-56_{9,48^{\star}}^{\star}$ & 251988.316 & 8 & 422 & 719.0 & 42 & Strong $\mathrm{CH}_{3} \mathrm{OH}$ \\
\hline 591 & $57_{13,44}-56_{13,43}{ }^{\star}$ & 252004.207 & 10 & 512 & 699.1 & 42 & Strong $\mathrm{C}_{2} \mathrm{H}_{5} \mathrm{CN}$ \\
\hline 593 & $57_{8,50}-56_{8,49}{ }^{\star}$ & 252015.263 & 9 & 405 & 722.9 & 42 & Strong $\mathrm{C}_{2} \mathrm{H}_{5} \mathrm{CN}$ and ${ }^{13} \mathrm{CH}_{2} \mathrm{CO}$ \\
\hline 595 & $57_{14,43}-56_{14,42^{\star}}$ & 252025.947 & 10 & 540 & 692.9 & 42 & Strong $\mathrm{C}_{2} \mathrm{H}_{5} \mathrm{CN}, v_{13}=1 / v_{21}=1$ \\
\hline 597 & $57_{15,42}-56_{15,41}$ ^ & 252052.483 & 11 & 570 & 686.4 & 42 & Blend with $\mathrm{CH}_{3}{ }^{13} \mathrm{CH}_{2} \mathrm{CN}$ and $U$-line \\
\hline 599 & $57_{7,51}-56_{7,50}$ & 252067.511 & 9 & 389 & 726.4 & 42 & Blend with $\mathrm{t}-\mathrm{HCOOH}$ \\
\hline 600 & $57_{7,50}-56_{7,49}$ & 252067.645 & 9 & 389 & 726.4 & 42 & Blend with $\mathrm{t}-\mathrm{HCOOH}$ \\
\hline 601 & $57_{16,41}-56_{16,40}^{\star}$ & 252083.278 & 11 & 602 & 679.4 & 42 & Strong t- $\mathrm{HCOOH}$ and $\mathrm{CH}_{3} \mathrm{OH}$ \\
\hline 603 & $57_{17,40}-56_{17,39^{\star}}$ & 252117.956 & 11 & 636 & 671.9 & 42 & Blend with $\mathrm{C}_{2} \mathrm{H}_{5} \mathrm{CN}, v_{13}=1 / v_{21}=1$ and $\mathrm{CH}_{2}(\mathrm{OH}) \mathrm{CHO}$ \\
\hline 605 & $57_{18,39}-56_{18,38^{\star}}$ & 252156.248 & 12 & 671 & 664.0 & 42 & Strong $\mathrm{C}_{2} \mathrm{H}_{5} \mathrm{CN}, v_{13}=1 / v_{21}=1$ \\
\hline 607 & $57_{6,52}-56_{6,51}$ & 252161.456 & 10 & 376 & 729.2 & 42 & Strong $\mathrm{C}_{2} \mathrm{H}_{5} \mathrm{CN}, v_{13}=1 / v_{21}=1$ \\
\hline 608 & $57_{6,51}-56_{6,50}$ & 252165.248 & 10 & 376 & 729.2 & 42 & Strong $\mathrm{C}_{2} \mathrm{H}_{5} \mathrm{CN}, v_{13}=1 / v_{21}=1$ \\
\hline 609 & $59_{1,58}-58_{2,57}$ & 252195.854 & 23 & 365 & 34.8 & 42 & Strong $\mathrm{C}_{2} \mathrm{H}_{5} \mathrm{CN}, v_{13}=1 / v_{21}=1$ \\
\hline 610 & $57_{19,38}-56_{19,37^{\star}}^{\star}$ & 252197.960 & 12 & 709 & 655.6 & 42 & Strong $\mathrm{C}_{2} \mathrm{H}_{5} \mathrm{CN}, v_{13}=1 / v_{21}=1$ \\
\hline 612 & $57_{20,37}-56_{20,36}{ }^{\star}$ & 252242.951 & 14 & 749 & 646.7 & 42 & Strong $\mathrm{C}_{2} \mathrm{H}_{5} \mathrm{CN}$ and $\mathrm{CH}_{3} \mathrm{OH}$ \\
\hline 614 & $57_{21,36}-56_{21,35^{\star}}$ & 252291.118 & 17 & 791 & 637.4 & 42 & Blend with $U$-line and $\mathrm{CH}_{3} \mathrm{CH}_{3} \mathrm{CO}, v_{t}=1$ \\
\hline 616 & $57_{5,53}-56_{5,52}$ & 252310.240 & 11 & 365 & 731.8 & 42 & $\begin{array}{l}\text { Blend with } \mathrm{CH}_{3} \mathrm{CH}_{3} \mathrm{CO}, v_{t}=1 \text { and }{ }^{13} \mathrm{CH}_{3} \mathrm{CH}_{2} \mathrm{CN} \text {, uncertain } \\
\text { baseline }\end{array}$ \\
\hline 617 & $57_{4,54}-56_{4,53}$ & 252367.776 & 11 & 355 & 733.8 & 42 & Strong $\mathrm{CH}_{3} \mathrm{OCH}_{3}$ and ${ }^{13} \mathrm{CH}_{3} \mathrm{OH}$ \\
\hline 618 & $57_{5,52}-56_{5,51}$ & 252380.765 & 11 & 365 & 731.8 & 42 & Blend with ${ }^{13} \mathrm{CH}_{3} \mathrm{CH}_{2} \mathrm{CN}$ \\
\hline 619 & $58_{2,57}-57_{2,56}$ & 253991.392 & 28 & 353 & 749.1 & 32 & Blend with $\mathrm{NS}$ and $\mathrm{CH}_{3} \mathrm{OH}$ in absorption \\
\hline 620 & $59_{1,59}-58_{1,58}$ & 255355.480 & 56 & 358 & 762.9 & 217 & Strong ${ }^{13} \mathrm{CH}_{3} \mathrm{OH}$ \\
\hline 621 & $59_{0,59}-58_{0,58}$ & 255388.370 & 56 & 358 & 762.8 & 217 & Strong OCS, $\mathrm{CH}_{2}{ }^{13} \mathrm{CHCN}$, and $\mathrm{CH}_{2} \mathrm{CH}^{13} \mathrm{CN}$ \\
\hline 622 & $58_{2,56}-57_{2,55}$ & 258190.922 & 16 & 358 & 749.2 & 1127 & Strong $\mathrm{CH}_{3} \mathrm{CN}, v_{8}=1$ and $\mathrm{C}_{2} \mathrm{H}_{3} \mathrm{CN}, v_{15}=1$ \\
\hline 623 & $59_{2,58}-58_{2,57}$ & 258304.475 & 32 & 365 & 762.1 & 1127 & Blend with $\mathrm{CH}_{3} \mathrm{CN}, v_{8}=1$ \\
\hline 624 & $59_{1,58}-58_{1,57}$ & 258877.691 & 30 & 365 & 762.2 & 1609 & Noisy \\
\hline 625 & $58_{3,55}-57_{3,54}$ & 259034.024 & 30 & 362 & 748.4 & 1609 & Strong ${ }^{13} \mathrm{CH}_{3} \mathrm{OH}$ and ${ }^{13} \mathrm{CH}_{2} \mathrm{CHCN}$ \\
\hline 626 & $59_{3,57}-58_{3,56}$ & 260422.403 & 15 & 372 & 761.3 & 413 & Strong $\mathrm{CH}_{3} \mathrm{OCHO}$ and $\mathrm{C}_{2} \mathrm{H}_{5} \mathrm{CN}$ \\
\hline 627 & $60_{1,59}-59_{1,58}$ & 263146.854 & 35 & 377 & 775.1 & 74 & Blend with ${ }^{13} \mathrm{CH}_{3} \mathrm{CH}_{2} \mathrm{CN}$, baseline problem \\
\hline 628 & $59_{3,56}-58_{3,55}$ & 263525.388 & 34 & 374 & 761.5 & 74 & Strong $\mathrm{CH}_{3} \mathrm{OCH}_{3}$ \\
\hline 629 & $16_{5,12}-15_{4,11}$ & 263780.394 & 11 & 42 & 6.3 & 108 & Strong $\mathrm{HC}_{3} \mathrm{~N}$ and $\mathrm{CH}_{3} \mathrm{OH}, v_{t}=1$ \\
\hline 630 & $16_{5,11}-15_{4,12}$ & 263780.579 & 11 & 42 & 6.3 & 108 & Strong $\mathrm{HC}_{3} \mathrm{~N}$ and $\mathrm{CH}_{3} \mathrm{OH}, v_{t}=1$ \\
\hline 631 & $61_{1,61}-60_{1,60}$ & 263943.020 & 70 & 382 & 788.7 & 108 & Blend with $\mathrm{C}_{2} \mathrm{H}_{5} \mathrm{CN}$ and $\mathrm{CH}_{3} \mathrm{CH}_{3} \mathrm{CO}$ \\
\hline 632 & $61_{0,61}-60_{0,60}$ & 263969.205 & 70 & 382 & 788.7 & 108 & Blend with $\mathrm{HC}_{3} \mathrm{~N}, v_{5}=1 / v_{7}=3, \mathrm{C}_{2} \mathrm{H}_{5} \mathrm{CN}, v_{20}=1$, and $\mathrm{CH}_{3}{ }^{13} \mathrm{CH}_{2} \mathrm{CN}$ \\
\hline 633 & $7_{6,1}-6_{5,2^{\star}}^{\star}$ & 266831.913 & 15 & 30 & 5.4 & 91 & Strong $\mathrm{CH}_{3} \mathrm{OCHO}, \mathrm{CH}_{2}{ }^{13} \mathrm{CHCN}$, and $\mathrm{CH}_{3} \mathrm{OH}$ \\
\hline 635 & $60_{2,58}-59_{2,57}$ & 266865.326 & 17 & 383 & 775.0 & 91 & Strong $\mathrm{CH}_{3} \mathrm{OH}, v_{t}=1$ \\
\hline 636 & $61_{2,60}-60_{2,59}$ & 266924.955 & 41 & 390 & 787.9 & 91 & Blend with $\mathrm{CH}_{3} \mathrm{OCHO}, \mathrm{C}_{2} \mathrm{H}_{5} \mathrm{CN}, \mathrm{C}_{2} \mathrm{H}_{3} \mathrm{CN}, \mathrm{SO}_{2}$, and $U$-line \\
\hline
\end{tabular}

Notes: ${ }^{a}$ Numbering of the observed transitions associated with a modeled line stronger than $20 \mathrm{mK} .{ }^{b}$ Transitions marked with a ${ }^{\star}$ are double with a frequency difference less than $0.1 \mathrm{MHz}$. The quantum numbers of the second one are not shown. ${ }^{c}$ Frequency uncertainty. ${ }^{d}$ Lower energy level in temperature units $\left(E_{\mathrm{l}} / k_{\mathrm{B}}\right) .{ }^{e}$ Calculated rms noise level in $T_{\mathrm{mb}}$ scale. 
A. Belloche et al.: Detection and chemical modeling of ethyl formate and $n$-propyl cyanide in Sgr B2(N), Online Material p 26

Table 10. Transitions of the gauche-conformer of $n$-propyl cyanide observed with the IRAM $30 \mathrm{~m}$ telescope toward Sgr B2(N). The horizontal lines mark discontinuities in the observed frequency coverage. Only the transitions associated with a modeled line stronger than $20 \mathrm{mK}$ are listed.

\begin{tabular}{|c|c|c|c|c|c|c|c|}
\hline$N^{a}$ & Transition $^{b}$ & $\begin{array}{c}\text { Frequency } \\
(\mathrm{MHz}) \\
(3)\end{array}$ & $\begin{array}{l}\text { Unc. }^{c} \\
(\mathrm{kHz}) \\
(4)\end{array}$ & $\begin{array}{l}E_{\mathrm{l}}^{d} \\
(\mathrm{~K}) \\
(5)\end{array}$ & $\begin{array}{l}S \mu^{2} \\
\left(\mathrm{D}^{2}\right) \\
(6)\end{array}$ & $\begin{array}{c}\sigma^{e} \\
(\mathrm{mK}) \\
(7)\end{array}$ & Comments \\
\hline 1 & $17_{9,9}-16_{9,8}{ }^{\star}$ & 101965.911 & 6 & 199 & 262.1 & 34 & Blend with $U$-line \\
\hline 3 & $17_{8,10}-16_{8,9}{ }^{\star}$ & 102048.097 & 6 & 193 & 283.5 & 34 & Blend with $\mathrm{NH}_{2} \mathrm{CHO}, v_{12}=1$ \\
\hline 5 & $29_{2,27}-29_{1,28}$ & 102048.362 & 6 & 253 & 66.0 & 34 & Blend with $\mathrm{NH}_{2} \mathrm{CHO}, v_{12}=1$ \\
\hline 6 & $34_{5,30}-34_{3,31}$ & 102175.037 & 5 & 308 & 17.0 & 30 & $\begin{array}{l}\text { Blend with } \mathrm{C}_{2} \mathrm{H}_{5} \mathrm{CN}, v_{13}=1 / v_{21}=1,{ }^{13} \mathrm{CH}_{2} \mathrm{CHCN} \text {, and } \\
\mathrm{CH}_{3} \mathrm{OCHO}, v_{\mathrm{t}}=1\end{array}$ \\
\hline 7 & $17_{7,11}-16_{7,10}$ & 102176.110 & 6 & 188 & 302.4 & 30 & $\begin{array}{l}\text { Blend with } \mathrm{C}_{2} \mathrm{H}_{5} \mathrm{CN}, v_{13}=1 / v_{21}=1,{ }^{13} \mathrm{CH}_{2} \mathrm{CHCN} \text {, and } \\
\mathrm{CH}_{3} \mathrm{OCHO}, v_{\mathrm{t}}=1\end{array}$ \\
\hline 8 & $17_{7,10}-16_{7,9}$ & 102176.872 & 6 & 188 & 302.3 & 30 & $\begin{array}{l}\text { Blend with } \mathrm{C}_{2} \mathrm{H}_{5} \mathrm{CN}, v_{13}=1 / v_{21}=1,{ }^{13} \mathrm{CH}_{2} \mathrm{CHCN} \text {, and } \\
\mathrm{CH}_{3} \mathrm{OCHO}, v_{\mathrm{t}}=1\end{array}$ \\
\hline 9 & $19_{0,19}-18_{0,18}$ & 104811.610 & 6 & 179 & 403.9 & 25 & Blend with $\mathrm{C}_{2} \mathrm{H}_{5} \mathrm{CN}, v_{13}=1 / v_{21}=1$ and $\mathrm{C}_{2} \mathrm{H}_{5} \mathrm{OH}$ \\
\hline 10 & $20_{8,12}-20_{7,13}$ & 104813.646 & 6 & 210 & 89.8 & 25 & $\begin{array}{l}\text { Blend with } \mathrm{C}_{2} \mathrm{H}_{5} \mathrm{OH}, \mathrm{C}_{2} \mathrm{H}_{3} \mathrm{CN}, v_{11}=1, \mathrm{CH}_{3}{ }^{13} \mathrm{CH}_{2} \mathrm{CN} \text {, and } \\
\mathrm{C}_{2} \mathrm{H}_{5} \mathrm{CN}, v_{13}=1 / v_{21}=1\end{array}$ \\
\hline 11 & $18_{10,8}-17_{10,7^{\star}}$ & 107933.825 & 6 & 210 & 266.5 & 46 & Blend with $\mathrm{CH}_{3} \mathrm{CH}_{3} \mathrm{CO}, v_{t}=1, \mathrm{C}_{2} \mathrm{H}_{5} \mathrm{CN}, v_{13}=1 / v_{21}=1$, and $U$-line \\
\hline 13 & $189,10-17_{9,9^{\star}}$ & 107999.345 & 6 & 204 & 289.1 & 46 & Blend with $U$-line \\
\hline 15 & $18_{8,11}-17_{8,10} \star$ & 108098.501 & 6 & 198 & 309.4 & 48 & Blend with $U$-line \\
\hline 17 & $18_{7,12}-17_{7,11}$ & 108252.103 & 6 & 193 & 327.2 & 48 & Blend with $U$-line \\
\hline 18 & $18_{7,11}-17_{7,10}$ & 108253.743 & 6 & 193 & 327.2 & 48 & Blend with $U$-line \\
\hline 19 & $18_{4,15}-17_{4,14}$ & 108520.203 & 7 & 182 & 365.9 & 20 & Blend with $\mathrm{C}_{2} \mathrm{H}_{5} \mathrm{OH}$ \\
\hline 20 & $18_{2,16}-17_{2,15}$ & 108793.066 & 7 & 179 & 375.5 & 20 & Blend with ${ }^{13} \mathrm{CN}$ in absorption and $\mathrm{C}_{2} \mathrm{H}_{5} \mathrm{CN}, v_{13}=1 / v_{21}=1$ \\
\hline 21 & $19_{2,18}-18_{2,17}$ & 108827.746 & 7 & 182 & 399.0 & 20 & Blend with ${ }^{13} \mathrm{CN}$ in absorption and $U$-line? \\
\hline 22 & $19_{1,18}-18_{1,17}$ & 109039.231 & 7 & 182 & 399.0 & 29 & Blend with $\mathrm{C}_{2} \mathrm{H}_{3} \mathrm{CN}$ and $U$-line? \\
\hline 23 & $20_{1,20}-19_{1,19}$ & 110209.409 & 6 & 184 & 425.4 & 24 & Blend with ${ }^{13} \mathrm{CO}$ and $\mathrm{HC}_{3} \mathrm{~N}, v_{5}=1 / v_{7}=3$ \\
\hline 24 & $20_{0,20}-19_{0,19}$ & 110214.195 & 6 & 184 & 425.4 & 24 & Blend with ${ }^{13} \mathrm{CO}$ and $\mathrm{HC}_{3} \mathrm{~N}, v_{5}=1 / v_{7}=3$ \\
\hline 25 & $18_{4,14}-17_{4,13}$ & 111109.974 & 6 & 182 & 366.9 & 25 & Blend with $U$-line \\
\hline 26 & $18_{3,15}-17_{3,14}$ & 112088.070 & 7 & 181 & 375.2 & 42 & Blend with $U$-line \\
\hline 27 & $19_{3,17}-18_{3,16}$ & 112370.034 & 7 & 185 & 394.3 & 42 & Blend with $\mathrm{CH}_{3} \mathrm{CH}_{3} \mathrm{CO}$ and $\mathrm{CH}_{3} \mathrm{OCH}_{3}$ \\
\hline 28 & $19_{15,4}-18_{15,3}{ }^{\star}$ & 113832.488 & 7 & 258 & 153.3 & 34 & Blend with $\mathrm{C}_{2} \mathrm{H}_{3} \mathrm{CN}$ and $U$-line \\
\hline 30 & $19_{16,3}-18_{16,2}{ }^{\star}$ & 113833.361 & 7 & 268 & 118.4 & 34 & Blend with $\mathrm{C}_{2} \mathrm{H}_{3} \mathrm{CN}$ and $U$-line \\
\hline 32 & $19_{12,7}-18_{12,6}{ }^{\star}$ & 113872.326 & 6 & 230 & 244.6 & 34 & Blend with $\mathrm{C}_{2} \mathrm{H}_{5} \mathrm{OH}$ and $U$-line \\
\hline 34 & $19_{11,8}-18_{11,7^{\star}}$ & 113907.356 & 6 & 223 & 270.5 & 34 & Strong $\mathrm{C}_{2} \mathrm{H}_{3} \mathrm{CN}$ \\
\hline 36 & $19_{10,10}-18_{10,9^{\star}}$ & 113960.196 & 6 & 216 & 294.2 & 33 & Blend with $\mathrm{C}_{2} \mathrm{H}_{3} \mathrm{CN}$ \\
\hline 38 & $19_{9,11}-18_{9,10} \star$ & 114038.886 & 6 & 209 & 315.7 & 33 & Blend with $U$-line \\
\hline 40 & $19_{2,17}-18_{2,16}$ & 114077.162 & 7 & 184 & 396.3 & 33 & Blend with $\mathrm{C}_{2} \mathrm{H}_{3} \mathrm{CN}, v_{15}=1$ and $U$-line \\
\hline 41 & $19_{8,12}-18_{8,11}$ & 114157.197 & 6 & 203 & 334.8 & 33 & Blend with $U$-line \\
\hline 42 & $19_{8,11}-18_{8,10}$ & 114157.321 & 6 & 203 & 334.8 & 33 & Blend with $U$-line \\
\hline 43 & $20_{2,19}-19_{2,18}$ & 114255.098 & 6 & 187 & 420.4 & 33 & Blend with $\mathrm{C}_{2} \mathrm{H}_{3} \mathrm{CN}, v_{11}=1$ \\
\hline 44 & $19_{7,13}-18_{7,12}$ & 114339.524 & 6 & 198 & 351.7 & 33 & Blend with $\mathrm{C}_{2} \mathrm{H}_{3} \mathrm{CN}, v_{11}=1$ and $\mathrm{NH}_{2} \mathrm{CHO}$ \\
\hline 45 & $20_{1,19}-19_{1,18}$ & 114399.053 & 6 & 187 & 420.3 & 33 & Blend with $\mathrm{C}_{2} \mathrm{H}_{5} \mathrm{CN}, v_{13}=1 / v_{21}=1$ and $U$-line? \\
\hline 46 & $19_{4,16}-18_{4,15}$ & 114451.328 & 7 & 187 & 388.1 & 37 & Blend with $\mathrm{C}_{2} \mathrm{H}_{3} \mathrm{CN}, v_{11}=1 / v_{15}=1$ and $c-\mathrm{C}_{2} \mathrm{H}_{4} \mathrm{O}$ \\
\hline 47 & $19_{6,14}-18_{6,13}$ & 114615.647 & 6 & 194 & 366.3 & 37 & Strong $\mathrm{H}^{13} \mathrm{CCCN}$ and $\mathrm{C}_{2} \mathrm{H}_{3} \mathrm{CN}$ \\
\hline 48 & $19_{6,13}-18_{6,12}$ & 114676.990 & 6 & 194 & 366.4 & 37 & Blend with $\mathrm{H}^{13} \mathrm{CCCN}, v_{5}=1 / v_{7}=3$ and $U$-line \\
\hline 49 & $20_{2,19}-19_{1,18}$ & 114679.259 & 7 & 187 & 130.0 & 37 & Blend with $\mathrm{H}^{13} \mathrm{CCCN}, v_{5}=1 / v_{7}=3$ and $U$-line \\
\hline 50 & $19_{5,15}-18_{5,14}$ & 114881.716 & 6 & 190 & 378.6 & 59 & Blend with $\mathrm{C}_{2} \mathrm{H}_{3} \mathrm{CN}, v_{11}=3$ and $\mathrm{CH}_{2}(\mathrm{OH}) \mathrm{CHO}$ \\
\hline 51 & $19_{5,14}-18_{5,13}$ & 115556.072 & 6 & 190 & 378.7 & 60 & Blend with $\mathrm{C}_{2} \mathrm{H}_{3} \mathrm{CN}$, NS, and $\mathrm{CH}_{3} \mathrm{OCHO}, v_{\mathrm{t}}=1$ \\
\hline 52 & $21_{0,21}-20_{1,20}$ & 115609.125 & 6 & 189 & 174.8 & 79 & Blend with $\mathrm{CH}_{2} \mathrm{NH}$ and $U$-line? \\
\hline 53 & $21_{1,21}-20_{1,20}$ & 115613.838 & 6 & 189 & 446.8 & 79 & Blend with $\mathrm{CH}_{2} \mathrm{NH}$ and $\mathrm{CH}_{3} \mathrm{CHO}$ \\
\hline 54 & $21_{0,21}-20_{0,20}$ & 115616.804 & 6 & 189 & 446.8 & 79 & Blend with $\mathrm{CH}_{2} \mathrm{NH}$ and $\mathrm{CH}_{3} \mathrm{CHO}$ \\
\hline 55 & $24_{5,20}-23_{5,19}$ & 145147.268 & 6 & 221 & 491.0 & 25 & Strong $\mathrm{C}_{2} \mathrm{H}_{3} \mathrm{CN}$ \\
\hline 56 & $25_{3,23}-24_{3,22}$ & 145354.256 & 6 & 221 & 522.8 & 25 & Blend with $\mathrm{HC}_{3} \mathrm{~N}, v_{4}=1$ \\
\hline 57 & $24_{6,19}-23_{6,18}$ & 145359.467 & 6 & 224 & 481.8 & 25 & Blend with $\mathrm{HC}_{3} \mathrm{~N}, v_{4}=1$ \\
\hline 58 & $26_{4,23}-25_{4,22}$ & 154516.394 & 5 & 232 & 540.1 & 112 & Blend with $\mathrm{NH}_{2} \mathrm{CH}_{2} \mathrm{CN}$ and $\mathrm{C}_{2} \mathrm{H}_{5} \mathrm{CN}, v_{13}=1 / v_{21}=1$ \\
\hline 59 & $27_{7,21}-26_{7,20}$ & 163471.366 & 5 & 250 & 539.4 & 38 & Blend with $\mathrm{HCC}^{13} \mathrm{CN}, v_{7}=1$ and $\mathrm{NH}_{2} \mathrm{CH}_{2} \mathrm{CN}$ \\
\hline 60 & $12_{7,6}-11_{6,5}$ & 163626.490 & 12 & 164 & 62.4 & 38 & Blend with a- $\mathrm{C}_{3} \mathrm{H}_{7} \mathrm{CN}$ and $U$-line \\
\hline 61 & $12_{7,5}-11_{6,6}$ & 163626.670 & 12 & 164 & 62.4 & 38 & Blend with a- $\mathrm{C}_{3} \mathrm{H}_{7} \mathrm{CN}$ and $U$-line \\
\hline 62 & $27_{6,22}-26_{6,21}$ & 163729.422 & 5 & 246 & 549.4 & 38 & Blend with a- $\mathrm{C}_{3} \mathrm{H}_{7} \mathrm{CN}$ and $U$-line \\
\hline 63 & $28_{3,25}-27_{3,24}$ & 166501.674 & 5 & 247 & 583.3 & 66 & Blend with $\mathrm{C}_{2} \mathrm{H}_{5} \mathrm{CN}, v_{13}=1 / v_{21}=1$ and $\mathrm{CH}_{3} \mathrm{CN}, v_{8}=2$ \\
\hline 64 & $30_{3,28}-29_{2,27}$ & 172509.803 & 5 & 258 & 186.7 & 44 & Strong $\mathrm{C}_{2} \mathrm{H}_{5} \mathrm{CN}, v_{13}=1 / v_{21}=1$ \\
\hline 65 & $24_{13,11}-24_{12,12^{\star}}$ & 176027.815 & 8 & 268 & 88.7 & 365 & Blend with $\mathrm{HNCO}, v_{5}=1$ and ${ }^{13} \mathrm{CH}_{2} \mathrm{CO}$ \\
\hline 67 & $23_{13,10}-23_{12,11}$ * & 176085.591 & 8 & 261 & 82.2 & 365 & Blend with $\mathrm{CH}_{3} \mathrm{OCH}_{3}$ \\
\hline 69 & $22_{13,9}-22_{12,10}^{\star}$ & 176134.711 & 8 & 254 & 75.7 & 365 & Blend with $\mathrm{HNCO}, v_{6}=1$ and $\mathrm{HNCO}, v_{5}=1$ \\
\hline 71 & $37_{0,37}-36_{1,36}{ }^{\star}$ & 201966.900 & 11 & 309 & 321.5 & 138 & Blend with $\mathrm{CH}_{3} \mathrm{CN}$ \\
\hline 73 & $37_{0,37}-36_{0,36}{ }^{\star}$ & 201966.902 & 11 & 309 & 789.3 & 138 & Blend with $\mathrm{CH}_{3} \mathrm{CN}$ \\
\hline 75 & $33_{5,29}-32_{4,28}$ & 201985.474 & 6 & 293 & 131.9 & 138 & Blend with $\mathrm{CH}_{3} \mathrm{OH}$ \\
\hline
\end{tabular}


Table 10. continued.

\begin{tabular}{|c|c|c|c|c|c|c|c|}
\hline$N^{a}$ & Transition $^{b}$ & $\begin{array}{c}\text { Frequency } \\
\text { (MHz) } \\
\text { (3) }\end{array}$ & $\begin{array}{l}\text { Unc. }^{c} \\
(\mathrm{kHz}) \\
(4)\end{array}$ & $\begin{array}{l}E_{1}^{d} \\
(\mathrm{~K}) \\
(5)\end{array}$ & $\begin{array}{l}S \mu^{2} \\
\left(\mathrm{D}^{2}\right) \\
(6)\end{array}$ & $\begin{array}{c}\sigma^{e} \\
(\mathrm{mK}) \\
(7)\end{array}$ & Comments \\
\hline 76 & $34_{5,30}-33_{5,29}$ & 202090.888 & 6 & 303 & 707.1 & 138 & Strong $\mathrm{CH}_{2} \mathrm{CO}$ \\
\hline 77 & $56_{3,53}-56_{3,54}{ }^{\star}$ & 202474.090 & 224 & 572 & 15.7 & 108 & Blend with $U$-line and $\mathrm{H}_{2} \mathrm{CS}$ \\
\hline 79 & $56_{4,53}-56_{3,54}^{\star}$ & 202474.109 & 224 & 572 & 100.1 & 108 & Blend with $U$-line and $\mathrm{H}_{2} \mathrm{CS}$ \\
\hline 81 & $41_{15,26}-41_{14,27}{ }^{\star}$ & 202477.934 & 26 & 447 & 185.2 & 108 & Blend with $U$-line and $\mathrm{H}_{2} \mathrm{CS}$ \\
\hline 83 & $40_{15,25}-40_{14,26}$ * & 202689.659 & 25 & 435 & 178.9 & 108 & Blend with $\mathrm{C}_{2} \mathrm{H}_{3} \mathrm{CN}, v_{15}=1$ and $\mathrm{NH}_{2} \mathrm{CHO}$ \\
\hline 85 & $33_{7,26}-32_{7,25}$ & 202881.421 & 5 & 302 & 675.4 & 138 & Blend with $\mathrm{C}_{2} \mathrm{H}_{3} \mathrm{CN}, v_{11}=1$ \\
\hline 86 & $39_{15,24}-39_{14,25}$ * & 202883.694 & 24 & 423 & 172.6 & 138 & Blend with $\mathrm{C}_{2} \mathrm{H}_{3} \mathrm{CN}, v_{11}=1$ \\
\hline 88 & $38_{15,23}-38_{14,24}$ * & 203061.073 & 23 & 412 & 166.3 & 138 & Blend with ${ }^{13} \mathrm{CH}_{3} \mathrm{CH}_{2} \mathrm{CN}$ and $\mathrm{C}_{2} \mathrm{H}_{5} \mathrm{OCHO}$ \\
\hline 90 & $34_{4,30}-33_{4,29}$ & 203180.785 & 6 & 303 & 707.8 & 138 & Blend with $\mathrm{C}_{2} \mathrm{H}_{3} \mathrm{CN}, v_{11}=2$ \\
\hline 91 & $37_{15,22}-37_{14,23}{ }^{\star}$ & 203222.780 & 23 & 401 & 160.0 & 138 & Blend with ${ }^{34} \mathrm{SO}_{2}$ \\
\hline 93 & $35_{3,32}-34_{4,31}$ & 203290.077 & 6 & 308 & 195.3 & 161 & Blend with $\mathrm{C}_{2} \mathrm{H}_{5} \mathrm{OCHO}, \mathrm{CH}_{3} \mathrm{CN}, v_{8}=2$, and $U$-line \\
\hline 94 & $36_{15,21}-36_{14,22}{ }^{\star}$ & 203369.755 & 22 & 390 & 153.7 & 161 & Strong $\mathrm{CH}_{3} \mathrm{OCH}_{3}$ \\
\hline 96 & $35_{4,32}-34_{4,31}$ & 203466.519 & 6 & 308 & 732.0 & 161 & Blend with $\mathrm{CH}_{3} \mathrm{CN}, v_{8}=2$ and $\mathrm{CH}_{3} \mathrm{OCHO}$ \\
\hline 97 & $35_{15,20}-35_{14,21}$ * & 203502.897 & 22 & 380 & 147.5 & 161 & Blend with ${ }^{34} \mathrm{SO}_{2}$ \\
\hline 99 & $35_{3,32}-34_{3,31}$ & 203552.465 & 6 & 308 & 732.0 & 161 & Blend with $\mathrm{CH}_{3} \mathrm{CN}, v_{8}=2$ and $\mathrm{H}^{13} \mathrm{CCCN}, v_{7}=1$ \\
\hline 100 & $34_{15,19}-34_{14,20}$ * & 203623.068 & 22 & 370 & 141.2 & 161 & Blend with $\mathrm{CH}_{3} \mathrm{OCH}_{3}$ \\
\hline 102 & $35_{4,32}-34_{3,31}$ & 203728.907 & 6 & 308 & 195.4 & 364 & Blend with $\mathrm{C}_{2} \mathrm{H}_{5} \mathrm{CN}, v_{13}=1 / v_{21}=1$ and $U$-line \\
\hline 103 & $33_{15,18}-33_{14,19}$ * & 203731.092 & 21 & 360 & 135.0 & 364 & Blend with $\mathrm{C}_{2} \mathrm{H}_{5} \mathrm{CN}, v_{13}=1 / v_{21}=1$ and $U$-line \\
\hline 105 & $34_{20,14}-33_{20,13}$ * & 203795.929 & 10 & 429 & 476.3 & 364 & Strong $\mathrm{C}_{2} \mathrm{H}_{3} \mathrm{CN}$ \\
\hline 107 & $34_{21,13}-33_{21,12}$ * & 203796.393 & 10 & 443 & 450.5 & 364 & Strong $\mathrm{C}_{2} \mathrm{H}_{3} \mathrm{CN}$ \\
\hline 109 & $34_{22,12}-33_{22,11}$ * & 203805.898 & 11 & 457 & 423.4 & 364 & Strong $\mathrm{C}_{2} \mathrm{H}_{3} \mathrm{CN}$ \\
\hline 111 & $34_{19,15}-33_{19,14^{\star}}$ & 203805.992 & 10 & 416 & 500.8 & 364 & Strong $\mathrm{C}_{2} \mathrm{H}_{3} \mathrm{CN}$ \\
\hline 113 & $34_{23,11}-33_{23,10}$ * & 203823.272 & 12 & 473 & 395.0 & 364 & Blend with $\mathrm{C}_{2} \mathrm{H}_{3} \mathrm{CN},{ }^{13} \mathrm{CH}_{3} \mathrm{CH}_{2} \mathrm{CN}$, and $\mathrm{C}_{2} \mathrm{H}_{5} \mathrm{CN}$ \\
\hline 115 & $32_{15,17}-32_{14,18}$ * & 203827.761 & 21 & 351 & 128.7 & 364 & Blend with $\mathrm{C}_{2} \mathrm{H}_{3} \mathrm{CN},{ }^{13} \mathrm{CH}_{3} \mathrm{CH}_{2} \mathrm{CN}$, and $\mathrm{C}_{2} \mathrm{H}_{5} \mathrm{CN}$ \\
\hline 117 & $34_{18,16}-33_{18,15^{\star}}$ & 203828.503 & 10 & 403 & 524.2 & 364 & Blend with $\mathrm{C}_{2} \mathrm{H}_{3} \mathrm{CN},{ }^{13} \mathrm{CH}_{3} \mathrm{CH}_{2} \mathrm{CN}$, and $\mathrm{C}_{2} \mathrm{H}_{5} \mathrm{CN}$ \\
\hline 119 & $14_{9,6}-13_{8,5}{ }^{\star}$ & 203835.897 & 12 & 180 & 80.1 & 364 & Blend with $\mathrm{C}_{2} \mathrm{H}_{3} \mathrm{CN},{ }^{13} \mathrm{CH}_{3} \mathrm{CH}_{2} \mathrm{CN}$, and $\mathrm{C}_{2} \mathrm{H}_{5} \mathrm{CN}$ \\
\hline 121 & $34_{24,10}-33_{24,9}$ * & 203847.587 & 14 & 488 & 365.4 & 364 & Blend with $\mathrm{CH}_{3} \mathrm{OCHO}$ and $\mathrm{C}_{2} \mathrm{H}_{5} \mathrm{OH}$ \\
\hline 123 & $65_{14,52}-64_{15,49}$ & 203856.381 & 379 & 809 & 80.4 & 364 & Blend with $\mathrm{C}_{2} \mathrm{H}_{5} \mathrm{OH}$ and $\mathrm{CH}_{3} \mathrm{OCHO}$ \\
\hline 124 & $34_{17,17}-33_{17,16^{\star}}$ & 203865.981 & 10 & 392 & 546.2 & 364 & Blend with $\mathrm{CH}_{3} \mathrm{OCHO}$ \\
\hline 126 & $34_{25,9^{-}}-33_{25,8^{\star}}$ & 203878.090 & 15 & 505 & 334.5 & 364 & Blend with $\mathrm{CH}_{3} \mathrm{CH}_{3} \mathrm{CO}$ and $U$-line \\
\hline 128 & $31_{15,16}-31_{14,17^{\star}}$ & 203913.834 & 21 & 342 & 122.4 & 364 & Strong $\mathrm{C}_{2} \mathrm{H}_{5} \mathrm{CN}, v_{13}=1 / v_{21}=1$ \\
\hline 130 & $34_{26,8}-33_{26,7^{\star}}$ & 203914.173 & 17 & 522 & 302.4 & 364 & Strong $\mathrm{C}_{2} \mathrm{H}_{5} \mathrm{CN}, v_{13}=1 / v_{21}=1$ \\
\hline 132 & $34_{16,18}-33_{16,17^{\star}}$ & 203921.785 & 10 & 380 & 567.0 & 364 & Strong $\mathrm{C}_{2} \mathrm{H}_{5} \mathrm{CN}, v_{13}=1 / v_{21}=1$ \\
\hline 134 & $34_{27,7}-33_{27,6^{\star}}$ & 203955.332 & 20 & 540 & 269.0 & 364 & Blend with $U$-line \\
\hline 136 & $33_{5,28}-32_{5,27}$ & 203957.407 & 6 & 296 & 687.9 & 364 & Blend with $U$-line \\
\hline 137 & $30_{15,15}-30_{14,16}$ * & 203990.040 & 20 & 333 & 116.1 & 364 & Blend with $\mathrm{C}_{2} \mathrm{H}_{5} \mathrm{CN}$ and $\mathrm{C}_{2} \mathrm{H}_{3} \mathrm{CN}, v_{11}=3$ \\
\hline 139 & $34_{15,19}-33_{15,18}$ * & 204000.498 & 9 & 370 & 586.5 & 364 & Strong $\mathrm{C}_{2} \mathrm{H}_{3} \mathrm{CN}, v_{11}=3$ and $\mathrm{C}_{2} \mathrm{H}_{3} \mathrm{CN}, v_{11}=2$ \\
\hline 141 & $34_{28,6}-33_{28,5} \star$ & 204001.151 & 22 & 558 & 234.3 & 364 & Strong $\mathrm{C}_{2} \mathrm{H}_{3} \mathrm{CN}, v_{11}=3$ and $\mathrm{C}_{2} \mathrm{H}_{3} \mathrm{CN}, v_{11}=2$ \\
\hline 143 & $34_{29,5}-33_{29,4}{ }^{\star}$ & 204051.281 & 26 & 577 & 198.4 & 364 & Blend with $\mathrm{H}^{13} \mathrm{CCCN}, v_{7}=2$ and $U$-line \\
\hline 145 & $29_{15,14}-29_{14,15}$ * & 204057.079 & 20 & 324 & 109.7 & 364 & Blend with $\mathrm{H}^{13} \mathrm{CCCN}, v_{7}=2$ and $U$-line \\
\hline 147 & $64_{6,58}-64_{6,59}$ & 204063.746 & 316 & 734 & 27.7 & 364 & Blend with $\mathrm{H}^{13} \mathrm{CCCN}, v_{7}=2$ and $U$-line \\
\hline 148 & $64_{6,58}-64_{5,59}$ & 204064.065 & 316 & 734 & 201.8 & 364 & Blend with $\mathrm{H}^{13} \mathrm{CCCN}, v_{7}=2$ and $U$-line \\
\hline 149 & $34_{30,4}-33_{30,3}{ }^{\star}$ & 204105.426 & 30 & 597 & 161.3 & 316 & Blend with $U$-line \\
\hline 151 & $34_{14,20}-33_{14,19}{ }^{\star}$ & 204108.522 & 9 & 360 & 604.7 & 316 & Blend with $U$-line \\
\hline 153 & $28_{15,13}-28_{14,14}$ * & 204115.622 & 20 & 316 & 103.3 & 316 & Blend with $U$-line \\
\hline 155 & $34_{31,3}-33_{31,2}{ }^{\star}$ & 204163.336 & 36 & 618 & 122.8 & 316 & Strong $\mathrm{CH}_{3} \mathrm{OCH}_{3}, \mathrm{C}_{2} \mathrm{H}_{3} \mathrm{CN}, v_{11}=1$, and ${ }^{13} \mathrm{CH}_{2} \mathrm{CHCN}$ \\
\hline 157 & $27_{15,12}-27_{14,13}{ }^{\star}$ & 204166.316 & 20 & 308 & 96.9 & 316 & Strong $\mathrm{CH}_{3} \mathrm{OCH}_{3}, \mathrm{C}_{2} \mathrm{H}_{3} \mathrm{CN}, v_{11}=1$, and ${ }^{13} \mathrm{CH}_{2} \mathrm{CHCN}$ \\
\hline 159 & $22_{6,17}-21_{5,16}$ & 204205.122 & 12 & 208 & 60.2 & 316 & Blend with $\mathrm{CH}_{3} \mathrm{CH}_{3} \mathrm{CO}$ and $U$-line \\
\hline 160 & $26_{15,11}-26_{14,12}$ * & 204209.781 & 20 & 300 & 90.4 & 316 & Blend with $\mathrm{CH}_{3} \mathrm{CH}_{3} \mathrm{CO}$ and $U$-line \\
\hline 162 & $25_{15,10}-25_{14,11}{ }^{\star}$ & 204246.611 & 20 & 292 & 83.8 & 316 & Strong $\mathrm{SO}_{2}$ \\
\hline 164 & 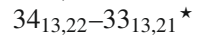 & 204255.076 & 8 & 351 & 621.9 & 316 & Strong $\mathrm{SO}_{2}$ \\
\hline 166 & $24_{15,9}-24_{14,10} \star$ & 204277.378 & 20 & 285 & 77.2 & 316 & Blend with $\mathrm{CH}_{3} \mathrm{CH}_{3} \mathrm{CO}$ and \\
\hline 168 & $52_{1,51}-52_{0,52}^{\star}$ & 204278.834 & 248 & 495 & 33.4 & 316 & Blend with $\mathrm{CH}_{3} \mathrm{CH}_{3} \mathrm{CO}$ and $U$-line \\
\hline 170 & $52_{2,51}-52_{1,52}{ }^{\star}$ & 204278.834 & 248 & 495 & 33.4 & 316 & Blend with $\mathrm{CH}_{3} \mathrm{CH}_{3} \mathrm{CO}$ and $U$-line \\
\hline 172 & $23_{15,8}-23_{14,9}{ }^{\star}$ & 204302.630 & 20 & 278 & 70.4 & 316 & Strong $\mathrm{C}_{2} \mathrm{H}_{5} \mathrm{CN}$ \\
\hline 174 & $22_{15,7}-22_{14,8}{ }^{\star}$ & 204322.890 & 20 & 272 & 63.5 & 316 & Blend with $\mathrm{C}_{2} \mathrm{H}_{5} \mathrm{CN}$ and $U$-line \\
\hline 176 & $21_{15,6}-21_{14,7}^{\star}$ & 204338.662 & 20 & 265 & 56.5 & 316 & Blend with $\mathrm{C}_{2} \mathrm{H}_{5} \mathrm{CN}, v_{13}=1 / v_{21}=1$ and $U$-line \\
\hline 178 & $20_{15,5}-20_{14,6}{ }^{\star}$ & 204350.423 & 21 & 259 & 49.3 & 316 & Blend with $\mathrm{CH}_{3} \mathrm{CH}_{3} \mathrm{CO}, v_{t}=1$ \\
\hline 180 & $19_{15,4}-19_{14,5}^{\star}$ & 204358.632 & 21 & 253 & 41.9 & 316 & Blend with $\mathrm{CH}_{3} \mathrm{CH}_{3} \mathrm{CO}$ \\
\hline 182 & $18_{15,3}-18_{14,4}^{\star}$ & 204363.725 & 21 & 248 & 34.2 & 316 & Blend with $\mathrm{CH}_{3} \mathrm{CH}_{3} \mathrm{CO}$ and $\mathrm{C}_{2} \mathrm{H}_{5} \mathrm{OCHO}$ \\
\hline 184 & $15_{15,0}-15_{14,1}^{\star}$ & 204364.340 & 22 & 233 & 9.2 & 316 & Blend with $\mathrm{CH}_{3} \mathrm{CH}_{3} \mathrm{CO}$ and $\mathrm{C}_{2} \mathrm{H}_{5} \mathrm{OCHO}$ \\
\hline 186 & $17_{15,2}-17_{14,3^{\star}}$ & 204366.116 & 21 & 243 & 26.3 & 316 & Blend with $\mathrm{CH}_{3} \mathrm{CH}_{3} \mathrm{CO}$ and $\mathrm{C}_{2} \mathrm{H}_{5} \mathrm{OCHO}$ \\
\hline 188 & $16_{15,1}-16_{14,2}{ }^{\star}$ & 204366.197 & 22 & 238 & 18.0 & 316 & Blend with $\mathrm{CH}_{3} \mathrm{CH}_{3} \mathrm{CO}$ and $\mathrm{C}_{2} \mathrm{H}_{5} \mathrm{OCHO}$ \\
\hline 190 & $60_{12,49}-59_{13,46}$ & 204450.542 & 260 & 701 & 72.3 & 316 & Blend with $\mathrm{CH}_{3} \mathrm{CH}_{3} \mathrm{CO}$ and $U$-line \\
\hline
\end{tabular}


Table 10. continued.

\begin{tabular}{|c|c|c|c|c|c|c|c|}
\hline$N^{a}$ & Transition $^{b}$ & $\begin{array}{c}\text { Frequency } \\
\text { (MHz) } \\
\text { (3) }\end{array}$ & $\begin{array}{c}\text { Unc. }^{c} \\
(\mathrm{kHz}) \\
(4)\end{array}$ & $\begin{array}{l}E_{1}^{d} \\
(\mathrm{~K}) \\
(5)\end{array}$ & $\begin{array}{l}S \mu^{2} \\
\left(\mathrm{D}^{2}\right) \\
(6)\end{array}$ & $\begin{array}{c}\sigma^{e} \\
(\mathrm{mK}) \\
(7)\end{array}$ & Comments \\
\hline 191 & $34_{12,23}-33_{12,22}{ }^{\star}$ & 204453.939 & 8 & 343 & 637.5 & 316 & Blend with $\mathrm{CH}_{3} \mathrm{CH}_{3} \mathrm{CO}$ and $U$-line \\
\hline 193 & $34_{10,25}-33_{10,24}$ & 205107.534 & 6 & 328 & 665.3 & 100 & Strong $\mathrm{CH}_{3} \mathrm{OCH}_{3}, \mathrm{C}_{2} \mathrm{H}_{5}{ }^{13} \mathrm{CN}$, and $\mathrm{CH}_{3}{ }^{13} \mathrm{CH}_{2} \mathrm{CN}$ \\
\hline 194 & $34_{10,24}-33_{10,23}$ & 205113.060 & 6 & 328 & 665.2 & 100 & Strong $\mathrm{CH}_{3} \mathrm{OCH}_{3}, \mathrm{C}_{2} \mathrm{H}_{5}{ }^{13} \mathrm{CN}$, and $\mathrm{CH}_{3}{ }^{13} \mathrm{CH}_{2} \mathrm{CN}$ \\
\hline 195 & $34_{6,29}-33_{6,28}$ & 205176.196 & 6 & 307 & 703.2 & 100 & Blend with $\mathrm{CH}_{3} \mathrm{CHO}$ and $\mathrm{CH}_{3}{ }^{13} \mathrm{CH}_{2} \mathrm{CN}$ \\
\hline 196 & $35_{4,31}-34_{5,30}$ & 205501.651 & 7 & 313 & 152.2 & 100 & Strong $\mathrm{CH}_{3} \mathrm{OCHO}$ and $\mathrm{c}-\mathrm{C}_{2} \mathrm{H}_{4} \mathrm{O}$ \\
\hline 197 & $34_{9,26}-33_{9,25}$ & 205639.058 & 6 & 322 & 677.2 & 271 & Strong $\mathrm{CH}_{3}{ }^{13} \mathrm{CH}_{2} \mathrm{CN}$ and $\mathrm{C}_{2} \mathrm{H}_{5} \mathrm{CN}$ \\
\hline 198 & $34_{9,25}-33_{9,24}$ & 205708.710 & 6 & 322 & 677.2 & 271 & Strong $\mathrm{C}_{2} \mathrm{H}_{5}{ }^{13} \mathrm{CN}$ and $\mathrm{CH}_{3} \mathrm{OCH}_{3}$ \\
\hline 199 & $33_{6,27}-32_{6,26}$ & 205773.682 & 6 & 299 & 685.9 & 271 & Blend with $U$-line and $\mathrm{CH}_{3} \mathrm{OH}$ in absorption \\
\hline 200 & $12_{10,2}-11_{9,3}{ }^{\star}$ & 205968.115 & 12 & 179 & 87.1 & 271 & Blend with $\mathrm{CH}_{3} \mathrm{CH}_{3} \mathrm{CO}, v_{t}=1$ and $\mathrm{CH}_{3} \mathrm{COOH}$ \\
\hline 202 & $37_{1,36}-36_{2,35}$ & 206014.847 & 7 & 315 & 287.3 & 280 & Strong $\mathrm{CH}_{3} \mathrm{OH}$ and $\mathrm{C}_{2} \mathrm{H}_{5} \mathrm{CN}, v_{13}=1 / v_{21}=1$ \\
\hline 203 & $37_{2,36}-36_{2,35}{ }^{\star}$ & 206014.958 & 7 & 315 & 783.9 & 280 & Strong $\mathrm{CH}_{3} \mathrm{OH}$ and $\mathrm{C}_{2} \mathrm{H}_{5} \mathrm{CN}, v_{13}=1 / v_{21}=1$ \\
\hline 205 & $37_{2,36}-36_{1,35}$ & 206015.138 & 7 & 315 & 287.3 & 280 & Strong $\mathrm{CH}_{3} \mathrm{OH}$ and $\mathrm{C}_{2} \mathrm{H}_{5} \mathrm{CN}, v_{13}=1 / v_{21}=1$ \\
\hline 206 & $345,30-33_{4,29}$ & 206035.987 & 6 & 303 & 142.4 & 280 & Strong $\mathrm{C}_{2} \mathrm{H}_{5} \mathrm{CN}, v_{13}=1 / v_{21}=1$ and $\mathrm{C}_{2} \mathrm{H}_{5} \mathrm{CN}$ \\
\hline 207 & $34_{8,27}-33_{8,26}$ & 206266.954 & 6 & 316 & 687.9 & 280 & Strong $\mathrm{C}_{2} \mathrm{H}_{5} \mathrm{CN}, v_{20}=1, \mathrm{C}_{2} \mathrm{H}_{5} \mathrm{CN}$, and $\mathrm{CH}_{3} \mathrm{OCHO}$ \\
\hline 208 & $34_{7,28}-33_{7,27}$ & 206461.559 & 6 & 312 & 696.8 & 106 & Strong $\mathrm{C}_{2} \mathrm{H}_{5} \mathrm{CN}, v_{13}=1 / v_{21}=1$ \\
\hline 209 & $34_{8,26}-33_{8,25}$ & 206887.489 & 6 & 316 & 687.9 & 106 & Blend with $\mathrm{C}_{2} \mathrm{H}_{5} \mathrm{CN}$ \\
\hline 210 & $38_{0,38}-37_{1,37^{\star}}$ & 207355.545 & 13 & 318 & 330.6 & 117 & Blend with ${ }^{13} \mathrm{CH}_{2} \mathrm{CHCN}$ and $\mathrm{CH}_{3} \mathrm{COOH}$ \\
\hline 212 & $38_{0,38}-37_{0,37^{\star}}$ & 207355.546 & 13 & 318 & 810.7 & 117 & Blend with ${ }^{13} \mathrm{CH}_{2} \mathrm{CHCN}$ and $\mathrm{CH}_{3} \mathrm{COOH}$ \\
\hline 214 & $35_{5,31}-34_{5,30}$ & 207543.638 & 6 & 313 & 728.4 & 282 & Blend with $\mathrm{CH}_{3} \mathrm{OCHO}$ and $U$-line \\
\hline 215 & $17_{8,10}-16_{7,9}$ & 207551.813 & 12 & 188 & 75.2 & 282 & Blend with $U$-line \\
\hline 216 & $17_{8,9}-16_{7,10}$ & 207552.401 & 12 & 188 & 75.2 & 282 & Blend with $U$-line \\
\hline 217 & $35_{4,31}-34_{4,30}$ & 208356.853 & 6 & 313 & 728.8 & 168 & Strong $\mathrm{HC}^{13} \mathrm{CCN}$ and $\mathrm{HCC}^{13} \mathrm{CN}$ \\
\hline 218 & $36_{3,33}-35_{4,32}$ & 208729.722 & 6 & 318 & 204.9 & 160 & Blend with $\mathrm{CH}_{3} \mathrm{OCHO}$ and $U$-line \\
\hline 219 & $36_{4,33}-35_{4,32}$ & 208847.713 & 6 & 318 & 753.3 & 160 & Strong $\mathrm{HC}^{13} \mathrm{CCN}, v_{6}=1$ \\
\hline 220 & $36_{3,33}-35_{3,32}$ & 208906.164 & 6 & 318 & 753.4 & 160 & Strong $\mathrm{C}_{2} \mathrm{H}_{3} \mathrm{CN}$ and $\mathrm{CH}_{3} \mathrm{OCHO}$ \\
\hline 221 & $36_{4,33}-35_{3,32}$ & 209024.156 & 6 & 318 & 204.9 & 160 & Strong $\mathrm{C}_{2} \mathrm{H}_{3} \mathrm{CN}$ and $\mathrm{CH}_{3} \mathrm{OCHO}$ \\
\hline 222 & $34_{5,29}-33_{5,28}$ & 209279.146 & 6 & 306 & 708.3 & 58 & Blend with $\mathrm{NH}_{2} \mathrm{CH}_{2} \mathrm{CN}$ and $\mathrm{CH}_{3} \mathrm{CH}_{3} \mathrm{CO}$ \\
\hline 223 & $34_{7,27}-33_{7,26}$ & 209670.004 & 6 & 312 & 698.1 & 45 & Blend with $\mathrm{HCC}^{13} \mathrm{CN}, v_{7}=2$ and $\mathrm{C}_{2} \mathrm{H}_{3} \mathrm{CN}, v_{11}=1$ \\
\hline 224 & $35_{21,14}-34_{21,13}{ }^{\star}$ & 209787.936 & 10 & 453 & 479.8 & 45 & Blend with $\mathrm{C}_{2} \mathrm{H}_{3} \mathrm{CN}, v_{11}=1 / v_{15}=1$ \\
\hline 226 & $35_{20,15}-34_{20,14}$ * & 209790.453 & 10 & 439 & 504.9 & 45 & Blend with $\mathrm{C}_{2} \mathrm{H}_{3} \mathrm{CN}, v_{11}=1 / v_{15}=1$ \\
\hline 228 & $35_{22,13}-34_{22,12}{ }^{\star}$ & 209795.133 & 11 & 467 & 453.5 & 45 & Blend with $\mathrm{C}_{2} \mathrm{H}_{3} \mathrm{CN}, v_{11}=1 / v_{15}=1$ \\
\hline 230 & $35_{19,16}-34_{19,15^{\star}}$ & 209804.307 & 10 & 426 & 528.7 & 45 & Strong $\mathrm{C}_{2} \mathrm{H}_{3} \mathrm{CN}, v_{11}=1$ and $\mathrm{CH}_{3} \mathrm{OCH}_{3}$ \\
\hline 232 & $15_{9,7}-14_{8,6}{ }^{\star}$ & 209810.239 & 12 & 184 & 81.0 & 45 & Strong $\mathrm{C}_{2} \mathrm{H}_{3} \mathrm{CN}, v_{11}=1$ and $\mathrm{CH}_{3} \mathrm{OCH}_{3}$ \\
\hline 234 & $35_{23,12}-34_{23,11}$ * & 209810.767 & 12 & 482 & 425.9 & 45 & Strong $\mathrm{C}_{2} \mathrm{H}_{3} \mathrm{CN}, v_{11}=1$ and $\mathrm{CH}_{3} \mathrm{OCH}_{3}$ \\
\hline 236 & $35_{18,17}-34_{18,16^{\star}}$ & 209831.596 & 10 & 413 & 551.4 & 45 & Blend with ${ }^{13} \mathrm{CH}_{3} \mathrm{CH}_{2} \mathrm{CN}$ \\
\hline 238 & $35_{24,11}-34_{24,10}$ * & 209833.824 & 13 & 498 & 397.2 & 45 & Blend with ${ }^{13} \mathrm{CH}_{3} \mathrm{CH}_{2} \mathrm{CN}$ \\
\hline 240 & $35_{25,10}-34_{25,9^{\star}}$ & 209863.488 & 15 & 515 & 367.2 & 45 & Strong $\mathrm{C}_{2} \mathrm{H}_{5} \mathrm{OH}, \mathrm{CH}_{3} \mathrm{OCHO}$, and ${ }^{13} \mathrm{CH}_{3} \mathrm{CH}_{2} \mathrm{CN}$ \\
\hline 242 & $35_{17,18}-34_{17,17^{\star}}$ & 209875.072 & 10 & 401 & 572.9 & 45 & Strong $\mathrm{CH}_{3} \mathrm{OCHO},{ }^{13} \mathrm{CH}_{3} \mathrm{CH}_{2} \mathrm{CN}$, and $\mathrm{C}_{2} \mathrm{H}_{5} \mathrm{CN}$ \\
\hline 244 & $35_{26,9}-34_{26,8}{ }^{\star}$ & 209899.094 & 17 & 532 & 336.0 & 45 & Blend with $\mathrm{C}_{2} \mathrm{H}_{3} \mathrm{CN}, v_{11}=1$ and ${ }^{13} \mathrm{CH}_{3} \mathrm{CH}_{2} \mathrm{CN}$ \\
\hline 246 & $35_{16,19}-34_{16,18}{ }^{\star}$ & 209938.416 & 10 & 390 & 593.0 & 45 & Strong $\mathrm{CH}_{3} \mathrm{OCHO}$ and $\mathrm{SO}_{2}$ \\
\hline 248 & $35_{27,8}-34_{27,7^{\star}}$ & 209940.094 & 19 & 550 & 303.6 & 45 & Strong $\mathrm{CH}_{3} \mathrm{OCHO}$ and $\mathrm{SO}_{2}$ \\
\hline 250 & $35_{15,20}-34_{15,19^{\star}}$ & 210026.654 & 10 & 380 & 612.0 & 45 & Strong $\mathrm{HC}_{3} \mathrm{~N}, v_{7}=1$ \\
\hline 252 & $37_{2,35}-36_{3,34}$ & 210086.448 & 6 & 322 & 251.9 & 45 & Blend with $\mathrm{C}_{2} \mathrm{H}_{3} \mathrm{CN}, v_{11}=2$ \\
\hline 253 & $37_{3,35}-36_{3,34}$ & 210090.303 & 6 & 322 & 779.0 & 45 & Blend with $\mathrm{C}_{2} \mathrm{H}_{3} \mathrm{CN}, v_{11}=2$ \\
\hline 254 & $35_{30,5}-34_{30,4}{ }^{\star}$ & 210091.291 & 29 & 607 & 198.9 & 45 & Blend with $\mathrm{C}_{2} \mathrm{H}_{3} \mathrm{CN}, v_{11}=2$ \\
\hline 256 & $37_{2,35}-36_{2,34}$ & 210092.485 & 6 & 322 & 779.0 & 45 & Blend with $\mathrm{C}_{2} \mathrm{H}_{3} \mathrm{CN}, v_{11}=2$ \\
\hline 257 & $37_{3,35}-36_{2,34}$ & 210096.339 & 6 & 322 & 251.9 & 45 & Blend with $\mathrm{C}_{2} \mathrm{H}_{3} \mathrm{CN}, v_{11}=2$ \\
\hline 258 & $35_{14,22}-34_{14,21}{ }^{\star}$ & 210146.825 & 9 & 370 & 629.7 & 64 & Blend with ${ }^{13} \mathrm{CH}_{3} \mathrm{CH}_{2} \mathrm{CN}$ \\
\hline 260 & $35_{31,4}-34_{31,3^{\star}}$ & 210150.008 & 35 & 628 & 161.6 & 64 & Blend with ${ }^{13} \mathrm{CH}_{3} \mathrm{CH}_{2} \mathrm{CN}$ \\
\hline 262 & $35_{13,23}-34_{13,22}{ }^{\star}$ & 210309.100 & 9 & 361 & 646.3 & 64 & Strong $\mathrm{HC}_{3} \mathrm{~N}, v_{6}=v_{7}=1$ and $\mathrm{C}_{2} \mathrm{H}_{5} \mathrm{CN}, v_{13}=1 / v_{21}=1$ \\
\hline 264 & $35_{5,31}-34_{4,30}$ & 210398.840 & 6 & 313 & 152.8 & 64 & Blend with $\mathrm{C}_{2} \mathrm{H}_{5} \mathrm{OH}$ and $\mathrm{CH}_{3} \mathrm{OCHO}$ \\
\hline 265 & $35_{12,24}-34_{12,23}{ }^{\star}$ & 210528.754 & 8 & 353 & 661.6 & 64 & Strong $\mathrm{HC}_{3} \mathrm{~N}, v_{7}=2$ and $\mathrm{C}_{2} \mathrm{H}_{5} \mathrm{OH}$ \\
\hline 267 & $20_{7,14}-19_{6,13}$ & 210694.282 & 12 & 199 & 69.5 & 37 & Blend with $\mathrm{C}_{2} \mathrm{H}_{3} \mathrm{CN}, v_{11}=2$ \\
\hline 268 & $35_{11,25}-34_{11,24}$ & 210829.766 & 7 & 345 & 675.7 & 37 & Blend with ${ }^{13} \mathrm{CH}_{3} \mathrm{OH}$ and $\mathrm{NH}_{2} \mathrm{CHO}$ \\
\hline 269 & $35_{11,24}-34_{11,23}$ & 210830.374 & 7 & 345 & 675.6 & 37 & Blend with ${ }^{13} \mathrm{CH}_{3} \mathrm{OH}$ and $\mathrm{NH}_{2} \mathrm{CHO}$ \\
\hline 270 & $20_{7,13}-19_{6,14}$ & 210837.991 & 12 & 199 & 69.5 & 37 & Blend with ${ }^{13} \mathrm{CH}_{3} \mathrm{OH}$ and $\mathrm{NH}_{2} \mathrm{CHO}$ \\
\hline 271 & $35_{6,30}-34_{6,29}$ & 210871.662 & 6 & 317 & 724.7 & 37 & Blend with $U$-line and $\mathrm{C}_{2} \mathrm{H}_{5} \mathrm{OH}$ \\
\hline 272 & $20_{5,15}-19_{3,16}$ & 210878.636 & 15 & 192 & 2.3 & 37 & Blend with $\mathrm{CH}_{3} \mathrm{OCHO},{ }^{13} \mathrm{CH}_{3} \mathrm{CH}_{2} \mathrm{CN}$, and $U$-line \\
\hline 273 & $35_{10,26}-34_{10,25}$ & 211249.809 & 7 & 338 & 688.5 & 33 & Strong $\mathrm{CH}_{3} \mathrm{OCHO}$ \\
\hline 274 & $35_{10,25}-34_{10,24}$ & 211259.223 & 7 & 338 & 688.5 & 33 & Strong $\mathrm{CH}_{3} \mathrm{OCHO}$ \\
\hline 275 & $38_{1,37}-37_{2,36} \star$ & 211401.390 & 50 & 325 & 296.5 & 33 & Blend with $\mathrm{C}_{2} \mathrm{H}_{5} \mathrm{CN}$ and $\mathrm{CH}_{3} \mathrm{CH}_{3} \mathrm{CO}, v_{t}=1$, uncertain baseline \\
\hline 277 & $38_{1,37}-37_{1,36}{ }^{\star}$ & 211401.444 & 9 & 325 & 805.2 & 33 & Blend with $\mathrm{C}_{2} \mathrm{H}_{5} \mathrm{CN}$ and $\mathrm{CH}_{3} \mathrm{CH}_{3} \mathrm{CO}, v_{t}=1$, uncertain baseline \\
\hline 279 & $36_{4,32}-35_{5,31}$ & 211527.010 & 50 & 323 & 162.7 & 47 & Blend with $\mathrm{C}_{2} \mathrm{H}_{3} \mathrm{CN}$ and $\mathrm{CH}_{3} \mathrm{OCHO}$ \\
\hline 280 & $35_{9,27}-34_{9,26}$ & 211828.060 & 50 & 332 & 700.1 & 47 & Blend with $U$-line, $\mathrm{C}_{2} \mathrm{H}_{5} \mathrm{OCHO}$, and $\mathrm{NH}_{2}{ }^{13} \mathrm{CHO}, v_{12}=1$ \\
\hline
\end{tabular}


Table 10. continued.

\begin{tabular}{|c|c|c|c|c|c|c|c|}
\hline$N^{a}$ & Transition $^{b}$ & $\begin{array}{c}\text { Frequency } \\
(\mathrm{MHz}) \\
(3) \\
\end{array}$ & $\begin{array}{c}\text { Unc. }^{c} \\
(\mathrm{kHz}) \\
(4) \\
\end{array}$ & $\begin{array}{l}E_{1}{ }^{d} \\
(\mathrm{~K}) \\
(5) \\
\end{array}$ & $\begin{array}{c}S \mu^{2} \\
\left(\mathrm{D}^{2}\right) \\
(6) \\
\end{array}$ & $\begin{array}{c}\sigma^{e} \\
(\mathrm{mK}) \\
(7) \\
\end{array}$ & Comments \\
\hline 281 & $35_{9,26}-34_{9,25}$ & 211938.010 & 50 & 332 & 700.1 & 36 & Strong $\mathrm{NH}_{2}{ }^{13} \mathrm{CHO}$ and $\mathrm{NH}_{2}{ }^{13} \mathrm{CHO}, v_{12}=1$ \\
\hline 282 & $13_{10,3}-129,4^{\star}$ & 211955.981 & 12 & 182 & 87.6 & 36 & Strong $\mathrm{NH}_{2}{ }^{13} \mathrm{CHO}, v_{12}=1$ and $\mathrm{NH}_{2}{ }^{13} \mathrm{CHO}$ \\
\hline 284 & $34_{6,28}-33_{6,27}$ & 212120.060 & 50 & 309 & 708.0 & 36 & Strong $\mathrm{CH}_{3} \mathrm{CHO}$ and $\mathrm{NH}_{2}{ }^{13} \mathrm{CHO}$ \\
\hline 285 & $35_{8,28}-34_{8,27}$ & 212458.290 & 50 & 326 & 710.4 & 99 & Blend with $\mathrm{C}_{2} \mathrm{H}_{3} \mathrm{CN}$ and $\mathrm{C}_{2} \mathrm{H}_{5} \mathrm{OH}$ \\
\hline 286 & $35_{7,29}-34_{7,28}$ & 212483.750 & 50 & 321 & 719.0 & 99 & Blend with $\mathrm{CH}_{3} \mathrm{OCHO}, \mathrm{a}-\mathrm{C}_{3} \mathrm{H}_{7} \mathrm{CN}$, and ${ }^{13} \mathrm{CH}_{3} \mathrm{OH}, v_{t}=1$ \\
\hline 287 & $39_{1,39}-38_{1,38}$ * & 212743.017 & 15 & 328 & 832.1 & 99 & Strong $\mathrm{CH}_{3} \mathrm{OCH}_{3}$ and $\mathrm{C}_{2} \mathrm{H}_{5} \mathrm{OH}$ \\
\hline 289 & $39_{1,39}-38_{0,38}$ * & 212743.018 & 15 & 328 & 339.8 & 99 & Strong $\mathrm{CH}_{3} \mathrm{OCH}_{3}$ and $\mathrm{C}_{2} \mathrm{H}_{5} \mathrm{OH}$ \\
\hline 291 & $36_{5,32}-35_{5,31}$ & 212972.357 & 6 & 323 & 749.7 & 99 & Blend with ${ }^{13} \mathrm{CH}_{3} \mathrm{CH}_{2} \mathrm{CN}$ and ${ }^{34} \mathrm{SO}_{2}$ \\
\hline 292 & $35_{8,27}-34_{8,26}$ & 213353.646 & 6 & 326 & 710.6 & 48 & Blend with ${ }^{13} \mathrm{CH}_{2} \mathrm{CHCN}, \mathrm{C}_{2} \mathrm{H}_{5} \mathrm{CN}$, and $U$-line \\
\hline 293 & $18_{8,11}-17_{7,10}$ & 213473.443 & 12 & 193 & 76.1 & 48 & Blend with $\mathrm{C}_{2} \mathrm{H}_{5}{ }^{13} \mathrm{CN}$ \\
\hline 294 & $50_{11,40}-50_{9,41}$ & 213474.581 & 160 & 532 & 6.1 & 48 & Blend with $\mathrm{C}_{2} \mathrm{H}_{5}{ }^{13} \mathrm{CN}$ \\
\hline 295 & $18_{8,10}-17_{7,11}$ & 213474.845 & 12 & 193 & 76.1 & 48 & Blend with $\mathrm{C}_{2} \mathrm{H}_{5}{ }^{13} \mathrm{CN}$ \\
\hline 296 & $36_{4,32}-35_{4,31}$ & 213568.985 & 6 & 323 & 749.9 & 48 & Blend with $\mathrm{C}_{2} \mathrm{H}_{3} \mathrm{CN}, v_{11}=1 / v_{15}=1$ and ${ }^{13} \mathrm{CH}_{2} \mathrm{CHCN}$ \\
\hline 297 & $11_{11,0}-10_{10,1}{ }^{\star}$ & 214056.089 & 12 & 182 & 95.9 & 48 & Strong ${ }^{13} \mathrm{CH}_{3} \mathrm{CN}, \mathrm{C}_{2} \mathrm{H}_{5}{ }^{13} \mathrm{CN}$, and $\mathrm{CH}_{3}{ }^{13} \mathrm{CH}_{2} \mathrm{CN}$ \\
\hline 299 & $37_{3,34}-36_{4,33}$ & 214147.290 & 50 & 328 & 214.4 & 75 & Strong $\mathrm{C}_{2} \mathrm{H}_{5}{ }^{13} \mathrm{CN}$ and $\mathrm{CH}_{3}{ }^{13} \mathrm{CH}_{2} \mathrm{CN}$ \\
\hline 300 & $37_{4,34}-36_{4,33}$ & 214225.810 & 50 & 328 & 774.6 & 75 & Strong $\mathrm{CH}_{2} \mathrm{NH},{ }^{13} \mathrm{CH}_{3} \mathrm{CN}$, and $\mathrm{C}_{2} \mathrm{H}_{5} \mathrm{CN}$ \\
\hline 301 & $37_{3,34}-36_{3,33}$ & 214265.300 & 50 & 328 & 774.6 & 75 & Strong $\mathrm{C}_{2} \mathrm{H}_{5} \mathrm{CN}$ and $\mathrm{C}_{2} \mathrm{H}_{5} \mathrm{OH}$ \\
\hline 302 & $37_{4,34}-36_{3,33}$ & 214343.817 & 6 & 328 & 214.4 & 75 & Strong ${ }^{13} \mathrm{CH}_{3} \mathrm{CN}$ and $\mathrm{C}_{2} \mathrm{H}_{5}{ }^{13} \mathrm{CN}$ \\
\hline 303 & $35_{5,30}-34_{5,29}$ & 214468.257 & 6 & 316 & 728.6 & 75 & Blend with $\mathrm{CH}_{3} \mathrm{OCH}_{3}$ \\
\hline 304 & $51_{16,35}-51_{15,36}$ & 214470.968 & 53 & 591 & 243.9 & 75 & Blend with $\mathrm{CH}_{3} \mathrm{OCH}_{3}$ \\
\hline 305 & $51_{16,36}-51_{15,37}$ & 214471.118 & 53 & 591 & 243.9 & 75 & Blend with $\mathrm{CH}_{3} \mathrm{OCH}_{3}$ \\
\hline 306 & $36_{5,32}-35_{4,31}$ & 215014.344 & 6 & 323 & 163.1 & 74 & Blend with ${ }^{13} \mathrm{CH}_{3} \mathrm{CN}, v_{8}=1$ \\
\hline 307 & $38_{2,36}-37_{3,35}$ & 215469.850 & 50 & 332 & 261.1 & 74 & Strong $\mathrm{C}_{2} \mathrm{H}_{5} \mathrm{CN}, v_{13}=1 / v_{21}=1$ \\
\hline 308 & $38_{3,36}-37_{3,35}$ & 215472.342 & 7 & 332 & 800.3 & 74 & Strong $\mathrm{C}_{2} \mathrm{H}_{5} \mathrm{CN}, v_{13}=1 / v_{21}=1$ \\
\hline 309 & $38_{2,36}-37_{2,35}$ & 215473.742 & 7 & 332 & 800.3 & 74 & Strong $\mathrm{C}_{2} \mathrm{H}_{5} \mathrm{CN}, v_{13}=1 / v_{21}=1$ \\
\hline 310 & $38_{3,36}-37_{2,35}$ & 215476.230 & 50 & 332 & 261.1 & 74 & Strong $\mathrm{C}_{2} \mathrm{H}_{5} \mathrm{CN}, v_{13}=1 / v_{21}=1$ \\
\hline 311 & $21_{5,16}-20_{3,17}$ & 215480.099 & 15 & 198 & 2.7 & 74 & Strong $\mathrm{C}_{2} \mathrm{H}_{5} \mathrm{CN}, v_{13}=1 / v_{21}=1$ \\
\hline 312 & $16_{9,8}-15_{8,7^{\star}}^{\star}$ & 215778.326 & 12 & 189 & 82.0 & 74 & Blend with $\mathrm{C}_{2} \mathrm{H}_{5} \mathrm{CN}, v_{20}=1$ \\
\hline 314 & 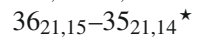 & 215779.220 & 50 & 463 & 508.7 & 74 & Blend with $\mathrm{C}_{2} \mathrm{H}_{5} \mathrm{CN}, v_{20}=1$ \\
\hline 316 & $36_{22,14}-35_{22,13}{ }^{\star}$ & 215783.850 & 50 & 477 & 483.1 & 74 & Blend with $\mathrm{C}_{2} \mathrm{H}_{5} \mathrm{CN}, v_{20}=1$ \\
\hline 318 & $36_{20,16}-35_{20,15}{ }^{\star}$ & 215784.950 & 50 & 449 & 533.2 & 74 & Blend with $\mathrm{C}_{2} \mathrm{H}_{5} \mathrm{CN}, v_{20}=1$ \\
\hline 320 & $36_{23,13}-35_{23,12}$ * & 215797.530 & 50 & 492 & 456.3 & 74 & Blend with $\mathrm{C}_{2} \mathrm{H}_{5} \mathrm{CN}, v_{13}=1 / v_{21}=1$ \\
\hline 322 & $36_{19,17}-35_{19,16^{\star}}$ & 215802.872 & 10 & 436 & 556.3 & 74 & Blend with $\mathrm{C}_{2} \mathrm{H}_{5} \mathrm{CN}, v_{13}=1 / v_{21}=1$ \\
\hline 324 & $36_{24,12}-35_{24,11}$ * & 215819.220 & 50 & 508 & 428.4 & 55 & Strong $\mathrm{C}_{2} \mathrm{H}_{5} \mathrm{CN}, v_{13}=1 / v_{21}=1$ \\
\hline 326 & $36_{18,18}-35_{18,17^{\star}}$ & 215835.300 & 50 & 423 & 578.4 & 55 & Strong $\mathrm{C}_{2} \mathrm{H}_{5} \mathrm{CN}, v_{13}=1 / v_{21}=1$ and ${ }^{34} \mathrm{SO}$ \\
\hline 328 & $36_{25,11}-35_{25,10}$ * & 215847.810 & 50 & 525 & 399.2 & 55 & Strong ${ }^{34} \mathrm{SO}$ \\
\hline 330 & $36_{26,10}-35_{26,9^{\star}}$ & 215882.850 & 50 & 542 & 368.9 & 55 & Strong ${ }^{13} \mathrm{CH}_{3} \mathrm{OH}$ \\
\hline 332 & $36_{17,19}-35_{17,18^{\star}}$ & 215885.180 & 50 & 411 & 599.1 & 55 & Strong ${ }^{13} \mathrm{CH}_{3} \mathrm{OH}$ and $\mathrm{C}_{2} \mathrm{H}_{5} \mathrm{OH}$ \\
\hline 334 & $36_{27,9}-35_{27,8}$ * & 215923.600 & 50 & 560 & 337.4 & 55 & Blend with $\mathrm{C}_{2} \mathrm{H}_{5} \mathrm{OH}, \mathrm{CH}_{3} \mathrm{COOH}$, and $U$-line \\
\hline 336 & $36_{16,20}-35_{16,19}{ }^{\star}$ & 215956.570 & 50 & 400 & 618.7 & 55 & Strong $\mathrm{C}_{2} \mathrm{H}_{5} \mathrm{CN}$ \\
\hline 338 & 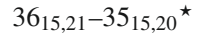 & 216055.006 & 10 & 390 & 637.2 & 55 & Strong $\mathrm{C}_{2} \mathrm{H}_{5} \mathrm{CN}, v_{13}=1 / v_{21}=1$ \\
\hline 340 & $36_{14,22}-35_{14,21}{ }^{\star}$ & 216188.170 & 50 & 380 & 654.5 & 55 & Blend with $\mathrm{C}_{2} \mathrm{H}_{5} \mathrm{CN}$ \\
\hline 342 & $36_{32,4}-35_{32,3}{ }^{\star}$ & 216198.450 & 50 & 659 & 161.8 & 55 & Blend with $\mathrm{CH}_{3} \mathrm{OCHO}$ \\
\hline 344 & $36_{13,23}-35_{13,22}{ }^{\star}$ & 216367.230 & 50 & 371 & 670.6 & 55 & Strong $\mathrm{CH}_{3} \mathrm{OCHO}$ and ${ }^{13} \mathrm{CH}_{3} \mathrm{OH}$ \\
\hline 346 & $21_{7,15}-20_{6,14}$ & 216386.644 & 12 & 205 & 70.1 & 55 & Blend with $U$-line \\
\hline 347 & $35_{7,28}-34_{7,27}$ & 216489.383 & 6 & 322 & 720.8 & 55 & Blend with $\mathrm{C}_{2} \mathrm{H}_{5} \mathrm{CN}, v_{13}=1 / v_{21}=1$ and $U$-line \\
\hline 348 & $36_{6,31}-35_{6,30}$ & 216511.289 & 7 & 327 & 746.2 & 55 & Strong $\mathrm{CH}_{3} \mathrm{CH}_{3} \mathrm{CO}, v_{t}=1$ and $\mathrm{C}_{2} \mathrm{H}_{5} \mathrm{CN}, v_{13}=1 / v_{21}=1$ \\
\hline 349 & $36_{12,24}-35_{12,23}$ * & 216609.170 & 50 & 363 & 685.5 & 55 & Blend with $\mathrm{CH}_{3} \mathrm{COOH}$ \\
\hline 351 & $21_{7,14}-20_{6,15}$ & 216649.055 & 12 & 205 & 70.1 & 55 & Strong $\mathrm{SO}_{2}$ \\
\hline 352 & $39_{1,38}-38_{2,37}$ * & 216786.652 & 10 & 335 & 305.7 & 50 & Blend with $U$-line and $\mathrm{CH}_{3} \mathrm{CH}_{3} \mathrm{CO}, v_{t}=1$ \\
\hline 354 & $39_{1,38}-38_{1,37^{\star}}$ & 216786.721 & 10 & 335 & 826.7 & 50 & Blend with $U$-line and $\mathrm{CH}_{3} \mathrm{CH}_{3} \mathrm{CO}, v_{t}=1$ \\
\hline 356 & $42_{16,26}-42_{15,27} \star$ & 216853.893 & 37 & 469 & 186.2 & 50 & Strong $\mathrm{C}_{2} \mathrm{H}_{5} \mathrm{CN}, v_{13}=1 / v_{21}=1$ \\
\hline 358 & $36_{11,26}-35_{11,25}$ & 216940.590 & 50 & 355 & 699.1 & 50 & Strong $\mathrm{C}_{2} \mathrm{H}_{3} \mathrm{CN}$ and $\mathrm{CH}_{3} \mathrm{OH}$ \\
\hline 359 & $36_{11,25}-35_{11,24}$ & 216941.740 & 50 & 355 & 699.1 & 50 & Strong $\mathrm{C}_{2} \mathrm{H}_{3} \mathrm{CN}$ and $\mathrm{CH}_{3} \mathrm{OH}$ \\
\hline 360 & $41_{16,25}-41_{15,26}{ }^{\star}$ & 217028.389 & 36 & 456 & 179.9 & 50 & Blend with $\mathrm{CH}_{3} \mathrm{CH}_{3} \mathrm{CO}$ and ${ }^{13} \mathrm{CN}$ in absorption \\
\hline 362 & $40_{16,24}-40_{15,25}^{\star}$ & 217188.266 & 36 & 445 & 173.6 & 50 & Strong $\mathrm{CH}_{3} \mathrm{OCH}_{3}$ \\
\hline 364 & $39_{16,23}-39_{15,24}$ * & 217334.340 & 35 & 433 & 167.4 & 50 & Blend with $\mathrm{CH}_{2}{ }^{13} \mathrm{CHCN}$ \\
\hline 366 & $37_{4,33}-36_{5,32}$ & 217368.200 & 8 & 333 & 173.0 & 50 & Blend with ${ }^{13} \mathrm{CN}$ in absorption, $\mathrm{C}_{2} \mathrm{H}_{5}{ }^{13} \mathrm{CN}$, and $U$-line? \\
\hline 367 & $36_{10,27}-35_{10,26}$ & 217402.380 & 50 & 348 & 711.7 & 50 & Strong $\mathrm{HC}^{13} \mathrm{CCN}$ and ${ }^{13} \mathrm{CH}_{3} \mathrm{OH}$ \\
\hline 368 & $36_{10,26}-35_{10,25}$ & 217418.120 & 50 & 348 & 711.6 & 50 & Strong $\mathrm{HCC}^{13} \mathrm{CN}$ \\
\hline 369 & $38_{16,22}-38_{15,23}{ }^{\star}$ & 217467.396 & 35 & 422 & 161.1 & 50 & Blend with ${ }^{13} \mathrm{CN}$ in absorption \\
\hline 371 & $37_{16,21}-37_{15,22}$ * & 217588.187 & 34 & 411 & 154.8 & 50 & Blend with $\left(\mathrm{CH}_{2} \mathrm{OH}\right)_{2}$ and $U$-line \\
\hline 373 & $36_{16,20}-36_{15,21}$ * & 217697.439 & 34 & 400 & 148.6 & 50 & Blend with $\mathrm{CH}_{2} \mathrm{CH}^{13} \mathrm{CN}$ and $U$-line \\
\hline
\end{tabular}


A. Belloche et al.: Detection and chemical modeling of ethyl formate and $n$-propyl cyanide in Sgr B2(N), Online Material $p 30$

Table 10. continued.

\begin{tabular}{|c|c|c|c|c|c|c|c|}
\hline$N^{a}$ & $\begin{array}{c}\text { Transition }^{b} \\
\text { (2) }\end{array}$ & $\begin{array}{c}\text { Frequency } \\
\text { (MHz) } \\
\text { (3) }\end{array}$ & $\begin{array}{c}\text { Unc. }^{c} \\
(\mathrm{kHz}) \\
(4)\end{array}$ & $\begin{array}{l}E_{1}^{d} \\
(\mathrm{~K}) \\
(5)\end{array}$ & $\begin{array}{l}S \mu^{2} \\
\left(\mathrm{D}^{2}\right) \\
(6)\end{array}$ & $\begin{array}{c}\sigma^{e} \\
(\mathrm{mK}) \\
(7)\end{array}$ & Comments \\
\hline 375 & $39_{8,31}-39_{5,34}$ & 219371.585 & 37 & 370 & 11.6 & 92 & Blend with $\mathrm{C}_{2} \mathrm{H}_{3} \mathrm{CN}, v_{11}=1 / v_{15}=1$ \\
\hline 376 & $198,12-18_{7,11}$ & 219376.897 & 12 & 198 & 77.1 & 92 & Blend with $\mathrm{C}_{2} \mathrm{H}_{3} \mathrm{CN}, v_{11}=1 / v_{15}=1$ and $\mathrm{C}_{2} \mathrm{H}_{5} \mathrm{OH}$ \\
\hline 377 & $19_{8,11}-18_{7,12}$ & 219380.063 & 12 & 198 & 77.1 & 92 & Blend with $\mathrm{C}_{2} \mathrm{H}_{3} \mathrm{CN}, v_{11}=1 / v_{15}=1$ and $\mathrm{C}_{2} \mathrm{H}_{5} \mathrm{OH}$ \\
\hline 378 & $38_{3,35}-374,34$ & 219549.670 & 50 & 338 & 223.9 & 92 & Strong $\mathrm{C}^{18} \mathrm{O}$ \\
\hline 379 & $36_{5,31}-35_{5,30}$ & 219565.844 & 7 & 327 & 748.9 & 92 & Strong $\mathrm{C}^{18} \mathrm{O}$ \\
\hline 380 & $38_{4,35}-37_{4,34}$ & 219601.640 & 50 & 338 & 796.0 & 92 & Blend with $\mathrm{CH}_{3} \mathrm{CN}, v_{4}=1$ and $\mathrm{NH}_{2} \mathrm{CN}$ \\
\hline 381 & $38_{3,35}-37_{3,34}$ & 219628.210 & 50 & 338 & 796.0 & 92 & Strong $\mathrm{C}_{2} \mathrm{H}_{5} \mathrm{CN}, v_{20}=1$ and $\mathrm{CH}_{3} \mathrm{CN}, v_{4}=1$ \\
\hline 382 & $58_{2,56}-58_{1,57^{\star}}$ & 219628.923 & 369 & 593 & 66.8 & 92 & Strong $\mathrm{C}_{2} \mathrm{H}_{5} \mathrm{CN}, v_{20}=1$ and $\mathrm{CH}_{3} \mathrm{CN}, v_{4}=1$ \\
\hline 384 & $58_{3,56}-58_{2,57} \star$ & 219628.923 & 369 & 593 & 66.8 & 92 & Strong $\mathrm{C}_{2} \mathrm{H}_{5} \mathrm{CN}, v_{20}=1$ and $\mathrm{CH}_{3} \mathrm{CN}, v_{4}=1$ \\
\hline 386 & $63_{11,53}-62_{12,50}$ & 219678.339 & 347 & 748 & 53.1 & 92 & Strong $\mathrm{HC}_{3} \mathrm{~N}, v_{7}=2$ \\
\hline 387 & $38_{4,35}-37_{3,34}$ & 219680.190 & 7 & 338 & 223.9 & 92 & Strong $\mathrm{HC}_{3} \mathrm{~N}, v_{7}=2$ \\
\hline 388 & $13_{11,2}-12_{10,3^{\star}}$ & 226038.817 & 12 & 189 & 96.1 & 278 & Blend with $\mathrm{c}-\mathrm{C}_{2} \mathrm{H}_{4} \mathrm{O}$ \\
\hline 390 & $40_{2,38}-39_{3,37}$ & 226232.383 & 9 & 353 & 279.7 & 278 & Noisy, blend with $U$-line? \\
\hline 391 & $40_{3,38}-39_{3,37}$ & 226233.371 & 9 & 353 & 843.2 & 278 & Noisy, blend with $U$-line? \\
\hline 392 & $40_{2,38}-39_{2,37}$ & 226233.942 & 9 & 353 & 843.2 & 278 & Noisy, blend with $U$-line? \\
\hline 393 & $40_{3,38}-39_{2,37}$ & 226234.930 & 9 & 353 & 279.6 & 278 & Noisy, blend with $U$-line? \\
\hline 394 & $59_{17,42}-59_{16,43}$ & 226530.302 & 99 & 729 & 290.8 & 278 & Blend with $\mathrm{C}_{2} \mathrm{H}_{5} \mathrm{CN}$ \\
\hline 395 & $59_{17,43}-59_{16,44}$ & 226530.999 & 99 & 729 & 290.8 & 278 & Blend with $\mathrm{C}_{2} \mathrm{H}_{5} \mathrm{CN}$ \\
\hline 396 & $37_{8,29}-36_{8,28}$ & 226531.433 & 7 & 347 & 755.6 & 278 & Blend with $\mathrm{C}_{2} \mathrm{H}_{5} \mathrm{CN}$ \\
\hline 397 & $62_{3,59}-62_{3,60}^{\star}$ & 226531.914 & 447 & 668 & 15.8 & 278 & Blend with $\mathrm{C}_{2} \mathrm{H}_{5} \mathrm{CN}$ \\
\hline 399 & $62_{4,59}-62_{3,60}{ }^{\star}$ & 226531.915 & 447 & 668 & 100.4 & 278 & Blend with $\mathrm{C}_{2} \mathrm{H}_{5} \mathrm{CN}$ \\
\hline 401 & $23_{7,17}-22_{6,16}$ & 227460.224 & 12 & 218 & 70.9 & 85 & Blend with $\mathrm{CH}_{3} \mathrm{COOH}, \mathrm{CH}_{2}(\mathrm{OH}) \mathrm{CHO}$, and $\mathrm{CH}_{2} \mathrm{CH}^{13} \mathrm{CN}$ \\
\hline 402 & $41_{1,40}-40_{2,39}{ }^{\star}$ & 227553.664 & 14 & 357 & 324.0 & 85 & Strong $\mathrm{C}_{2} \mathrm{H}_{5} \mathrm{CN}$ and $\mathrm{CH}_{3} \mathrm{OCHO}$ \\
\hline 404 & $41_{1,40}-40_{1,39}{ }^{\star}$ & 227553.690 & 14 & 357 & 869.6 & 85 & Strong $\mathrm{C}_{2} \mathrm{H}_{5} \mathrm{CN}$ and $\mathrm{CH}_{3} \mathrm{OCHO}$ \\
\hline 406 & $60_{2,58}-60_{1,59}{ }^{\star}$ & 227625.680 & 458 & 624 & 66.9 & 85 & Strong $\mathrm{C}_{2} \mathrm{H}_{5} \mathrm{CN}, v_{13}=1 / v_{21}=1$ \\
\hline 408 & $60_{2,58}-60_{2,59}{ }^{\star}$ & 227625.680 & 458 & 624 & 10.9 & 85 & Strong $\mathrm{C}_{2} \mathrm{H}_{5} \mathrm{CN}, v_{13}=1 / v_{21}=1$ \\
\hline 410 & $38_{6,33}-37_{6,32}$ & 227639.839 & 9 & 348 & 788.8 & 85 & Strong $\mathrm{C}_{2} \mathrm{H}_{5} \mathrm{CN}, v_{13}=1 / v_{21}=1$ and $\mathrm{CH}_{3} \mathrm{OCHO}$ \\
\hline 411 & $189,10^{-17} 8,9^{\star}$ & 227689.981 & 12 & 198 & 84.0 & 85 & Blend with $U$-line \\
\hline 413 & $23_{5,18}-22_{3,19}$ & 227691.505 & 14 & 210 & 3.5 & 85 & Blend with $U$-line \\
\hline 414 & $38_{22,16}-37_{22,15}{ }^{\star}$ & 227759.604 & 11 & 498 & 541.1 & 85 & Blend with $\mathrm{H}_{2}{ }^{13} \mathrm{CS}$ and $\mathrm{C}_{2} \mathrm{H}_{5} \mathrm{OH}$ \\
\hline 416 & $38_{21,17}-37_{21,16}{ }^{\star}$ & 227760.667 & 11 & 484 & 565.4 & 85 & Blend with $\mathrm{H}_{2}{ }^{13} \mathrm{CS}$ and $\mathrm{C}_{2} \mathrm{H}_{5} \mathrm{OH}$ \\
\hline 418 & $38_{23,15}-37_{23,14}{ }^{\star}$ & 227768.859 & 12 & 513 & 515.7 & 85 & Strong $\mathrm{C}_{2} \mathrm{H}_{5} \mathrm{CN}$ \\
\hline 420 & $38_{20,18}-37_{20,17^{\star}}$ & 227773.682 & 11 & 470 & 588.4 & 85 & Strong $\mathrm{C}_{2} \mathrm{H}_{5} \mathrm{CN}$ \\
\hline 422 & $38_{24,14}-37_{24,13}{ }^{\star}$ & 227787.136 & 12 & 529 & 489.3 & 85 & Strong $\mathrm{C}_{2} \mathrm{H}_{5} \mathrm{CN}$ and $\mathrm{HC}_{3} \mathrm{~N}, v_{6}=1$ \\
\hline 424 & $38_{19,19}-37_{19,18^{\star}}$ & 227800.733 & 11 & 457 & 610.4 & 85 & Strong ${ }^{13} \mathrm{CH}_{3} \mathrm{CH}_{2} \mathrm{CN}$ and $\mathrm{CH}_{3} \mathrm{OH}$ \\
\hline 426 & $38_{25,13}-37_{25,12^{\star}}$ & 227813.392 & 14 & 546 & 461.7 & 85 & Strong $\mathrm{CH}_{3} \mathrm{OH}$ \\
\hline 428 & $56_{17,39}-56_{16,40}$ & 227818.866 & 80 & 679 & 270.6 & 85 & Strong $\mathrm{CH}_{3} \mathrm{OH}$ \\
\hline 429 & $56_{17,40}-56_{16,41}$ & 227819.010 & 80 & 679 & 270.6 & 85 & Strong $\mathrm{CH}_{3} \mathrm{OH}$ \\
\hline 430 & $38_{18,20}-37_{18,19}{ }^{\star}$ & 227844.521 & 11 & 444 & 631.3 & 85 & Blend with $\mathrm{CH}_{3} \mathrm{CH}_{3} \mathrm{CO}, \mathrm{HC}^{13} \mathrm{CCN}, v_{7}=2$, and $\mathrm{HCC}^{13} \mathrm{CN}, v_{7}=2$ \\
\hline 432 & $38_{26,12}-37_{26,11}$ * & 227846.781 & 15 & 563 & 432.9 & 85 & Blend with $\mathrm{CH}_{3} \mathrm{CH}_{3} \mathrm{CO}, \mathrm{HC}^{13} \mathrm{CCN}, v_{7}=2$, and $\mathrm{HCC}^{13} \mathrm{CN}, v_{7}=2$ \\
\hline 434 & $38_{27,11}-37_{27,10}{ }^{\star}$ & 227886.609 & 17 & 581 & 403.0 & 85 & Strong $\mathrm{C}_{2} \mathrm{H}_{5} \mathrm{OH}$ \\
\hline 436 & $38_{17,21}-37_{17,20}$ * & 227908.603 & 11 & 432 & 651.0 & 85 & Strong $\mathrm{CH}_{3}{ }^{13} \mathrm{CH}_{2} \mathrm{CN}$ and $\mathrm{C}_{2} \mathrm{H}_{3} \mathrm{CN}$ \\
\hline 438 & $44_{17,27}-44_{16,28}{ }^{\star}$ & 231002.504 & 54 & 504 & 193.5 & 183 & Noisy \\
\hline 440 & $21_{8,14}-20_{7,13}$ & 231114.753 & 12 & 210 & 78.8 & 183 & Blend with $\mathrm{C}_{2} \mathrm{H}_{3} \mathrm{CN}, v_{11}=1, \mathrm{CH}_{3} \mathrm{CHO}$, and $U$-line \\
\hline 441 & $21_{8,13}-200_{7,14}$ & 231128.746 & 12 & 210 & 78.8 & 183 & Blend with $U$-line and $\mathrm{H}_{2}{ }^{13} \mathrm{CS}$ \\
\hline 442 & $43_{17,26}-43_{16,27^{\star}}$ & 231159.401 & 54 & 491 & 187.2 & 183 & Strong ${ }^{13} \mathrm{CH}_{2} \mathrm{CHCN}$ \\
\hline 444 & $42_{17,25}-42_{16,26}{ }^{\star}$ & 231303.368 & 53 & 479 & 180.9 & 40 & Strong ${ }^{13} \mathrm{CH}_{3} \mathrm{CH}_{2} \mathrm{CN}$ and $\mathrm{C}_{2} \mathrm{H}_{5} \mathrm{CN}$ \\
\hline 446 & $41_{17,24}-41_{16,25}{ }^{\star}$ & 231435.094 & 53 & 467 & 174.7 & 40 & Blend with ${ }^{13} \mathrm{CH}_{2} \mathrm{CHCN}$ \\
\hline 448 & $40_{17,23}-40_{16,24}$ * & 231555.240 & 53 & 455 & 168.4 & 40 & Blend with $\mathrm{C}_{2} \mathrm{H}_{5} \mathrm{OH}$ \\
\hline 450 & $41_{2,39}-40_{3,38}$ & 231611.663 & 10 & 364 & 288.9 & 40 & Blend with $\mathrm{HNCO}, v_{5}=1, \mathrm{CH}_{3} \mathrm{OCHO},{ }^{13} \mathrm{CH}_{3} \mathrm{CN}$, and $U$-line \\
\hline 451 & $41_{3,39}-40_{3,38}$ & 231612.288 & 10 & 364 & 864.4 & 40 & Blend with $\mathrm{HNCO}, v_{5}=1, \mathrm{CH}_{3} \mathrm{OCHO},{ }^{13} \mathrm{CH}_{3} \mathrm{CN}$, and $U$-line \\
\hline 452 & $41_{2,39}-40_{2,38}$ & 231612.651 & 10 & 364 & 864.6 & 40 & Blend with $\mathrm{HNCO}, v_{5}=1, \mathrm{CH}_{3} \mathrm{OCHO},{ }^{13} \mathrm{CH}_{3} \mathrm{CN}$, and $U$-line \\
\hline 453 & $41_{3,39}-40_{2,38}$ & 231613.276 & 10 & 364 & 288.9 & 40 & Blend with $\mathrm{HNCO}, v_{5}=1, \mathrm{CH}_{3} \mathrm{OCHO},{ }^{13} \mathrm{CH}_{3} \mathrm{CN}$, and $U$-line \\
\hline 454 & $61_{2,59}-61_{1,60}^{\star}$ & 231619.484 & 508 & 640 & 66.9 & 40 & Blend with $\mathrm{CH}_{3} \mathrm{OCHO},{ }^{13} \mathrm{CH}_{3} \mathrm{CN}$, and $U$-line \\
\hline 456 & $61_{3,59}-61_{2,60}^{\star}$ & 231619.484 & 508 & 640 & 66.9 & 40 & Blend with $\mathrm{CH}_{3} \mathrm{OCHO},{ }^{13} \mathrm{CH}_{3} \mathrm{CN}$, and $U$-line \\
\hline 458 & $58_{11,47}-57_{12,46}$ & 231619.899 & 325 & 659 & 71.0 & 40 & Blend with $\mathrm{CH}_{3} \mathrm{OCHO},{ }^{13} \mathrm{CH}_{3} \mathrm{CN}$, and $U$-line \\
\hline 459 & $39_{17,22}-39_{16,23}{ }^{\star}$ & 231664.446 & 53 & 444 & 162.2 & 40 & Blend with ${ }^{13} \mathrm{CH}_{3} \mathrm{CH}_{2} \mathrm{CN}$ and $\mathrm{C}_{2} \mathrm{H}_{5} \mathrm{OH}$ \\
\hline 461 & $37_{6,32}-36_{5,31}$ & 232675.249 & 9 & 337 & 125.2 & 19 & Blend with $\mathrm{CH}_{3} \mathrm{CH}_{3} \mathrm{CO}, v_{t}=1$ and $U$-line \\
\hline 462 & $24_{7,18}-23_{6,17}$ & 232766.448 & 12 & 224 & 71.1 & 19 & Blend with ${ }^{13} \mathrm{CH}_{3} \mathrm{CN}, v_{8}=1$ and $\mathrm{CH}_{3} \mathrm{CH}_{3} \mathrm{CO}$ \\
\hline 463 & $40_{5,36}-39_{4,35}$ & 235023.563 & 9 & 365 & 203.0 & 131 & Blend with $\mathrm{CH}_{3} \mathrm{OCHO}$ \\
\hline 464 & $39_{11,29}-38_{11,28}$ & 235323.229 & 8 & 387 & 768.9 & 131 & Blend with $\mathrm{CH}_{3} \mathrm{CHO}$ and $U$-line \\
\hline 465 & $23_{4,19}-22_{3,20}$ & 235326.210 & 10 & 208 & 11.1 & 131 & Blend with $\mathrm{CH}_{3} \mathrm{CHO}$ and $U$-line \\
\hline 466 & $39_{11,28}-38_{11,27}$ & 235328.787 & 8 & 387 & 769.0 & 131 & Blend with $\mathrm{CH}_{3} \mathrm{CHO}$ and $U$-line \\
\hline
\end{tabular}


Table 10. continued.

\begin{tabular}{|c|c|c|c|c|c|c|c|}
\hline$N^{a}$ & Transition $^{b}$ & $\begin{array}{c}\text { Frequency } \\
\text { (MHz) } \\
\text { (3) }\end{array}$ & $\begin{array}{c}\text { Unc. }^{c} \\
(\mathrm{kHz}) \\
(4)\end{array}$ & $\begin{array}{l}E_{1}^{d} \\
(\mathrm{~K}) \\
(5)\end{array}$ & $\begin{array}{l}S \mu^{2} \\
\left(\mathrm{D}^{2}\right) \\
(6)\end{array}$ & $\begin{array}{c}\sigma^{e} \\
(\mathrm{mK}) \\
(7)\end{array}$ & Comments \\
\hline 467 & $40_{5,35}-39_{6,34}$ & 235342.332 & 14 & 370 & 156.8 & 131 & Blend with $U$-line and $\mathrm{CH}_{3} \mathrm{CH}_{3} \mathrm{CO}$ \\
\hline 468 & $62_{2,60}-62_{1,61}^{\star}$ & 235610.226 & 562 & 656 & 67.0 & 131 & $\begin{array}{l}\text { Blend with } \mathrm{CH}_{3} \mathrm{CH}_{3} \mathrm{CO},{ }^{13} \mathrm{CH}_{3} \mathrm{OH}, v_{t}=1 \text {, and } \\
\mathrm{C}_{2} \mathrm{H}_{5} \mathrm{CN}, v_{13}=1 / v_{21}=1\end{array}$ \\
\hline 470 & $62_{2,60}-62_{2,61}{ }^{\star}$ & 235610.226 & 562 & 656 & 10.9 & 131 & $\begin{array}{l}\text { Blend with } \mathrm{CH}_{3} \mathrm{CH}_{3} \mathrm{CO},{ }^{13} \mathrm{CH}_{3} \mathrm{OH}, v_{t}=1 \text {, and } \\
\mathrm{C}_{2} \mathrm{H}_{5} \mathrm{CN}, v_{13}=1 / v_{21}=1\end{array}$ \\
\hline 472 & $38_{6,32}-37_{6,31}$ & 235612.906 & 10 & 351 & 792.6 & 131 & $\begin{array}{l}\text { Blend with } \mathrm{CH}_{3} \mathrm{CH}_{3} \mathrm{CO},{ }^{13} \mathrm{CH}_{3} \mathrm{OH}, v_{t}=1 \text {, and } \\
\mathrm{C}_{2} \mathrm{H}_{5} \mathrm{CN}, v_{13}=1 / v_{21}=1\end{array}$ \\
\hline 473 & $38_{6,33}-37_{5,32}$ & 235699.420 & 8 & 348 & 136.0 & 131 & Blend with $\mathrm{CH}_{3} \mathrm{OCH}_{3}$, uncertain baseline \\
\hline 474 & $41_{3,38}-40_{4,37}$ & 235705.579 & 10 & 370 & 252.1 & 131 & Blend with $\mathrm{CH}_{3} \mathrm{OCH}_{3}$, uncertain baseline \\
\hline 475 & $41_{4,38}-40_{4,37}$ & 235720.329 & 10 & 370 & 860.1 & 131 & Blend with $\mathrm{CH}_{3} \mathrm{OCH}_{3}$, uncertain baseline \\
\hline 476 & $41_{3,38}-40_{3,37}$ & 235728.110 & 10 & 370 & 860.0 & 131 & Blend with $U$-line, uncertain baseline \\
\hline 477 & $67_{13,55}-66_{14,52}$ & 235732.617 & 481 & 838 & 79.3 & 131 & Blend with $U$-line, uncertain baseline \\
\hline 478 & $41_{4,38}-40_{3,37}$ & 235742.860 & 10 & 370 & 252.1 & 131 & Baseline problem, or absorption line? \\
\hline 479 & $17_{10,8}-16_{9,7^{\star}}$ & 235876.754 & 12 & 199 & 90.8 & 131 & Strong $\mathrm{CH}_{3} \mathrm{OCHO}, \mathrm{C}_{2} \mathrm{H}_{5} \mathrm{CN}, v_{13}=1 / v_{21}=1$, and ${ }^{13} \mathrm{CH}_{3} \mathrm{OH}$ \\
\hline 481 & $39_{10,30}-38_{10,29}$ & 235923.054 & 8 & 380 & 780.4 & 131 & Blend with $\mathrm{CH}_{3} \mathrm{OCHO}$ \\
\hline 482 & $39_{10,29}-38_{10,28}$ & 235988.744 & 8 & 380 & 780.5 & 131 & Strong $\mathrm{C}_{2} \mathrm{H}_{5} \mathrm{OH}$ and ${ }^{13} \mathrm{CH}_{3} \mathrm{OH}$ \\
\hline 483 & $68_{4,64}-68_{3,65}{ }^{\star}$ & 240990.966 & 671 & 784 & 134.4 & 216 & Strong $\mathrm{CH}_{3} \mathrm{OCH}_{3}, \mathrm{C}_{2} \mathrm{H}_{5}{ }^{13} \mathrm{CN}$, and $\mathrm{CH}_{3} \mathrm{CH}_{3} \mathrm{CO}$ \\
\hline 485 & $685,64-68_{4,65}{ }^{\star}$ & 240990.969 & 671 & 784 & 134.4 & 216 & Strong $\mathrm{CH}_{3} \mathrm{OCH}_{3}, \mathrm{C}_{2} \mathrm{H}_{5}{ }^{13} \mathrm{CN}$, and $\mathrm{CH}_{3} \mathrm{CH}_{3} \mathrm{CO}$ \\
\hline 487 & $40_{12,29}-39_{12,28}$ & 240992.017 & 9 & 406 & 779.7 & 216 & Strong $\mathrm{CH}_{3} \mathrm{OCH}_{3}, \mathrm{C}_{2} \mathrm{H}_{5}{ }^{13} \mathrm{CN}$, and $\mathrm{CH}_{3} \mathrm{CH}_{3} \mathrm{CO}$ \\
\hline 488 & $40_{12,28}-39_{12,27}$ & 240992.663 & 9 & 406 & 779.7 & 216 & Strong $\mathrm{CH}_{3} \mathrm{OCH}_{3}, \mathrm{C}_{2} \mathrm{H}_{5}{ }^{13} \mathrm{CN}$, and $\mathrm{CH}_{3} \mathrm{CH}_{3} \mathrm{CO}$ \\
\hline 489 & $39_{6,33}-38_{6,32}$ & 241002.693 & 11 & 363 & 812.9 & 216 & Strong $\mathrm{C}_{2} \mathrm{H}_{5}{ }^{13} \mathrm{CN}, \mathrm{CH}_{3} \mathrm{CH}_{3} \mathrm{CO}$, and $\mathrm{C}^{34} \mathrm{~S}$ \\
\hline 490 & $42_{3,39}-41_{4,38}$ & 241081.365 & 11 & 382 & 261.4 & 216 & Blend with $\mathrm{CH}_{3}{ }^{13} \mathrm{CH}_{2} \mathrm{CN}$ and $U$-line \\
\hline 491 & $42_{4,39}-41_{4,38}$ & 241090.991 & 11 & 382 & 881.3 & 216 & Blend with $\mathrm{CH}_{3}{ }^{13} \mathrm{CH}_{2} \mathrm{CN}$ and $U$-line \\
\hline 492 & $42_{3,39}-41_{3,38}$ & 241096.116 & 11 & 382 & 881.3 & 216 & Blend with $\mathrm{CH}_{3}{ }^{13} \mathrm{CH}_{2} \mathrm{CN}$ and $U$-line \\
\hline 493 & $42_{4,39}-41_{3,38}$ & 241105.742 & 11 & 382 & 261.4 & 216 & Blend with $U$-line and $\mathrm{C}_{2} \mathrm{H}_{5} \mathrm{CN}$ \\
\hline 494 & $16_{11,5}-15_{10,6}{ }^{\star}$ & 244001.794 & 12 & 201 & 97.9 & 46 & Blend with $\mathrm{C}_{2} \mathrm{H}_{5} \mathrm{OH}$ \\
\hline 496 & $41_{6,36}-40_{6,35}$ & 244037.948 & 12 & 382 & 852.7 & 46 & Strong $\mathrm{H}_{2} \mathrm{CS}$ \\
\hline 497 & $41_{5,36}-40_{5,35}$ & 244944.066 & 12 & 382 & 853.1 & 39 & Strong CS \\
\hline 498 & $45_{0,45}-44_{1,44}{ }^{\star}$ & 245041.483 & 39 & 394 & 394.8 & 39 & Blend with $\mathrm{C}_{2} \mathrm{H}_{5} \mathrm{CN}$ and $\mathrm{C}_{2} \mathrm{H}_{5}{ }^{13} \mathrm{CN}$ \\
\hline 500 & $45_{1,45}-44_{1,44} \star$ & 245041.483 & 39 & 394 & 960.6 & 39 & Blend with $\mathrm{C}_{2} \mathrm{H}_{5} \mathrm{CN}$ and $\mathrm{C}_{2} \mathrm{H}_{5}{ }^{13} \mathrm{CN}$ \\
\hline 502 & $42_{4,38}-41_{5,37}$ & 245115.653 & 12 & 388 & 222.4 & 72 & Strong $\mathrm{CH}_{2} \mathrm{NH}$ \\
\hline 503 & $42_{5,38}-41_{5,37}$ & 245270.702 & 12 & 388 & 877.3 & 72 & Strong $\mathrm{HC}_{3} \mathrm{~N}, v_{4}=1$ and $\mathrm{C}_{2} \mathrm{H}_{5}{ }^{13} \mathrm{CN}$ \\
\hline 504 & $45_{18,27}-45_{17,28^{\star}}$ & 245277.970 & 79 & 528 & 194.5 & 72 & Strong $\mathrm{C}_{2} \mathrm{H}_{5}{ }^{13} \mathrm{CN}$ \\
\hline 506 & $42_{4,38}-41_{4,37}$ & 245344.019 & 12 & 388 & 877.3 & 72 & Strong $\mathrm{SO}_{2}$ and $\mathrm{CH}_{3} \mathrm{CH}_{3} \mathrm{CO}$ \\
\hline 507 & $44_{18,26}-44_{17,27^{\star}}$ & 245407.219 & 79 & 515 & 188.3 & 72 & Blend with $\mathrm{CH}_{3} \mathrm{CH}_{3} \mathrm{CO}$ \\
\hline 509 & $21_{9,13}-20_{8,12}$ & 245470.669 & 12 & 215 & 86.9 & 72 & Strong $\mathrm{C}_{2} \mathrm{H}_{5} \mathrm{CN}, v_{13}=1 / v_{21}=1$ and $\mathrm{C}_{2} \mathrm{H}_{3} \mathrm{CN}, v_{11}=1$ \\
\hline 510 & $21_{9,12}-20_{8,13}$ & 245471.188 & 12 & 215 & 86.9 & 72 & Strong $\mathrm{C}_{2} \mathrm{H}_{5} \mathrm{CN}, v_{13}=1 / v_{21}=1$ and $\mathrm{C}_{2} \mathrm{H}_{3} \mathrm{CN}, v_{11}=1$ \\
\hline 511 & $86_{10,76}-86_{9,77}$ & 245475.958 & 1519 & 1239 & 344.4 & 72 & Strong $\mathrm{C}_{2} \mathrm{H}_{5} \mathrm{CN}, v_{13}=1 / v_{21}=1$ and $\mathrm{C}_{2} \mathrm{H}_{3} \mathrm{CN}, v_{11}=1$ \\
\hline 512 & $42_{5,38}-41_{4,37}$ & 245499.068 & 12 & 388 & 222.4 & 72 & Strong $\mathrm{HC}^{13} \mathrm{CCN}, v_{7}=1$ \\
\hline 513 & $43_{18,25}-43_{17,26^{\star}}$ & 245525.550 & 78 & 502 & 182.0 & 72 & Strong $\mathrm{HCC}^{13} \mathrm{CN}, v_{7}=1$ \\
\hline 515 & $42_{18,24}-42_{17,25}^{\star}$ & 245633.528 & 78 & 490 & 175.7 & 53 & Blend with $\mathrm{CH}_{2}{ }^{13} \mathrm{CHCN}$ and $\mathrm{C}_{2} \mathrm{H}_{3} \mathrm{CN}, v_{15}=2$ \\
\hline 517 & $77_{7,70}-77_{7,71}{ }^{\star}$ & 245718.043 & 974 & 998 & 32.3 & 53 & Blend with $\mathrm{CH}_{2}{ }^{13} \mathrm{CHCN}$ and $\mathrm{HNCO}, v_{5}=1$ \\
\hline 519 & $77_{8,70}-77_{7,71} \star$ & 245718.533 & 974 & 998 & 237.4 & 53 & Blend with $\mathrm{CH}_{2}{ }^{13} \mathrm{CHCN}$ and $\mathrm{HNCO}, v_{5}=1$ \\
\hline 521 & $41_{22,19}-40_{22,18^{\star}}$ & 245718.666 & 11 & 532 & 625.3 & 53 & Blend with $\mathrm{CH}_{2}{ }^{13} \mathrm{CHCN}$ and $\mathrm{HNCO}, v_{5}=1$ \\
\hline 523 & $41_{23,18}-40_{23,17^{\star}}$ & 245719.705 & 12 & 547 & 601.8 & 53 & Blend with $\mathrm{CH}_{2}{ }^{13} \mathrm{CHCN}$ and $\mathrm{HNCO}, v_{5}=1$ \\
\hline 525 & $41_{21,20}-40_{21,19^{\star}}^{\star}$ & 245730.112 & 12 & 517 & 647.8 & 53 & Blend with $\mathrm{HNCO}, v_{5}=1$ \\
\hline 527 & $41_{24,17}-40_{24,16^{\star}}$ & 245731.600 & 12 & 563 & 577.2 & 53 & Blend with $\mathrm{HNCO}, v_{5}=1$ \\
\hline 529 & $41_{18,23}-41_{17,24^{\star}}$ & 245731.700 & 78 & 478 & 169.5 & 53 & Blend with $\mathrm{HNCO}, v_{5}=1$ \\
\hline 531 & $42_{8,34}-42_{5,37}$ & 245748.107 & 35 & 405 & 8.6 & 53 & Strong $\mathrm{CH}_{2}{ }^{13} \mathrm{CHCN}$ and $\mathrm{CH}_{3} \mathrm{OCHO}$ \\
\hline 532 & $41_{25,16}-40_{25,15^{\star}}$ & 245753.043 & 13 & 579 & 551.6 & 53 & Strong $\mathrm{CH}_{2}{ }^{13} \mathrm{CHCN}, \mathrm{CH}_{3} \mathrm{OCHO}$, and $\mathrm{HC}_{3} \mathrm{~N}, v_{5}=1 / v_{7}=3$ \\
\hline 534 & $41_{20,21}-40_{20,20}^{\star}$ & 245756.100 & 12 & 504 & 669.2 & 53 & Strong $\mathrm{CH}_{3} \mathrm{OCHO}$ and $\mathrm{HC}_{3} \mathrm{~N}, v_{5}=1 / v_{7}=3$ \\
\hline 536 & $41_{26,15}-40_{26,14^{\star}}$ & 245782.976 & 14 & 597 & 525.1 & 53 & Strong $\mathrm{CH}_{2}{ }^{13} \mathrm{CHCN}$ and $\mathrm{C}_{2} \mathrm{H}_{5} \mathrm{CN}, v_{13}=1 / v_{21}=1$ \\
\hline 538 & $41_{19,22}-40_{19,21}{ }^{\star}$ & 245799.265 & 12 & 490 & 689.7 & 53 & Blend with $\mathrm{CH}_{2} \mathrm{CH}^{13} \mathrm{CN}$ \\
\hline 540 & $48_{8,40}-47_{9,39}$ & 245812.073 & 103 & 487 & 81.9 & 53 & Strong $\mathrm{CH}_{2} \mathrm{CH}^{13} \mathrm{CN}$ and $\mathrm{CH}_{2}{ }^{13} \mathrm{CHCN}$ \\
\hline 541 & $41_{27,14}-40_{27,13}{ }^{\star}$ & 245820.530 & 15 & 614 & 497.4 & 53 & Strong $\mathrm{CH}_{2} \mathrm{CH}^{13} \mathrm{CN}$ and $\mathrm{CH}_{2}{ }^{13} \mathrm{CHCN}$ \\
\hline 543 & $40_{18,22}-40_{17,23}{ }^{\star}$ & 245820.595 & 78 & 466 & 163.2 & 53 & Strong $\mathrm{CH}_{2} \mathrm{CH}^{13} \mathrm{CN}$ and $\mathrm{CH}_{2}{ }^{13} \mathrm{CHCN}$ \\
\hline 545 & $41_{18,23}-40_{18,22}{ }^{\star}$ & 245863.030 & 12 & 478 & 708.9 & 53 & Blend with $\mathrm{CH}_{2} \mathrm{CH}^{13} \mathrm{CN}$ \\
\hline 547 & $41_{28,13}-40_{28,12^{\star}}$ & 245864.987 & 16 & 633 & 468.6 & 53 & Blend with $\mathrm{CH}_{2} \mathrm{CH}^{13} \mathrm{CN}$ \\
\hline 549 & $39_{18,21}-39_{17,22}^{\star}$ & 245900.727 & 78 & 455 & 157.0 & 53 & Strong $\mathrm{CH}_{3} \mathrm{OCHO}$ and $\mathrm{CH}_{2} \mathrm{CH}^{13} \mathrm{CN}$ \\
\hline 551 & $41_{29,12}-40_{29,11^{\star}}^{\star}$ & 245915.751 & 17 & 652 & 438.9 & 53 & Strong $\mathrm{CH}_{2} \mathrm{CH}^{13} \mathrm{CN}$ and $\mathrm{CH}_{2}{ }^{13} \mathrm{CHCN}$ \\
\hline 553 & $26_{7,19}-25_{6,20}$ & 245927.818 & 12 & 239 & 70.3 & 53 & Blend with $\mathrm{CH}_{2} \mathrm{CH}^{13} \mathrm{CN}$ \\
\hline 554 & $41_{17,24}-40_{17,23}{ }^{\star}$ & 245951.925 & 12 & 466 & 727.2 & 53 & Blend with $\mathrm{CH}_{3} \mathrm{CH}_{3} \mathrm{CO}, v_{t}=1$ \\
\hline
\end{tabular}


A. Belloche et al.: Detection and chemical modeling of ethyl formate and $n$-propyl cyanide in Sgr B2(N), Online Material $p 32$

Table 10. continued.

\begin{tabular}{|c|c|c|c|c|c|c|c|}
\hline$N^{a}$ & $\begin{array}{c}\text { Transition }^{b} \\
\text { (2) }\end{array}$ & $\begin{array}{c}\text { Frequency } \\
(\mathrm{MHz}) \\
\text { (3) }\end{array}$ & $\begin{array}{l}\text { Unc. }^{c} \\
(\mathrm{kHz}) \\
(4)\end{array}$ & $\begin{array}{l}E_{1}^{d} \\
(\mathrm{~K}) \\
(5)\end{array}$ & $\begin{array}{l}S \mu^{2} \\
\left(\mathrm{D}^{2}\right) \\
(6)\end{array}$ & $\begin{array}{c}\sigma^{e} \\
(\mathrm{mK}) \\
(7)\end{array}$ & Comments \\
\hline 556 & $41_{30,11}-40_{30,10}{ }^{\star}$ & 245972.316 & 19 & 672 & 408.0 & 53 & Blend with $\mathrm{C}_{2} \mathrm{H}_{5} \mathrm{CN}, v_{13}=1 / v_{21}=1$ and $v=0$, and $U$-line \\
\hline 558 & $38_{18,20}-38_{17,21}$ * & 245972.593 & 78 & 443 & 150.7 & 53 & Blend with $\mathrm{C}_{2} \mathrm{H}_{5} \mathrm{CN}, v_{13}=1 / v_{21}=1$ and $v=0$, and $U$-line \\
\hline 560 & $41_{31,10}-40_{31,9^{\star}}$ & 246034.256 & 22 & 692 & 376.2 & 53 & Blend with $\mathrm{C}_{2} \mathrm{H}_{5} \mathrm{CN}, v_{13}=1 / v_{21}=1$ and $\mathrm{CH}_{3} \mathrm{OCHO}$ \\
\hline 562 & $37_{18,19}-37_{17,20}{ }^{\star}$ & 246036.674 & 78 & 432 & 144.4 & 53 & Blend with $\mathrm{C}_{2} \mathrm{H}_{5} \mathrm{CN}, v_{13}=1 / v_{21}=1$ and $\mathrm{CH}_{3} \mathrm{OCHO}$ \\
\hline 564 & $27_{7,21}-26_{6,20}$ & 247058.498 & 12 & 246 & 70.8 & 68 & Strong $\mathrm{CH}_{3} \mathrm{OCHO}$ \\
\hline 565 & $63_{12,51}-62_{13,50}$ & 247101.751 & 518 & 754 & 76.5 & 68 & Blend with $\mathrm{C}_{2} \mathrm{H}_{3} \mathrm{CN}$ and $\mathrm{HC}_{3} \mathrm{~N}, v_{7}=2$ \\
\hline 566 & $41_{12,30}-40_{12,29}$ & 247104.436 & 10 & 418 & 803.0 & 68 & Blend with $\mathrm{C}_{2} \mathrm{H}_{3} \mathrm{CN}$ and $\mathrm{HC}_{3} \mathrm{~N}, v_{7}=2$ \\
\hline 567 & $41_{12,29}-40_{12,28}$ & 247105.557 & 10 & 418 & 803.0 & 68 & Blend with $\mathrm{C}_{2} \mathrm{H}_{3} \mathrm{CN}$ and $\mathrm{HC}_{3} \mathrm{~N}, v_{7}=2$ \\
\hline 568 & $41_{6,36}-40_{5,35}$ & 247299.045 & 10 & 382 & 168.3 & 68 & Blend with $\mathrm{C}_{2} \mathrm{H}_{3} \mathrm{CN}, v_{15}=1$ \\
\hline 569 & $41_{7,35}-40_{7,34}$ & 247432.588 & 13 & 387 & 849.3 & 68 & Blend with $\mathrm{C}_{2} \mathrm{H}_{5} \mathrm{OCHO}$, $\mathrm{t}-\mathrm{HCOOH}$, and ${ }^{34} \mathrm{SO}_{2}$ \\
\hline 570 & $42_{17,25}-41_{17,24^{\star}}$ & 251968.635 & 13 & 478 & 752.3 & 42 & Blend with $\mathrm{CH}_{3} \mathrm{OCH}_{3}$ \\
\hline 572 & $27_{7,20}-26_{6,21}$ & 252059.917 & 12 & 246 & 69.6 & 42 & $\begin{array}{l}\text { Blend with } \mathrm{CH}_{3}{ }^{13} \mathrm{CH}_{2} \mathrm{CN}, \mathrm{C}_{2} \mathrm{H}_{5} \mathrm{CN}, v_{20}=1, \mathrm{a}-\mathrm{C}_{3} \mathrm{H}_{7} \mathrm{CN} \text {, and } \\
U \text {-line }\end{array}$ \\
\hline 573 & $42_{32,10}-41_{32,9^{\star}}$ & 252075.949 & 25 & 725 & 377.4 & 42 & Blend with a- $\mathrm{C}_{3} \mathrm{H}_{7} \mathrm{CN}$ and $\mathrm{t}-\mathrm{HCOOH}$ \\
\hline 575 & $15_{12,3}-14_{11,4^{\star}}$ & 252090.157 & 12 & 204 & 105.6 & 42 & Strong t- $\mathrm{HCOOH}$ and $\mathrm{CH}_{3} \mathrm{OH}$ \\
\hline 577 & $42_{16,27}-41_{16,26^{\star}}$ & 252100.361 & 12 & 467 & 769.0 & 42 & Strong $\mathrm{CH}_{3} \mathrm{OH}$ and $\mathrm{C}_{2} \mathrm{H}_{5} \mathrm{CN}, v_{13}=1 / v_{21}=1$ \\
\hline 579 & $42_{15,28}-41_{15,27^{\star}}$ & 252274.857 & 12 & 456 & 784.8 & 42 & Strong $\mathrm{C}_{2} \mathrm{H}_{5} \mathrm{CN}, v_{13}=1 / v_{21}=1$ \\
\hline 581 & $64_{1,63}-64_{0,64}^{\star}$ & 252277.352 & 860 & 678 & 33.6 & 42 & Strong $\mathrm{C}_{2} \mathrm{H}_{5} \mathrm{CN}, v_{13}=1 / v_{21}=1$ \\
\hline 583 & $64_{1,63}-64_{1,64}^{\star}$ & 252277.352 & 860 & 678 & 5.7 & 42 & Strong $\mathrm{C}_{2} \mathrm{H}_{5} \mathrm{CN}, v_{13}=1 / v_{21}=1$ \\
\hline 585 & $25_{8,18}-24_{7,17}$ & 254150.394 & 12 & 236 & 81.3 & 32 & Blend with $\mathrm{CH}_{3} \mathrm{CH}_{3} \mathrm{CO}$ and $U$-line \\
\hline 586 & $13_{13,0}-12_{12,1}^{\star}$ & 254156.954 & 13 & 204 & 114.2 & 32 & Blend with $\mathrm{CH}_{3} \mathrm{CH}_{3} \mathrm{CO}$ and $U$-line \\
\hline 588 & $25_{8,17}-24_{7,18}$ & 254324.253 & 12 & 236 & 81.3 & 32 & Strong ${ }^{13} \mathrm{CH}_{3} \mathrm{OH}$ \\
\hline 589 & $42_{9,34}-41_{9,33}$ & 255230.622 & 14 & 409 & 858.3 & 217 & Strong $\mathrm{C}_{2} \mathrm{H}_{5} \mathrm{CN}, v_{20}=1$ and $\mathrm{NH}_{2} \mathrm{CHO}$ \\
\hline 590 & $43_{5,38}-42_{5,37}$ & 255329.346 & 15 & 405 & 895.6 & 217 & Strong $\mathrm{C}_{2} \mathrm{H}_{5} \mathrm{CN}, v_{13}=1 / v_{21}=1$ and $\mathrm{HC}_{3} \mathrm{~N}, v_{7}=1$ \\
\hline 591 & $41_{7,34}-40_{7,33}$ & 255562.496 & 16 & 389 & 855.2 & 217 & Strong $\mathrm{SO}_{2}$ and $\mathrm{CH}_{2} \mathrm{CH}^{13} \mathrm{CN}$ \\
\hline 592 & $46_{7,39}-45_{8,38}$ & 257971.741 & 60 & 454 & 112.3 & 1127 & Strong t- $\mathrm{HCOOH}$ and $\mathrm{CH}_{3} \mathrm{CN}, v_{8}=1$ \\
\hline 593 & $43_{31,12}-42_{31,11}{ }^{\star}$ & 257980.769 & 18 & 716 & 442.3 & 1127 & Strong t- $\mathrm{HCOOH}$ and $\mathrm{CH}_{3} \mathrm{CN}, v_{8}=1$ \\
\hline 595 & $43_{17,26}-42_{17,25}{ }^{\star}$ & 257986.516 & 13 & 490 & 777.1 & 1127 & Strong t- $\mathrm{HCOOH}$ and $\mathrm{CH}_{3} \mathrm{CN}, v_{8}=1$ \\
\hline 597 & $55_{19,36}-55_{18,37^{\star}}$ & 258045.723 & 118 & 685 & 252.3 & 1127 & Strong $\mathrm{CH}_{3} \mathrm{CN}, v_{8}=1$ \\
\hline 599 & $43_{32,11}-42_{32,10^{\star}}^{\star}$ & 258048.624 & 22 & 737 & 410.9 & 1127 & Strong $\mathrm{CH}_{3} \mathrm{CN}, v_{8}=1$ \\
\hline 601 & 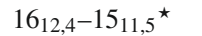 & 258080.161 & 12 & 208 & 106.2 & 1127 & Strong $\mathrm{CH}_{3} \mathrm{OCHO}, \mathrm{CH}_{3}{ }^{13} \mathrm{CN}, v_{8}=1$, and $\mathrm{NH}_{2} \mathrm{CN}$ \\
\hline 603 & $43_{33,10}-42_{33,9^{\star}}$ & 258121.617 & 31 & 759 & 378.5 & 1127 & Strong $\mathrm{CH}_{3} \mathrm{CN}, v_{8}=1$ and $\mathrm{CH}_{3} \mathrm{OCHO}$ \\
\hline 605 & $43_{16,28}-42_{16,27^{\star}}$ & 258130.483 & 13 & 479 & 793.5 & 1127 & Strong $\mathrm{CH}_{3} \mathrm{OCHO}$ and $\mathrm{CH}_{3} \mathrm{CN}, v_{8}=1$ \\
\hline 607 & $43_{15,29}-42_{15,28}{ }^{\star}$ & 258320.451 & 13 & 469 & 809.0 & 1127 & Strong $\mathrm{CH}_{3} \mathrm{CN}, v_{8}=1$ \\
\hline 609 & $43_{13,31}-42_{13,30}$ & 258903.344 & 12 & 450 & 836.8 & 1609 & Noisy, blend with ${ }^{13} \mathrm{CH}_{2} \mathrm{CHCN}$ \\
\hline 610 & $43_{13,30}-42_{13,29}$ & 258903.558 & 12 & 450 & 836.8 & 1609 & Noisy, blend with ${ }^{13} \mathrm{CH}_{2} \mathrm{CHCN}$ \\
\hline 611 & $43_{11,32}$ & 260000.636 & 13 & 434 & 860.9 & 413 & Strong $\mathrm{CH}_{3} \mathrm{OCH}_{3}$ and $\mathrm{CH}_{2}{ }^{13} \mathrm{CHCN}$ \\
\hline 612 & $40_{19,21}-40_{18,22}{ }^{\star}$ & 260004.028 & 112 & 478 & 158.0 & 413 & Strong $\mathrm{CH}_{3} \mathrm{OCH}_{3}$ and $\mathrm{CH}_{2}{ }^{13} \mathrm{CHCN}$ \\
\hline 614 & $26_{8,18}-25_{7,19}$ & 260060.358 & 12 & 243 & 81.7 & 413 & Strong $\mathrm{C}_{2} \mathrm{H}_{5} \mathrm{CN}, v_{20}=1$ and $\mathrm{CH}_{3} \mathrm{OH}$ \\
\hline 615 & $39_{19,20}-39_{18,21}$ * & 260060.729 & 112 & 466 & 151.7 & 413 & Strong $\mathrm{C}_{2} \mathrm{H}_{5} \mathrm{CN}, v_{20}=1$ and $\mathrm{CH}_{3} \mathrm{OH}$ \\
\hline 617 & $38_{19,19}-38_{18,20}{ }^{\star}$ & 260110.774 & 112 & 455 & 145.4 & 413 & Strong $\mathrm{C}_{2} \mathrm{H}_{5} \mathrm{OH}$ and $\mathrm{C}_{2} \mathrm{H}_{5} \mathrm{CN}, v_{20}=1$ \\
\hline 619 & $14_{13,1}-13_{12,2^{\star}}$ & 260151.979 & 13 & 207 & 114.1 & 413 & Strong $\mathrm{C}_{2} \mathrm{H}_{5} \mathrm{CN}, v_{20}=1$ and $\mathrm{C}_{2} \mathrm{H}_{5} \mathrm{CN}$ \\
\hline 621 & $37_{19,18}-37_{18,19^{\star}}$ & 260154.562 & 113 & 444 & 139.0 & 413 & Strong $\mathrm{C}_{2} \mathrm{H}_{5} \mathrm{CN}, v_{20}=1$ and $\mathrm{C}_{2} \mathrm{H}_{5} \mathrm{CN}$ \\
\hline 623 & $36_{19,17}-36_{18,18^{\star}}$ & 260192.478 & 113 & 434 & 132.7 & 413 & Strong $\mathrm{NH}_{2} \mathrm{CHO}$ and $\mathrm{CH}_{2} \mathrm{CO}$ \\
\hline 625 & $44_{6,39}-43_{6,38}$ & 260216.245 & 17 & 418 & 916.5 & 413 & Strong $\mathrm{C}_{2} \mathrm{H}_{5} \mathrm{CN}$ and $\mathrm{C}_{2} \mathrm{H}_{5} \mathrm{CN}, v_{20}=1$ \\
\hline 626 & $35_{19,16}-35_{18,17^{\star}}$ & 260224.898 & 113 & 423 & 126.2 & 413 & Strong $\mathrm{C}_{2} \mathrm{H}_{5} \mathrm{CN}, v_{20}=1$ and $\mathrm{C}_{2} \mathrm{H}_{5} \mathrm{CN}$ \\
\hline 628 & $34_{19,15}-34_{18,16^{\star}}$ & 260252.187 & 113 & 413 & 119.8 & 413 & $\begin{array}{l}\text { Strong } \mathrm{C}_{2} \mathrm{H}_{5} \mathrm{OH}, \mathrm{CH}_{3} \mathrm{OCHO}, \mathrm{H}^{13} \mathrm{CO}^{+} \text {, and } \\
\mathrm{C}_{2} \mathrm{H}_{5} \mathrm{CN}, v_{13}=1 / v_{21}=1\end{array}$ \\
\hline 630 & $73_{14,60}-72_{15,57}$ & 260255.958 & 771 & 968 & 86.0 & 413 & $\begin{array}{l}\text { Strong } \mathrm{C}_{2} \mathrm{H}_{5} \mathrm{OH}, \mathrm{CH}_{3} \mathrm{OCHO}, \mathrm{H}^{13} \mathrm{CO}^{+} \text {, and } \\
\mathrm{C}_{2} \mathrm{H}_{5} \mathrm{CN}, v_{13}=1 / v_{21}=1\end{array}$ \\
\hline 631 & $19_{19,0}-19_{18,1}{ }^{\star}$ & 260262.738 & 117 & 297 & 9.3 & 413 & Strong $\mathrm{H}^{13} \mathrm{CO}^{+}$and $\mathrm{C}_{2} \mathrm{H}_{5} \mathrm{CN}, v_{13}=1 / v_{21}=1$ \\
\hline 633 & $33_{19,14}-33_{18,15^{\star}}$ & 260274.699 & 113 & 403 & 113.3 & 413 & Strong $\mathrm{C}_{2} \mathrm{H}_{5} \mathrm{CN}, v_{13}=1 / v_{21}=1, \mathrm{C}_{2} \mathrm{H}_{5} \mathrm{CN}, v_{20}=1$, and $\mathrm{H}_{2}{ }^{13} \mathrm{CS}$ \\
\hline 635 & $20_{19,1}-20_{18,2^{\star}}$ & 260275.506 & 117 & 302 & 18.2 & 413 & Strong $\mathrm{C}_{2} \mathrm{H}_{5} \mathrm{CN}, v_{13}=1 / v_{21}=1, \mathrm{C}_{2} \mathrm{H}_{5} \mathrm{CN}, v_{20}=1$, and $\mathrm{H}_{2}{ }^{13} \mathrm{CS}$ \\
\hline 637 & $21_{19,2}-21_{18,3^{\star}}$ & 260287.415 & 116 & 309 & 26.7 & 413 & Strong $\mathrm{H}_{2}{ }^{13} \mathrm{CS}$ and $\mathrm{C}_{2} \mathrm{H}_{5} \mathrm{CN}, v_{13}=1 / v_{21}=1$ \\
\hline 639 & $3219,13-32,18,14^{\star}$ & 260292.777 & 113 & 394 & 106.7 & 413 & Strong $\mathrm{H}_{2}{ }^{13} \mathrm{CS}$ and $\mathrm{C}_{2} \mathrm{H}_{5} \mathrm{CN}, v_{13}=1 / v_{21}=1$ \\
\hline 641 & $22_{19,3}-22_{18,4}{ }^{\star}$ & 260298.247 & 116 & 315 & 34.9 & 413 & Strong $\mathrm{H}_{2}{ }^{13} \mathrm{CS}$ and $\mathrm{C}_{2} \mathrm{H}_{5} \mathrm{CN}, v_{13}=1 / v_{21}=1$ \\
\hline 643 & $31_{19,12}-31_{18,13}{ }^{\star}$ & 260306.755 & 113 & 385 & 100.0 & 413 & Strong $\mathrm{C}_{2} \mathrm{H}_{5} \mathrm{CN}, v_{13}=1 / v_{21}=1$ \\
\hline 645 & $23_{19,4}-23_{18,5}{ }^{\star}$ & 260307.776 & 116 & 321 & 42.8 & 413 & Strong $\mathrm{C}_{2} \mathrm{H}_{5} \mathrm{CN}, v_{13}=1 / v_{21}=1$ \\
\hline 647 & $24_{19,5}-24_{18,6}^{\star}$ & 260315.766 & 115 & 328 & 50.5 & 413 & Strong $\mathrm{C}_{2} \mathrm{H}_{5} \mathrm{CN}, v_{13}=1 / v_{21}=1$ \\
\hline 649 & $30_{19,11}-30_{18,12^{\star}}$ & 260316.958 & 114 & 376 & 93.3 & 413 & Strong $\mathrm{C}_{2} \mathrm{H}_{5} \mathrm{CN}, v_{13}=1 / v_{21}=1, \mathrm{CH}_{3} \mathrm{OCH}_{3}$, and $\mathrm{C}_{2} \mathrm{H}_{5} \mathrm{CN}$ \\
\hline 651 & $25_{19,6}-25_{18,7^{\star}}^{\star}$ & 260321.972 & 115 & 336 & 58.0 & 413 & Strong $\mathrm{C}_{2} \mathrm{H}_{5} \mathrm{CN}, v_{13}=1 / v_{21}=1, \mathrm{CH}_{3} \mathrm{OCH}_{3}$, and $\mathrm{C}_{2} \mathrm{H}_{5} \mathrm{CN}$ \\
\hline 653 & $29_{19,10}-29_{18,11^{\star}}$ & 260323.698 & 114 & 367 & 86.5 & 413 & Strong $\mathrm{C}_{2} \mathrm{H}_{5} \mathrm{CN}, v_{13}=1 / v_{21}=1, \mathrm{CH}_{3} \mathrm{OCH}_{3}$, and $\mathrm{C}_{2} \mathrm{H}_{5} \mathrm{CN}$ \\
\hline 655 & $26_{19,7}-26_{18,8}{ }^{\star}$ & 260326.138 & 115 & 343 & 65.4 & 413 & Strong $\mathrm{CH}_{3} \mathrm{OCH}_{3}$ and $\mathrm{C}_{2} \mathrm{H}_{5} \mathrm{CN}$ \\
\hline
\end{tabular}


A. Belloche et al.: Detection and chemical modeling of ethyl formate and $n$-propyl cyanide in Sgr B2(N), Online Material $p 33$

Table 10. continued.

\begin{tabular}{|c|c|c|c|c|c|c|c|}
\hline$N^{a}$ & Transition $^{b}$ & $\begin{array}{c}\text { Frequency } \\
\text { (MHz) } \\
\text { (3) }\end{array}$ & $\begin{array}{l}\text { Unc. }^{c} \\
(\mathrm{kHz}) \\
(4)\end{array}$ & $\begin{array}{l}E_{1}^{d} \\
(\mathrm{~K}) \\
(5)\end{array}$ & $\begin{array}{l}S \mu^{2} \\
\left(\mathrm{D}^{2}\right) \\
(6)\end{array}$ & $\begin{array}{l}\sigma^{e} \\
(\mathrm{mK}) \\
(7)\end{array}$ & Comments \\
\hline 657 & $28_{19,9}-28_{18,10^{\star}}$ & 260327.281 & 114 & 359 & 79.6 & 413 & Strong $\mathrm{CH}_{3} \mathrm{OCH}_{3}$ and $\mathrm{C}_{2} \mathrm{H}_{5} \mathrm{CN}$ \\
\hline 659 & $27_{19,8}-27_{18,9^{\star}}$ & 260327.999 & 114 & 351 & 72.5 & 413 & Strong $\mathrm{CH}_{3} \mathrm{OCH}_{3}$ and $\mathrm{C}_{2} \mathrm{H}_{5} \mathrm{CN}$ \\
\hline 661 & $43_{9,34}-42_{9,33}$ & 263500.082 & 18 & 422 & 881.1 & 74 & Blend with $\mathrm{C}_{2} \mathrm{H}_{5}{ }^{13} \mathrm{CN}$ and $\mathrm{CH}_{3} \mathrm{OCH}_{3}$ \\
\hline 662 & $37_{7,31}-36_{6,30}$ & 263656.862 & 14 & 340 & 85.0 & 108 & Strong $\mathrm{CH}_{3} \mathrm{OCH}_{3}$ \\
\hline 663 & $44_{23,21}-43_{23,20}$ ネ & 263662.413 & 12 & 583 & 684.9 & 108 & Strong $\mathrm{CH}_{3} \mathrm{OCH}_{3}, \mathrm{HNCO}$, and $\mathrm{HC}^{13} \mathrm{CCN}, v_{7}=1$ \\
\hline 665 & $44_{24,20}-43_{24,19}{ }^{\star}$ & 263666.216 & 12 & 599 & 662.1 & 108 & Strong $\mathrm{CH}_{3} \mathrm{OCH}_{3}, \mathrm{HNCO}$, and $\mathrm{HC}^{13} \mathrm{CCN}, v_{7}=1$ \\
\hline 667 & $44_{22,22}-43_{22,21}^{\star}$ & 263671.555 & 13 & 568 & 706.8 & 108 & Strong $\mathrm{CH}_{3} \mathrm{OCH}_{3}, \mathrm{HNCO}$, and $\mathrm{HC}^{13} \mathrm{CCN}, v_{7}=1$ \\
\hline 669 & $44_{25,19}-43_{25,18}{ }^{\star}$ & 263681.350 & 13 & 616 & 638.2 & 108 & Strong $\mathrm{HC}^{13} \mathrm{CCN}, v_{7}=1, \mathrm{HNCO}$, and $\mathrm{HN}^{13} \mathrm{CO}$ \\
\hline 671 & $44_{21,23}-43_{21,22}{ }^{\star}$ & 263695.661 & 13 & 554 & 727.7 & 108 & Strong $\mathrm{HCC}^{13} \mathrm{CN}, v_{7}=1$ and $\mathrm{C}_{2} \mathrm{H}_{5} \mathrm{OH}$ \\
\hline 673 & $44_{26,18}-43_{26,17}^{\star}$ & 263706.506 & 13 & 633 & 613.4 & 108 & Strong $\mathrm{HCC}^{13} \mathrm{CN}, v_{7}=1$ \\
\hline 675 & $44_{20,24}-43_{20,23}{ }^{\star}$ & 263737.291 & 14 & 540 & 747.7 & 108 & Strong $\mathrm{HNCO}$ \\
\hline 677 & $44_{27,17}-43_{27,16}{ }^{\star}$ & 263740.615 & 14 & 651 & 587.6 & 108 & Strong HNCO \\
\hline 679 & $44_{28,16}-43_{28,15^{\star}}$ & 263782.797 & 15 & 669 & 560.8 & 108 & Strong $\mathrm{HC}_{3} \mathrm{~N}$ and $\mathrm{CH}_{3} \mathrm{OH}, v_{t}=1$ \\
\hline 681 & $44_{19,25}-43_{19,24}{ }^{\star}$ & 263799.727 & 14 & 527 & 766.8 & 108 & Strong $\mathrm{HC}_{3} \mathrm{~N}, \mathrm{CH}_{3} \mathrm{OH}, v_{t}=1$, and $\mathrm{HC}_{3} \mathrm{~N}, v_{5}=1 / v_{7}=3$ \\
\hline 683 & $44_{29,15}-43_{29,14}$ 太 & 263832.314 & 16 & 688 & 533.0 & 108 & Blend with $\mathrm{NH}_{2} \mathrm{CHO}$ and $U$-line? \\
\hline 685 & $38_{7,32}-37_{6,31}$ & 263847.676 & 14 & 351 & 91.4 & 108 & Blend with $\mathrm{NH}_{2} \mathrm{CHO}$ \\
\hline 686 & $47_{2,45}-46_{3,44^{\star}}$ & 263860.484 & 28 & 434 & 344.1 & 108 & Blend with $\mathrm{C}_{2} \mathrm{H}_{5} \mathrm{OH}$ and $\mathrm{C}_{2} \mathrm{H}_{5}{ }^{13} \mathrm{CN}$ \\
\hline 688 & $47_{2,45}-46_{2,44}{ }^{\star}$ & 263860.546 & 28 & 434 & 992.8 & 108 & Blend with $\mathrm{C}_{2} \mathrm{H}_{5} \mathrm{OH}$ and $\mathrm{C}_{2} \mathrm{H}_{5}{ }^{13} \mathrm{CN}$ \\
\hline 690 & $44_{18,26}-43_{18,25}^{\star}$ & 263887.257 & 14 & 514 & 784.7 & 108 & Strong $\mathrm{CH}_{3} \mathrm{CH}_{3} \mathrm{CO}$ and $\mathrm{HNCO}, v_{4}=1$ \\
\hline 692 & $44_{30,14}-43_{30,13}{ }^{\star}$ & 263888.552 & 16 & 708 & 504.4 & 108 & Strong $\mathrm{CH}_{3} \mathrm{CH}_{3} \mathrm{CO}$ and $\mathrm{HNCO}, v_{4}=1$ \\
\hline 694 & $44_{17,27}-43_{17,26^{\star}}$ & 264005.588 & 14 & 502 & 801.8 & 108 & Strong $\mathrm{CH}_{3}{ }^{13} \mathrm{CH}_{2} \mathrm{CN}$ \\
\hline 696 & $17_{12,5}-16_{11,6}{ }^{\star}$ & 264068.075 & 12 & 213 & 106.9 & 108 & Blend with $\mathrm{CH}_{3} \mathrm{CH}_{3} \mathrm{CO}, v_{t}=1$ \\
\hline 698 & $46_{4,42}-45_{5,41}$ & 266675.648 & 20 & 436 & 260.6 & 91 & Strong $\mathrm{O}^{13} \mathrm{CS}$ and $\mathrm{C}_{2} \mathrm{H}_{5}{ }^{13} \mathrm{CN}$ \\
\hline 699 & $46_{5,42}-45_{5,41}$ & 266707.175 & 20 & 436 & 962.7 & 91 & Strong $\mathrm{CH}_{3} \mathrm{OH}$ \\
\hline 700 & $29_{6,23}-28_{5,24}$ & 266722.756 & 11 & 258 & 39.0 & 91 & Blend with $\mathrm{C}_{2} \mathrm{H}_{5} \mathrm{CN}, v_{13}=1 / v_{21}=1, \mathrm{CH}_{3} \mathrm{CH}_{3} \mathrm{CO}, v_{t}=1$, and $U$-line \\
\hline 701 & $46_{4,42}-45_{4,41}$ & 266722.874 & 20 & 436 & 962.6 & 91 & Blend with $\mathrm{C}_{2} \mathrm{H}_{5} \mathrm{CN}, v_{13}=1 / v_{21}=1, \mathrm{CH}_{3} \mathrm{CH}_{3} \mathrm{CO}, v_{t}=1$, and $U$-line \\
\hline 702 & $46_{5,42}-45_{4,41}$ & 266754.401 & 20 & 436 & 260.6 & 91 & Blend with $U$-line \\
\hline 703 & $44_{8,37}-43_{8,36}$ & 266788.303 & 20 & 429 & 909.3 & 91 & Blend with $U$-line \\
\hline 704 & $44_{10,35}-43_{10,34}$ & 266958.528 & 17 & 440 & 893.9 & 91 & Blend with $\mathrm{C}_{2} \mathrm{H}_{5} \mathrm{OH}$ and $U$-line \\
\hline 705 & $44_{10,34}-44_{7,37}$ & 267152.855 & 100 & 440 & 8.9 & 91 & Strong $\mathrm{NH}_{2}{ }^{13} \mathrm{CHO}, \mathrm{CH}_{3} \mathrm{CH}_{3} \mathrm{CO}$, and $\mathrm{C}_{2} \mathrm{H}_{5} \mathrm{CN}, v_{13}=1 / v_{21}=1$ \\
\hline 706 & $43_{7,36}-427,35$ & 267157.130 & 20 & 414 & 897.1 & 91 & Strong $\mathrm{NH}_{2}{ }^{13} \mathrm{CHO}, \mathrm{CH}_{3} \mathrm{CH}_{3} \mathrm{CO}$, and $\mathrm{C}_{2} \mathrm{H}_{5} \mathrm{CN}, v_{13}=1 / v_{21}=1$ \\
\hline
\end{tabular}

Notes: ${ }^{a}$ Numbering of the observed transitions associated with a modeled line stronger than $20 \mathrm{mK} .{ }^{b}$ Transitions marked with a ${ }^{\star}$ are double with a frequency difference less than $0.1 \mathrm{MHz}$. The quantum numbers of the second one are not shown. ${ }^{c}$ Frequency uncertainty. ${ }^{d}$ Lower energy level in temperature units $\left(E_{\mathrm{l}} / k_{\mathrm{B}}\right) .{ }^{e}$ Calculated rms noise level in $T_{\mathrm{mb}}$ scale. 
A. Belloche et al.: Detection and chemical modeling of ethyl formate and $n$-propyl cyanide in Sgr B2(N), Online Material $p 34$
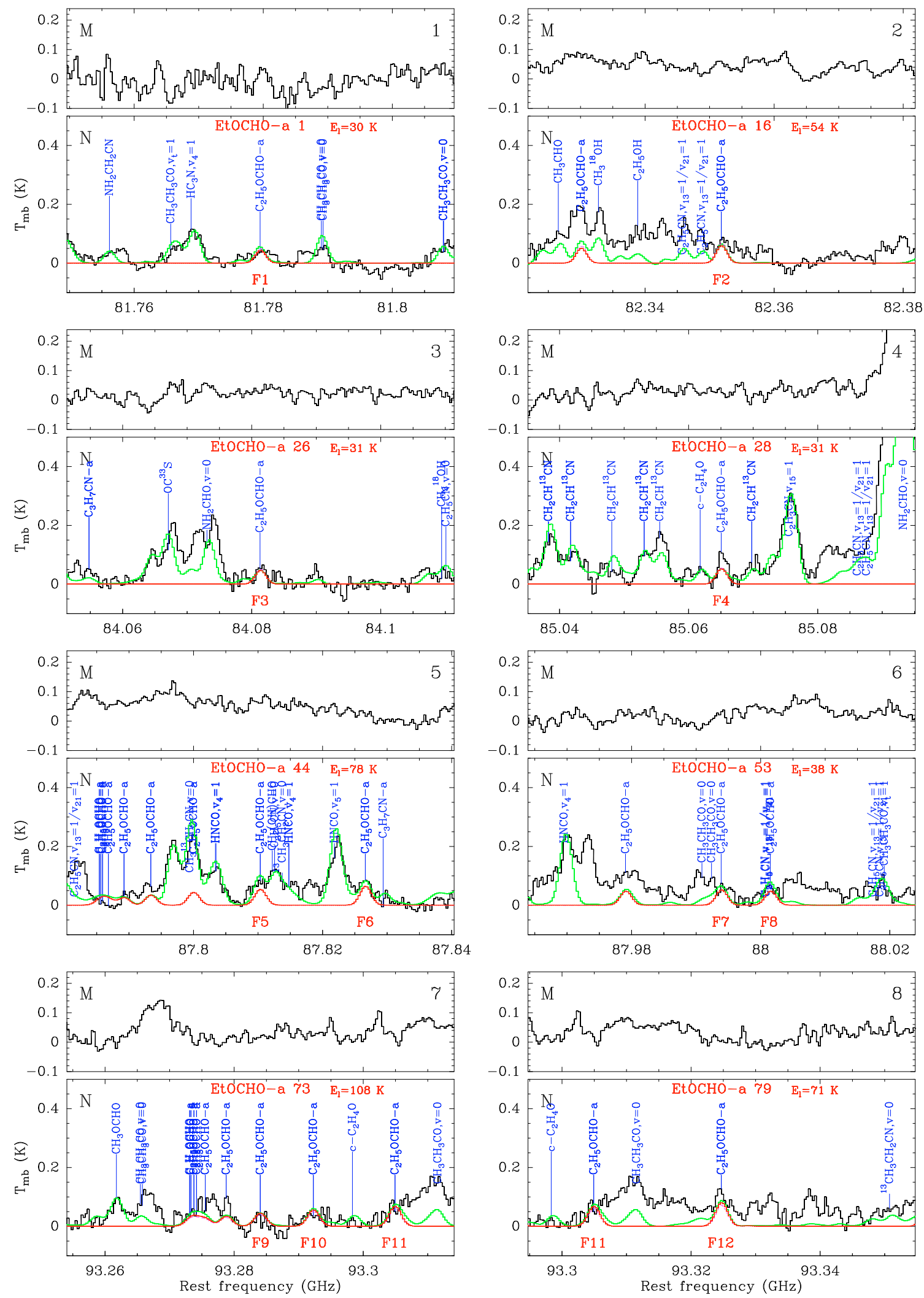

Fig. 1. Transitions of the anti-conformer of ethyl formate (EtOCHO-a) detected with the IRAM $30 \mathrm{~m}$ telescope. Each panel consists of two plots and is labeled in black in the upper right corner. The lower plot shows in black the spectrum obtained toward Sgr B2(N) in main-beam brightness temperature scale (K), while the upper plot shows the spectrum toward Sgr B2(M). The rest frequency axis is labeled in GHz. The systemic velocities assumed for Sgr B2(N) and (M) are 64 and $62 \mathrm{~km} \mathrm{~s}^{-1}$, respectively. The lines identified in the Sgr B2(N) spectrum are labeled in blue. The top red label indicates the EtOCHO-a transition centered in each plot (numbered like in Col. 1 of Table 3 ), along with the energy of its lower level in $\mathrm{K}\left(E_{\mathrm{l}} / k_{\mathrm{B}}\right)$. The other EtOCHO-a lines are labeled in blue only. The bottom red label is the feature number (see Col. 8 of Table 3 ). The green spectrum shows our LTE model containing all identified molecules, including EtOCHO-a. The LTE synthetic spectrum of EtOCHO-a alone is overlaid in red, and its opacity in dashed violet. All observed lines which have no counterpart in the green spectrum are still unidentified in Sgr B2(N). 
A. Belloche et al.: Detection and chemical modeling of ethyl formate and $n$-propyl cyanide in Sgr B2(N), Online Material $p 35$
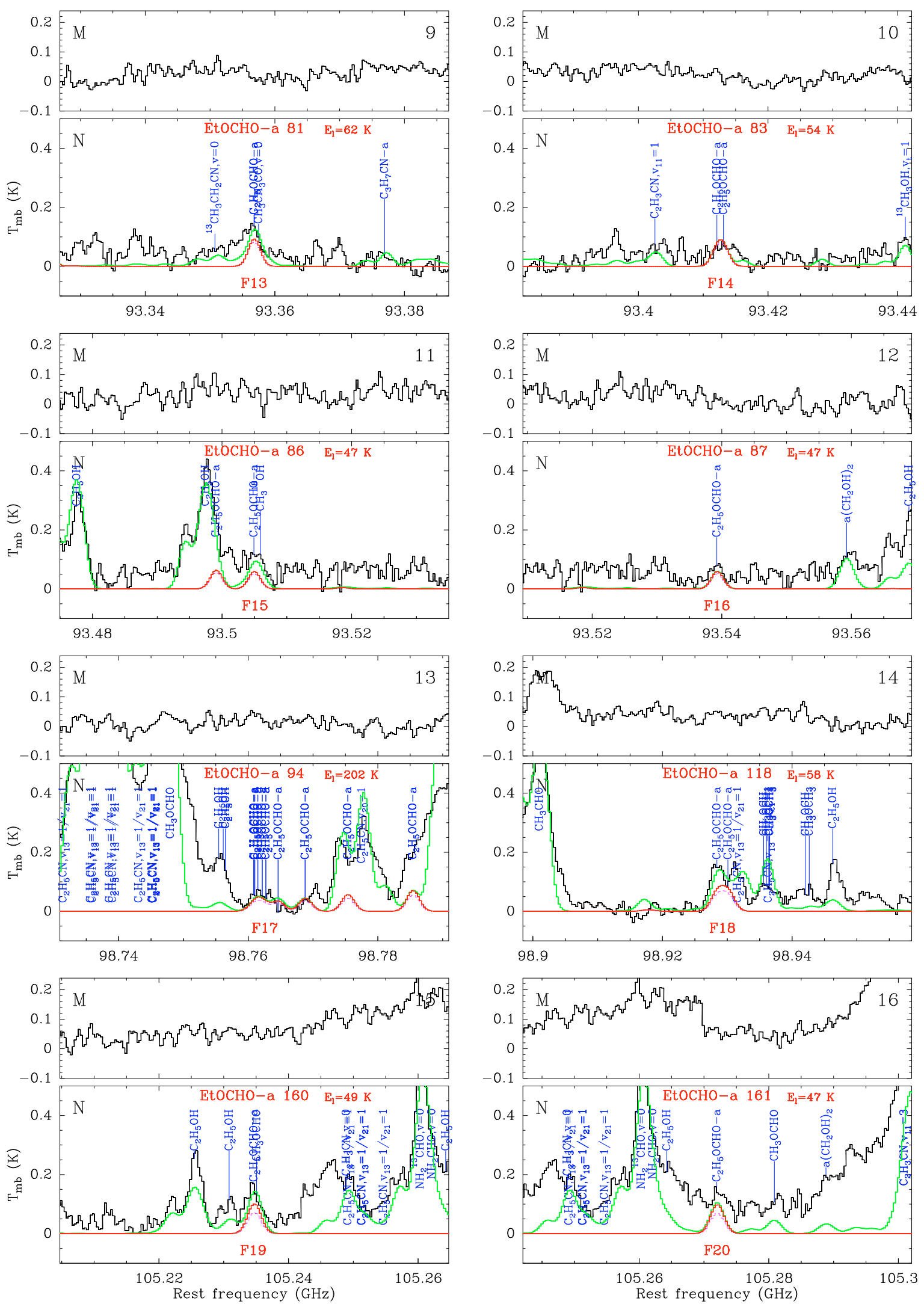

Fig. 1. continued. 
A. Belloche et al.: Detection and chemical modeling of ethyl formate and $n$-propyl cyanide in Sgr B2(N), Online Material $p 36$
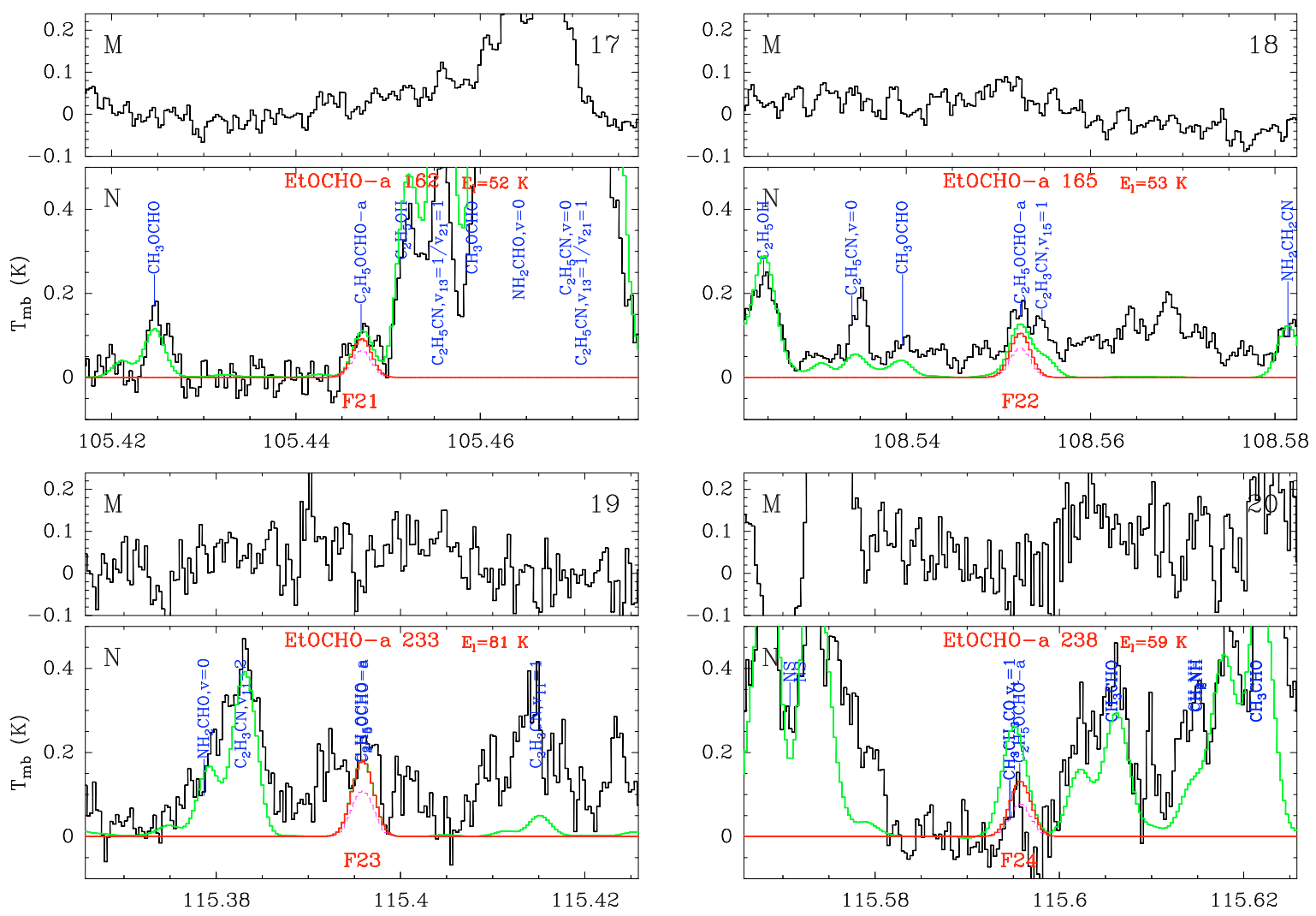

Fig. 1. continued. 
A. Belloche et al.: Detection and chemical modeling of ethyl formate and $n$-propyl cyanide in Sgr B2(N), Online Material $p 37$
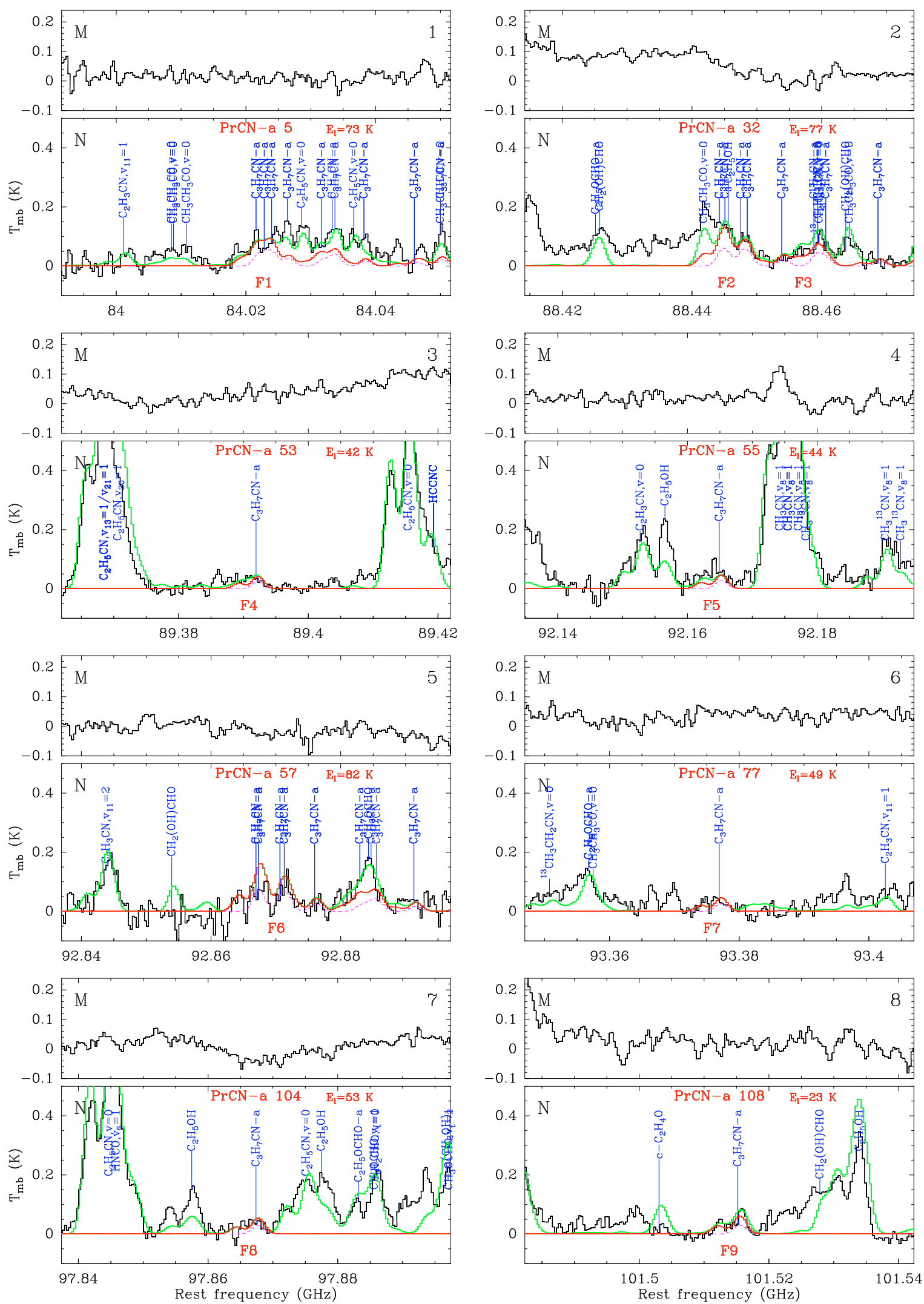

Fig. 3. Transitions of the anti-conformer of $n$-propyl cyanide (PrCN-a) detected with the IRAM $30 \mathrm{~m}$ telescope. Each panel consists of two plots and is labeled in black in the upper right corner. The lower plot shows in black the spectrum obtained toward Sgr B2(N) in main-beam brightness temperature scale (K), while the upper plot shows the spectrum toward Sgr B2(M). The rest frequency axis is labeled in GHz. The systemic velocities assumed for Sgr B2(N) and (M) are 64 and $62 \mathrm{~km} \mathrm{~s}^{-1}$, respectively. The lines identified in the Sgr B2(N) spectrum are labeled in blue. The top red label indicates the PrCN-a transition centered in each plot (numbered like in Col. 1 of Table 11), along with the energy of its lower level in $\mathrm{K}\left(E_{1} / k_{\mathrm{B}}\right)$. The other PrCN-a lines are labeled in blue only. The bottom red label is the feature number (see Col. 8 of Table 11). The green spectrum shows our LTE model containing all identified molecules, including PrCN-a. The LTE synthetic spectrum of PrCN-a alone is overlaid in red, and its opacity in dashed violet. All observed lines which have no counterpart in the green spectrum are still unidentified in Sgr B2(N). 
A. Belloche et al.: Detection and chemical modeling of ethyl formate and $n$-propyl cyanide in Sgr B2(N), Online Material $p 38$

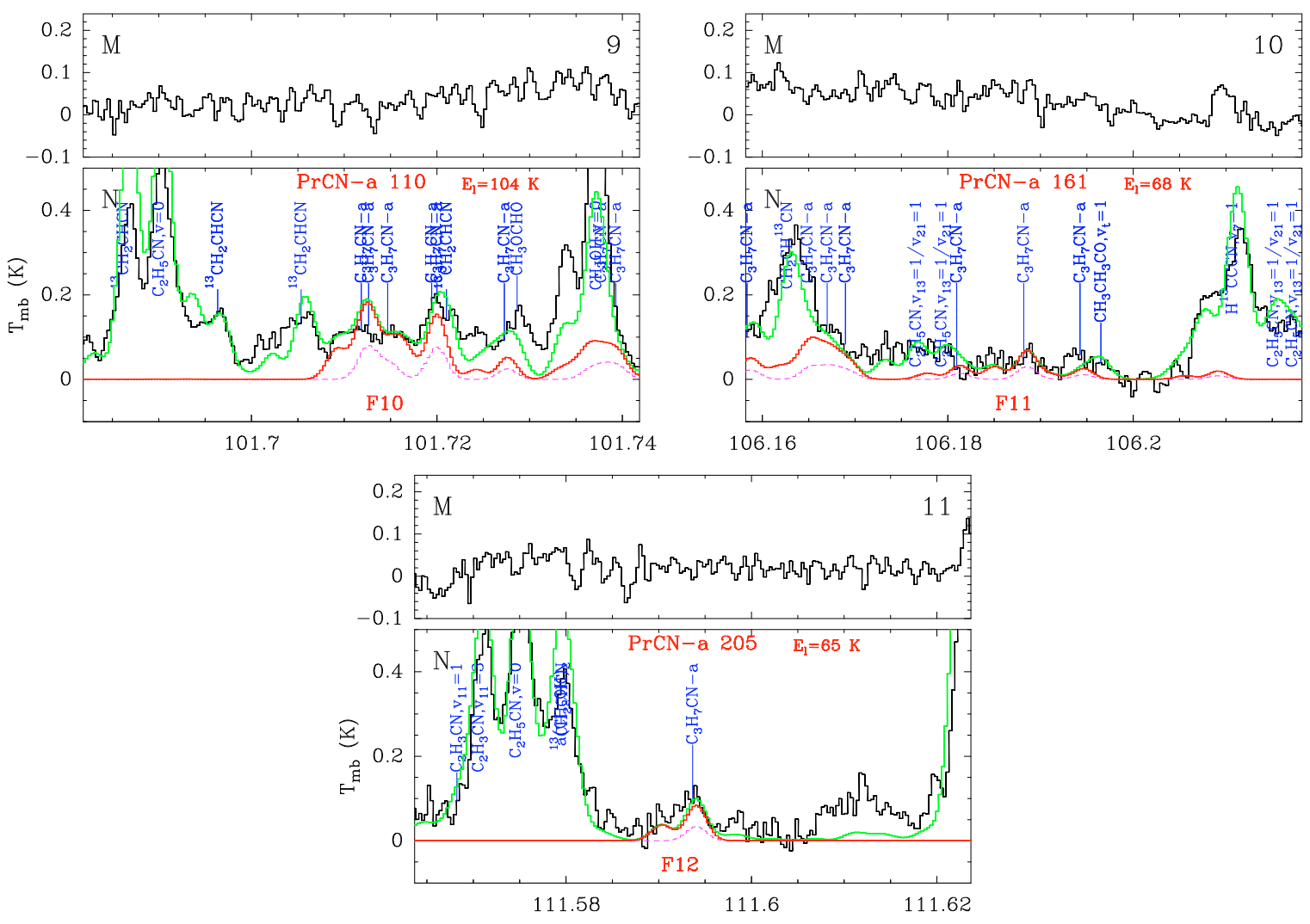

Fig. 3. continued. 
A. Belloche et al.: Detection and chemical modeling of ethyl formate and $n$-propyl cyanide in Sgr B2(N), Online Material $p 39$

\section{Appendix A: a-type and b-type lines of methyl formate}

Both $A$ and $E$ symmetry species of methyl formate $\left(\mathrm{CH}_{3} \mathrm{OCHO}\right)$ are easily detected in our spectral survey of Sgr B2(N) at $3 \mathrm{~mm}$. Sixty four lines of the $A$ species are detected in the form of 57 features in our $3 \mathrm{~mm}$ survey and 48 lines of the $E$ species in the form of 43 features. We followed the same procedure as described in Sect. 3.2 for ethyl formate to compute the population diagrams shown in Fig. A.1. In these diagrams, the $a$-type lines of methyl formate (with $\Delta K_{a}=0$ [2] and $\Delta K_{c}=1$ [2]) are marked with an additional circle. As mentioned in Sect. 3.4, both $a$ - and $b$-type lines are well fitted with the same physical model (see Table 5). Although many $a$-type transitions with $E_{\mathrm{u}} / k_{\mathrm{B}}<50 \mathrm{~K}$ look systematically too low in the population diagrams after removal of the contribution of contaminating lines (Figs. A.1b and d), this can be explained by the limitations of our radiative transfer modeling: these $a$-type transitions (of the $A$ or $E$ species) have optical depths on the order of unity, as indicated by the significant shift between the red and green crosses in the lower energy range, and overlap with $a$-type lines of the other symmetry species ( $E$ or $A$, respectively) that have significant optical depths too. Since our current complete model treats the two symmetry species as independent and our radiative transfer program computes the contributions of overlapping transitions of different species independently, the sum of the overlapping $A$ and $E$ transitions with significant optical depths is systematically overestimated. For a transition of, e.g., the $A$ species, the "contamination" by the $E$ species is overestimated and its removal in Fig. A.1b yields an underestimated residual flux. Our model could be improved by treating both symmetry species as a single molecule but this would not significantly change the physical parameters found for methyl formate and is beyond the scope of this article focused on ethyl formate and $n$-propyl cyanide. 
A. Belloche et al.: Detection and chemical modeling of ethyl formate and $n$-propyl cyanide in Sgr B2(N), Online Material $p 40$
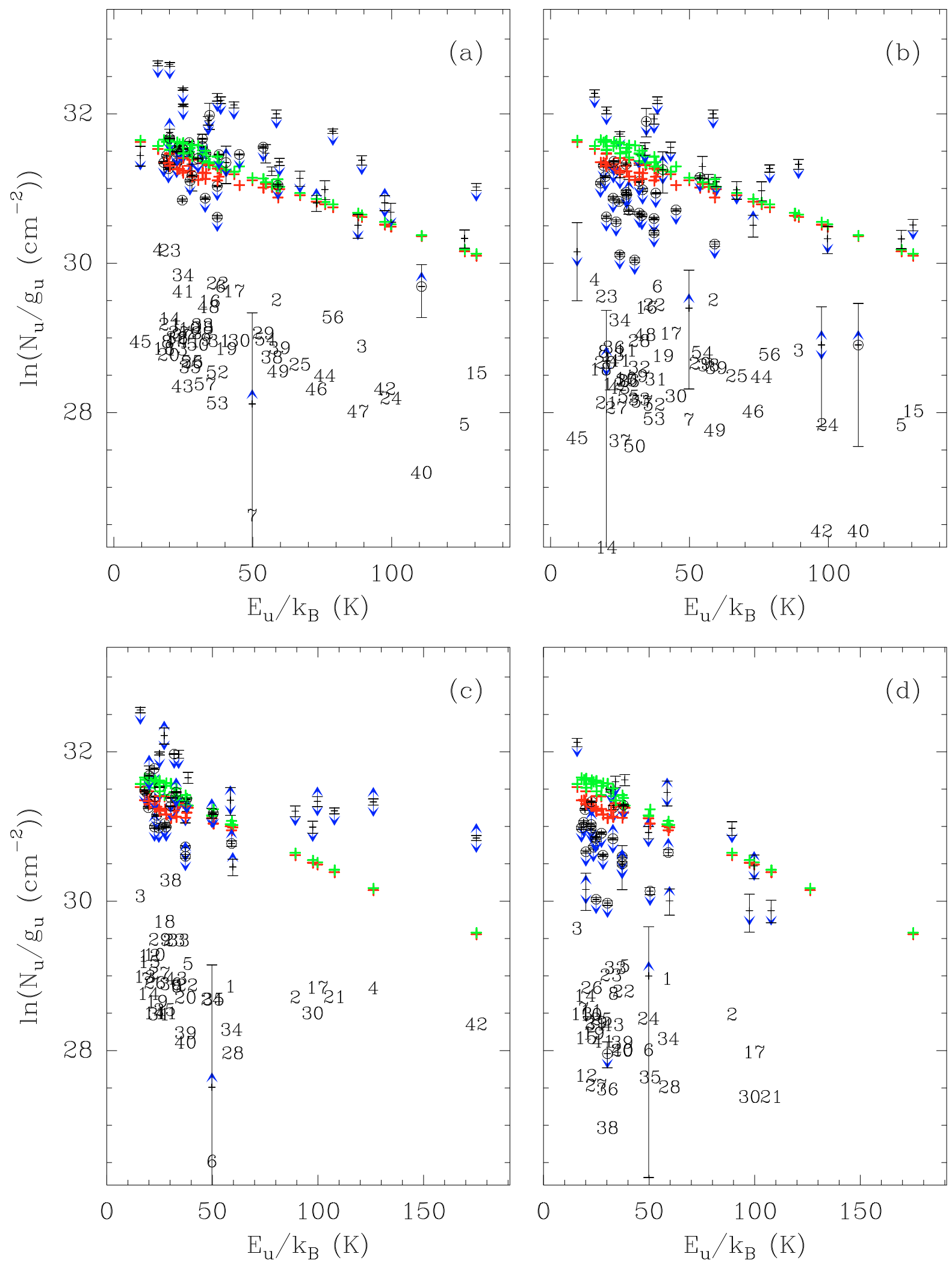

Fig. A.1. Population diagrams of the $A$ and $E$ symmetry species of methyl formate presented in the same way as for ethyl formate in Fig. 2 (see the caption of that figure for details). The $a$-type lines are marked with a circle. Panels a) and c) show the population diagrams derived from the measured integrated intensities for the $A$ and $E$ species, respectively, while panels $\mathbf{b}$ ) and d) present the respective population diagrams after removing the expected contribution from contaminating molecules. Features 4 and 42 with $E_{\mathrm{u}} / k_{\mathrm{B}}>120 \mathrm{~K}$ (see panel c)) are missing in panel d) because the removal of the contaminating lines yields negative residuals. This is due to the uncertain level of the baseline that looks overestimated for both features in the observed spectrum. 